\title{
Differential Sensitivity to Toxicity of Statins: A Comparison of Liver and Muscle Cell Lines
}

by

\section{Rowena Gee}

Submitted for the degree of Doctor of Philosophy

Department of Biochemistry and Physiology

Faculty of Health and Medical Sciences

University of Surrey

\section{August 2012}

(C) Rowena Gee 2012 
ProQuest Number: 27558528

All rights reserved

INFORMATION TO ALL USERS

The quality of this reproduction is dependent upon the quality of the copy submitted.

In the unlikely event that the author did not send a complete manuscript and there are missing pages, these will be noted. Also, if material had to be removed, a note will indicate the deletion.

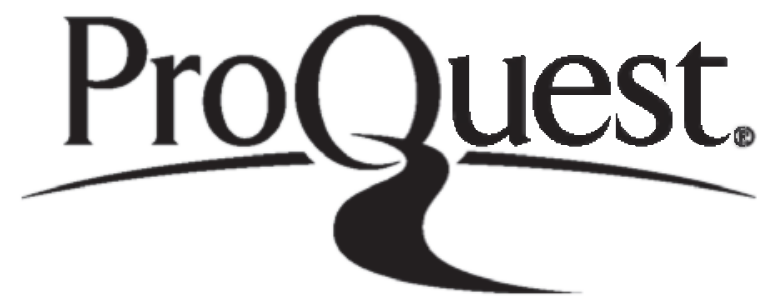

ProQuest 27558528

Published by ProQuest LLC (2019). Copyright of the Dissertation is held by the Author.

All rights reserved.

This work is protected against unauthorized copying under Title 17, United States Code Microform Edition (C) ProQuest LLC.

ProQuest LLC.

789 East Eisenhower Parkway

P.O. Box 1346

Ann Arbor, Ml $48106-1346$ 


\section{Statement of Originality}

"This thesis and the work to which it refers are the results of my own efforts. Any ideas, data, images or text resulting from the work of others (whether published or unpublished) are fully identified as such within the work and attributed to their originator in the text, bibliography or in footnotes. This thesis has not been submitted in whole or in part for any other academic degree or professional qualification. I agree that the University has the right to submit my work to the plagiarism detection service TurnitinUK for originality checks. Whether or not drafts have been so-assessed, the University reserves the right to require an electronic version of the final document (as submitted) for assessment as above."

- Rowena Gee (August 2012) 


\section{Abstract}

Statins inhibit 3-hydroxy-3-methylglutaryl coenzyme A reductase (HMGCR), the ratelimiting enzyme in cholesterol biosynthesis, and are widely used to treat hypercholesterolaemia, a risk factor for cardiovascular disease. Myopathy (muscle toxicity) is an adverse effect that is thought to occur due to the secondary effects of inhibiting HMGCR; however the mechanism of this myopathy is not fully understood. Therefore, the aim of this study was to use two human cell lines as an in vitro model for comparing the effects of a variety of statins on both cholesterol synthesis and protein prenylation between the liver, the intended target tissue for therapeutic benefit, and skeletal muscle, the major site of toxicity.

Initially, the differentiation of RD rhabdomyosarcoma muscle cells was characterised in terms of morphological and molecular changes that occur, using microscopy and TaqMan analysis respectively, and a 5-day cell differentiation period was chosen for use in later experiments. The initial hypothesis to then be tested was that the process of protein prenylation exhibits circadian variation, as has already been shown for cholesterol synthesis, and this might enable temporal separation between the cholesterol-lowering efficacy and the toxicity of statins. However, the ability of the RD cell line to establish and maintain circadian rhythmicity in vitro could not be verified and so the circadian contributions to cholesterol metabolism and protein prenylation in liver and muscle in this model could not be investigated.

Using the 3-(4,5-dimethylthiazol-2-yl)2,5-diphenyl tetrazolium bromide (MTT) assay, significant, dose-dependent reductions in cell viability were observed in both liver and muscle cells on treatment with each of the statins $(P=0.0041$, atorvastatin; $P<$ 0.0001 , all other statins, two-way analysis of variance (ANOVA)). However, greater cell toxicity was observed with the more lipophilic statins, simvastatin (acid), lovastatin (acid) and cerivastatin, and also in RD muscle cells compared to Huh7 liver cells. Taken together, these data represent the first comprehensive comparative analysis of statin toxicity in human liver and muscle cell lines. Cholesterol metabolism was then examined in these cells: Although cholesterol was present in all cell types, the levels in RD cells were somewhat lower than in Huh7 cells, probably due to much lower levels 
of HMGCR as measured at both the transcript and protein level; and 48 hour treatment with $10 \mu \mathrm{M}$ simvastatin and pravastatin, and $1 \mu \mathrm{M}$ cerivastatin, seemed to lead to a decrease in total cellular cholesterol.

Subsequently, protein was extracted from Huh7 and RD cells treated with simvastatin, cerivastatin or pravastatin, and then subjected to analysis by western blot to detect any changes in the prenylation status of selected small GTPases. Inhibition of prenylation was found by directly analysing changes in the unprenylated form of Rap1A, using a specific antibody, and by examining the reduction in membrane association of $\mathrm{H}$-Ras, M-Ras and Rab1A. Treatment with simvastatin and cerivastatin inhibited the prenylation of Rap1A, and seemed to also affect membrane association of the small GTPases H-Ras, M-Ras and Rab1A. In both muscle and liver cells the inhibition of prenylation of Rap1A, a Ras-related GTPase, was 10-fold more sensitive to treatment with cerivastatin than simvastatin. Inhibition of Rap1A prenylation in RD muscle cells was also 10 -fold more sensitive to simvastatin and 3 -fold more sensitive to cerivastatin treatment than in liver cells. Hydrophilic pravastatin, which showed the lowest toxicity in MTT assays, did not affect the prenylation of any of the small GTPases examined, as far as was detectable using western blot analysis.

In conclusion, this work has shown that RD muscle cells show both greater sensitivity to the toxic effects of statins and preferential disruption of protein prenylation in response to statin treatment when compared to Huh7 liver cells. This further supports a mechanism whereby disrupting production of intermediates in the cholesterol synthesis pathway, including the isoprenoids used in protein prenylation, is a significant cause of statin-induced muscle toxicity. This study also highlights the importance of examining the effects of drugs at both the target site of action and at the site of toxicity, rather than only studying one site in isolation, in order to gain insight into the mechanism of adverse drug effects. 


\section{Acknowledgements}

I have always loved science and studying the natural world, flitting between loves of biology, chemistry and maths. The path of a PhD has been in my mind for a long time, however it was only after having worked as a research assistant for a while that I felt ready (ha-ha) to take one on. I realised that I missed academia, and the challenge of wrestling with a problem to try and find the answer. Little did I realise how big a challenge it would turn out to be! There are so many people who have been such a huge support to me along this PhD path, who I would just like to take a moment to say thank you to.

Firstly I would like to say a huge thank you to Kate Plant, Jonathan Johnston and Nick Plant; I could not have asked for better supervisors. I really appreciate the way that you were all so patient with me, the way you encouraged me at those times that I did not think I could make it, yet how you also knew when I needed a push. I have become a better scientist and person because of you.

Thank you to The British Toxicology Society (BTS) and The University of Surrey's Centre for Toxicology for funding this research.

Thank you to all in the Molecular Toxicology group at Surrey. It has been great to spend the last few years working alongside you all. Thank you to Raj and Sam for helping me to settle in, teaching me all about cell culture and cell dosing, and for being so amazingly patient with me as I asked a million questions. Raj, I have particularly appreciated your handy equations! Thank you, Ciaran for your ability to keep us cheerful and make us all laugh in the office; and for your help, advice (and sympathy!) with western blotting and numerous other techniques. Thank you, Jenny for all of your technical help. Faizah, thank you for your help with using the statins, and thank you Mary and Karen for your help with learning how to do MTT assays. Thanks also go to the rest of the Mol Tox group, those still present and those who have since left to move on to other things: Jo, Heather, Izabel, Barbara, Mateus, Selma, Ellen, Noura, Shirri, Yib, Codou, Dile, Lisi, Alfred, Rita, Helen, Jason, Maryam, Lisa, Rugaia, Arnida, Fahad, Murat, and anyone else I may have unintentionally forgotten! Thank you also to all in the chronobiology group, particularly to Simone and Ana, to all those PhD students from other parts of the faculty (especially Etty) and the University who have helped me along the way, and to Julie Howarth for allowing me to use the digital camera from Histology for taking the cell photos. Thank you also to all who I had the opportunity to meet at conferences who gave me ideas to help direct this research, particularly Dr Daniel Antoine (MRC Centre for Drug Safety Science).

Margaret: where do I start thanking you?! I am so glad that we met in our induction week here at Surrey, and it has just been such a huge blessing to share the ups and downs of life with you over the last few years. Thank you.

Glyn: thank you for your friendship and for all the music. You know that I have always loved your songs and always considered it a huge privilege to be able to play music with you. We have had a lot of good times over the years! Special thanks for the times that you kept me company during parts of my overnight serum pulse experiments and for providing me with a place to write some of this thesis in June 2011.

Sarah $\mathrm{H}$ : thank you for all of those lunches at work; for keeping me sane and letting me rant at you, even though often you had no idea what I was talking about! 
There are so many other friends who I do not have space to name here, those in Guildford at Eternity Church, at the Surrey Christian Union and those who live further afield. You know who you are. I feel so blessed to know you all, and by your love, support and prayers.

I also do not know where to start thanking my Mum, Dad, brother and G'pa. Thank you so much for all of your love and support, and for putting up with me when I am sure that it can't be easy sometimes. I love you all so much, and am so glad to have you as my family.

And last, but most definitely not least, I could not write acknowledgements without acknowledging the most important person in my life - God: my father, my friend, my refuge, my saviour. You are the only one who sees the very deepest parts of me and yet still you keep loving me, being patient with me and encouraging me - that will never cease to amaze me. You have told me to be strong and courageous and not to be afraid or discouraged, for you are with me wherever I go (Joshua 1:9). At times that has been hard to hold onto but you have always brought me through to the other side, somehow. And I know that wherever I go from here, you will continue to go before me, with me and behind me. Thank you.

\section{Psalm 23 \\ (New Living Translation)}

The Lord is my shepherd; I have all that I need.

He lets me rest in green meadows;

He leads me beside peaceful streams.

He renews my strength.

He guides me along right paths,

bringing honour to his name.

Even when I walk through the darkest valley,

I will not be afraid,

for you are close beside me.

Your rod and your staff

protect and comfort me.

You prepare a feast for me

in the presence of my enemies.

You honour me by anointing my head with oil.

My cup overflows with blessings.

Surely your goodness and unfailing love will pursue me

all the days of my life,

and I will live in the house of the Lord

forever. 


\section{Contents}

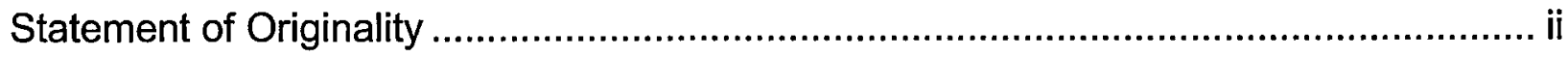

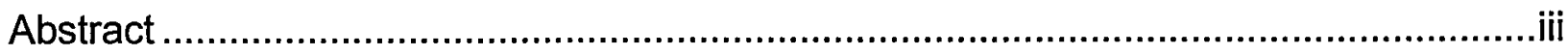

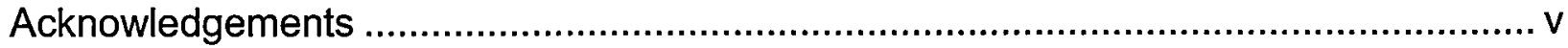

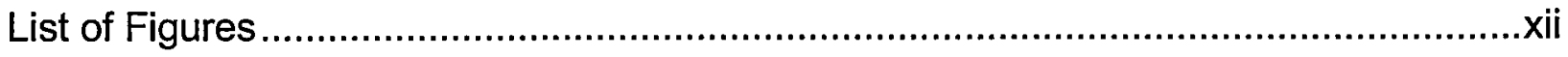

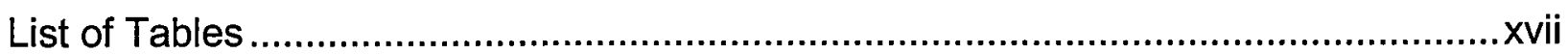

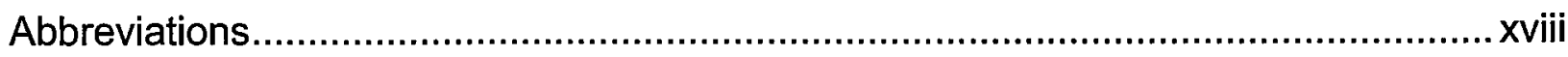

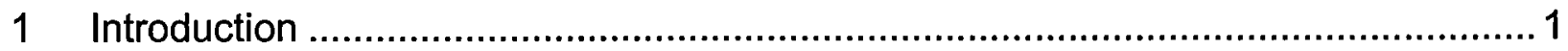

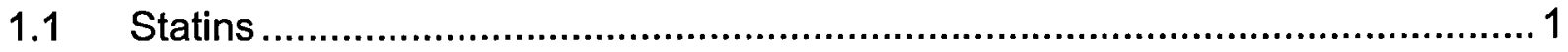

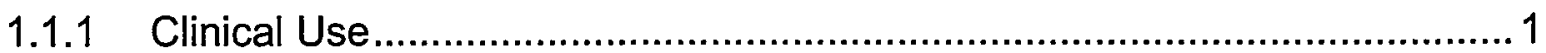

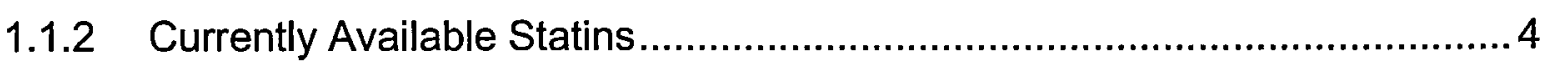

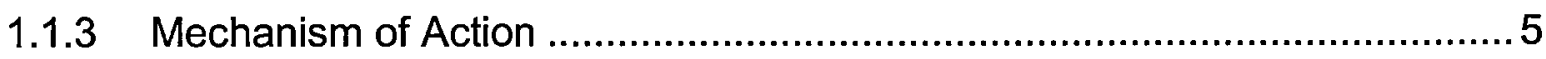

1.1.4 Similarities and Differences between the Statins .................................... 8

1.2 The Problem of Statin-Induced Myopathy ................................................... 10

1.2.1 Myopathy and its Incidence amongst Statin Users .................................. 10

1.2.2 Factors Affecting the Incidence of Statin-Induced Myopathy .................... 12

1.3 The Mechanism of Statin-Induced Myopathy …......................................... 14

1.3.1 Decreased Membrane Cholesterol ........................................................... 14

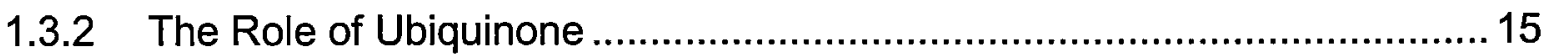

1.3.3 Dolichol and Glycosylation ............................................................... 16

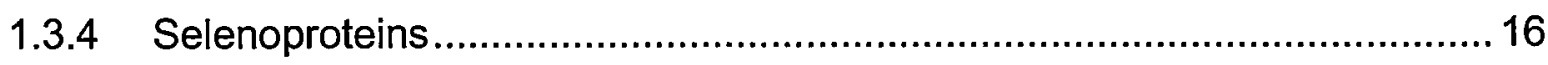

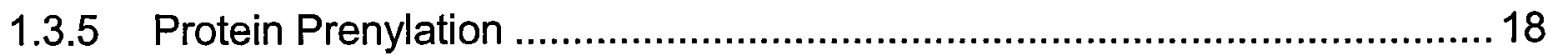

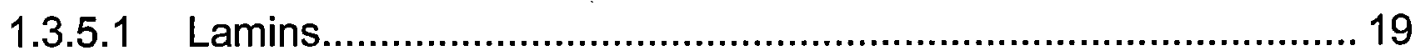

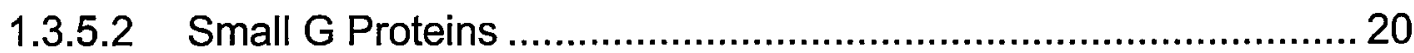

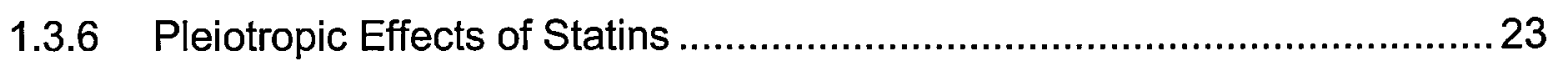

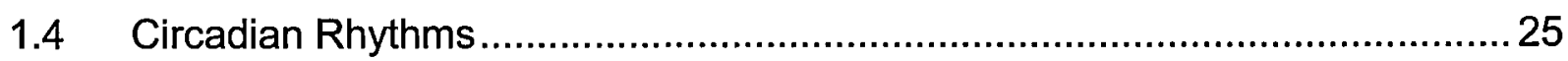

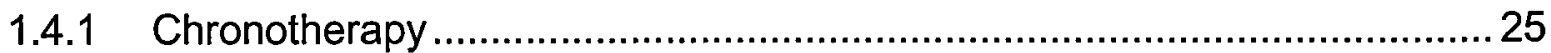

1.4.2 The Molecular Basis of Mammalian Circadian Rhythms ...........................27

1.4.3 Hierarchy of the Mammalian Circadian Timing System ............................2 29

1.4.4 Circadian Control of Cholesterol Metabolism...........................................31

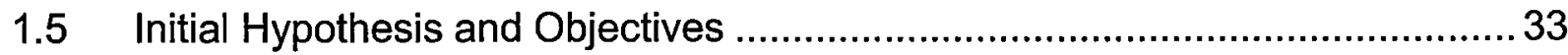




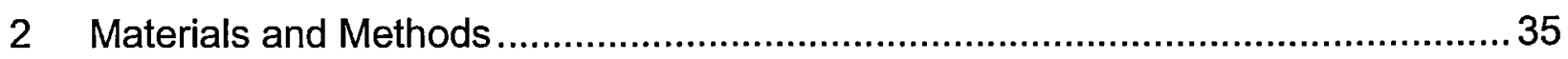

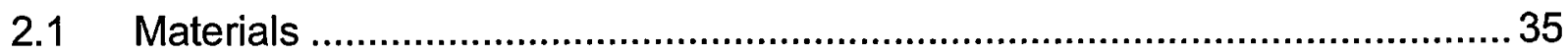

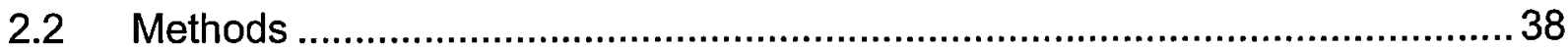

2.2.1 Cell Culture of Immortalised Cell Lines and Cell-Based Procedures .........38

2.2.1.1 RD Cell Culture ......................................................................... 38

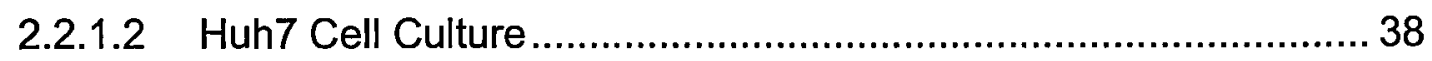

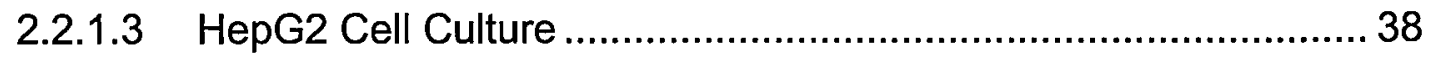

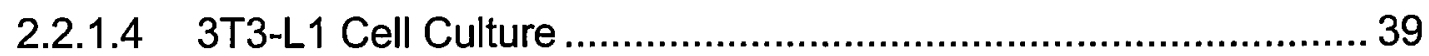

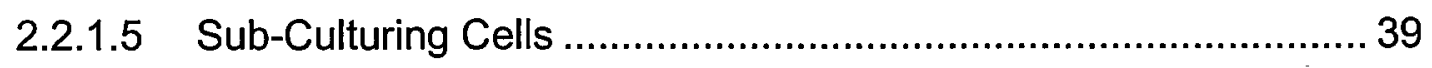

2.2.1.6 Counting Cells using a Haemocytometer ................................. 40

2.2.1.7 Storage of Cells in Liquid Nitrogen ............................................. 40

2.2.1.8 Recovery of Cells from Liquid Nitrogen .................................... 40

2.2.1.9 RD cell Differentiation ............................................................. 41

2.2.1.10 Synchronising Cells using a Serum Pulse ............................... 42

2.2.1.11 Treatment of Cells with Xenobiotics .......................................... 43

2.2.1.12 Assessing Cell Viability using the MTT Assay ........................... 44

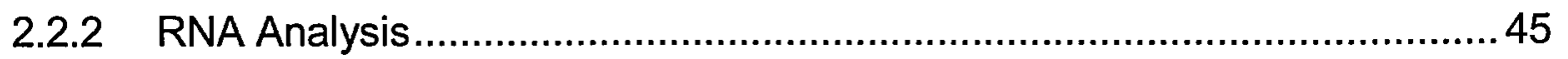

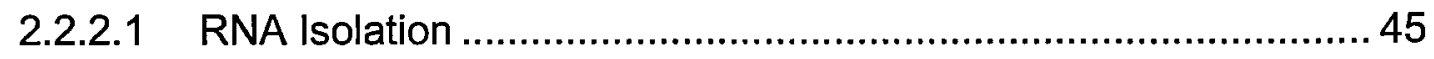

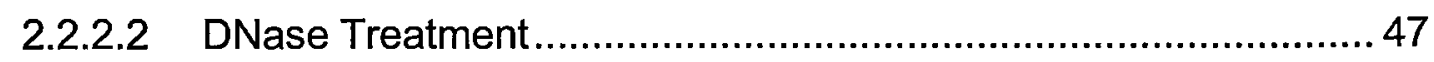

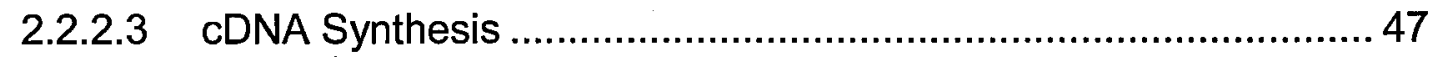

2.2.2.4 Quantitative Reverse Transcriptase Polymerase Chain Reaction47

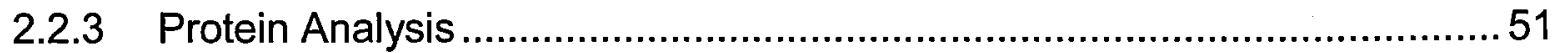

2.2.3.1 Total Protein Extraction using RIPA Buffer............................... 51

2.2.3.2 Membrane Protein Extraction ................................................. 51

2.2.3.3 Protein Quantification using the Lowry Method ......................... 52

2.2.3.4 Concentrating Protein Samples................................................. 53

2.2.3.5 Separation of Proteins by SDS-PAGE ................................... 53

2.2.3.6 Identification of Proteins by Western Blotting .............................. 54

2.2.3.7 Membrane Stripping and Reprobing .......................................56

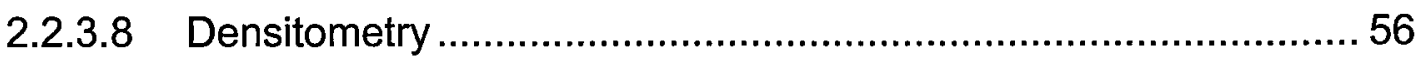

2.2.4 Measurement of Total Cellular Cholesterol Content ...............................56 
2.2.4.1 Extraction of Lipids from Cells ............................................... 57

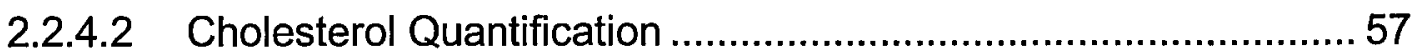

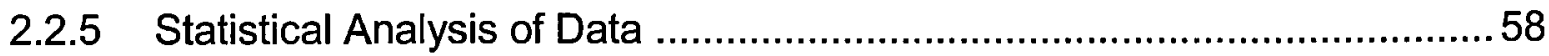

3 Characterisation of Human Liver and Muscle Cell Lines ....................................59

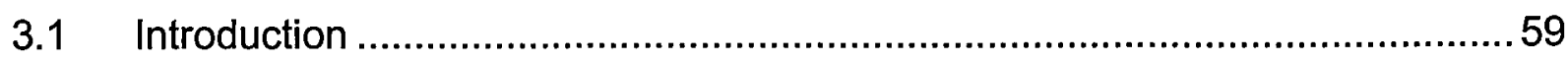

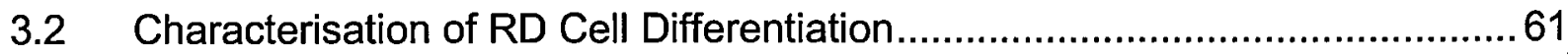

3.2.1 Expression of Muscle-Specific Markers ..................................................61

3.2.2 Expression of Cholesterol Metabolism-Related Genes..............................65

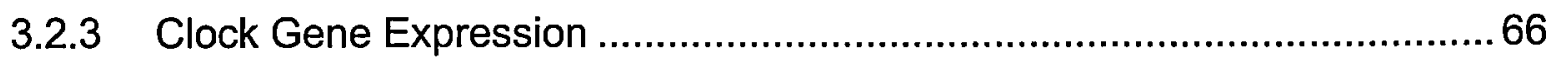

3.3 Characterisation of Circadian Rhythms .....................................................68

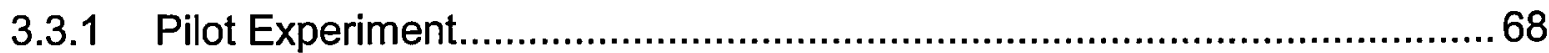

3.3.2 Circadian Analysis in Huh7 and RD Cells ............................................ 70

3.3.2.1 Analysis of Temporal Gene Expression in Huh7 Cells ................ 70

3.3.2.2 Analysis of Temporal Gene Expression in RD-D Cells................ 72

3.3.2.3 Normalisation of Gene Expression Data ................................... 74

3.3.2.4 Relative Abundance of Clock Genes......................................... 79

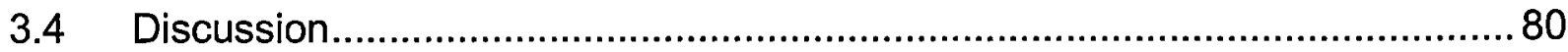

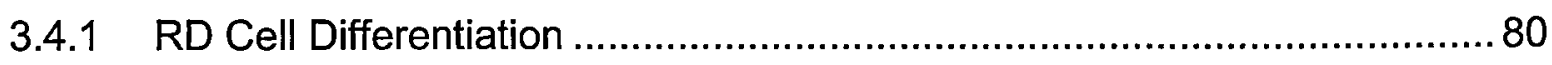

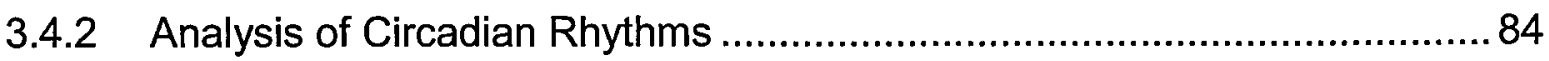

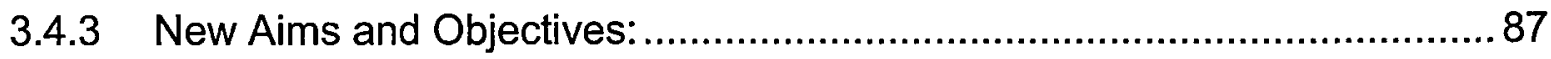

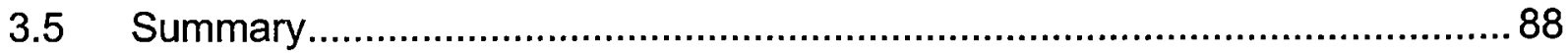

4 Differential Sensitivity of Liver and Muscle Cell Lines to Statin Treatment .............89 89

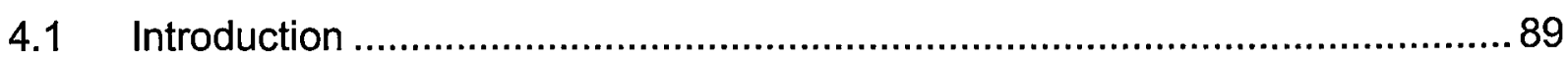

4.2 Characterisation of Statin-Mediated Cell Toxicity ........................................90

4.3 Analysis of Cholesterol Metabolism in Liver and Muscle Cells........................95

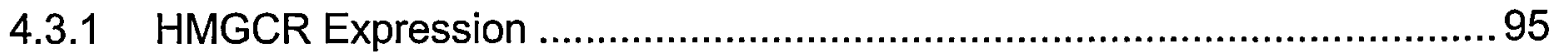

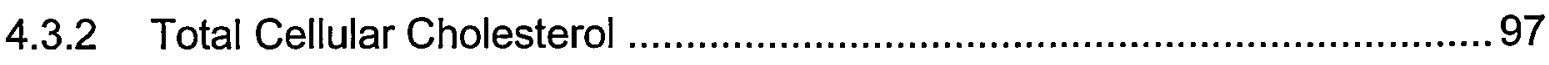

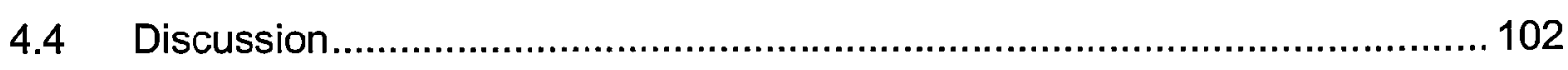

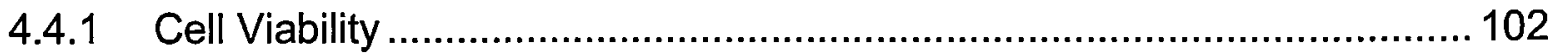

4.4.1.1 Differences between Liver and Muscle .................................... 102

4.4.1.2 The Effect of Different Statins and the Role of Statin Exposure 103 
4.4.2 Cholesterol Metabolism ................................................................... 105

4.4.2.1 Differences in HMGCR Expression ....................................... 105

4.4.2.2 The Effect of Statin Treatment on Total Cellular Cholesterol .... 106

4.4.3 Choice of Statin Concentration .............................................................. 109

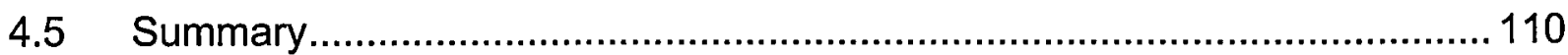

5 The Effects of Statins on Prenylation in Liver and Muscle Cells......................... 111

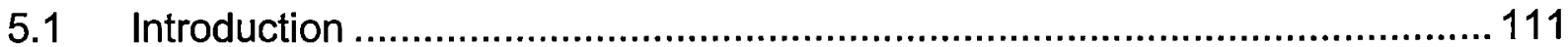

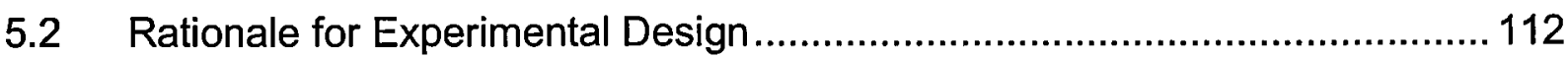

5.3 The Effects of Statins on Prenylation ..................................................... 114

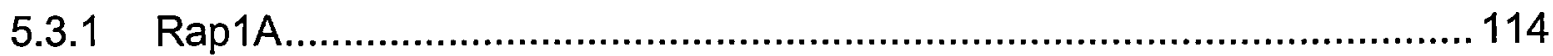

5.3.1.1 Dose-Dependent Effects of Statin Treatment on Rap1A Prenylation

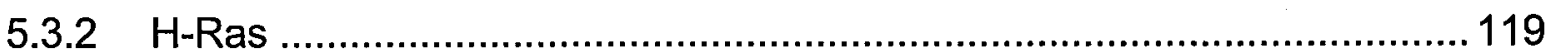

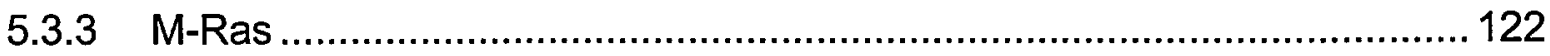

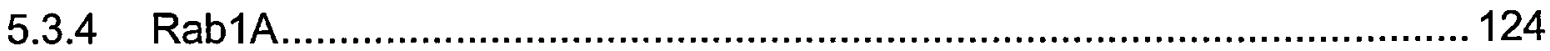

5.3.5 Lactate Dehydrogenase (LDH) .................................................... 128

5.4 The Effect of Statins on Prenyltransferase Expression .............................. 130

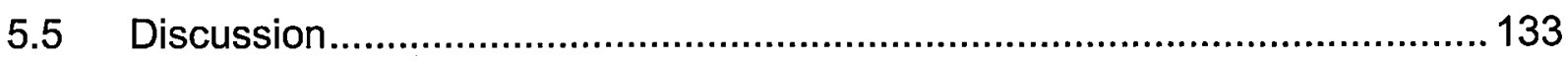

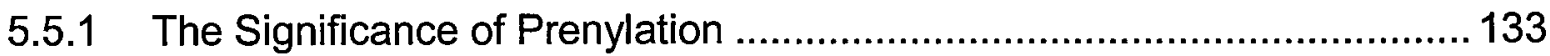

5.5.2 Prenylation and the Statins ............................................................ 133

5.5.2.1 Membrane Association of Small GTPases ............................... 134

5.5.2.2 Differential Disruption of Prenylation ..................................... 136

5.5.2.3 Prenyltransferase Inhibitors................................................ 137

5.5.3 Prenyltransferase Expression ......................................................... 138

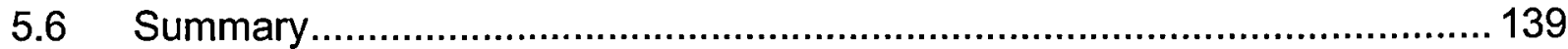

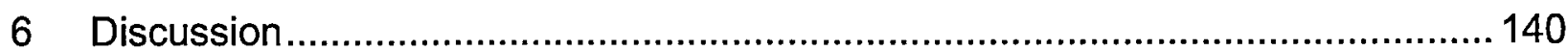

6.1 In Vitro Modelling of Statin-Induced Toxicity ............................................... 141

6.2 Prenylation and Muscle Physiology......................................................... 144

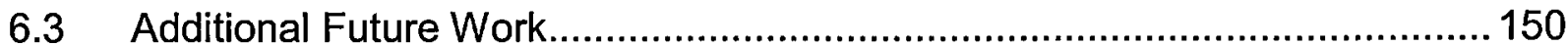

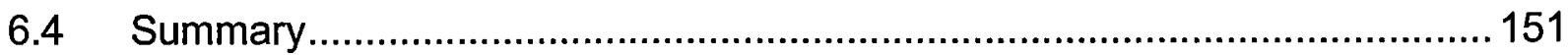

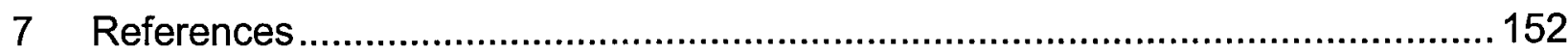


8 Appendices

8.1 Normalisation of Temporal Gene Expression Data from Pilot Serum Puise

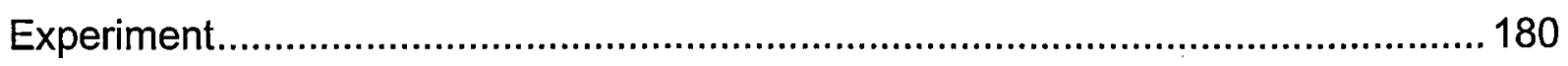

8.1.1 3T3-L1 Murine Preadipocyte Cell Line................................................. 180

8.1.2 HepG2 and Huh7 Human Liver Cell Lines............................................182

8.2 Additional Normalisations of Temporal Gene Expression Data from

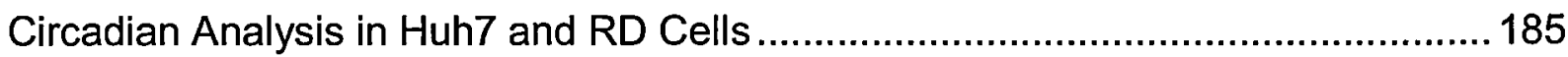

8.2.1 Normalisation of Temporal Gene Expression Data from Huh7 Cells ....... 185

8.2.2 Normalisation of Temporal Gene Expression Data from RD-D Cells ....... 187

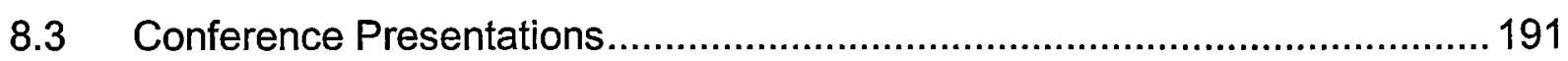




\section{List of Figures}

\section{Chapter 1}

Figure 1-1 The structure of cholesterol

5

Figure 1-2 The conversion of 3-hydroxy-3-methylglutaryl-CoA (HMG-CoA) to mevalonate is catalysed by the enzyme HMG-CoA reductase (HMGCR) ...................... 6

Figure 1-3 The mevalonate pathway: biosynthesis of cholesterol and other essential isoprenoids

Figure 1-4 Chemical structures of the different statins 8

Figure 1-5 The mammalian molecular oscillator.

Figure 1-6 Daily rhythmicity in key enzymes and receptors involved in the regulation of cholesterol levels or statin metabolism 31

\section{Chapter 2}

Figure 2-1 Protocol for differentiation of RD cells

Figure 2-2 Protocol for pilot serum pulse experiment

Figure 2-3 Protocol for serum pulse of Huh7 and RD cells

Figure 2-4 Example of how an agarose gel can be used to give an indication of RNA quality

\section{Chapter 3}

Figure 3-1 Morphological characterisation of RD muscle cell differentiation .62

Figure 3-2 Molecular characterisation of RD muscle cell differentiation 63

Figure 3-3 Protein expression of muscle-specific markers in Huh7 and RD cells. 64

Figure 3-4 Cholesterol metabolism during RD muscle cell differentiation 65 
Figure 3-5 Expression of clock genes during RD muscle cell differentiation 66

Figure 3-6 Temporal expression of PER2 and DBP in 3 different cell lines. 69

Figure 3-7 Temporal gene expression in Huh7 liver cells 71

Figure 3-8 Temporal gene expression in RD-D muscle cells. 73

Figure 3-9 Temporal gene expression of two different housekeeping genes in Huh7 and RD-D cells

Figure 3-10 Effect of normalisation of Huh7 TaqMan data using two different housekeeping genes

Figure 3-11 Effect of normalisation of RD-D TaqMan data using two different housekeeping genes .78

Figure 3-12 Comparison of gene expression in Huh7 liver and RD-D muscle cells 79

Figure 3-13 Regulation of skeletal myogenesis. 81

\section{Chapter 4}

Figure 4-1 Optimisation of time of incubation with MTT reagent .91

Figure 4-2 Cell viability in response to statin treatment .92

Figure 4-3 HMGCR gene expression in different cell types .95

Figure 4-4 HMGCR protein expression in different cell types. .96

Figure 4-5 Effect of $1 \mu \mathrm{M}$ statin treatment on total cellular cholesterol .98

Figure 4-6 Effect of $10 \mu \mathrm{M}$ statin treatment on total cellular cholesterol .99

Figure 4-7 Effect of control treatment on total cellular cholesterol in Huh7 cells 100

Figure 4-8 Effect of seeding density on total cellular cholesterol in Huh7 cells 101 


\section{Chapter 5}

Figure 5-1 Protein prenylation

Figure 5-2 Effect of statin treatment on Rap1A prenylation

Figure 5-3 Effect of prenylation inhibitors on Rap1A prenylation

Figure 5-4 Effect of simvastatin treatment on Rap1A prenylation

Figure 5-5: Effect of cerivastatin treatment on Rap1A prenylation

Figure 5-6 Effect of statin treatment on membrane association of H-Ras 120

Figure 5-7 Effect of prenylation inhibitors on membrane association of H-Ras

Figure 5-8 Effect of statin treatment on membrane association of M-Ras

Figure 5-9 Effect of prenylation inhibitors on membrane association of M-Ras.

Figure 5-10 Effect of statin treatment on membrane association of Rab1A 125

Figure 5-11 Effect of prenylation inhibitors on membrane association of Rab1A 126

Figure 5-12 Localisation of lactate dehydrogenase (LDH), in response to statin treatment

Figure 5-13 Localisation of $L D H$, in response to treatment with prenyltransferase inhibitors

Figure 5-14 Effect of statin treatment on expression of farnesyltransferase and geranylgeranyltransferase-I.

Figure 5-15 Effect of statin treatment on expression of geranylgeranyltransferase-II.. 132

Figure 5-16 Effect of prenylation inhibitors on expression of farnesyltransferase, geranylgeranyltransferase-I and geranylgeranyltransferase-II 


\section{Chapter 6}

Figure 6-1 Linking the molecular effects of the statins with muscle physiology and myopathy

\section{Appendices}

Figure 8-1 Temporal gene expression of two different housekeeping genes in 3T3-

L1 cells 180

Figure 8-2 Effect of normalisation of 3T3-L1 TaqMan data using two different housekeeping genes

Figure 8-3 Temporal gene expression of three different housekeeping genes in HepG2 and Huh7 cells

Figure 8-4 Effect of normalisation of HepG2 TaqMan data using three different housekeeping genes

Figure 8-5 Effect of normalisation of Huh7 TaqMan data using three different housekeeping genes

Figure 8-6 Effect of normalisation of Huh7 TaqMan data (PER1 and REV-ERBa) using two different housekeeping genes

Figure 8-7 Effect of normalisation of Huh7 TaqMan data (HMGCR) using two different housekeeping genes

Figure 8-8 Effect of normalisation of RD-D TaqMan data (BMAL1 and CRY1) using two different housekeeping genes

Figure 8-9 Effect of normalisation of RD-D TaqMan data (PER1 and REV-ERBa) using two different housekeeping genes

Figure 8-10 Effect of normalisation of RD-D TaqMan data (MYOD1 and MYOG) using two different housekeeping genes 
Figure 8-11 Effect of normalisation of RD-D TaqMan data (HMGCR) using two different housekeeping genes 


\section{List of Tables}

\section{Chapter 1}

Table 1-1 Percentage change in plasma concentration of low density lipoprotein (LDL) cholesterol with approved doses of eight statins in patients with hypercholesterolaemia

Table 1-2 Generic and most common brand names of statins in the United Kingdom

Table 1-3 Summary of the major differences between properties of the statins 9

Table 1-4 Reported cases of fatal rhabdomyolysis for all statins dispensed in the United States from when these products were launched until 2001

\section{Chapter 2}

Table 2-1 Suppliers of specialist materials used in this work .37

Table 2-2 Solvents used for xenobiotics. .44

Table 2-3 Human specific primer and probe sequences 49

Table 2-4 Mouse specific primer and probe sequences .50

Table 2-5 Antibody conditions for western blotting 55

\section{Chapter 4}

Table 4-1 IC50 values for the reduction in cellular viability on statin treatment..... .93

Table 4-2 Significance of cell type in determining response to statin treatment 94 


\section{Abbreviations}

3T3-L1 Mouse Preadipocyte Cell Line

$4 \mathrm{~S}$

ACTB

Scandinavian Simvastatin Survival Study

ADME

$\beta$-Actin

AMPK

Absorption, Distribution, Metabolism and Excretion

ANOVA

Adenosine Monophosphate-Activated Protein Kinase

APS

Analysis of Variance

ATCC

Ammonium Persulphate

$\mathrm{Bcl}-2$

American Type Culture Collection

BisTris

B-cell lymphoma 2

BMAL1

Bis-(2-hydroxyethyl)-imino-tris(hydroxymethyl)-methane

Bmal1

Brain and Muscle Arnt-Like Protein-1 (protein)

BMAL1 Reference specifically to the human gene

BME $\quad \beta$-Mercaptoethanol

bp

BSA

Base Pairs

$\beta-\operatorname{TrCP}$

Bovine Serum Albumin

BTS

Transducin Repeat Containing Protein

$\mathrm{C} 2 \mathrm{C} 12$

British Toxicology Society

$\mathrm{Ccg}$

Mouse Myoblast Cell Line

CDNA

Clock Controlled Gene

Ceriva

Copy DNA

CK1

Cerivastatin

CLOCK Circadian Locomotor Output Cycles Kaput

CoQ10 Coenzyme Q10 (also known as ubiquinone)

CRY Cryptochrome

CYP Cytochrome P450

CYP3A4 Cytochrome P450, Family 3, Subfamily A, Polypeptide 4

CYP7A1 Cholesterol-7 $\alpha$-Hydroxylase

CYP8B1 Sterol-12 $\alpha-$ Hydroxylase

DBP D site of Albumin Promoter (Albumin D-box) Binding Protein

DEC1 Deleted in Oesophageal Cancer 1 


$\begin{array}{ll}\text { DMEM } & \text { Dulbecco's Modified Eagle Medium } \\ \text { DMSO } & \text { Dimethyl Sulphoxide } \\ \text { DNA } & \text { Deoxyribonucleic Acid } \\ \text { dNTP } & \text { Deoxyribonucleotide Triphosphate }\end{array}$

ECACC European Collection of Cell Cultures

EDTA Ethylenediaminetetraacetic Acid

ER Endoplasmic Reticulum

FBS Foetal Bovine Serum

FBXL3 F-box and Leucine-Rich Repeat Protein 3

FDA US Food and Drug Administration

FOXO1 Forkhead Box 01

FPP Farnesylpyrophosphate

FT Farnesyltransferase

GAPDH Glyceraldehyde 3-Phosphate Dehydrogenase

GDP Guanosine Diphosphate

GGPP Geranylgeranylpyrophosphate

GGT-I Geranylgeranyltransferase type I

GGT-II Geranylgeranyltransferase type II

GSK GlaxoSmithKline

GTP Guanosine Triphosphate

GTPase Guanine Nucleotide-Binding Protein

HepG2 Human Hepatoblastoma Cell Line

HMG-CoA 3-hydroxy-3-methylglutaryl Coenzyme A

HMGCR 3-Hydroxy-3-Methylglutaryl Coenzyme A Reductase

HPLC High Performance Liquid Chromatography

H-RAS v-Ha-Ras Harvey Rat Sarcoma Viral Oncogene Homolog

Huh7 Human Hepatocellular Carcinoma 7 Cell Line

HRP Horse Radish Peroxidase

ID Inhibitor of Differentiation

IGF-1 Insulin-Like Growth Factor 1

JUPITER Justification for the Use of Statins in Primary Prevention: an Intervention Trial Evaluating Rosuvastatin

$\mathrm{kDa} \quad$ Kilodaltons

LC-MS Liquid Chromatography-Mass Spectrometry 


$\begin{array}{ll}\text { LDH } & \text { Lactate Dehydrogenase } \\ \text { LDL } & \text { Low-Density Lipoprotein } \\ \text { LDLR } & \text { Low-Density Lipoprotein Receptor } \\ \text { LXR } & \text { Liver-X-Receptor } \\ \text { MAPK } & \text { Mitogen-Activated Protein Kinase } \\ \text { MCK } & \text { Muscle Creatine Kinase } \\ \text { MEK } & \text { Mitogen-Activated Protein Kinase Kinase } \\ \text { MHC } & \text { Myosin Heavy Chain } \\ \text { M-RAS } & \text { Muscle RAS Oncogene Homolog } \\ \text { MRF } & \text { Myogenic Regulatory Factor } \\ \text { mRNA } & \text { Messenger RNA } \\ \text { MTT } & \text { 3-(4,5-Dimethylthiazol-2-yl)2,5-Diphenyl Tetrazolium Bromide } \\ \text { MYH3 } & \text { Myosin Heavy Chain 3 } \\ \text { MYOD1 } & \text { Myogenic Differentiation 1 } \\ \text { MYOG } & \text { Myogenin } \\ \text { NADPH } & \text { Nicotinamide Adenine Dinucleotide Phosphate } \\ \text { NEAA } & \text { Non-Essential Amino Acids } \\ \text { NPAS2 } & \text { Neuronal PAS Domain Protein 2 } \\ \text { NSAIDS } & \text { Non-Steroidal Anti-Inflammatory Drugs } \\ \text { OAT } & \text { Organic Anion Transporter } \\ \text { OATP } & \text { Organic Anion Transporting Polypeptide } \\ \text { PAR } & \text { Proline and Acidic Amino Acid-Rich } \\ \text { PBS } & \text { Phosphate Buffered Saline } \\ \text { PER } & \text { Period } \\ \text { PI3K } & \text { Phosphatidylinositol 3-Kinase } \\ \text { PP } & \text { Pyrophosphate } \\ \text { Prava } & \text { Pravastatin } \\ \text { PVDF } & \text { Polyvinylidene Difluoride } \\ \text { qPCR } & \text { Quantitative Polymerase Chain Reaction } \\ \text { RD } & \text { Human Embryonic Rhabdomyosarcoma Cell Line } \\ \text { RD-U } & \text { Undifferentiated RD Cells } \\ \text { RD-D } & \text { Differentiated RD Cells } \\ \text { REP } & \text { Rab Escort Protein } \\ \text { REV-ERBa } & \text { Reverse Erythroblastosis Virus } \alpha \\ & \\ \text { MY }\end{array}$




\begin{tabular}{|c|c|}
\hline RIPA & RadiolmmunoPrecipitation Assay \\
\hline RNA & Ribonucleic Acid \\
\hline ROR & Retinoic Acid-Related Orphan Nuclear Receptor \\
\hline RRE & Retinoic Acid-Related Orphan Receptor Response Element \\
\hline rRNA & Ribosomal RNA \\
\hline RT-PCR & Reverse Transcriptase Polymerase Chain Reaction \\
\hline SCF & Skp1-Cul1-F-Box-Protein \\
\hline SCN & Suprachiasmatic Nucleus \\
\hline SD & Standard Deviation \\
\hline SDS-PAGE & Sodium Dodecyl Sulphate Polyacrylamide Gel Electrophoresis \\
\hline Sec-tRNA & Selenocysteine tRNA \\
\hline SEM & Standard Error of the Mean \\
\hline SEPN1 & Selenoprotein $\mathrm{N}$ \\
\hline Simva & Simvastatin (acid) \\
\hline SLC01B1 & Solute Carrier Organic Anion Transporter Family, Member 1B1 \\
\hline SNP & Single Nucleotide Polymorphism \\
\hline SREBP & Sterol Regulatory Element Binding Protein \\
\hline T25 & $25 \mathrm{~cm}^{2}$ Vented Cell Culture Flask \\
\hline T75 & $75 \mathrm{~cm}^{2}$ Vented Cell Culture Flask \\
\hline TAE & Tris Acetate EDTA \\
\hline TBP & TATA-Binding Protein \\
\hline TBST & Tris-Buffered Saline-Tween 20 \\
\hline TCA & Trichloroacetic Acid \\
\hline TCEP & Tris(2-carboxyethyl)phosphine $\mathrm{HCl}$ \\
\hline TEMED & Tetramethylethylenediamine \\
\hline TPA & 12-O-Tetradecanoyl Phorbol-13-Acetate \\
\hline tRNA & Transfer Ribonucleic Acid \\
\hline UK & United Kingdom \\
\hline US & United States \\
\hline VN & Volume per volume \\
\hline WN & Weight per Volume \\
\hline WOSCOPS & West of Scotland Coronary Prevention Study \\
\hline
\end{tabular}




\section{Introduction}

\subsection{Statins}

\subsubsection{Clinical Use}

Cardiovascular diseases are the leading cause of death worldwide, causing $27 \%$ and $32 \%$ of all deaths of men and women respectively (World Health Organisation 2008). Lowering cholesterol in the form of low-density lipoprotein (LDL) is a common strategy in the fight against cardiovascular disease since an elevated concentration of cholesterol in this form is known to be one of the most important risk factors (Brown and Goldstein 2004).

The statin class of drugs is currently the first line of treatment for hypercholesterolaemia, after numerous large clinical trials have demonstrated the benefits of their use (Liao 2002; Ong 2005). In particular, the $4 S$ trial (Scandinavian Simvastatin Survival Study 1994) and the West of Scotland Coronary Prevention Study (WOSCOPS) (Shepherd et al. 1995) were two trials in the 1990s that were very significant in first demonstrating the advantages of statin treatment (Ong 2005). It has been shown that statins are very effective at lowering blood LDL levels (Weng et al. 2010); in short-term studies LDL cholesterol reductions of $17-55 \%$ have been measured in patients, depending on the dose and the particular statin used (Table 1-1). Widespread use of statins has also led to a decrease in the rates of morbidity and mortality due to atherosclerosis (Stossel 2008). 


\begin{tabular}{|c|c|c|}
\hline Statin & $\begin{array}{c}\text { Dose } \\
\text { (mg/day) }\end{array}$ & $\begin{array}{c}\text { LDL } \\
\text { Cholesterol (\%) }\end{array}$ \\
\hline Lovastatin & $\begin{array}{l}20 \\
40 \\
80\end{array}$ & $\begin{array}{l}-29 \\
-32 \\
-48\end{array}$ \\
\hline Simvastatin & $\begin{array}{l}10 \\
20 \\
40 \\
80\end{array}$ & $\begin{array}{l}-28 \\
-35 \\
-41 \\
-46\end{array}$ \\
\hline Pravastatin & $\begin{array}{l}10 \\
20 \\
40\end{array}$ & $\begin{array}{l}-19 \\
-24 \\
-34\end{array}$ \\
\hline Fluvastatin & $\begin{array}{c}20 \\
40 \\
80^{*}\end{array}$ & $\begin{array}{l}-17 \\
-23 \\
-36\end{array}$ \\
\hline Atorvastatin & $\begin{array}{l}10 \\
20 \\
40 \\
80\end{array}$ & $\begin{array}{l}-38 \\
-46 \\
-51 \\
-54 \\
\end{array}$ \\
\hline Rosuvastatin $^{1,2}$ & $\begin{array}{c}5 \\
10 \\
20 \\
40 \\
\end{array}$ & $\begin{array}{l}-42 \\
-47 \\
-53 \\
-54 \\
\end{array}$ \\
\hline Pitavastatin $^{1}$ & $\begin{array}{l}1 \\
2 \\
4 \\
8 \\
\end{array}$ & $\begin{array}{l}-34 \\
-42 \\
-47 \\
-55 \\
\end{array}$ \\
\hline Cerivastatin & $\begin{array}{l}0.3 \\
0.4 \\
0.8\end{array}$ & $\begin{array}{l}-31 \\
-36 \\
-45\end{array}$ \\
\hline
\end{tabular}

${ }^{*}$ Extended-release formulation (-36\%); $40 \mathrm{mg}$ twice daily $(-32 \%)$

Table 1-1 Percentage change in plasma concentration of low density lipoprotein (LDL) cholesterol with approved doses of eight statins in patients with hypercholesterolaemia

Data from (Chong et al. 2001), other than where indicated, obtained from short-term studies ranging from 4-10 weeks in duration. ${ }^{1}$ Data from (Mukhtar et al. 2005), obtained from studies of 6 weeks (rosuvastatin, $20 \mathrm{mg}$ and $40 \mathrm{mg}$ ) or 12 weeks (pitavastatin) in duration. ${ }^{2}$ Data from (Blasetto et al. 2003) obtained from a study of 12 weeks in duration (rosuvastatin, $5 \mathrm{mg}$ and 10mg). 
Statins are used to treat both those with established cardiovascular disease (secondary prevention) and those who are healthy but designated 'high risk' of a cardiovascular event (primary prevention). However, treatment is continually expanding to include those at lower risk (Hlatky 2008). This is partially due to the results of several recent clinical trials, which have demonstrated the benefits of the use of statins in primary prevention (Brugts et al. 2009). One such example is the Justification for the use of Statins in Primary Prevention: an Intervention Trial Evaluating Rosuvastatin (JUPITER); in which comparison with a placebo group showed a reduction in the number of major cardiovascular events, in healthy men and women with low LDL levels but elevated levels of the inflammatory biomarker high-sensitivity C-reactive protein who were treated with rosuvastatin (Hlatky 2008; Ridker et al. 2008). There is currently debate over the potential benefits of statin treatment even for those who are considered at low risk and would not be considered for such intervention under current guidelines (Mihaylova et al. 2012; Taylor et al. 2011).

For this reason, as well as from looking at recent trends (Stagnitti 2008), the use of statins seems set to only continue to increase in the coming years. Therefore, it is all the more important to have an in-depth understanding of the mechanisms by which these drugs exert their cholesterol-lowering and other non-cholesterol related effects on the body, and to understand the factors regulating these effects. 


\subsubsection{Currently Available Statins}

Since the first inhibitor of cholesterol synthesis was discovered by Akira Endo in 1977 (Steinberg 2006), a range of related compounds have been isolated and synthesized, and collectively these cholesterol lowering drugs are known as the statins. The currently available statins can be divided into two classes - those isolated from fungi or derived from fungal products (type 1 statins) and those which are completely synthetic (type 2). Those in the former category include lovastatin, simvastatin and pravastatin. Those in the latter category include fluvastatin, atorvastatin, rosuvastatin, pitavastatin and cerivastatin (Shitara and Sugiyama 2006). Generic names and the most common UK brand names of statins are listed in Table 1-2.

\begin{tabular}{|ll|}
\hline Generic Name & Brand Name (Manufacturer) \\
\hline Atorvastatin & Lipitor (Pfizer) \\
Cerivastatin & Lipobay (Bayer) \\
Fluvastatin & Lescol (Novartis) \\
Lovastatin & Mevacor (Merck), Altocor, Altoprev \\
Pitavastatin & \\
Pravastatin & Livazo, Alipza (Kowa Pharmaceutical) \\
Rosuvastatin & Lipostat (Bristol-Myers Squibb) \\
Simvastatin & Crestor (AstraZeneca) \\
\hline
\end{tabular}

Table 1-2 Generic and most common brand names of statins in the United Kingdom Information taken from the British National Formulary (Joint Formulary Committee 2008). ${ }^{1}$ Cerivastatin was withdrawn from the worldwide market in 2001 (Bellingham 2001); ${ }^{2}$ Pitavastatin is not yet commercially available in the UK (Kowa Pharmaceutical Europe 2011).

Pitavastatin is the newest statin; it has been available in Japan since 2003 (Chapman 2010) and is now being marketed as Livazo in an increasing number of countries around the world, with the first European launch having taken place in Spain in 2011 (Kowa Pharmaceutical Europe 2011). In July 2004 simvastatin became available over-the-counter in the United Kingdom (UK), as a $10 \mathrm{mg} / \mathrm{day}$ dose, with a licence for sale to those at moderate (10-15\%) ten-year risk of a first coronary event (National Institute for Health and Clinical Excellence 2006). This controversial decision made the UK the first country to have a non-prescription 
statin (Abrams 2005). Cerivastatin was withdrawn from the worldwide market in 2001 and is therefore no longer commercially available (Bellingham 2001).

\subsubsection{Mechanism of Action}

Cholesterol plays a vital role in the body and is essential for normal cell function: It is a major constituent of eukaryotic cell membranes, where its sterol ring structure provides rigidity (Figure 1-1). This means that the amount of cholesterol present in a membrane is important in determining the properties of the membrane. Cholesterol is also the precursor to steroid hormones (such as oestrogen, progesterone, testosterone and glucocorticoids), vitamin $\mathrm{D}$ and bile salts. However, in excess it can also contribute to the build up of lipid deposits and hardening of the arteries in atherosclerosis, which is thought to often lead to cardiovascular disease (Shitara and Sugiyama 2006; Voet and Voet 2004).

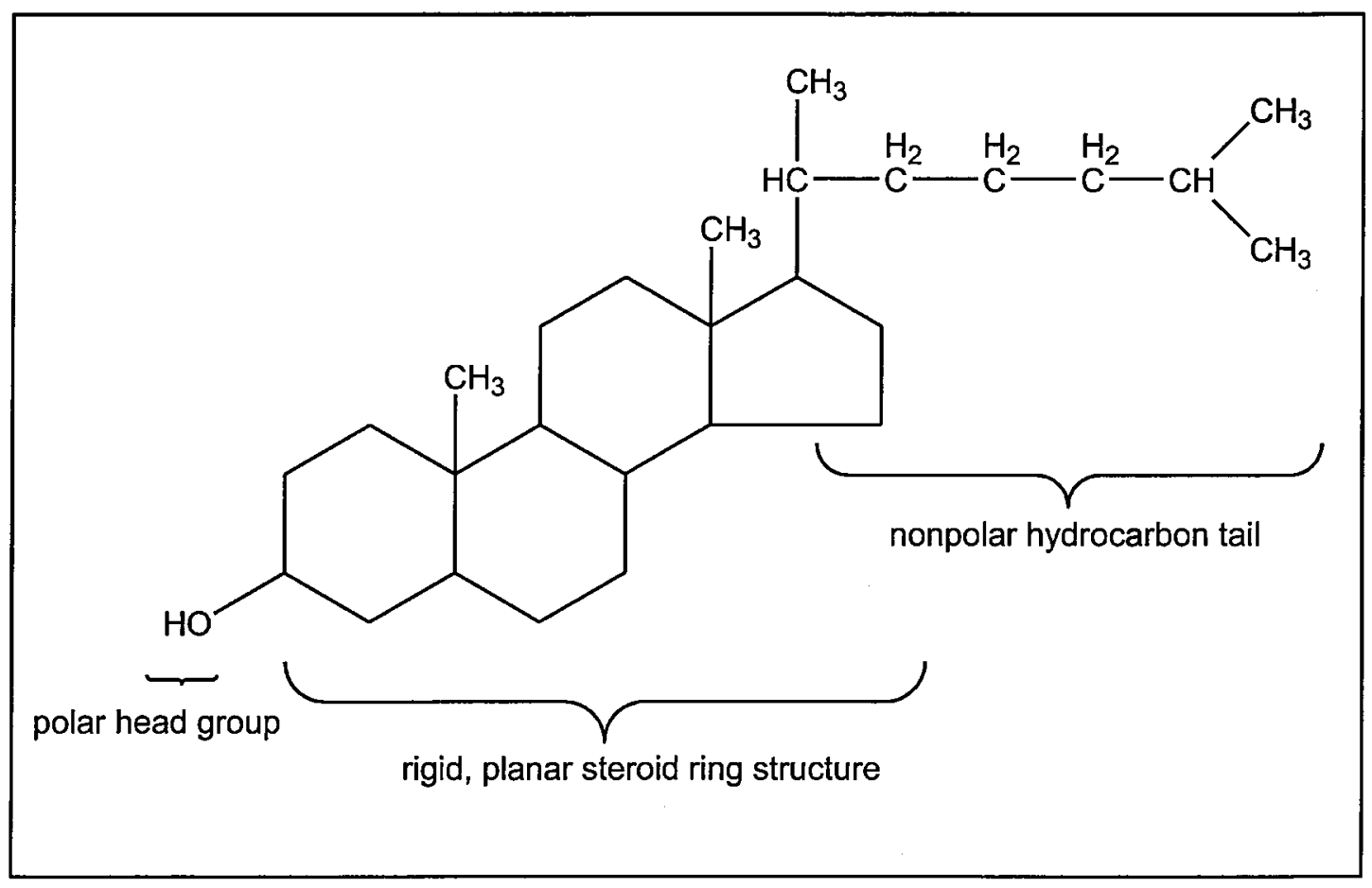

Figure 1-1 The structure of cholesterol

Cholesterol has a polar head group, a sterol ring structure that provides rigidity to cell membranes, and a nonpolar hydrocarbon tail. (Voet and Voet 2004).

There are two main sources of cholesterol in the body: cholesterol from the diet and cholesterol that has been synthesized internally. Although almost all cells are 
able to synthesize cholesterol, the liver is the main site of cholesterol biosynthesis in the body and is therefore the main target organ of all of the statins (Catapano 2007).

Cholesterol biosynthesis is a complex pathway involving many enzymes and intermediates. All statins act by competitively inhibiting the enzyme 3-hydroxy-3methylglutaryl coenzyme A (HMG-CoA) reductase (HMGCR) (Figure 1-2), which catalyses the first committed, and rate-limiting, step of cholesterol biosynthesis (Shitara and Sugiyama 2006). The resulting decrease in de novo cholesterol synthesis leads to upregulation of the LDL receptor (LDLR), through the activation of cholesterol-sensing transcription factor sterol regulatory element binding protein (SREBP) (Brown and Goldstein 1997). The increase in LDLR then leads to increased uptake of cholesterol, in the form of LDL, from the bloodstream into cells, resulting in an overall reduction in plasma LDL levels (Brown and Goldstein 2004).

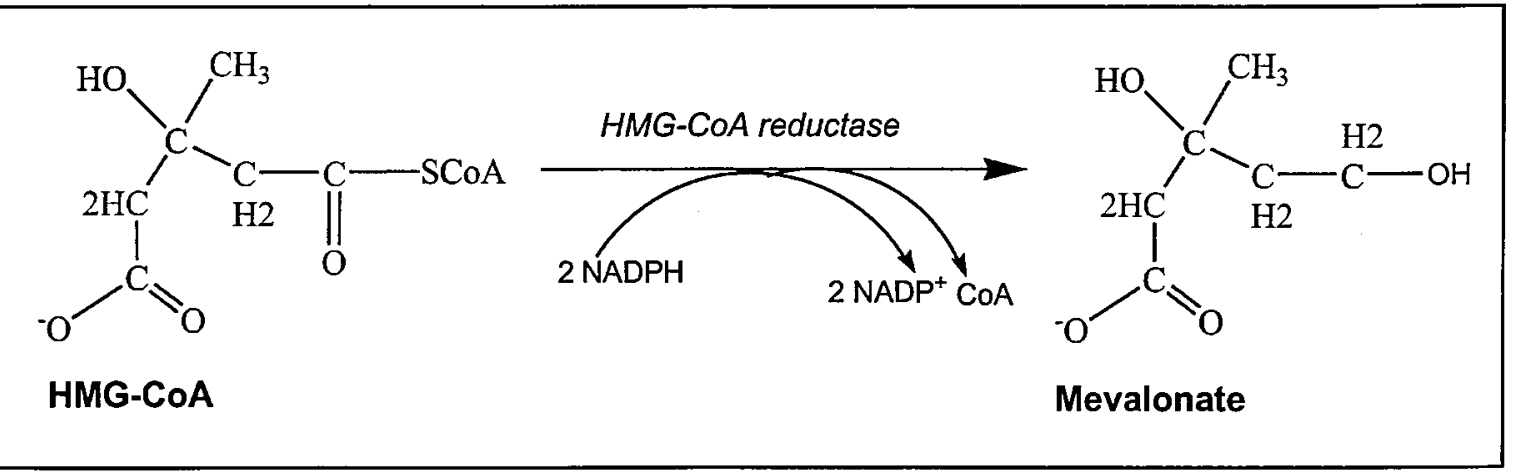

Figure 1-2 The conversion of 3-hydroxy-3-methylglutaryl-CoA (HMG-CoA) to mevalonate is catalysed by the enzyme HMG-CoA reductase (HMGCR)

This reaction is a reduction, requiring NADPH (nicotinamide adenine dinucleotide phosphate), and is the rate-limiting step in the biosynthesis of cholesterol. It is also the step where statins exert their effect by acting as competitive inhibitors of HMGCR. (Voet and Voet 2004).

The mevalonate pathway (Figure 1-3), the branched pathway that cholesterol biosynthesis is a part of, uses common precursors to also synthesize a number of essential isoprenoids other than cholesterol. These include dolichol, isopentenylpyrophosphate, farnesylpyrophosphate (FPP) and geranylgeranylpyrophosphate (GGPP). Statin inhibition of HMGCR affects the 
level of all common precursors and hence levels of these other isoprenoids. It is this which is thought to underlie the major adverse effects of statins (Baker 2005).

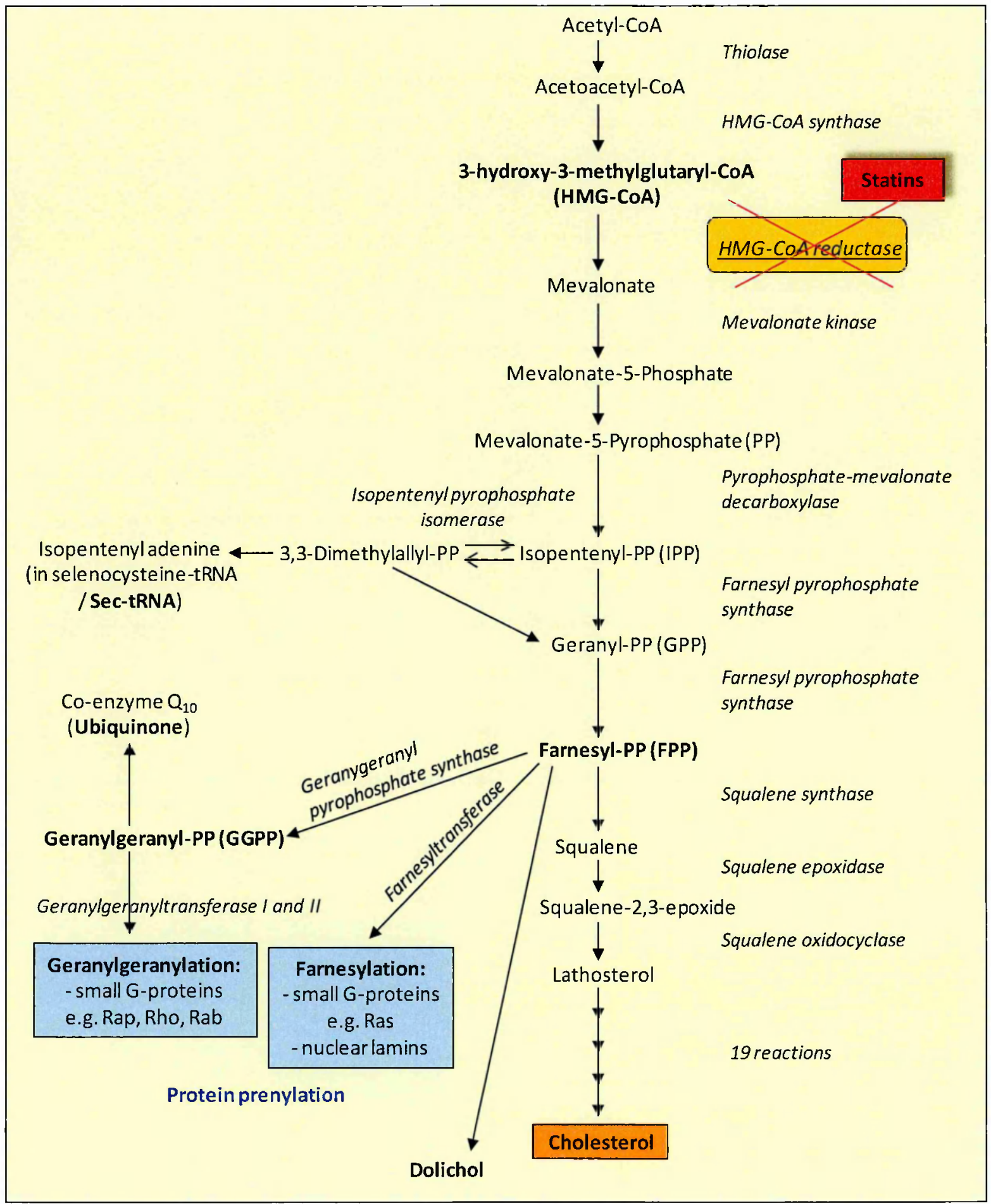

Figure 1-3 The mevalonate pathway: biosynthesis of cholesterol and other essential

\section{isoprenoids}

Statin inhibition of the rate-limiting enzyme HMGCR affects levels of not only cholesterol but also of sec-tRNA, ubiquinone, dolichol and prenylated proteins. Dolichol synthesis also uses isopentenylPP, but arrow not shown for diagram simplicity. Figure produced using the following references: (Baker 2005; Goldstein and Brown 1990; Lane and Beese 2006; McTaggart 2006; Shitara and Sugiyama 2006; Vaklavas et al. 2009; Voet and Voet 2004). 


\subsubsection{Similarities and Differences between the Statins}

All of the statins have an HMG-like moiety, enabling them to tightly bind to the catalytic site of HMGCR and block access for the substrate HMG-CoA, resulting in potent, competitive inhibition of HMGCR (Figure 1-4). However, there are structural differences between statins that result in differences in their physiological properties (Table 1-3). This includes differences in their modes of binding, which can be seen from the crystal structures that have been solved for a portion of the enzyme bound to each of six different statins (Istvan and Deisenhofer 2001). In general the newer statins exhibit a higher affinity for HMGCR and have more potent inhibitor effects (Shitara and Sugiyama 2006).

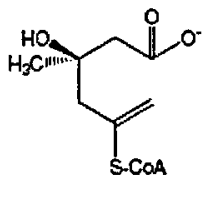

HMG-COA

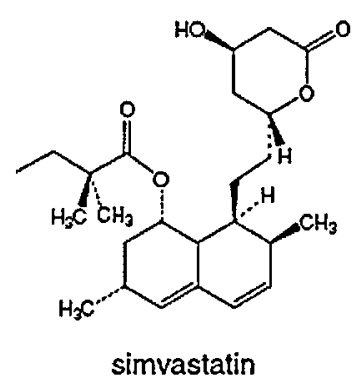

simvastatin

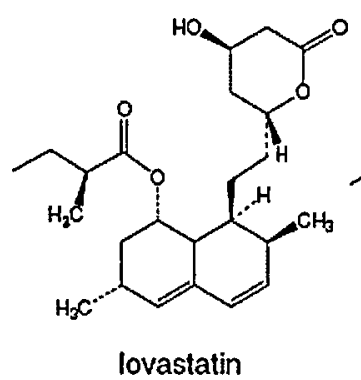

lovastatin<smiles>CC[C@H](C)C(=O)O[C@H]1C[C@H](O)C=C2C=C[C@H](C)[C@H](CCC(O)CC(O)CC(=O)O)[C@H]2[C@H]1C</smiles>

pravastatin

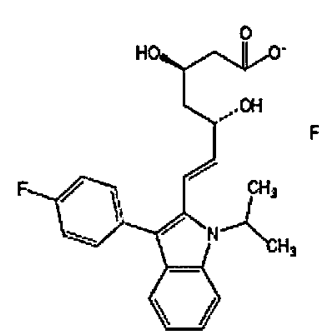

fluvastatin

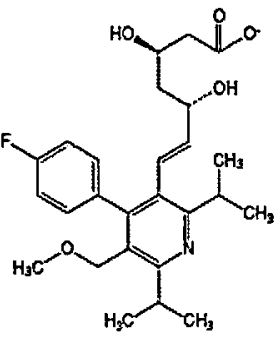

cerivastatin

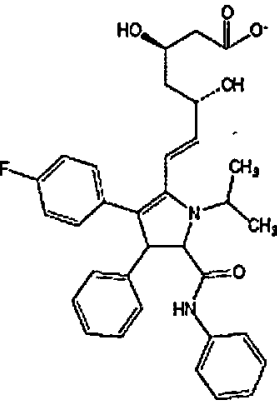

atorvastatin

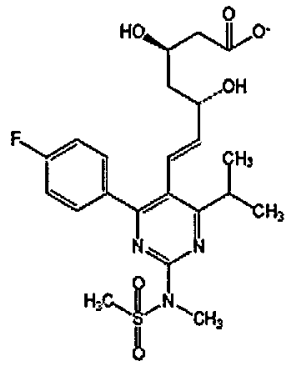

rosuvastatin

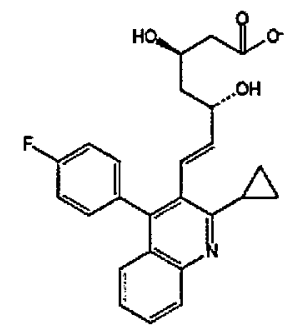

pitavastatin

Figure 1-4 Chemical structures of the different statins

All of the statins have an HMG-like moiety, which can either be in the lactone form (simvastatin and lovastatin) or in the open acid form (all other statins). However, there are also many differences in structure which account for the different physiological properties between statins. Figure reproduced from (Shitara and Sugiyama 2006). 


\begin{tabular}{|c|c|c|c|c|c|c|c|c|}
\hline Statin & $\begin{array}{c}\text { Simvastatin } \\
\text { (Simvastatin acid) }\end{array}$ & $\begin{array}{l}\text { Lovastatin } \\
\text { (Lovastatin acid) }\end{array}$ & Cerivastatin & Fluvastatin & Atorvastatin & Pitavastatin & Rosuvastatin & Pravastatin \\
\hline $\log \mathrm{D}(\mathrm{pH} 7.0)^{1}$ & \multirow[t]{2}{*}{$4.4(1.88)$} & \multirow[t]{2}{*}{$3.91(1.51)$} & 2.32 & 1.75 & 1.53 & 1.5 & \multirow{3}{*}{$\begin{array}{l}-0.25--0.50 \\
\text { Hydrophilic }\end{array}$} & \multirow{3}{*}{$\begin{array}{c}-0.47 \\
-0.75--1.00 \\
\text { Hydrophilic }\end{array}$} \\
\hline $\log \mathrm{D}(\mathrm{pH} 7.4)^{1}$ & & & $1.50-1.75$ & $1.00-1.25$ & $1.00-1.25$ & & & \\
\hline Lipophilicity & Lipophilic & Lipophilic & Lipophilic & Lipophilic & Lipophilic & Lipophilic & & \\
\hline $\begin{array}{c}\text { Uptake }^{2} \\
\text { Passive diffusion? } \\
\text { Active transport? }\end{array}$ & $\begin{array}{c}\text { Yes } \\
\text { (OATP1B1) }\end{array}$ & $\begin{array}{c}\text { Yes } \\
\text { (OATP1B1) }\end{array}$ & $\begin{array}{c}\text { Yes } \\
\text { OATP1B1 }\end{array}$ & $\begin{array}{c}\text { Yes } \\
\text { Unknown }\end{array}$ & $\begin{array}{c}\stackrel{?}{\text { OATP1B1 }} \\
\text { OATP2B1 (LM) }\end{array}$ & $\begin{array}{c}? \\
\text { OATP1B1 } \\
\text { (OATP1B3) }\end{array}$ & $\begin{array}{c}\text { No } \\
\text { OATP1B1 } \\
\text { OATP1B3 } \\
\text { OATP2B1 (L/M) }\end{array}$ & $\begin{array}{c}\text { No } \\
\text { OATP1B1 } \\
\text { OATP2B1 (LM) } \\
\text { OATP1A4 (M) } \\
\text { OAT3(M) }\end{array}$ \\
\hline \multirow[t]{4}{*}{ Metabolism $^{3}$} & \multirow{4}{*}{$\begin{array}{l}\text { CYP3A4 } \\
\text { CYP3A5 }\end{array}$} & \multirow[t]{4}{*}{ CYP3A4 } & \multirow{4}{*}{$\begin{array}{l}\text { (CYP3A4) } \\
\text { CYP2C8 }\end{array}$} & \multirow[t]{4}{*}{ CYP2C9 } & \multirow[t]{4}{*}{ CYP3A4 } & (CYP3A4) & CYP2C9 & CYP3A4 \\
\hline & & & & & & CYP2A9 & & Sulphation \\
\hline & & & & & & CYP2C9 & & \\
\hline & & & & & & Minimally; slow & Minimaily & Minimally \\
\hline Half-life (hours) $^{4}$ & 3 & $3-4$ & $2-3$ & $<1$ & 14 & 11 & 19 & 1.8 \\
\hline Bioavaliability $(\%)^{4}$ & $<5$ & $<5$ & 60 & 24 & 12 & $>60$ & 20 & 17 \\
\hline Year introduced $^{5}$ & 1991 & 1987 & $\begin{array}{c}1997 \\
\text { (withdrawn 2001) }\end{array}$ & 1993 & 1996 & $\begin{array}{c}2003 \text { (Japan) } \\
2011 \text { (EU) }\end{array}$ & 2003 & 1991 \\
\hline
\end{tabular}

Table 1-3 Summary of the major differences between properties of the statins

${ }_{1}^{1}$ (Shitara and Sugiyama 2006)

2 (Kitamura et al. 2008; Knauer et al. 2010; Sakamoto et al. 2008; Shitara and Sugiyama 2006;

Takeda et al. 2004)

${ }^{3}$ (Chong et al. 2001; Mukhtar et al. 2005; Vaklavas et al. 2009)

${ }^{4}$ (AstraZeneca 2007; Chong et al. 2001; Mukhtar et al. 2005)

${ }^{5}$ (AstraZeneca 2007; Recordati 24 October 2008; Staffa et al. 2002)

LogD values reflect the lipophilicity of a statin, with simvastatin and lovastatin being the most lipophilic (yellow), and rosuvastatin and pravastatin the least lipophilic (orange). The newest statins, atorvastatin, pitavastatin and rosuvastatin, appear to have the longest half-lives in the body. Bioavailability refers to the percentage of the drug that becomes available at the target site in the body, for example after absorption, and uptake refers to hepatic transport other than where noted: $\mathrm{L} / \mathrm{M}=$ liver and muscle, $\mathrm{M}=$ exclusively muscle transport. OATP = organic anion transporting polypeptides; OAT = organic anion transporter; CYP = Cytochrome P450.

The statins exhibit varying degrees of lipophilicity and therefore have different uptake mechanisms (Table 1-3). Simvastatin and lovastatin are the only statins that are administered as prodrugs in an inactive form, containing a lactone ring that is converted into the open acid form in the body. This means that simvastatin and lovastatin are the most lipophilic; they can cross lipid bilayers by passive diffusion and can therefore become widely distributed to a variety of tissues. In contrast, pravastatin and rosuvastatin are hydrophilic and rely on transporters for uptake into hepatocytes. The other statins have intermediate physicochemical properties (Hamelin and Turgeon 1998). Studies have demonstrated that transport of statins is mediated by various members of the organic anion transporting polypeptide 
(OATP) and organic anion transporter (OAT) families (Hsiang et al. 1999; Sakamoto et al. 2008; Takeda et al. 2004; Tokui et al. 1999). In particular OATP1B1, a transporter exclusive to liver, contributes to varying degrees to the hepatic uptake of many of the statins (Kitamura et al. 2008).

The statins are also metabolised differently by the liver (Table 1-3). The cytochrome P450 (CYP) family is a large family of haem-containing monooxygenase enzymes that catalyse many reactions involved in drug metabolism. CYP3A4 (cytochrome P450, family 3, subfamily A, polypeptide 4) is thought to be involved in the metabolism of approximately half of all drugs in current use (Takiguchi et al. 2007), and this includes simvastatin, lovastatin and atorvastatin (Bolego et al. 2002). Cerivastatin is metabolised by CYP2C8 as well as CYP3A4 and fluvastatin, alone amongst the statins, is metabolised by CYP2C9. Pitavastatin, rosuvastatin and pravastatin undergo minimal metabolism by CYP enzymes and are therefore not susceptible to many of the metabolism drug-drug interactions in the same way as other statins (Shitara and Sugiyama 2006). Both cerivastatin and atorvastatin have metabolites that contribute towards their lipidlowering effects (Chong et al. 2001).

All of these differences impact on statin prescribing choices, depending on the needs of each individual patient based on their risk profile (Chong et al. 2001). They potentially also impact upon the incidence of adverse effects (Bolego et al. 2002).

\subsection{The Problem of Statin-Induced Myopathy}

\subsubsection{Myopathy and its Incidence amongst Statin Users}

Statins are generally considered to be effective and relatively safe drugs, and are well tolerated by most (Law and Rudnicka 2006). However some patients do experience side effects, the most significant of which is myopathy. Myopathy is a general term used to refer to any disease of the muscles, which encompasses everything ranging from mild fatigue and minor muscle aches, pains, cramps and 
weakness to complete collapse of the muscle structure in rhabdomyolysis, which requires hospitalisation and in rare cases can prove fatal (Harper and Jacobson 2007).

Muscle complaints, including rhabdomyolysis, have been reported with all statins (Bolego et al. 2002) and rhabdomyolysis-related death has been reported with all statins other than fluvastatin (Table 1-4). It was the high number of deaths caused by rhabdomyolysis following treatment with cerivastatin in the US that prompted its withdrawal from the market in 2001 (Bellingham 2001). After cerivastatin, the next highest number of fatal cases has been reported in patients taking lipophilic drugs simvastatin and lovastatin; and in fact, due to increased risk of myopathy in general the highest dose of $80 \mathrm{mg}$ simvastatin is now no longer recommended, other than in patients who have already had this treatment for more than 12 months with no adverse muscle effects (Gotto and Moon 2012). Fluvastatin and pravastatin seem to produce the fewest cases of rhabdomyolysis; however these statins are also less effective at lowering blood cholesterol than some of the other statins (Table 1-1).

\begin{tabular}{|c|c|c|c|c|c|c|c|}
\hline & Lovastatin & Pravastatir & Simvastatin & Fluvastatin & Atorvastati & Cerivastatin & Total \\
\hline Date Approved & $08 / 1987$ & 10/1991 & $12 / 1991$ & $12 / 1993$ & $12 / 1996$ & $06 / 1997$ & - \\
\hline Fatal Cases of Rhabdomyolysis & 19 & 3 & 14 & 0 & 6 & 31 & 73 \\
\hline $\begin{array}{l}\text { No. prescriptions dispensed } \\
\text { since marketing began (million) }\end{array}$ & 99.197 & 81.364 & 116.145 & 37.392 & 140.360 & 9.815 & 484.273 \\
\hline $\begin{array}{l}\text { Reporting Rate (per } 1 \text { million } \\
\text { prescriptions) }\end{array}$ & 0.19 & 0.04 & 0.12 & 0 & 0.04 & 3.16 & 0.15 \\
\hline
\end{tabular}

Table 1-4 Reported cases of fatal rhabdomyolysis for all statins dispensed in the United States from when these products were launched until 2001

Fatal rhabdomyolysis has been reported with all statins, other than fluvastatin, however different statins have different reporting rates. The highest reporting rate of rhabdomyolysis was with cerivastatin, prompting its withdrawal from the market in 2001, with the next highest number of cases seen with simvastatin and lovastatin. Table reproduced from: (Staffa et al. 2002). Reported fatal cases of rhabdomyolysis are those reported to the US Food and Drug Administration (FDA) before 2001 and where death resulted either directly or indirectly from rhabdomyolysis. Reporting rate is the number of fatal cases divided by the number of prescriptions. 
Myalgia, muscle weakness and aches without elevation of creatine kinase, has been estimated to occur in 10-15\% of all statin patients (Mohaupt et al. 2009) and rhabdomyolysis incidence has been estimated at 3.4 per 100,000 person-years (with statins other than cerivastatin) (Law and Rudnicka 2006). However, it is difficult to accurately estimate the incidence of all statin-induced myopathy as it is well recognised that the less severe muscle effects are under-reported, and that incidence of myopathy in controlled clinical trials is artificially depressed, partly because many of those who would be more prone to statin-induced myopathy are not included in these trials (Vaklavas et al. 2009). It has also been suggested that cases of rhabdomyolysis are similarly under-reported (McAdams et al. 2008).

\subsubsection{Factors Affecting the Incidence of Statin-Induced Myopathy}

Statin-induced myopathy is known to be dose-dependent (Law and Rudnicka 2006) and any interaction which leads to an increase in statin exposure is a risk factor (Kitamura et al. 2008; Knauer et al. 2010; Sidaway et al. 2009).

The lipophilicity of statins such as simvastatin and lovastatin has been suggested to account for their higher incidence of adverse muscular side effects, due to their high rates of passive diffusion into all tissue, including muscle (Vaklavas et al. 2009). However, transporters such as OAT1, OATP1A4 and OATP2B1 have been reported to be expressed in muscle tissue and recognise statins as substrates, and could therefore play a role in determining muscle statin exposure (Table 1-3). The importance of these transporters in the development of muscle toxicity is not yet entirely clear (Knauer et al. 2010; Shitara and Sugiyama 2006; Sidaway et al. 2009), although it has been shown that some genetic polymorphisms affecting transporters, and therefore drug disposition, can affect patient risk of developing myopathy. One such example is a single nucleotide polymorphism (SNP) in the solute carrier organic anion transporter family, member 1B1 (SLCO1B1) gene, encoding the transporter OATP1B1, that has been associated with a higher risk of myopathy in response to simvastatin treatment (Link et al. 2008; Voora et al. 2009). Due to these findings a diagnostic test has now been launched, enabling 
the use of SLCO1B1 genotyping to identify those at high risk (Isis Innovation 2012).

Myopathy risk is also increased by drug interactions that can occur when statins are administered as part of combination therapy, particularly with drugs that can affect statin metabolism and therefore statin exposure (Knauer et al. 2010). Many drugs, including cyclosporine, azole antifungals, macrolides and calcium channel blockers, can inhibit the activity of CYP3A4, an enzyme important for the metabolism of many of the statins (Table 1-3). In fact, it has been estimated that interactions with drugs known to inhibit CYP3A4 activity underlie $60 \%$ of rhabdomyolysis cases associated with simvastatin, lovastatin or atorvastatin (Vaklavas et al. 2009). Similarly, fluvastatin may interact with inhibitors of CYP2C9, such as omeprazole and ritonavir (Chong et al. 2001), and with other substrates of CYP2C9, such as warfarin, fluconazole and diclofenac (Bolego et al. 2002).

The risk of rhabdomyolysis is substantially higher on co-treatment with a statin and a fibrate, particularly gemfibrozil (Harper and Jacobson 2010; Law and Rudnicka 2006; Mastaglia and Needham 2012). Gemfibrozil has been shown to inhibit CYP2C8, one of the enzymes involved in the metabolism of cerivastatin, which could account for the higher incidence of rhabdomyolysis in patients treated with both cerivastatin and gemfibrozil. Gemfibrozil is also a substrate of CYP3A4 and can inhibit the glucuronidation of statins (Prueksaritanont et al. 2002).

There are many patient characteristics which are thought to affect myopathy risk. These include several underlying medical conditions that can predispose a patient towards developing statin-induced myopathy, such as hepatic dysfunction, hypothyroidism and metabolic muscle diseases. Women and those of more advanced age are thought to be at higher risk of experiencing statin-induced myopathy (Harper and Jacobson 2010). In addition lifestyle choices, such as 
performing strenuous exercise and the consumption of alcohol, can also increase risk.

Time of dosing may impact upon tolerability of statins, as has already been demonstrated with a number of other drugs such as non-steroidal antiinflammatory drugs (NSAIDS) and cancer therapeutics, due to the impact of circadian rhythms on wide-ranging aspects of physiology (Levi and Schibler 2007). This will be discussed further in section 1.4 .

\subsection{The Mechanism of Statin-Induced Myopathy}

There continues to be much debate over the precise mechanism of statin-induced myopathy. Co-treatment with statin and mevalonate, the product of HMGCR, has been shown to prevent the development of myopathy in vivo (Westwood et al. 2008) and so the current consensus seems to be that the decreased production of essential isoprenoids in the mevalonate pathway is the most likely underlying cause of myopathy (Baker 2005; Mastaglia and Needham 2012; Sathasivam 2012; Vaklavas et al. 2009). There is varying evidence for the involvement of each of the products and intermediates of this pathway (Vaklavas et al. 2009).

\subsubsection{Decreased Membrane Cholesterol}

There is evidence to suggest that a decreased level of cholesterol is not in itself the cause of myopathy, although this was initially thought to be the case (Baker 2005; Nishimoto et al. 2003). Skeletal myopathy is associated with mevalonic aciduria, a disorder caused by deficiency of mevalonate kinase which is an enzyme important at an early stage of the cholesterol biosynthesis pathway (Figure 1-3). In contrast, syndromes caused by deficiencies in terminal enzymes (distal enzymopathies) in cholesterol biosynthesis do not show myopathy among their clinical manifestations (Baker 2005).

In addition, inhibitors of squalene synthase do not cause muscle toxicity in vitro (Flint et al. 1997) and they have in fact shown some protective effect on statininduced myopathy (Nishimoto et al. 2007). Unlike statins, they do not lead to a 
decrease in mevalonate (Elsayed and Evans 2008) since squalene synthase is the first enzyme downstream of the branch point in the mevalonate pathway and hence is the first enzyme solely committed to cholesterol synthesis (Charlton-Menys and Durrington 2008). There has therefore been considerable interest in squalene synthase inhibitors (Nishimoto et al. 2007; Nishimoto et al. 2003; Tavridou et al. 2006) however TAK-475 (lapaquistat), the only squalene synthase inhibitor to have progressed as far as phase III clinical trials in Europe and the United States, was recently discontinued from clinical development (Takeda 28 March 2008).

Consequently, it seems more likely that myopathy results from reductions in products and intermediates of the mevalonate pathway other than cholesterol. This means that potential candidates could include ubiquinone, dolichol, selenocysteine tRNA and the isoprenoids farnesylpyrophosphate (FPP) and geranylgeranylpyrophosphate (GGPP).

\subsubsection{The Role of Ubiquinone}

Ubiquinone (also known as coenzyme Q10, coQ10) is synthesized from GGPP, in a multistep side-branch of the mevalonate pathway (Figure 1-3), and its role in myopathy is unclear. CoQ10 acts as an electron carrier during oxidative phosphorylation and so is essential for mitochondrial energy production (Traustadottir et al. 2008). It has therefore been proposed that statin inhibition of coQ10 production could lead to myopathy due to muscle mitochondrial dysfunction (Marcoff and Thompson 2007; Sathasivam 2012).

It is well known that statins decrease plasma levels of coQ10, however it has yet to be shown conclusively that levels are also lowered in muscle and that lower muscle mitochondrial coQ10 leads to myopathy (Marcoff and Thompson 2007). Several clinical trials investigating the effects of coQ10 supplementation alongside statin therapy have proved inconclusive (Schaars and Stalenhoef 2008) and evidence thus far seems to suggest that coQ10 depletion is only likely to play a 
major role in myopathy in patients who already have depleted levels of coQ10 due to pre-existing conditions (Oh et al. 2007; Schaars and Stalenhoef 2008).

\subsubsection{Dolichol and Glycosylation}

Statins have been shown in vitro to affect $\mathrm{N}$-linked glycosylation (Mullen et al. 2010; Siddals et al. 2004), the most common form of glycosylation in mammals (Martin-Rendon and Blake 2003). Glycosylation, the addition of oligosaccharides to polypeptides to form glycoproteins, is an important co-translational covalent modification for the correct biological functioning of many proteins. Dolichol, a product of the mevalonate pathway, is vital in this process as it acts as a carrier for the 14 sugar oligosaccharide that is the precursor of all $\mathrm{N}$-linked oligosaccharide chains (Baker 2005; Vaklavas et al. 2009).

It is thought that a reduction in dolichol synthesis could result in myopathy due to an impaired response to growth factors such as insulin-like growth factor-1 (IGF-1). IGF-1 is well known to have proliferative effects in skeletal muscle, as well as a role in muscle fibre regeneration (myoregeneration) after injury (Barton et al. 2012), and statins have been shown to affect the $\mathrm{N}$-linked glycosylation and processing of IGF receptors in adipocytes (Siddals et al. 2004).

Many other cellular proteins could additionally be affected, for example the glycoprotein dystroglycan which mediates communication between the extracellular matrix and the cytoskeleton. This process is thought to also be important in myoregeneration (Cohn et al. 2002) and defects in posttranslational processing of dystroglycan have been linked to a variety of muscle disorders (Martin-Rendon and Blake 2003; Muntoni et al. 2004).

\subsubsection{Selenoproteins}

There are estimated to be approximately 25 human selenoproteins, which are proteins containing selenium usually in the form of selenocysteine (Muttenthaler and Alewood 2008). Selenocysteine is considered to be the $21^{\text {st }}$ amino acid in ribosome-mediated protein synthesis (Stadtman 1996) and its incorporation into 
proteins in selenoprotein synthesis is mediated by selenocysteine tRNA (Sec tRNA). Sec tRNA inserts a selenocysteine where there is a UGA codon in the messenger RNA. UGA is usually a stop codon; therefore incorrect decoding by sec tRNA can lead to premature termination of translation and production of truncated proteins.

It is known that isopentylation of adenosine 37 in sec tRNA is important for efficient decoding. This modification is catalysed by the enzyme isopentenyl pyrophosphate: tRNA isopentenyltransferase and uses isopentenyl-PP, an intermediate of the mevalonate pathway, as a substrate. Lovastatin treatment has been shown to lead to a reduction of sec tRNA with this modification and to reduce the ability of cells to synthesize selenoproteins (Warner et al. 2000).

Evidence for the potential importance of this selenoprotein deficiency for statin myopathy is that mutations in the gene for selenoprotein N (SEPN1) have been linked to a range of congenital muscular disorders. Selenoprotein $N$ is an endoplasmic reticulum glycoprotein postulated to be important for regulating intracellular calcium release via the ryanodine receptor intracellular calcium release channel; its correct function is therefore thought to be important for muscle fibre formation (myogenesis) and for myoregeneration (Jurynec et al. 2008; Petit et al. 2003).

Further evidence is provided by reports that statin-induced myopathy shares many similarities with myopathy resulting from selenium deficiency (Moosmann and Behl 2004). In addition, statin treatment has been shown to lead to a reduction in the activity of the selenoprotein glutathione peroxidase, leading to increased oxidative stress in human HepG2 liver cells (Kromer and Moosmann 2009). It is therefore possible that deficiency in selenoprotein synthesis could likewise lead to an increased vulnerability to oxidative stress in muscle. 


\subsubsection{Protein Prenylation}

Protein prenylation is known to be a very important process physiologically and it is thought that prenylated proteins account for up to $2 \%$ of total cellular protein. Prenylation involves the post-translational covalent addition of the prenyl groups farnesyl (15-carbon) or geranylgeranyl (20-carbon) to cysteine residues at or near the C-terminus of proteins via thioether linkages. The addition of these lipophilic groups is thought to be essential for the biological function of many proteins, by enabling them to anchor to cell membranes and hence specifying their cellular localisation. This is important for cellular protein-protein interactions and membrane-associated protein trafficking (McTaggart 2006). These prenyl groups are donated by the isoprenoids farnesylpyrophosphate (FPP) or geranylgeranylpyrophosphate (GGPP) respectively and, since both are intermediates in the mevalonate pathway, statin use is thought to lead to a decrease in protein prenylation.

There are three distinct protein prenyltransferase enzymes that catalyse the addition of prenyl groups to proteins: farnesyltransferase (FT), geranylgeranyltransferase-I (GGT-I) and geranylgeranyltransferase-II (GGT-II). FT and GGT-I both catalyse the addition of a prenyl group to the cysteine of a Cterminal CAAX protein motif, (where $C$ is cysteine, $A$ is an aliphatic residue and $X$ can be a wide variety of different amino acids (McTaggart 2006)). They are therefore known as the CAAX prenyltransferases. They are zinc metalloenzymes and heterodimers that share an $\alpha$ subunit but have different $\beta$ subunits. It is thought that the $\beta$ subunits of these enzymes contain the substrate binding sites (Casey and Seabra 1996) and that it is these different $\beta$ subunits which provide the different substrate specificities (Terry et al. 2006). GGT-II (also known as Rab GGT) consists of distinct $\alpha$ and $\beta$ subunits. In a complex with Rab escort protein (REP) it catalyses the addition of geranylgeranyl groups onto two cysteine residues in the $\mathrm{C}$-terminal recognition sequences $\mathrm{CC}$ or $\mathrm{CXC}$, or a range of other similar $\mathrm{C}$ terminal motifs (Maurer-Stroh et al. 2003). 
Substrates of FT include small $G$ protein Ras and the nuclear lamins, and substrates of GGT-I include Ras-related GTPases, such as those of the Ras, Rap, and Rho subfamilies, and the $y$ subunits of heterotrimeric G-proteins (Casey and Seabra 1996; Lane and Beese 2006). GGT-II catalyses the addition of prenyl groups onto small G proteins of the Rab subfamily (Casey and Seabra 1996; Leung et al. 2006). There is increasing evidence to suggest that disruption of the prenylation of these various targets plays an important role in statin-induced myopathy (Blanco-Colio et al. 2002; Matzno et al. 2005; Sakamoto et al. 2011).

\subsubsection{Lamins}

Lamins are intermediate filament proteins that are the main structural component of the nuclear lamina, adjacent to the inner nuclear membrane. In addition to its structural role, the nuclear lamina has important roles in chromatin organisation, cell division and gene transcription (Davies et al. 2009), and lamins are thought to also mediate the targeting of integral membrane proteins to the inner nuclear membrane (Wilson 2000). The importance of functional lamins for proper muscle function is emphasised by the fact that mutations in the gene coding for lamins $A$ and $C$ are known to cause a variety of muscle disorders, including Emery-Dreifuss muscular dystrophy (Wilson 2000).

Lamins are substrates of FT (Lane and Beese 2006) and they undergo posttranslational modification by farnesylation, which is thought to be important for the targeting of the lamins to the inner nuclear membrane (Davies et al. 2009). Lamins B1 and B2 are farnesylated (Farnsworth et al. 1989; Rusinol and Sinensky 2006) and prelamin $A$, a precursor to lamin $A$, undergoes farnesylation-dependent processing to form the functional protein (Davies et al. 2009). It has been shown in vitro that treatment with lovastatin inhibits processing of prelamin A (Beck et al. 1990), results in nuclear accumulation of prelamin A (Sinensky et al. 1994) and prevents the assembly of lamin A into the nuclear lamina (Lutz et al. 1992). It is unclear how disruption of lamin function could lead to myopathy, but it is possible that resulting disruption of nuclear stability could make muscle cells less able to 
sustain the forces from muscle contraction and more susceptible to apoptosis (Lammerding et al. 2004).

\subsubsection{Small G Proteins}

Small G-proteins, or small GTPases (guanine nucleotide-binding proteins), are monomeric proteins that cycle between an inactive guanosine diphosphate (GDP) bound state and an active guanosine triphosphate (GTP) bound state and have important roles in cellular signal transduction. Small G-proteins are a major target for prenylation. There are known to be over 100 small G-proteins in eukaryotes, divided into superfamilies and subfamilies. The most well studied of the small Gproteins are those in the Ras superfamily, including the Ras, Rho and Rab subfamilies (reviewed by (McTaggart 2006)).

\section{i] Ras small GTPases}

Ras small $G$ proteins have key roles in many signalling cascades, transducing receptor activation to effects on gene transcription and cellular proliferation. The best known of these is the mitogen-activated protein (MAP) kinase cascade. In this cascade, upon activation of a tyrosine kinase receptor Ras becomes activated and can bind to Raf proteins. These in turn phosphorylate and activate MEK (mitogen-activated protein kinase kinase), which then phosphorylates and activates MAP kinase (MAPK). MAPK then translocates to the nucleus where it activates various transcription factors (Matozaki et al. 2000; Vojtek and Der 1998).

Ras proteins are further substrates of FT (Casey and Seabra 1996), with the exception of K-RasB which can undergo geranylgeranylation by GGT-I in the presence of FT inhibitors (Whyte et al. 1997). The farnesylation of Ras is vital for its membrane localisation, and therefore also for its activation and function in downstream signal transduction (Dirks and Jones 2006). In vitro experiments have shown a reduction in Ras farnesylation in mouse myoblasts on simvastatin treatment, which could be rescued by co-incubation with farnesol (Mullen et al. 2010), and in rat skeletal myoblasts treated with a range of statins membrane- 
bound Ras was activated and detached, leading to cell death by apoptosis (Matzno et al. 2005).

\section{ii] Rho Subfamily GTPases}

Members of the Rho subfamily, such as Rho, Rac and cdc42, are involved in regulating cytoskeleton organisation. Their roles include regulating the formation of actin stress fibres and focal adhesions, as well as regulating microtubule stability. They can also have effects on gene expression (Fukata et al. 2003; Matozaki et al. 2000). Rho family proteins are substrates of GGT-I and usually undergo geranylgeranylation. Exceptions to this include proposed prenylation by FT, in addition to GGT-I, when these proteins are overproduced (Casey and Seabra 1996) and RhoB, which can be either farnesylated or geranylgeranylated by GGT-I (Armstrong et al. 1995).

There is varying evidence for the importance of disrupted prenylation of Rho proteins in statin-induced myopathy. In vitro treatment with statins leads to fragmentation of actin and collapse of the microtubule network (Bifulco et al. 1993; Liao 2002; Matzno et al. 1997) which can be reversed by mevalonate treatment (Bifulco et al. 1993; Liao 2002). In rat primary neurons, statin treatment has been shown to cause a decrease in membrane-bound Rho $A$ as well as disruption of the microtubule network (Meske et al. 2003). Simvastatin and fluvastatin treatment similarly led to a change in localisation of Rho A from the membrane to the cytosol and apoptosis in rat myoblasts, which could be rescued on co-addition of GGPP. A Rho-kinase inhibitor mimicked the statin-induced apoptosis and a similar change in Rho A localisation was observed in rat skeletal muscle in vivo (Itagaki et al. 2009). It has also been reported that myopathy resulting from treatment with colchicine, a drug that disrupts microtubule dynamics, results in similar pathology to statin-induced myopathy (Baker et al. 2004).

Moreover, statins have been shown to induce apoptosis in vitro in a range of different cells (Dirks and Jones 2006). One study has provided evidence that treatment of vascular smooth muscle cells with statins in vitro leads to a 
downregulation of the anti-apoptotic protein $\mathrm{Bcl}-2$ (B-cell lymphoma 2), via inhibition of the prenylation of Rho $A$, and that the resulting apoptosis can be rescued by addition of mevalonate, FPP or GGPP (Blanco-Colio et al. 2002). However, in contrast to this, another study found levels of membrane-bound Rho to be unaffected by statin treatment in rat myoblasts. The authors therefore suggested that statin-induced apoptosis of skeletal muscle is not caused directly by depletion of Rho, and they proposed that depletion of Rho may only be important in mediating the pleiotropic effects of statins in other tissues (Matzno et al. 2005).

\section{iii] Rab Subfamily GTPases}

Small G-proteins in the Rab subfamily are important in organelle biogenesis and intracellular trafficking of vesicles. There are numerous different isoforms, each of which has a different intracellular localisation and regulates a different trafficking step. Rab proteins are geranylgeranylated by GGT-II, which is an important modification for Rab protein localisation and therefore function (Leung et al. 2006).

Recent studies have demonstrated that inactivation of Rab proteins could play a crucial role in statin-induced morphological changes in muscle. Treatment with perillyl alcohol, an inhibitor of GGT-I and GGT-II, has been shown to mimic the effect of statins in isolated rat skeletal myofibres by inducing vacuolisation, degeneration and swelling of organelles. These effects could be rescued by coaddition of GGPP but not FPP, and could not be reproduced by specific inhibition of GGT-I or FT (Sakamoto et al. 2007). More recently, specific small molecule inhibition of GGT-II has been shown to produce similar effects to simvastatin treatment in mouse myotubes, in lowering the levels of cellular ATP (Wagner et al. 2011), whilst fluvastatin has been shown to inhibit translocation of Rab1A to the membrane in rat skeletal myofibres, an effect which could be rescued on cotreatment with GGPP. Treatment with Brefeldin A, a specific suppressor of the trafficking step regulated by Rab1A, reproduced the effects of vacuolation and myofibre cell death seen with fluvastatin treatment (Sakamoto et al. 2011). 
Therefore, although there is debate over which of the small G-proteins are the most important, (Matzno et al. 2005; Sakamoto et al. 2007) it does seem very likely that reduced prenylation plays an important role in statin-induced myopathy, most likely through the resulting disruption of muscle fibre stability, apoptosis of muscle fibres and defects in myoregeneration. However, the majority of studies described here only examine the effects of statins on prenylation in muscle, as a site of statin toxicity, and do not compare this to effects in the liver, as the target organ of the statins. They also tend to only use one or a small number of statins.

\subsubsection{Pleiotropic Effects of Statins}

Although reductions in the other isoprenoids of the mevalonate pathway may be the main cause of statin-induced myopathy it is thought that these effects, particularly those effects on the reduced prenylation of small $G$ proteins (Liao and Laufs 2005), may also contribute to the variety of pleiotropic effects observed. These pleiotropic effects of the statins are independent of cholesterol lowering and include reduced inflammation (Liang et al. 2006), decreased oxidative stress (Makabe et al. 2010; Schupp et al. 2008), improved endothelial function (Jacobson 2008), vasorelaxant effects (Yaktubay Dondas et al. 2011), anti-thrombotic effects (Jacobson 2008; Mason 2006) and effects on improving stability of atherosclerotic plaques (Abela et al. 2011; Mason 2006).

Therefore, although the statins are used primarily as lipid lowering drugs, there has been evidence indicating that they may also prove useful for treating conditions other than hypercholesterolaemia. There has been interest in the use of statins as anti-cancer agents, to be used in combination with chemotherapy or radiation. This is because statins have been shown to have anti-proliferative effects, to inhibit pathways associated with the invasion and metastasis of cancer cells and also to induce apoptosis in a variety of malignant tumour cells, including acute myelogenous leukaemia cells, possibly through decreased geranylgeranylation of proteins. Whereas apoptosis of healthy muscle fibres leading to myopathy is an unwanted side-effect, the apoptosis of cells in certain diseased tissue may prove 
beneficial for treating cancers. These anti-tumour effects have been demonstrated both in vitro and in vivo in animal models (Chan et al. 2003; Xia et al. 2001). Statins could be used to help overcome multidrug resistance, a significant problem during cancer treatment, through their inhibition of efflux transporters (Sieczkowski et al. 2010). It has also been suggested that statins may prove useful as a treatment for numerous other medical conditions, including asthma (Chiba et al. 2010), multiple sclerosis (Kuipers et al. 2006), their use may lead to a decreased risk of Alzheimer's disease (Cordle et al. 2005; Ostrowski et al. 2007) and they may be beneficial for patients with heart failure (Gullestad et al. 2007).

In summary, although the use of statins can be greatly beneficial, the reality of muscle toxicity continues to represent a problem in terms of both patient health and compliance. Therefore, it is important to fully understand the mechanism of this toxicity in order to find ways to reduce or avoid it whilst maintaining therapeutic doses. Should a patient experience myopathy, this is often managed by switching them either to a different statin or to a lower dose of the same statin (Harper and Jacobson 2010). However, as previously mentioned in section 1.2.2, there may also be potential for simply managing the problem of myopathy temporally, by attempting to time treatment with respect to circadian biology. 


\subsection{Circadian Rhythms}

Daily rhythms are known to regulate a multitude of biological processes including the daily sleep-wake cycle, body temperature, feeding, hormone secretion, glucose homeostasis, cell-cycle progression and metabolism (Takahashi et al. 2008). These circadian rhythms are endogenously generated, persist in the absence of external cues and have a period of approximately 24 hours (circa $=$ about; Diem = a day) (Hirota and Fukada 2004). They are beneficial to organisms as they allow their behaviour to be anticipatory of and adaptive to regular and predictable daily environmental changes (Wu et al. 2010). The ubiquitous nature of this regulation also has implications for the efficacy and toxicity of xenobiotics (Baraldo 2008; Levi and Schibler 2007).

\subsubsection{Chronotherapy}

Chronotherapy is the name given to the optimising of treatment by taking into account the temporal changes in drug pharmacokinetics and pharmacodynamics, as well as in the condition being treated, usually with the result of increased efficacy and/or reduced toxicity (Levi and Schibler 2007). Its importance in clinical practise is gradually being acknowledged (Baraldo 2008; Zmrzljak and Rozman 2012), and there are several cases where changing the timing of treatment is already making a difference in terms of both efficacy and toxicity (Levi and Schibler 2007). One such example is the decreased toxicity when changing to evening dosing of the NSAIDS (Non-steroidal anti-inflammatory drugs) indomethacin and ketoprofen in patients with hip or knee osteoarthritis. This is thought to be due to 24-hour variations in both the pharmacokinetics of these drugs and in joint pain. Greater efficacy and reduced toxicity has also been observed in response to colorectal cancer treatment administered using a chronomodulated time schedule as opposed to a constant rate infusion (Levi and Schibler 2007; Takahashi et al. 2008). In addition, there are numerous examples of where the potential for chronotherapy is currently being investigated, including treatment for asthma (Ohdo 2010), for hypertension (Portaluppi and Smolensky 2010) and treatment 
with a variety of chemotherapeutic agents (Innominato et al. 2010; Ohdo 2010; Shukla et al. 2011).

The case of statins could potentially be another example of where changing the time of dosing may prove beneficial. It is known that in humans cholesterol synthesis peaks in the night/early morning (midnight - 4:00 am) (Gälman et al. 2005; Jones and Schoeller 1990; Parker et al. 1982) and so it is thought that for maximum LDL-lowering efficacy it is best for statins with short half-lives (simvastatin, lovastatin, fluvastatin, cerivastatin) to be taken in the evening, but that the statins with longer half-lives, such as atorvastatin and rosuvastatin, can be taken in either the morning or evening (Martin et al. 2002; Muck et al. 2000; Plakogiannis and Cohen 2007; Plakogiannis et al. 2005). However, most of these studies looking into the effect of temporal dosing of statins focus primarily on temporal changes in their LDL-lowering efficacy, and do not also examine temporal changes in statin related muscle toxicity. It is therefore important to fully understand circadian changes that occur at the molecular level and how these changes might relate to statin treatment and the mevalonate pathway. 


\subsubsection{The Molecular Basis of Mammalian Circadian Rhythms}

Molecular clocks are responsible for the generation of circadian rhythms, where the molecular mechanism underlying oscillations within the molecular clock is thought to involve many transcriptional - translational feedback loops (Figure 1-5). Key in these feedback loops are the basic helix-loop-helix-PAS transcription factors BMAL1 (brain and muscle arnt-like protein-1) and CLOCK (circadian locomotor output cycles kaput) (Takahashi et al. 2008).

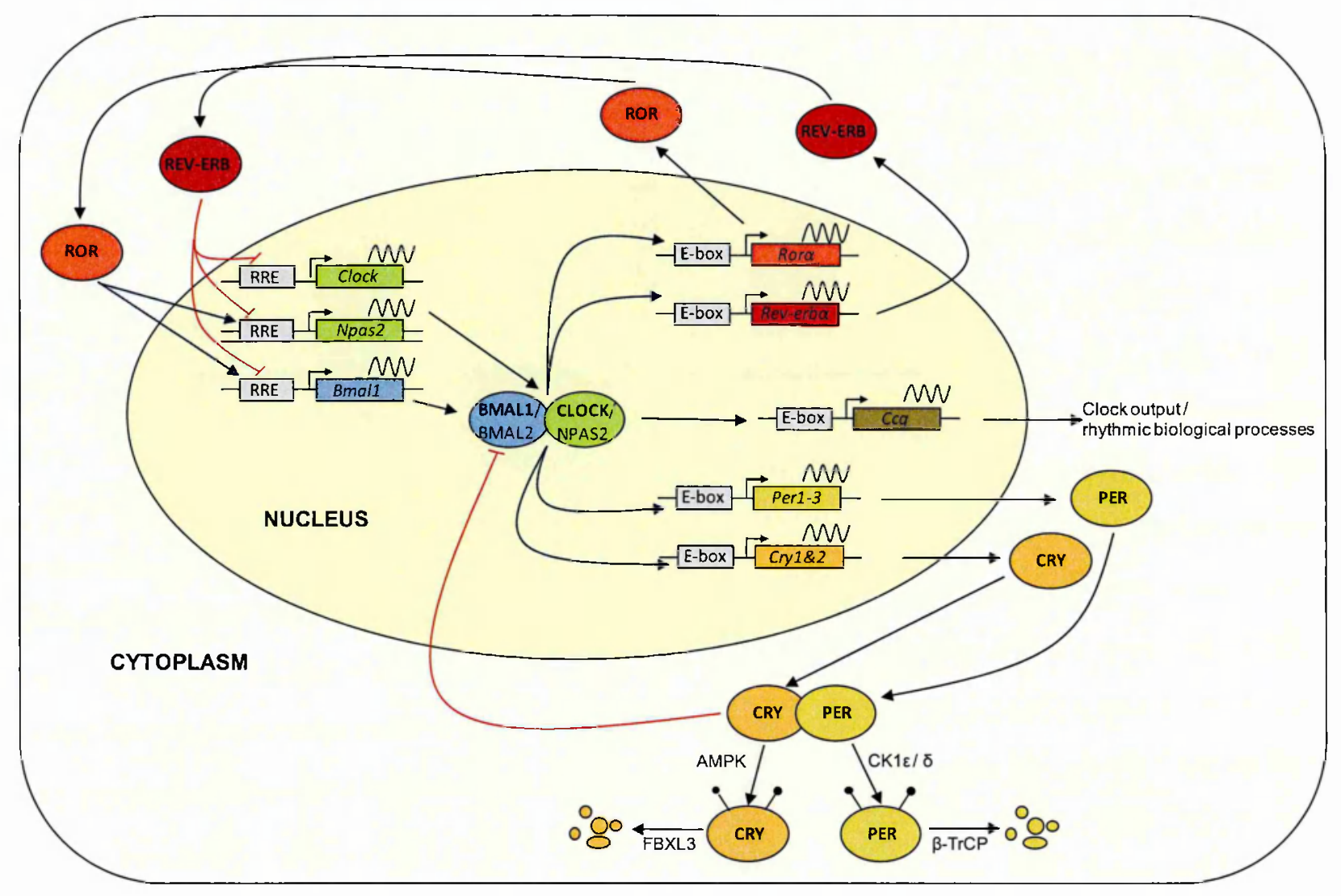

Figure 1-5 The mammalian molecular oscillator

In a network of transcriptional - translational feedback loops the BMAL1: CLOCK heterodimer activates transcription of core clock genes Per, Cry, Rev-erba, and Rora by binding to E-box elements. These gene products then activate or repress their own transcription through effects on BMAL1 and/or CLOCK (RRE = retinoic acid-related orphan receptor response element). BMAL2 and NPAS2 are alternative dimerisation partners for CLOCK and BMAL1 respectively. Post-translational modifications, such as phosphorylation by $C K 1 \delta, C K 1 \varepsilon$ and AMPK and degradation mediated by SCF ubiquitin E3 ligases FBXL3 and $\beta$-TrCP, ensure that the cycle has a period of approximately 24 hours. The core clock machinery also regulates the expression of many other clock-controlled genes $(\mathrm{Ccg})$ which have roles in numerous biological processes. Figure produced using (Crumbley and Burris 2011; Crumbley et al. 2010; Ko and Takahashi 2006; Kohsaka and Bass 2007; Lamia et al. 2009; Shi et al. 2010; Virshup and Forger 2007). 
Heterodimeric complexes of BMAL1 and CLOCK (BMAL1: CLOCK) activate transcription of the genes encoding period homologues (Per 1-3) and cryptochrome (Cry1 and Cry2) via binding to E-box elements (CACGTG) within enhancer and promoter sequences (Albrecht 2012). As PER and CRY proteins accumulate, they form protein complexes and translocate back to the nucleus where they repress their own transcription by interacting with the BMAL1: CLOCK complex (PER/CRY loop, bottom of Figure 1-5). The resulting decrease in PER and CRY protein levels, then allows BMAL1 and CLOCK to activate transcription of Per and Cry genes again, and the cycle continues (Levi and Schibler 2007). NPAS2 (neuronal PAS domain protein 2) has been reported to also dimerise with BMAL1 to form an active transcriptional complex in the absence of CLOCK (DeBruyne et al. 2006; DeBruyne et al. 2007), and likewise BMAL2 can act as an alternative dimerisation partner for CLOCK (Sasaki et al. 2009; Shi et al. 2010).

BMAL1: CLOCK also activates expression of the transcription factors Rora (retinoic acid-related orphan nuclear receptor $\alpha$ ) and Rev-erb $\alpha$ (reverse erythroblastosis virus $\alpha$ ) (Ko and Takahashi 2006). ROR $\alpha$ and REV-ERBa then compete to bind to retinoic acid-related orphan receptor response elements (RREs) in the promoters of Bmal1 (Preitner et al. 2002) and Npas2 (Crumbley et al. 2010), whereupon ROR $\alpha$ activates transcription and REV-ERBa represses transcription (ROR/REVERB loop, top of Figure 1-5). REV-ERBa has also been shown to regulate the expression of Clock (Crumbley and Burris 2011) and to interact with PER2 thereby coordinating these two feedback loops (Schmutz et al. 2010).

In addition, there are a number of other important feedback mechanisms. DBP (D site of albumin promoter (albumin D-box) binding protein) and E4BP4 are members of the PAR (proline and acidic amino acid-rich) basic leucine zipper transcription factor family. They antagonistically compete for D-box elements (Mitsui et al. 2001) and regulate expression of Per1 (Yamaguchi et al. 2000) as well as many clock controlled genes (ccg) (Noshiro et al. 2007). Basic helix-loop-helix transcription factors DEC1 (deleted in oesophageal Cancer 1 ) and DEC2 have been suggested 
to act as negative feedback regulators, as Dec1 expression is controlled by BMAL1:CLOCK, and DEC1 and DEC2 inhibit BMAL1:CLOCK function (Hirota and Fukada 2004; Honma et al. 2002)

Post-translational modifications, such as phosphorylation and ubiquitination, modify the activity and stability of clock proteins to ensure that a complete cycle takes approximately 24 hours (Hirota and Fukada 2004). The phosphorylation of PER proteins by casein kinase 1 epsilon and casein kinase 1 delta (CK1 $1 \varepsilon$ and $\mathrm{CK} 1 \delta$ ) (Akashi et al. 2002; Lowrey et al. 2000; Xu et al. 2005) has been shown to regulate both their nuclear translocation (Akashi et al. 2002; Takano et al. 2004; Vielhaber et al. 2000) and their stability (Meng et al. 2008). Degradation is determined by subsequent recognition of phosphorylated PER proteins by the Skp1-Cul1-F-Box protein (SCF) ubiquitin E3 ligases $\beta$-transducin repeat containing protein 1 ( $\beta$ TrCP1) and $\beta-T r C P 2$ (Ohsaki et al. 2008; Reischl et al. 2007; Shirogane et al. 2005). Stability of the CRY proteins is regulated through phosphorylation by adenosine monophosphate-activated protein kinase (AMPK) (Lamia et al. 2009) and by the SCF ubiquitin E3 ligase F-box and leucine-rich repeat protein 3 (FBXL3) (Busino et al. 2007; Godinho et al. 2007; Siepka et al. 2007) (Figure 1-5). It has been suggested that CRY and BMAL1 may also be substrates of CK1 $\varepsilon$ (Eide et al. 2002).

\subsubsection{Hierarchy of the Mammalian Circadian Timing System}

A master clock resides in the suprachiasmatic nucleus (SCN) within the anterior hypothalamus in the brain (Ko and Takahashi 2006). This clock is synchronised with the external 24-hour day via a number of environmental cues, or zeitgebers, the most important of which is thought to be light (Dibner et al. 2010; Hirota and Fukada 2004).

However, it has also been shown that there are molecular clocks in many peripheral tissues that regulate local rhythms (Yamazaki et al. 2000; Yoo et al. 2004), including in the liver (Oishi et al. 2005), heart (Leibetseder et al. 2009), 
adipose tissue (Zvonic et al. 2006), the vasculature (Reilly et al. 2007) and skeletal muscle (Zhang et al. 2009). These peripheral clocks are thought to be selfsustaining (Tahara et al. 2012; Yoo et al. 2004) but entrained both by neural and humoral signals originating from the master clock in the SCN (Hirota and Fukada 2004; Yamazaki et al. 2000) as well as by feeding (Damiola et al. 2000; Yamajuku et al. 2012). In vitro a serum pulse is able to synchronise the endogenous rhythms of a population of cells in culture (Balsalobre et al. 1998) and to date circadian rhythmicity has been observed in a wide variety of cell types (Balsalobre et al. 1998; Chalmers et al. 2008; Huang et al. 2009; Lemos et al. 2007; Li et al. 2008; Maronde and Motzkus 2003; Otway et al. 2009; Takiguchi et al. 2007).

The output of the clock is driven by the expression of many clock controlled genes (ccg), which show 24 hour cycles in their expression but are not themselves part of the core clock mechanism (Bozek et al. 2009). Circadian cycling has been shown to occur at both the transcript level, where studies have indicated that approximately $10 \%$ of genes in a variety of tissues are transcribed in a circadian manner (Akhtar et al. 2002; Panda et al. 2002; Storch et al. 2002), and the protein level, where $20 \%$ of all soluble proteins in mouse liver have been found subject to circadian control (Reddy et al. 2006). Circadian regulation has been shown to have tissue specific differences (Panda et al. 2002; Storch et al. 2002) and to include genes from most functional categories, including metabolism (McCarthy et al. 2007). 


\subsubsection{Circadian Control of Cholesterol Metabolism}

Many of the genes encoding enzymes and other proteins that are involved in the regulation of cholesterol metabolism have been shown to exhibit rhythmic patterns of expression (Figure 1-6).

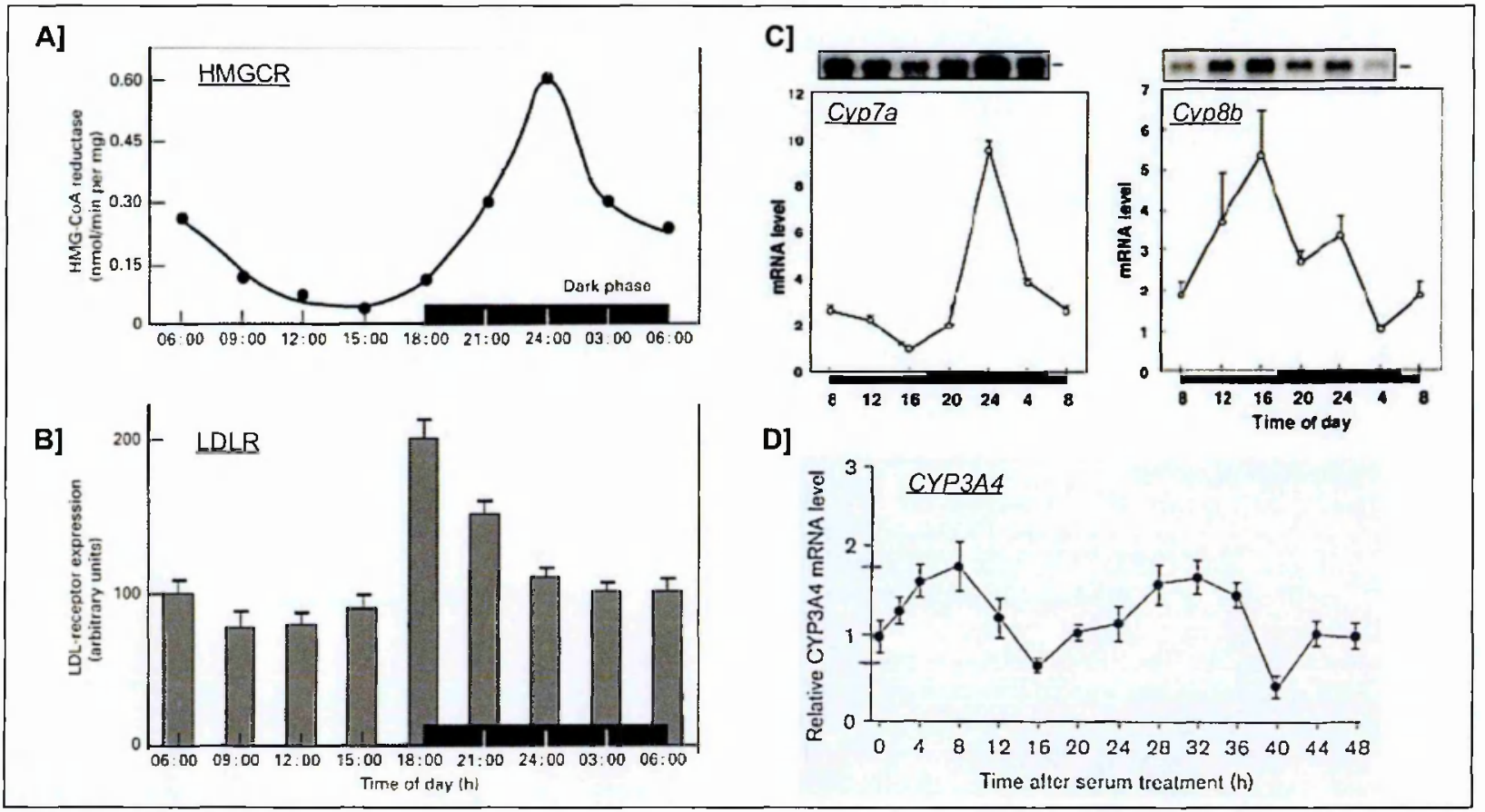

Figure 1-6 Daily rhythmicity in key enzymes and receptors involved in the regulation of cholesterol levels or statin metabolism

(A) 3-Hydroxy-3-methylglutaryl coenzyme A reductase (HMGCR) activity in rat liver (Balasubramaniam et al. 1994), (B) low-density lipoprotein receptor (LDLR) expression in rat liver (Balasubramaniam et al. 1994), (C) Cyp7a and Cyp8b mRNA levels, as measured in rat liver by northern blots (upper panels) and quantitative real time RT-PCR (lower graphs) (Noshiro et al. 2004) and (D) CYP3A4 mRNA levels in HepG2 liver cells (Takiguchi et al. 2007).

HMGCR has been shown to exhibit diurnal variation (Panda et al. 2002). Experiments in mouse liver have shown approximately a 2-fold difference in $\mathrm{Hmgcr}$ transcript levels between peak and nadir (Oishi et al. 2005) and in rat liver a 6-fold difference in activity between peak and nadir has been reported (Figure 1-6A) (Balasubramaniam et al. 1994). It has also been shown that LDLR expression exhibits diurnal variation (Figure 1-6B) that is independent of cellular cholesterol 
levels, where in rat liver LDLR expression has shown a 2-3 fold difference between peak and nadir (Balasubramaniam et al. 1994).

Cholesterol-7 $\alpha$-hydroxylase (CYP7A1) and sterol-12 $\alpha$-hydroxylase (CYP8B1) are two enzymes that are important for the conversion of cholesterol into bile acids in the liver, which is the most important route for metabolism and excretion of cholesterol in mammals. Both of these enzymes have been shown to exhibit diurnal mRNA expression patterns (Figure 1-6C) in the livers of both mice and rats (Noshiro et al. 2004; Noshiro et al. 2007) and the expression of Cyp7a1 has been shown to be regulated by a number of transcription factors, most notably by DBP, DEC2 and REV-ERBa, many of which are directly regulated by the CLOCK:BMAL1 heterodimer (Noshiro et al. 2007) (section 1.4.2).

The circadian rhythmicity of cholesterol metabolism has been further highlighted by in vivo studies investigating the output of core clock transcription factors REVERBa (Le Martelot et al. 2009) and RORa (Kang et al. 2007). In mouse liver REVERBa was shown to modulate the activity of both the transcription factor SREBP, which regulates the expression of many genes involved in cholesterol metabolism including HMGCR and LDLR (Brown and Goldstein 1997), and of the nuclear receptor liver-X-receptor (LXR), which regulates Cyp7A1 expression (Le Martelot et al. 2009). ROR transcription factors were found to similarly regulate the expression of Cyp8b1 (Kang et al. 2007).

It is also of interest to consider that circadian rhythmicity has been observed in CYP3A4, the cytochrome P450 enzyme involved in metabolism of simvastatin, lovastatin, cerivastatin and atorvastatin (section 1.1.4). In synchronised HepG2 liver cells, mRNA levels (Figure 1-6D), protein levels and metabolic activity of CYP3A4 were observed to fluctuate rhymically with a period of about 24 hours; a 2-fold difference was observed in CYP3A4 transcript level between peak and nadir, whilst increases and decreases in protein expression seemed to correspond to similar oscillations in enzymatic activity, as measured by the $6 \beta$-hydroxylation of 
testosterone (Takiguchi et ai. 2007). In addition, the expression of CYP2C8, an enzyme also involved in the metabolism of cerivastatin, has been reported to be regulated by the core clock transcription factor RORa (Chen et al. 2009b).

Taking all of this into consideration, it therefore seems likely that other aspects of the mevalonate pathway, for example protein prenylation, may exhibit circadian rhythmicity in the same way as has been demonstrated for cholesterol metabolism.

\subsection{Initial Hypothesis and Objectives}

The initial hypothesis for this project was therefore that:

- Protein prenylation, postulated to play an important role in statin-induced myopathy, is under circadian variation as has already been shown for other aspects of the mevalonate pathway.

It may therefore prove possible to achieve greater separation between the cholesterol-lowering efficacy of statins and muscle toxicity by changing the time of treatment.

\section{Aims and Objectives:}

The initial aim was to use liver and muscle cells in culture as a model system for comparing the effects of temporal statin administration on both cholesterol and isoprenoid biosynthesis. This was to be achieved via the following objectives;

Objective 1: characterise RD muscle cells and their differentiation process

- Induce RD cell differentiation using serum deprivation

- Measure mRNA expression of key differentiation markers

- Confirm expression of core clock genes and key cholesterol metabolism genes 
Objective 2: verify the ability of HepG2 (liver) and RD (muscle) cells to establish and maintain a circadian cycle

- Synchronise cells using the serum pulse technique

- Measure mRNA expression of core clock genes and key cholesterol metabolism genes

- Confirm transcript measurements at the protein level through western blotting

Objective 3: assess whether circadian changes in gene expression translate to circadian changes in cellular function.

At key points in the circadian cycle measure:

- HMGCR activity

- cholesterol level

- level of isoprenoids (F-PP and GG-PP)

- prenylated proteins in bound and soluble cell fractions

Objective 4: examine the effect of statin treatment on outputs measured in objectives 2 and 3 .

Objective 5: combine all data using computer modelling software to determine whether greater separation can be achieved between efficacy and toxicity. 


\section{Materials and Methods}

\subsection{Materials}

Unless otherwise stated in Table 2-1, all chemicals and materials were purchased from Sigma-Aldrich (Dorset, UK) and were of molecular biology standard.

\begin{tabular}{|c|c|}
\hline Item & Supplier \\
\hline \multicolumn{2}{|c|}{ Cell Culture } \\
\hline RD cells and $3 T 3-L 1$ cells & $\begin{array}{l}\text { American Type Culture Collection } \\
\text { (Teddington, UK) }\end{array}$ \\
\hline HepG2 cells & $\begin{array}{l}\text { European Collection of Cell Cultures } \\
\text { (Salisbury, UK) }\end{array}$ \\
\hline Huh7 cells & Dr S. Hood, GlaxoSmithKline (Ware, UK) \\
\hline $\begin{array}{l}\text { Dulbecco's modified eagle medium } \\
\text { (DMEM), foetal bovine serum (FBS), } \\
\text { horse serum, penicillin, streptomycin, } \\
\text { non-essential amino acids, trypsin-EDTA }\end{array}$ & Invitrogen GIBCO (Paisley, UK) \\
\hline Phosphate buffered saline tablets & Oxoid (Basingstoke, UK) \\
\hline \multicolumn{2}{|c|}{ Xenobiotics } \\
\hline $\begin{array}{l}\text { Simvastatin (sodium salt), simvastatin } \\
\text { lovastatin (sodium salt), lovastatin } \\
\text { pravastatin, fluvastatin }\end{array}$ & Calbiochem, Merck (Nottingham UK) \\
\hline Atorvastatin, rosuvastatin & Molekula Limited (Dorset, UK) \\
\hline $\begin{array}{l}\text { Cerivastatin, simvastatin (sodium salt), } \\
\text { pravastatin }\end{array}$ & $\begin{array}{l}\text { Sequoia Research Products Limited } \\
\text { (Pangbourne, UK) }\end{array}$ \\
\hline $\begin{array}{l}\text { FTI-277 trifluoroacetate salt, GGTI-2133, } \\
\text { perillyl alcohol }\end{array}$ & Sigma-Aldrich (Dorset, UK) \\
\hline
\end{tabular}




\begin{tabular}{|c|c|}
\hline \multicolumn{2}{|c|}{ RNA Analysis } \\
\hline $\begin{array}{l}\text { DNase/RNase free tips and } \\
\text { Eppendorf tubes }\end{array}$ & Axygen (Union City, USA). \\
\hline Fastrak Pipette Tips (RTPCR) & Alpha Laboratories (Eastleigh, UK) \\
\hline NucleoSpin II RNA extraction kit & Macherey-Nagel (Düren, Germany) \\
\hline $\begin{array}{l}\text { RNase-free DNase I, } 10 \times \text { DNase buffer, } \\
\text { DNase stop solution, } \\
\text { human genomic DNA, } \\
\text { mouse genomic DNA }\end{array}$ & Promega (Southampton, UK) \\
\hline $\begin{array}{l}\text { RNAse-free water, dNTPs, random } \\
\text { hexamers, Superscript II, DTT, } \\
\text { RNase OUT }\end{array}$ & Invitrogen GIBCO (Paisley, UK) \\
\hline $\begin{array}{l}\text { White Thermo-fast } 96 \text { PCR detection } \\
\text { plates, ABsolute QPCR ROX mix, } \\
\text { ABsolute QPCR seal }\end{array}$ & Abgene (Epsom, UK) \\
\hline TaqMan primers and probes & MWG Eurofins (Ebersberg, Germany). \\
\hline \multicolumn{2}{|c|}{ Protein Anaiysis } \\
\hline $\begin{array}{l}\text { Complete, mini, EDTA-free protease } \\
\text { inhibitor cocktail tablets }\end{array}$ & Roche (Lewes, UK) \\
\hline $\begin{array}{l}\text { ProteoExtract Native Membrane Protein } \\
\text { Extraction Kit }\end{array}$ & Calbiochem, Merck (Nottingham, UK) \\
\hline Folin-Ciocalteau's phenol reagent & Sigma-Aldrich (Dorset, UK) \\
\hline $\begin{array}{l}\text { Bis-acrylamide stock solution ( } 2 \% \mathrm{w} / \mathrm{v}) \\
\text { Acrylamide stock solution }(40 \% \mathrm{w} / \mathrm{v})\end{array}$ & WWR (Lutterworth, UK) \\
\hline
\end{tabular}




\begin{tabular}{|l|l|}
\hline $\begin{array}{l}\text { Novex Sharp Protein Standard } \\
\text { PVDF Western Blotting Membrane, }\end{array}$ & Invitrogen (Paisley, UK) \\
$\begin{array}{l}\text { Primary and secondary antibodies } \\
\text { (unless otherwise stated) }\end{array}$ & $\begin{array}{l}\text { Santa Cruz Biotechnology (Heidelberg, } \\
\text { Germany) }\end{array}$ \\
$\begin{array}{l}\text { Myogenin antibody } \\
\text { Myosin heavy chain } 3 \text { antibody }\end{array}$ & $\begin{array}{l}\text { Developmental Studies Hybridoma Bank } \\
\text { (lowa City, USA) }\end{array}$ \\
$\begin{array}{ll}\text { B-actin antibody (mouse monoclonal) } \\
\text { Lactate dehydrogenase antibody (goat } \\
\text { polyclonal) }\end{array}$ & $\begin{array}{l}\text { Sigma-Aldrich (Dorset, UK) } \\
\text { Abcam (Cambridge, UK) }\end{array}$ \\
\hline $\begin{array}{l}\text { ECL Plus Western Blotting Detection } \\
\text { System }\end{array}$ & $\begin{array}{l}\text { Chvitrogen Molecular Probes } \\
\text { (Eugene, USA) }\end{array}$ \\
\hline $\begin{array}{l}\text { Amplex Red Cholesterol Assay Kit } \\
\text { Gealthcare (Chalfont St Giles, UK) }\end{array}$ \\
\hline
\end{tabular}

Table 2-1 Suppliers of specialist materials used in this work 


\subsection{Methods}

\subsubsection{Cell Culture of Immortalised Cell Lines and Cell-Based Procedures}

Cell culture materials were purchased from Invitrogen GIBCO, (Paisley, UK) unless otherwise stated.

\subsubsection{RD Cell Culture}

The human embryonal rhabdomyosarcoma (RD) cell line is a prototypic, immortalised cell line derived from embryonal rhabdomyosarcoma tissue (McAllister et al. 1969). It is used as an in vitro model of skeletal muscle as these cells express a number of muscle-specific proteins (Kobayashi et al. 2008). RD cells were routinely cultured in Dulbecco's modified eagle medium (DMEM) with phenol red containing 4.5g/L glucose and L-glutamine, supplemented with $10 \%$ foetal bovine serum (FBS), 100U/ml Penicillin and 100U/ml Streptomycin.

\subsubsection{Huh7 Cell Culture}

The human hepatoma cell line, Huh7, is a differentiated, immortalised liver cell line derived from a hepatocellular carcinoma. These cells produce a variety of plasma proteins and enzymes that would be expected in liver cells in vivo (Nakabayashi et al. 1982). Huh7 cells were cultured in DMEM with phenol red containing 4.5g/L Dglucose and L-glutamine, supplemented with $10 \%$ FBS, $1 \%$ non-essential amino acids (NEAA), 100U/ml Penicillin and 100U/ml Streptomycin.

\subsubsection{HepG2 Cell Culture}

The human hepatoma cell line, HepG2, is a differentiated, immortalised liver cell line derived from a hepatoblastoma (Aden et al. 1979; Knowles et al. 1980). HepG2 cells were cultured in DMEM with phenol red containing $4.5 \mathrm{~g} / \mathrm{L}$ D-glucose and L-glutamine, supplemented with 10\% FBS, 1\% non-essential amino acids (NEAA), 100U/ml Penicillin and 100U/ml Streptomycin. 


\subsubsection{3T3-L1 Cell Culture}

The 3T3-L1 cell line is a murine preadipocyte cell line (Mackall et al. 1976) that has frequently been used to study diabetes, obesity and other metabolic disorders (Carlotti et al. 2004). 3T3-L1 cells were cultured in DMEM with phenol red containing 4.5g/L D-glucose and L-glutamine, supplemented with $10 \% \mathrm{FBS}, 1 \%$ sodium pyruvate, $100 \mathrm{U} / \mathrm{ml}$ Penicillin and $100 \mathrm{U} / \mathrm{ml}$ Streptomycin.

\subsubsection{Sub-Culturing Cells}

Cells were passaged when they reached approximately $70-80 \%$ confluence. The medium was first aspirated from the flasks. Cells were then washed in sterile phosphate buffered saline (PBS) and $3-4 \mathrm{ml}$ trypsin-EDTA solution added (for a $75 \mathrm{~cm}^{2}$ vented tissue culture flask (T75)). After coating the base of the flask, most of the trypsin solution was aspirated off before leaving the flask at $37^{\circ} \mathrm{C}$ for approximately 5 minutes, or until cells had detached from the bottom of the flask. After cell detachment, cells were resuspended in fresh complete medium, to inactivate the trypsin-EDTA, and pipetted up and down, to break up any cell clumps. The cell suspension was then pipetted into a fresh T75 flask, containing complete medium, to give the required cell split ensuring that the total volume in the flask was $15 \mathrm{ml}$. The precise ratio for cell splitting varied between $1: 2$ and 1:10, depending on the cell type and the experiment being carried out. Cells were kept in an incubator at $37^{\circ} \mathrm{C}$ with $5 \% \mathrm{CO}_{2}$.

For a $25 \mathrm{~cm}^{2}$ vented tissue culture flask (T25), a final volume of $5 \mathrm{ml}$ in each flask was used and $1 \mathrm{ml}$ of trypsin solution was used to detach cells. In a 6-well plate, the final volume in each well was $2.5 \mathrm{ml}$. All 6-well and 96-well plates were kept in a humidified chamber in an incubator at $37^{\circ} \mathrm{C}$ with $5 \% \mathrm{CO}_{2}$. 


\subsubsection{Counting Cells using a Haemocytometer}

Cells were detached from the culture flask by trypsinisation (section 2.2.1.5) and resuspended in fresh complete medium. After moistening the edges, a cover slip was affixed to the haemocytometer by applying gentle pressure, and $8.5 \mu \mathrm{L}$ of cell suspension was pipette into each chamber. In each chamber, the cells in 3 different large squares were counted and an average taken. After taking any dilutions into account, this value represented the number of cells $\times 10^{4}$ per $\mathrm{ml}$ in the original cell suspension.

\subsubsection{Storage of Cells in Liquid Nitrogen}

For long term storage, cells at an early passage number were detached from the culture flask by trypsinisation (section 2.2.1.5) and counted using a haemocytometer (section 2.2.1.6). Cells were then pelleted by centrifugation at $500 \mathrm{~g}$ for 3 minutes and resuspended in sufficient freezing medium (90\% DMEM, $10 \%$ dimethyl sulphoxide, DMSO) to give $1 \times 10^{6}$ cells $/ \mathrm{ml}$. $1 \mathrm{ml}$ aliquots in cryovials were transferred to the NALGENE cryo freezing container for storage at $-80^{\circ} \mathrm{C}$ for 24 hours, to allow slow freezing, before storage in liquid nitrogen until further use.

\subsubsection{Recovery of Cells from Liquid Nitrogen}

A cryovial, containing $1 \mathrm{ml}$ of cell suspension, was thawed from liquid nitrogen in a $37^{\circ} \mathrm{C}$ water bath. Once thawed, the cell suspension was transferred to a sterile tube containing $9 \mathrm{ml}$ of pre-warmed complete medium and centrifuged at $125 \mathrm{~g}$ for 7 minutes. The supernatant was removed and the cell pellet resuspended in $8 \mathrm{ml}$ fresh pre-warmed complete medium, which was then placed in a T25 culture flask. Medium was changed the following day, to remove any remaining traces of DMSO and unattached cells. Once the cells were $70-80 \%$ confluent they were trypsinised and transferred to a T75 culture flask. 


\subsubsection{RD cell Differentiation}

RD cells were grown to confluence in T25 culture flasks, 6-well plates or 96-well plates and then induced to differentiate by changing the medium to low serum medium (1\% FBS; Figure 2-1). In a preliminary experiment, to characterise the differentiation process, cells were grown in T25 flasks and every 2 days cell photographs were taken and RNA extracted for analysis by quantitative RT-PCR (see section 2.2.2). After day 0 , low-serum medium was refreshed just before taking cell photographs, in all flasks other than those being used to extract RNA that day, and every day after day 4. Scale bar on the cell photographs was derived from comparison with the literature (Vachon et al. 1996).

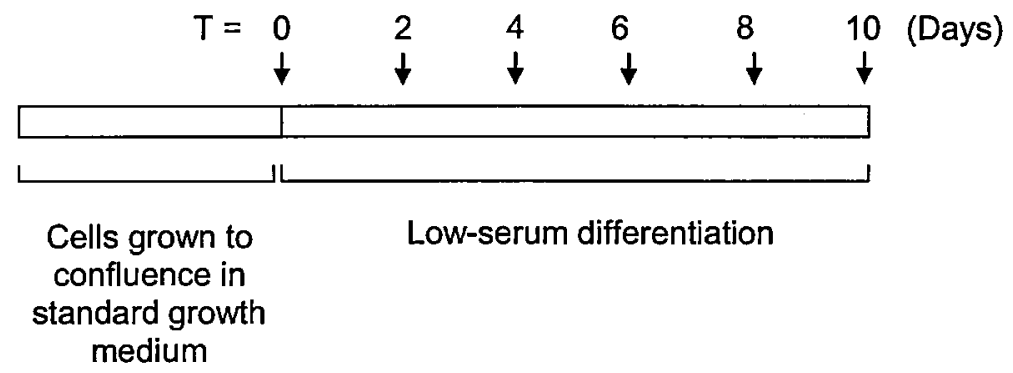

Figure 2-1 Protocol for differentiation of RD cells

RD cells were grown to confluence in standard growth medium (10\% FBS) and at $T=0$ medium was changed to low-serum medium (1\% FBS). At indicated 2-day intervals, cells were photographed and total RNA extracted for analysis by quantitative RT-PCR (TaqMan).

As a result of this analysis, it was decided that a period of 5 days in low-serum medium would be used for the differentiation of RD cells for future experiments. In the remainder of this thesis, the term "RD-D" is used to refer to RD cells that have undergone this 5-day differentiation protocol prior to use. "RD-U" refers to undifferentiated RD cells that have not undergone differentiation in low-serum medium prior to use. 


\subsubsection{Synchronising Cells using a Serum Pulse}

The serum pulse technique (Figure 2-2 and Figure 2-3) is a way of synchronising the molecular clocks in individual cells in a cell culture, using shock with a high concentration of serum, in order to study their circadian rhythms (Balsalobre et al. 1998).

In an initial pilot study 3T3-L1, HepG2 and Huh7 cells were seeded at densities of $2 \times 10^{5}, 1 \times 10^{5}$ and $2 \times 10^{5}$ cells per well respectively on 6-well plates, using 1 plate per time point per cell type. All cells were grown to 2 days post-confluence, after which the medium was changed to medium containing $50 \%$ horse serum $(T=0)$. After 2 hours medium was changed to serum-free medium, after which the medium was not changed again for the remainder of the experiment (Figure 2-2). RNA was extracted from 4 wells of 3T3-L1 and HepG2 cells at $T=0,14,26,36$ and from Huh7 cells at T=0,12, 18 and 26 and analysed by quantitative RT-PCR (TaqMan; section 2.2.2).
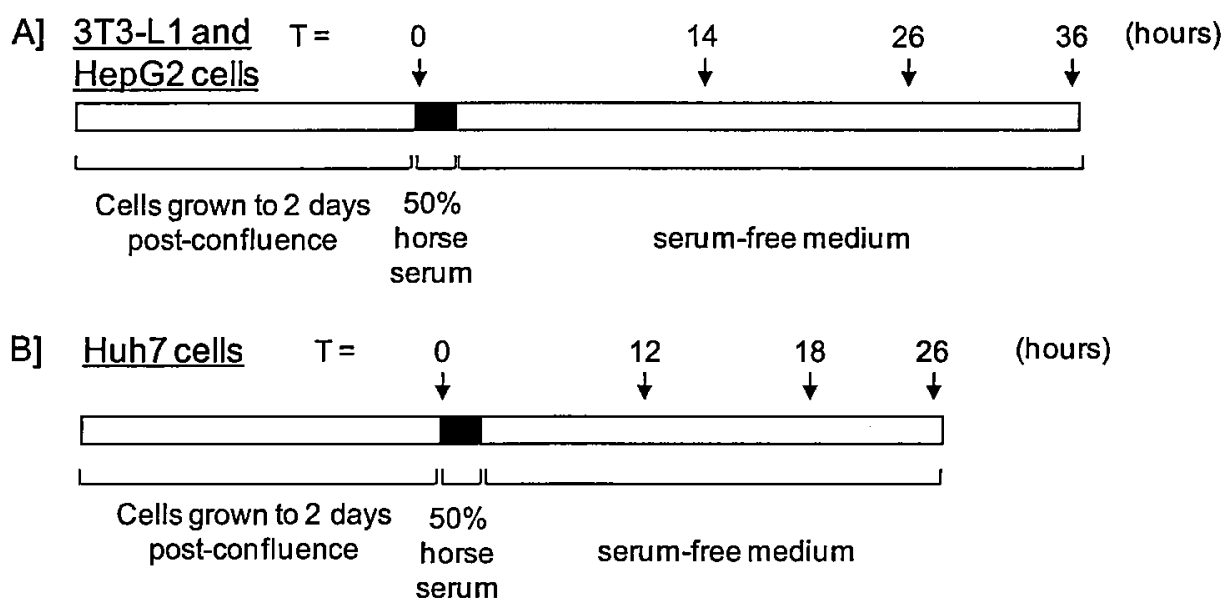

Figure 2-2 Protocol for pilot serum pulse experiment

At indicated intervals total RNA was extracted from A] 3T3-L1 and Huh7 cells, B] Huh7 cells for analysis by quantitative RT-PCR (TaqMan).

Subsequently RD and Huh7 cells were grown in 6-well plates, using 1 plate per time point per cell type: Huh7 cells were seeded at a density of $2 \times 10^{5}$ cells per well and grown to 2 days post-confluence. RD cells were seeded at a density of $3 x$ $10^{5}$ cells per well and used immediately following a 24 hour period for cell 
attachment (RD-U), and were seeded at $1 \times 10^{5}$ cells per well and allowed to differentiate for 5 days (RD-D; section 2.2.1.9). Medium was changed to medium containing $50 \%$ horse serum $(T=0)$. After 2 hours medium was changed to serumfree medium, after which the medium was not changed again for the remainder of the experiment (Figure 2-3). Every 4 hours starting from $T=0$, for each cell type, RNA was extracted from 4 wells for analysis by quantitative RT-PCR (TaqMan; section 2.2.2).

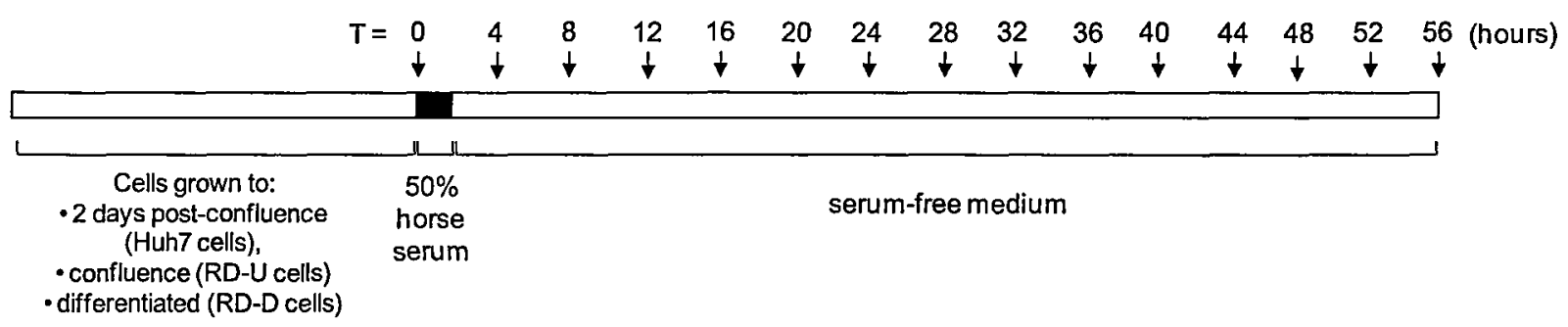

Figure 2-3 Protocol for serum pulse of Huh7 and RD cells

At indicated 4-hour intervals total RNA was extracted for analysis by quantitative RT-PCR (TaqMan).

\subsubsection{Treatment of Cells with Xenobiotics}

Cells were seeded as required in 96-well plates or 6-well plates (sections 2.2.1.10, $2.2 .1 .12,2.2 .3 .1,2.2 .4 .1$, with a final medium volume of $200 \mu \mathrm{l}$ in each well of a $96-$ well plate, $2.5 \mathrm{ml}$ in each well of a 6 -well plate or $5 \mathrm{ml}$ in each T25 flask. Seeding densities ranged from $2 \times 10^{3}-1 \times 10^{4}$ in 96-well plates and $1-4 \times 10^{5}$ in 6-well plates.

After 24 hours, medium was aspirated from each well and replaced with serum-free medium containing either xenobiotic or vehicle control (see Table 2-2), which was prepared as follows: Each xenobiotic was dissolved in a relevant solvent (see Table 2-2) to give a stock solution of $100 \mathrm{mM}$. This was then further diluted as needed, in the solvent, to give concentrations of xenobiotic that were 1000 -fold more concentrated than that required in the final medium. These stocks were then diluted in serum-free medium by 1:1000, in order to maintain the percentage of solvent in the final medium at $0.1 \%$. $100 \mu$ of xenobiotic/control-containing serumfree medium was added to each well of a $96-w e l l$ plate, or $2.5 \mathrm{ml}$ to each well of a 
6-well plate. Note that the sodium salts, of simvastatin and lovastatin, are referred to as their acid forms (in contrast to the lactone form) in subsequent chapters.

\begin{tabular}{|c|c|}
\hline Xenobiotic & Solvent \\
\hline Simvastatin (sodium salt) & DMSO \\
\hline Simvastatin & Ethanol \\
\hline Lovastatin (sodium salt) & Ethanol \\
\hline Lovastatin & DMSO \\
\hline Cerivastatin & Water \\
\hline Atorvastatin & DMSO \\
\hline Fluvastatin & Water \\
\hline Rosuvastatin & DMSO \\
\hline Pravastatin & Water \\
\hline FTI-277 & Water \\
\hline GGTI-2133 & DMSO \\
\hline Perillyl Alcohol & Water \\
\hline
\end{tabular}

\section{Table 2-2 Solvents used for xenoblotics}

Relevant solvent was also used as the related vehicle control.

\subsubsection{Assessing Cell Viability using the MTT Assay}

The 3-(4,5-dimethylthiazol-2-yl)2,5-diphenyl tetrazolium bromide (MTT) assay is a colorimetric assay that is used to quantitatively assess metabolic and cellular viability. The tetrazolium bromide (MTT) salt is reduced by dehydrogenase enzymes, in active mitochondria in living cells, to form a water-insoluble, coloured formazan salt. The amount of formazan generated, and absorbance measured, is directly proportional to the number of viable cells, over the range $200-50,000$ cells per well (Mosmann 1983).

In these experiments, RD cells were seeded at a density of $2 \times 10^{3}$ cells per well on 96-well plates and left to differentiate for 5 days in low-serum medium (RD-D; section 2.2.1.9). Huh7 and RD-U cells were seeded on 96-well plates 24 hours prior to experiment, at densities of $5 \times 10^{3}$ cells per well and $1 \times 10^{4}$ cells per well respectively. 
Cells were exposed to various statin concentrations or a vehicle control (see section 2.2.1.11) for 48 hours. $10 \mu \mathrm{l}$ of MTT solution $(5 \mathrm{mg} / \mathrm{ml}$ MTT dissolved in PBS and then filter sterilised) was added to each well for the final 2.5 hours of the 48 hour dosing period. Medium was then replaced with $100 \mu$ DMSO per well and the absorbance read at $540 \mathrm{~nm}$ in the Multiskan RC microplate reader (ThermoFisher Scientific, Loughborough, UK). Absorbance measured was expressed as a percentage of the vehicle control (defined as $100 \%$ ). At each statin concentration, mean absorbance values were calculated from each independent experiment, which were then combined to generate final data.

\subsubsection{RNA Analysis}

For RNA work, all tips and Eppendorf tubes used were DNase/RNase free. For TaqMan, non-filter tips and Eppendorf tubes were autoclaved prior to use.

\subsubsection{RNA Isolation}

Total RNA was extracted from 3T3-L1, HepG2, Huh7, RD-U and RD-D cells using the Nucleospin II RNA extraction kit according to the manufacturer's instructions: Cells were first lysed by the addition of the lysis buffer provided (RA1) and the viscosity reduced by passing each lysate through a 21 gauge needle several times. The lysate was then filtered using a provided NucleoSpin Filter, to further reduce viscosity and remove cellular debris. The flow-through from this column was loaded onto a NucleoSpin RNA II Column, in order to bind the RNA to the silica membrane, after the adjustment of the binding conditions with the addition of ethanol. Membrane desalting buffer was then added to the column, followed by a centrifugation step at $11,000 \mathrm{~g}$ for 1 minute to dry the membrane. Each column was washed with provided wash buffers RA2 and RA3, before the final elution of pure RNA in $60 \mu \mathrm{l}$ RNAse-free water.

Total RNA concentration was measured using a NanoDrop spectrophotometer (NanoDrop Technologies, Delaware, USA) using the A260/280 and A260/230 ratios as an indication of RNA quality. For pure RNA the expected $260 / 280$ ratio is 
in the region of 1.8-2.0 and the expected $260 / 230$ ratio is approximately 2.0. RNA absorbs light with an absorption peak at $260 \mathrm{~nm}$ and so if the $260 / 280$ ratio is much lower than this it is an indication that there may be protein contamination in the sample as protein absorbs light at $280 \mathrm{~nm}$. If the $260 / 230$ ratio is much lower, this may be an indication of sample contamination by for example thiocyanate ions, which are present in the buffers RA1, RA2 and MDB that are used in the RNA extraction (NanoDrop 2007).

RNA quality was also checked by running $1 \mu \mathrm{g}$ of each sample on a $1 \%$ agarose gel, containing ethidium bromide $(0.5 \mu \mathrm{g} / \mathrm{ml})$, in $1 \times T A E$ buffer $(0.04 \mathrm{M}$ Tris acetate, $0.01 \mathrm{M}$ EDTA). Samples were diluted in RNAse free water to $1 \mu \mathrm{g}$ in a total volume of $10 \mu \mathrm{l}$. This was then mixed with $2 \mu$ l Orange G loading dye $(0.25 \%$ Orange $G$, $50 \%$ glycerol) and the full $12 \mu$ loaded into the wells. Bands were visualised under UV light using the Gene Genius Bio Imaging System (Syngene, Cambridge, UK). If the RNA was intact and of good quality then sharp bands representing $28 \mathrm{~S}$ and $18 \mathrm{~S}$ ribosomal RNA (rRNA) were present on the resulting gel (Figure 2-4), with no obvious low molecular weight smear which would indicate degraded RNA (Applied Biosystems 2009). RNA was stored at $-80^{\circ} \mathrm{C}$.

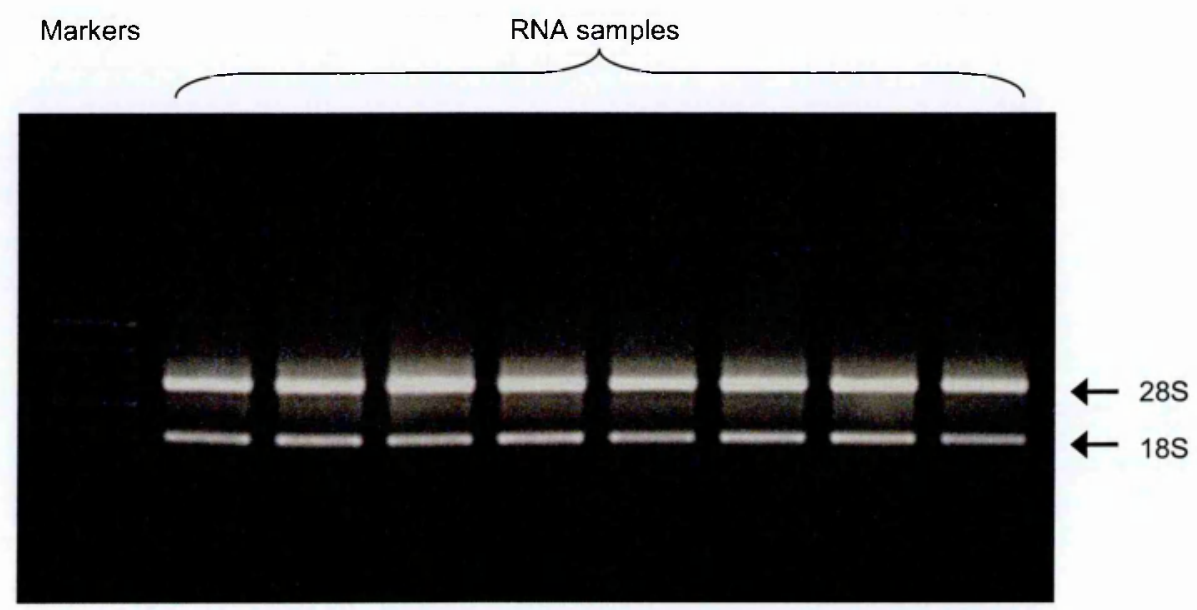

Figure 2-4 Example of how an agarose gel can be used to give an indication of RNA quality RNA samples shown here were extracted from RD muscle cells on days 6 and 8 of differentiation $(\mathrm{N}=4)$. Arrows indicate bands representing $28 \mathrm{~S}$ and $18 \mathrm{~S}$ rRNA. If RNA is intact these bands should be sharp with more $28 \mathrm{~S}$ present than $18 \mathrm{~S}$ (approximately twice as much $28 \mathrm{~S}$ ), as is shown here. 


\subsubsection{DNase Treatment}

All RNA samples were DNase treated to remove contaminating genomic DNA. $3 \mu \mathrm{g}$ total RNA, $2 \mu \mathrm{l} 10 \times$ DNase buffer and $2 \mu \mathrm{l}$ RNase-free DNase I were added to the volume of RNAse-free water required to bring the total reaction mixture to a total volume of $20 \mu \mathrm{l}$. This reaction mixture was incubated at $37^{\circ} \mathrm{C}$ for 30 minutes, followed by a heat inactivation step by the addition of $2 \mu \mathrm{l}$ DNase stop solution (20mM EGTA) and incubation at $65^{\circ} \mathrm{C}$ for 10 minutes.

\subsubsection{3 cDNA Synthesis}

$4 \mu$ l of the DNA-free RNA solution was added to $1.5 \mu \mathrm{l}$ dNTP mix (in which each dNTP was at a concentration of $10 \mathrm{mM}), 1.5 \mu$ random hexamers $(150 \mathrm{ng} / \mu \mathrm{l})$ and $5 \mu \mathrm{l}$ RNase-free water. This was incubated at $65^{\circ} \mathrm{C}$ for 5 minutes, to denature the RNA, and then cooled on ice for 2 minutes. $8 \mu$ l of a reverse transcriptase (RT+) mix was then added to each RNA/primer mix, where this RT+ mix contained $4 \mu \mathrm{l} 5 \mathrm{x}$ FS buffer (first strand synthesis buffer; supplied with Superscript II Reverse Transcriptase enzyme), $2 \mu \mathrm{l} 0.1 \mathrm{M} \mathrm{DTT}, 1 \mu \mathrm{l}$ RNase OUT, $0.75 \mu \mathrm{l}$ water and $0.25 \mu \mathrm{l}$ Superscript II for each reaction. This was then incubated at $25^{\circ} \mathrm{C}$ for 10 minutes, $42^{\circ} \mathrm{C}$ for 50 minutes and $70^{\circ} \mathrm{C}$ for 15 minutes. In a similar way, a reverse transcriptase negative (RT-) control was also prepared using each RNA sample, where the RNase OUT and Superscript II had been replaced by RNase-free water. All RT+ and RT- reaction mixes were then diluted by adding $80 \mu$ l RNase-free water and stored at $-20^{\circ} \mathrm{C}$.

\subsubsection{Quantitative Reverse Transcriptase Polymerase Chain Reaction}

$5 \mu$ of either RT + or RT- sample was pipetted into each well of a white Thermo-fast 96 PCR detection plate followed by $20 \mu \mathrm{l}$ of reaction mix; where this reaction mix was made up beforehand in bulk and contained $1 \mu$ forward and reverse primers (each primer at $10 \mathrm{pmol} / \mu \mathrm{l}), 0.5 \mu \mathrm{l}$ probe $(5 \mathrm{pmol} / \mu \mathrm{l}), 6 \mu \mathrm{l}$ sterile water and $12.5 \mu \mathrm{l}$ ABsolute QPCR ROX mix per reaction. Each plate was sealed with an ABsolute QPCR seal, briefly centrifuged and run on the ABI Prism 7000HT sequence detection system (Applied Biosystems, California, USA) using the cycle: 2 minutes 
at $50^{\circ} \mathrm{C}, 15$ minutes at $95^{\circ} \mathrm{C}, 40$ cycles of 15 seconds at $95^{\circ} \mathrm{C}$ and 1 minute at $60^{\circ} \mathrm{C}$. Relative transcript expression was calculated by comparison with a standard curve, made up of $10^{5}, 10^{4}, 10^{3}, 10^{2}$ and $10^{1}$ copies of human or mouse genomic DNA. For each primer/probe set used, duplicate no template controls (NTC) were also run, where $5 \mu$ l sterile water was used in place of RT+ or RTsample. Expression data were normalised, as indicated, to that of glyceraldehyde 3-phosphate dehydrogenase (GAPDH), 18 S ribosomal RNA (rRNA) or $\beta$-actin.

All gene-specific primers and probes used were designed using Vector NTI Advance 11 (Invitrogen) and Primer Express Version 2.0 (Applied Biosystems), and ordered from MWG Eurofins (Ebersberg, Germany). Primer and probe sequences are given in Table 2-3 and Table 2-4. Primer sets were designed to be within an exon as this meant that genomic DNA could be used for creating the standard curve. Genomic DNA is easy to work with and, provided that genomic DNA from the correct species is being used, it should contain all of the target genes in varying amounts, removing the need to create a different standard curve using CDNA for each target gene to be analysed. 


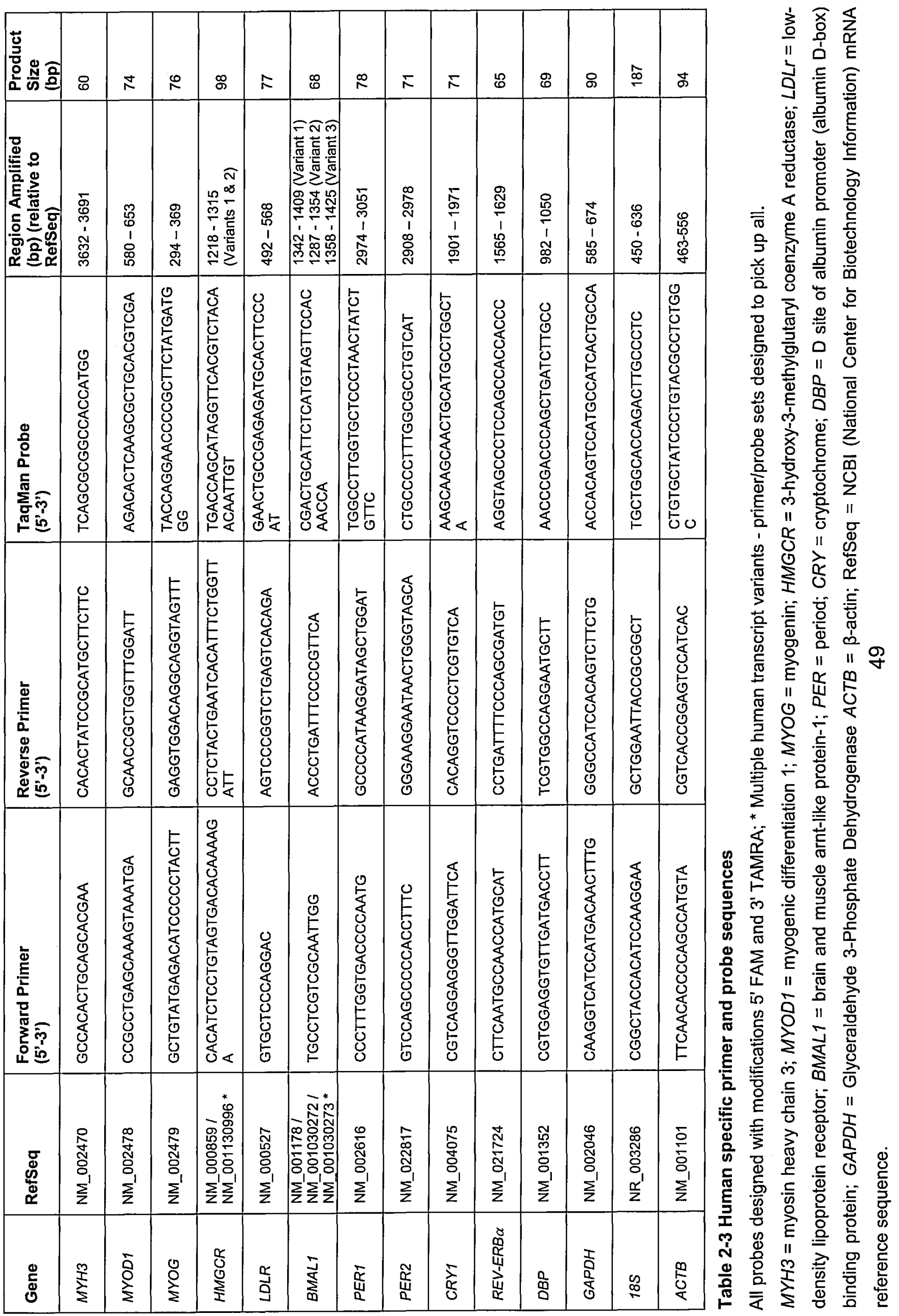




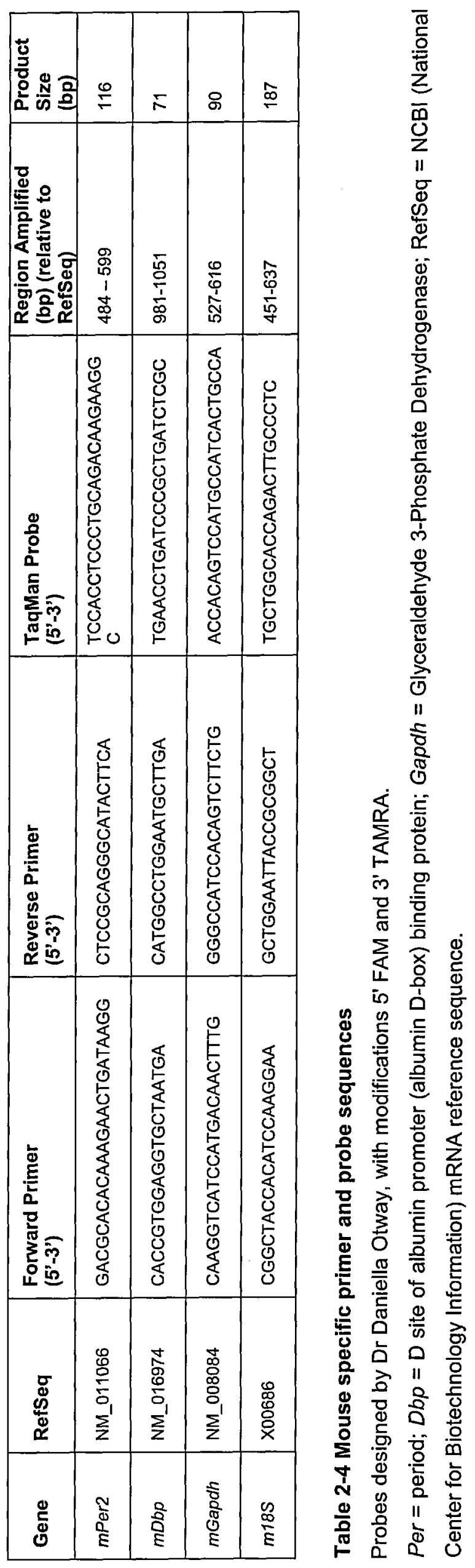




\subsubsection{Protein Analysis}

\subsubsection{Total Protein Extraction using RIPA Buffer}

HepG2, Huh7, RD-U and RD-D cells were grown in T25 flasks or 6-well plates and total protein was extracted from cells using RIPA buffer (RadiolmmunoPrecipitation Assay Buffer; $1 \%$ Nonidet-P40, $0.5 \%$ sodium deoxycholate, $0.1 \%$ sodium dodecyl sulphate (SDS) in $1 \times$ PBS, with 1 complete, mini, EDTA-free protease inhibitor cocktail tablet per $10 \mathrm{ml}$ buffer). Medium was aspirated off cells and cells were washed twice with PBS before the addition of ice cold RIPA lysis buffer ( $1 \mathrm{ml}$ per T25 flask or $300 \mu \mathrm{l}$ per well of a 6-well plate). Flasks or plates were left on ice for 30 minutes to allow cell lysis. After this time, lysate was homogenised and the DNA sheared using a 25 gauge needle, before being centrifuged for 15 minutes at $15,000 \mathrm{~g}$ at $4^{0} \mathrm{C}$. The supernatant from this step contained the total cell protein, which was stored at $20^{\circ} \mathrm{C}$ for regular use or at $-80^{\circ} \mathrm{C}$ for longer term storage. Each sample was used a maximum of 3 times, to minimise the effects of freeze-thawing. Samples to be used more frequently than this were frozen in aliquots.

\subsubsection{Membrane Protein Extraction}

Huh7, RD-U and RD-D cells were grown in 6-well plates and membrane protein extracted using the ProteoExtract Native Membrane Protein Extraction Kit. Medium was aspirated off the cells and cells were washed twice using $0.8 \mathrm{ml}$ ice cold wash buffer (provided in the kit). $4 \mu$ l Protease Inhibitor Cocktail was added to the wall of each well followed by $0.8 \mathrm{ml}$ Extraction Buffer I. Plate was swirled, to ensure mixing and covering of the cells, before incubation on ice for 10 minutes under gentle agitation. After incubation, supernatant enriched in cytosolic proteins was removed and stored at $-20^{\circ} \mathrm{C}$ until use. $2 \mu$ l Protease Inhibitor Cocktail and $0.4 \mathrm{ml}$ Extraction Buffer II was then similarly added to each well, and plates incubated on ice for 30 minutes under gentle agitation. Supernatant, enriched in integral membrane proteins and membrane associated proteins, was then removed and stored at $-20^{\circ} \mathrm{C}$ until use. If cells started to detach from the plate after any wash steps or incubation steps, cell suspension was removed, centrifuged for 15 minutes at $15,000 \mathrm{~g}$ at $4^{\circ} \mathrm{C}$ and protocol 
continued using the resulting pellet. All cytosolic and membrane samples were also only used a maximum of 3 times, to minimise the effects of freeze-thawing. Samples to be used more frequently than this were frozen in aliquots.

\subsubsection{Protein Quantification using the Lowry Method}

Protein was quantified using the Lowry method (Lowry et al. 1951). This is a 2step method. Firstly, copper ions complex with the protein, under alkaline conditions. Subsequently, Folin-Ciocalteau's phenol reagent is added and becomes reduced, changing from a yellow to a blue colour, which can be measured spectrophotometrically.

A bovine serum albumin (BSA) standard curve $(0-250 \mu \mathrm{g} / \mathrm{ml})$ was prepared by dilution of BSA stock solution of $250 \mu \mathrm{g} / \mathrm{ml}$ in $0.5 \mathrm{M} \mathrm{NaOH}$. $30 \mu \mathrm{l}$ of standards and samples (diluted as necessary in $0.5 \mathrm{M} \mathrm{NaOH}$ ) were pipetted, in triplicate, into separate wells of a clear flat-bottomed 96-well plate. Blanks were also prepared, in triplicate, containing $195 \mu \mathrm{l} 0.5 \mathrm{M} \mathrm{NaOH}$ (or protein extraction buffer at the appropriate dilution). $150 \mu$ l of freshly prepared copper solution ( $10 \mathrm{ml} 2 \%$ $\mathrm{Na}_{2} \mathrm{CO}_{3}, 0.1 \mathrm{ml} 1 \% \mathrm{CuSO}_{4} .5 \mathrm{H}_{2} \mathrm{O}, 0.1 \mathrm{ml} 2 \% \mathrm{KNaC}_{4} \mathrm{H}_{4} \mathrm{O}_{6} \cdot 4 \mathrm{H}_{2} \mathrm{O}$ ) was added to each well of standard or sample and the plate was left to stand, at room temperature, for 10 minutes. Then $15 \mu$ of Folin-Ciocalteau's phenol reagent ( $2 x$ stock diluted 1:1 in distilled water) was added to each well of standard or sample and mixed well. Plate was left for at least 30 minutes, at room temperature, and absorbance read at $490 \mathrm{~nm}$ on a Bio-Tek ELx800 microplate reader. Values were corrected for background absorbance, by subtracting average absorbance value for the blanks, and protein concentrations were determined by comparison to the standard curve. 


\subsubsection{Concentrating Protein Samples}

Samples were concentrated, as needed, prior to analysis. $0.1 \mathrm{ml} 0.15 \%$ deoxycholate was added to each $1 \mathrm{ml}$ protein sample. This was mixed using a vortex and left to stand at room temperature for 10 minutes. Then, $0.1 \mathrm{ml} 72 \%$ trichloroacetic acid was added and again mixed by vortexing, followed by centrifugation for 30 minutes at $11,000 \mathrm{~g}$ at $4^{\circ} \mathrm{C}$. Supernatant was removed and pellet was resuspended in required volume of $\mathrm{NaOH}$, to allow protein concentration to be remeasured using the Lowry method (section 2.2.3.3). In order to neutralise the alkaline before further analysis, an equal volume of $1 \mathrm{M}$ $\mathrm{Tris} / \mathrm{HCl}$ was then added to each sample.

\subsubsection{Separation of Proteins by SDS-PAGE}

Proteins were separated according to their molecular weight by sodium dodecyl sulphate polyacrylamide gel electrophoresis (SDS-PAGE). For proteins 40-100 kilodaltons $(\mathrm{kDa})$ in size a resolving gel of $12.5 \%$ (12.5\% acrylamide, $0.3 \%$ bisacrylamide, $0.1 \%$ SDS, $0.4 \mathrm{M}$ Tris- $\mathrm{HCl} \mathrm{pH} 8.8,0.16 \%$ ammonium persulphate (APS) and $0.16 \%$ tetramethylethylenediamine (TEMED)) and a stacking gel of $6 \%$ (6\% acrylamide, $0.16 \%$ bisacrylamide, $0.1 \%$ SDS, $0.2 \mathrm{M}$ Tris- $\mathrm{HCl} \mathrm{pH} 6.8$, $0.24 \%$ APS, $0.4 \%$ TEMED), made up in $1.5 \mathrm{~mm}$ glass plates, were used. For proteins less than $40 \mathrm{kDa}$ in size, a $20 \%$ resolving gel was used $(20 \%$ acrylamide, $0.53 \%$ bisacrylamide, $0.1 \%$ SDS, $0.33 \mathrm{M}$ Tris- $\mathrm{HCl} \mathrm{pH} 8.8,0.16 \%$ ammonium persulphate (APS) and $0.16 \%$ TEMED). Protein samples (amount used specified in individual figure legends) were mixed with an equal volume of loading buffer $(60 \mathrm{mM}$ Tris- $\mathrm{HCl} \mathrm{pH} 6.8,12 \%$ glycerol, $2 \% \mathrm{SDS}, 0.003 \%$ pyronin $\mathrm{Y}, 0.7 \mathrm{M} \beta$-mercaptoethanol (BME)) and heat denatured at $60^{\circ} \mathrm{C}$ for 10 minutes before being placed on ice. Denatured protein samples were then loaded onto the gel, alongside $10 \mu \mathrm{l}$ Novex Sharp Protein Standard. Gels were run at $120 \mathrm{~mA}$ in $1 \mathrm{x}$ running buffer $(25 \mathrm{mM}$ Tris- $\mathrm{HCl} \mathrm{pH} 8.3,192 \mathrm{mM}$ glycine, $0.1 \% \mathrm{SDS}$ ) for approximately 1 hour, or until the dye front neared the end of the glass plates. 


\subsubsection{Identification of Proteins by Western Blotting}

Separated proteins were transferred onto polyvinylidene difluoride (PVDF) membrane in $1 x$ transfer buffer $(16 \mathrm{mM}$ Tris base, $120 \mathrm{mM}$ glycine) at $300 \mathrm{~mA}$ for 90 minutes. The membrane was then left in blocking solution (10\% Marvel in 1x Tris-buffered saline - Tween 20 (TBST; $20 \mathrm{mM}$ Tris-HCl pH 7.5, $500 \mathrm{mM}$ $\mathrm{NaCl}, 0.1 \%$ Tween 20)) on a rotary mixer. Following blocking, the membrane was washed 3 times in $10 \mathrm{ml}$ TBST, each for 10 minutes, on a rotary mixer. The membrane was then incubated with primary antibody in TBST containing Marvel (see Table 2-5 for amount), followed by 3 further 10 minute washes in 1x TBST and incubation with secondary antibody ( $5 \%$ Marvel in $1 x$ TBST) at room temperature on a rotary mix. Three further 10 minute washes in 1x TBST were carried out, as before, prior to chemiluminescent detection using the ECL plus western blotting detection reagent. The membrane was incubated with the reagent for 5 minutes and then exposed to $x$-ray film. Conditions were optimised for each primary antibody; dilutions, amount of Marvel and times of incubation are shown in Table 2-5. All overnight incubation steps were carried out at $4^{\circ} \mathrm{C}$ and all other incubation steps carried out at room temperature. 


\begin{tabular}{|c|c|c|c|c|c|}
\hline Antibody & $\begin{array}{c}\text { Size } \\
\text { (kDa) }\end{array}$ & Blocking & $1^{\circ}$ & $2^{\circ}$ & $\begin{array}{l}\text { Exposure } \\
\text { (minutes) }\end{array}$ \\
\hline $\begin{array}{l}\text { MYOG } \\
\text { F5D }\end{array}$ & 25 & overnight & $\begin{array}{c}1: 25 \text {; overnight } \\
0.5 \% \text { Marvel }\end{array}$ & $\begin{array}{c}1: 20,000 ; 2 \mathrm{hr} \\
\alpha \text {-mouse } \\
(\mathrm{sc}-2005)\end{array}$ & 1 \\
\hline $\begin{array}{l}\text { MYH3 } \\
\text { F1-652 }\end{array}$ & 224 & overnight & $\begin{array}{c}\text { 1:25; overnight } \\
0.5 \% \text { Marvel }\end{array}$ & $\begin{array}{c}1: 20,000 ; 2 \mathrm{hr} \\
\alpha \text {-mouse }\end{array}$ & 15 \\
\hline $\begin{array}{c}\text { HMGCR } \\
(\mathrm{H}-300) \\
\text { sc-33827 }\end{array}$ & 90 & $1 \mathrm{hr}$ & $\begin{array}{c}\text { 1:400; overnight } \\
0.5 \% \text { Marvel }\end{array}$ & $\begin{array}{c}1: 10,000 ; 2 \mathrm{hr} \\
\alpha \text {-rabbit (sc-2004) }\end{array}$ & 1 or 5 \\
\hline $\begin{array}{c}\text { FT } \beta(H-300) \\
\text { sc-13965 }\end{array}$ & 46 & $1 \mathrm{hr}$ & $\begin{array}{c}\text { 1:1000; overnight } \\
0.5 \% \text { Marvel }\end{array}$ & $\begin{array}{c}1: 20,000 ; 1 \mathrm{hr} \\
\alpha-\text { rabbit }\end{array}$ & 1 \\
\hline $\begin{array}{c}\text { GGTase-I } \beta \\
(X X 12) \\
\text { sc-100820 }\end{array}$ & 42 & $1 \mathrm{hr}$ & $\begin{array}{c}1: 200 ; \text { overnight } \\
0.5 \% \text { Marvel }\end{array}$ & $\begin{array}{l}1: 2000 ; 2 h r \\
\alpha \text {-mouse }\end{array}$ & 25 \\
\hline $\begin{array}{c}\text { GGTase-II } \beta \\
(17-Q) \\
\text { sc-100819 }\end{array}$ & 43 & $1 \mathrm{hr}$ & $\begin{array}{c}\text { 1:200; overnight } \\
0.5 \% \text { Marvel }\end{array}$ & $\begin{array}{l}1: 2000 ; 2 \mathrm{hr} \\
\alpha \text {-mouse }\end{array}$ & 15 \\
\hline $\begin{array}{c}\text { H-Ras (M90) } \\
\text { sc-53959 }\end{array}$ & 21 & $1 \mathrm{hr}$ & $\begin{array}{c}\text { 1:500; overnight } \\
0.5 \% \text { Marvel }\end{array}$ & $\begin{array}{l}1: 5000 ; 2 \mathrm{hr} \\
\alpha \text {-mouse }\end{array}$ & 10 \\
\hline $\begin{array}{c}\text { M-Ras (N19) } \\
\text { sc-8168 }\end{array}$ & 29 & $1 \mathrm{hr}$ & $\begin{array}{c}\text { 1:750; overnight } \\
0.5 \% \text { Marvel }\end{array}$ & $\begin{array}{c}1: 5000 ; 2 \mathrm{hr} \\
\alpha \text {-goat (sc-2020) }\end{array}$ & 30 or 50 \\
\hline $\begin{array}{c}\text { Rap1A (C17) } \\
\text { sc-1482 }\end{array}$ & 21 & $1 \mathrm{hr}$ & $\begin{array}{c}1: 200 ; \text { overnight } \\
0.5 \% \text { Marvel }\end{array}$ & $\begin{array}{c}1: 10,000 ; 2 \mathrm{hr} \\
\alpha \text {-goat } \\
\end{array}$ & 5 or 20 \\
\hline $\begin{array}{l}\text { Rap1 (121) } \\
\quad \text { sc-65 }\end{array}$ & 21 & $1 \mathrm{hr}$ & $\begin{array}{c}\text { 1:200; overnight } \\
0.5 \% \text { Marvel }\end{array}$ & $\begin{array}{c}\text { 1:5,000; } 2 \mathrm{hr} \\
\alpha-\text { rabbit }\end{array}$ & 10 or 20 \\
\hline $\begin{array}{c}\text { Rab1A (C-19) } \\
\text { sc-311 }\end{array}$ & $\sim 25$ & $1 \mathrm{hr}$ & $\begin{array}{c}1: 500 ; \text { overnight } \\
0.5 \% \text { Marvel }\end{array}$ & $\begin{array}{c}1: 5000 ; 2 \mathrm{hr} \\
\alpha \text {-rabbit }\end{array}$ & 10 \\
\hline $\begin{array}{l}\beta \text {-actin } \\
\text { A5441 }\end{array}$ & 40 & overnight & $\begin{array}{l}\text { 1:2000; } 1 \mathrm{hr} \\
5 \% \text { Marvel, }\end{array}$ & $\begin{array}{c}1: 20,000 ; 2 \mathrm{hr} \\
\alpha \text {-mouse }\end{array}$ & $1-5$ \\
\hline $\begin{array}{c}\text { LDH } \\
a b 2101-1\end{array}$ & 35 & overnight & $\begin{array}{l}1: 1000 ; 2 \mathrm{hr} \\
1 \% \text { Marvel }\end{array}$ & $\begin{array}{c}1: 30,000 ; 1 \mathrm{hr} \\
\alpha-\text { goat }\end{array}$ & 30 \\
\hline
\end{tabular}

\section{Table 2-5 Antibody conditions for western blotting}

All overnight incubation steps were carried out at $4^{\circ} \mathrm{C}$, and all other incubation steps at room temperature. $1^{0}=$ primary antibody and $2^{\circ}=$ secondary antibody; $M Y O G=$ myogenin; $M Y H 3=$ myosin heavy chain 3; HMGCR = 3-hydroxy-3-methylglutaryl coenzyme A reductase; $L D H=$ lactate dehydrogenase; FT $=$ protein farnesyltransferase; GGTase-I = protein geranylgeranyltransferase type I; GGTase-II = protein geranylgeranyltransferase type II 


\subsubsection{Membrane Stripping and Reprobing}

To allow reprobing for $\beta$-actin as an internal loading control membrane was stripped of primary and secondary antibodies as follows: Membrane was incubated for 10 minutes on a rotary mix at room temperature with mild stripping buffer $(1.5 \%(w / v)$ glycine, $0.1 \%(w / v)$ SDS, $1 \%(v / v)$ Tween $20, \mathrm{pH} 2.2)$. This was repeated with fresh stripping buffer, followed by $2 \times 10$ minute washes with PBS and $2 \times 5$ minute washes with TBST. The membrane was blocked as before (see section 2.2.3.6) and probed with the new antibodies. Each membrane was stripped a maximum of 3 times, due to the loss of protein at each stage.

\subsubsection{Densitometry}

Densitometry was used to determine the relative sizes of protein bands obtained through western blotting (see section 2.2.3.6). Blots were scanned into the computer using a Xerox 2400 flatbed scanner, making sure to scan the images in greyscale and on the highest possible resolution, and saved as jpeg files. The graphics programme Paint was then used to select the area of the blot containing the protein bands of interest, and this selection was saved as a 24-colour bmp file. This bmp file was used in the programme GeneTools (Syngene, Cambridge, UK) to determine relative band sizes. Since this programme allows the user to select the exact area of the band, any background on the film should not interfere greatly with the results. Resulting data were normalised using the associated values obtained for the loading control $\beta$-actin.

\subsubsection{Measurement of Total Cellular Cholesterol Content}

In the cell, the majority of cholesterol is found in membranes (Brown and Goldstein 1997). Therefore, in order to quantify cellular cholesterol content, it is first necessary to extract it from these cell membranes. To achieve this, a variation of the Folch method was used (Folch et al. 1957) which is commonly used for the extraction of lipids (Gamble et al. 1978; Khan et al. 2003). 


\subsubsection{Extraction of Lipids from Cells}

RD cells were seeded at a density of $2 \times 10^{5}$ cells per well on 6-well plates and left to differentiate for 5 days in low-serum medium (RD-D; section 2.2.1.9). Huh7 and RD-U cells were seeded on 6-well plates 24 hours prior to experiment, at densities of $3 \times 10^{5}$ cells per well and $4 \times 10^{5}$ cells per well respectively. Cells were exposed to various statin concentrations or a vehicle control (section 2.2.1.11) for 48 hours. For control experiments, using only vehicle control, Huh7 cells were seeded in T25 flasks at either $8 \times 10^{5}$ cells per flask or at a range of densities between $4 \times 10^{5}$ and $1 \times 10^{6}$ cells per flask.

After the dosing period, medium was aspirated and cells washed twice in PBS. Cells were removed from the plate by trypsinisation (section 2.2.1.5), using $500 \mu \mathrm{l}$ trypsin-EDTA per well. $1 \mathrm{ml}$ complete medium was added and the resulting cell suspension transferred to an Eppendorf tube. Cells were pelleted in a micro centrifuge ( 5 minutes at $500 \mathrm{~g}$ ). The supernatant was removed and discarded, and the pellet washed in PBS, followed by further centrifugation to allow for any disturbance of the cell pellet during wash step. Supernatant was removed and discarded, and $200 \mu \mathrm{l}$ of a chloroform-methanol solvent mix (2:1 $\mathrm{v} / \mathrm{v}$ ) was added to each pellet, followed by brief vortexing. Samples were centrifuged for 10 minutes at $18,000 \mathrm{~g}$ in a micro centrifuge. Supernatant from this step (containing the lipid phase) was transferred to a new Eppendorf tube and dried under vacuum, to remove remaining solvent. Extracted lipids were then resuspended in $200 \mu \mathrm{l} 1 \mathrm{x}$ reaction buffer from the Amplex Red Cholesterol Assay Kit $(0.1 \mathrm{M}$ potassium phosphate, $\mathrm{pH} 7.4,50 \mathrm{mM} \mathrm{NaCl}, 5 \mathrm{mM}$ cholic acid, $0.1 \%$ Triton $\mathrm{X}-100)$. The pellet was resuspended in $0.5 \mathrm{M} \mathrm{NaOH}$, and protein quantification carried out using the Lowry method (section 2.2.3.3). All samples were stored at $-20^{\circ} \mathrm{C}$ until use.

\subsubsection{Cholesterol Quantification}

Total cholesterol was quantified using the Amplex Red Cholesterol Assay Kit. In this fluorometric method, cholesterol is oxidised by cholesterol oxidase to produce $\mathrm{H}_{2} \mathrm{O}_{2}$ and a ketone product. The probe Amplex Red (10-acetyl-3,7dihydroxyphenoxazine) reacts stoichiometrically with the $\mathrm{H}_{2} \mathrm{O}_{2}$, in the presence 
of horse radish peroxidase (HRP), to produce resorufin which can be detected using a fluorometer (Amundson and Zhou 1999; Sutter et al. 2005).

In brief, a cholesterol standard curve $(0-8 \mu \mathrm{g} / \mathrm{ml})$ and a positive control of $10 \mu \mathrm{M} \mathrm{H}_{2} \mathrm{O}_{2}$ were prepared by dilutions of the provided components into $1 \mathrm{x}$ reaction buffer (see section 2.2.4.1). 50 $5 \mathrm{l}$ of standards, positive control and samples (diluted as necessary in $1 \mathrm{x}$ reaction buffer) were pipetted, in triplicate, into separate wells of a 96-well black-bottomed microplate. An Amplex Red working solution was prepared ( $300 \mu \mathrm{M}$ Amplex Red reagent, $2 \mathrm{U} / \mathrm{ml} \mathrm{HRP}, 2 \mathrm{U} / \mathrm{ml}$ cholesterol oxidase, $0.2 \mathrm{U} / \mathrm{ml}$ cholesterol esterase) and $50 \mu \mathrm{l}$ added to each microplate well. Each plate was incubated for at least 30 minutes at $37^{\circ} \mathrm{C}$, protected from light, and fluorescence was measured in a SpectraMax Gemini XS microplate spectrofluorometer (Molecular Devices, California, USA) (excitation $544 \mathrm{~nm}$, emission $590 \mathrm{~nm}$ ). Values were corrected for background fluorescence and cholesterol content determined by comparison to the standard curve. Cholesterol values were then normalised to the associated protein data (see section 2.2.4.1).

\subsubsection{Statistical Analysis of Data}

Where data from a single experiment was presented, mean and standard deviation (SD) were used where the number of repeat measurements was four or greater, whereas median and range were used where this number of repeats was less than four. Standard error of the mean (SEM) was used to show the precision of the mean, where data was derived from several repeat experiments (Festing and Altman 2002). Statistical significance was determined using the most relevant statistical test, as indicated, where $P<0.05$ was considered to be significant. In general, one-way analysis of variance (ANOVA) followed by Bonferroni post hoc test was used to compare data from multiple samples, grouped by a single factor, and unpaired t-test was used to compare data from two samples, for example treated and untreated cells. Two-way ANOVA was used to compare data grouped by two factors. All tests were carried out using GraphPad Prism version 5 (California, USA). 


\section{Characterisation of Human Liver and Muscle Cell Lines}

\subsection{Introduction}

Circadian rhythms are known to influence a wide variety of biological processes, including many aspects of metabolism. Diurnal variation in cholesterol metabolism has been shown to occur, in part due to circadian transcription of key regulatory genes in this pathway: 3-hydroxy-3-methylglutaryl coenzyme $A$ reductase (HMGCR), the enzyme inhibited by the statins; low-density lipoprotein receptor ( $L D L R$ ); and the cytochrome p450 (CYP) enzymes involved in bile acid synthesis (Zmrzljak and Rozman 2012). Circadian rhythms can also affect the toxicity of xenobiotics, due to rhythmic variation in pharmacokinetic and pharmacodynamic factors, and it has been shown that in certain cases adverse drug effects can be reduced by changing drug dosing schedules accordingly (Levi and Schibler 2007). To examine whether differences exist between the circadian aspects of statin efficacy and toxicity, and whether greater separation can consequently be achieved between the two, the temporal effects of statins were to be studied in vitro. Initially the most appropriate model system was identified and characterised prior to further study.

The use of immortalised cell lines is an attractive option for such in vitro modelling. Human cell lines have previously been used to model and study the effects of statins; the RD muscle cell line has been used to study the myotoxic effects of statins, while the HepG2 liver cell line was used to look at the cholesterol-lowering effects (Kobayashi et al. 2008). Therefore both of these cell lines are good candidates to model the effects of statin exposure in muscle and liver. In addition, since it is possible to observe rhythmic gene expression in cultured human cells following a serum pulse, it has been suggested that studying the peripheral clocks of human cultured cells provides a good in vitro model for studying the molecular oscillator in human tissues (Takiguchi et al. 2007).

The HepG2 liver cell line is derived from a human hepatocellular carcinoma and the rhythmic expression of core clock genes, clock-controlled genes and several 
CYP enzymes, has already been demonstrated in this cell line (Koyanagi et al. 2006; Matsunaga et al. 2008; Takiguchi et al. 2007). For this reason it should represent a good model for examining the rhythm-dependent effects of statins on the liver.

Circadian rhythmicity is less well characterised in skeletal muscle, compared to tissues such as liver, although some evidence has been observed (Zhang et al. 2009). Transcriptome profiling in mouse skeletal muscle has revealed 215 transcripts (out of 36,182 ) with a circadian pattern of expression; this included several important muscle-specific proteins such as the transcription factor myogenic differentiation 1 (MYOD1) and the ubiquitin ligase atrogin-1 (McCarthy et al. 2007). Robust circadian rhythms have recently also been demonstrated in the skeletal muscle of rats (Miyazaki et al. 2011), zebrafish (Amaral and Johnston 2012), horses (Martin et al. 2010) and in the Movas-1 mouse smooth muscle cell line (Chalmers et al. 2008).

However, to date circadian rhythmicity has not been studied in RD human skeletal muscle cells. In order to use this muscle cell line as a model for looking at the temporal effects of statin administration, the ability of this cell line to establish and maintain circadian rhythms must first be verified. Therefore, in this chapter the hypothesis that RD cells have molecular clocks that are capable of generating robust circadian gene expression was tested. 


\subsection{Characterisation of RD Cell Differentiation}

The RD muscle cell line is derived from a human embryonic rhabdomyosarcoma, a tumour of skeletal muscle origin, and these cells express a number of muscle-specific genes (Knudsen et al. 1998). The expression of several of these genes only occurs after the initiation of muscle cell differentiation (Knudsen et al. 1998). For this reason, RD muscle cell differentiation is initiated before their use in experiments (Nishimoto et al. 2003), as this will lead to a more accurate representation of skeletal muscle. A variety of different differentiation conditions have been used in the literature (Bouche et al. 2000; Carey et al. 2006; Knudsen et al. 1998; Nishimoto et al. 2003; Vachon et al. 1996) and so before using these cells, the optimum differentiation conditions for this model must be determined.

\subsubsection{Expression of Muscle-Specific Markers}

In order to determine the optimum time required for differentiation, RD muscle cells were differentiated by incubation post-confluence in low (1\%) serum for between 2 and 10 days and the morphological and molecular changes were examined (section 2.2.1.9). Cell photographs, taken at 2-day intervals during this differentiation process (Figure 3-1), showed an increasing number of long myotube-like structures as differentiation progressed; the arrows in Figure 3-1 indicate examples of myotube formation occurring by days 2 and 4 . 


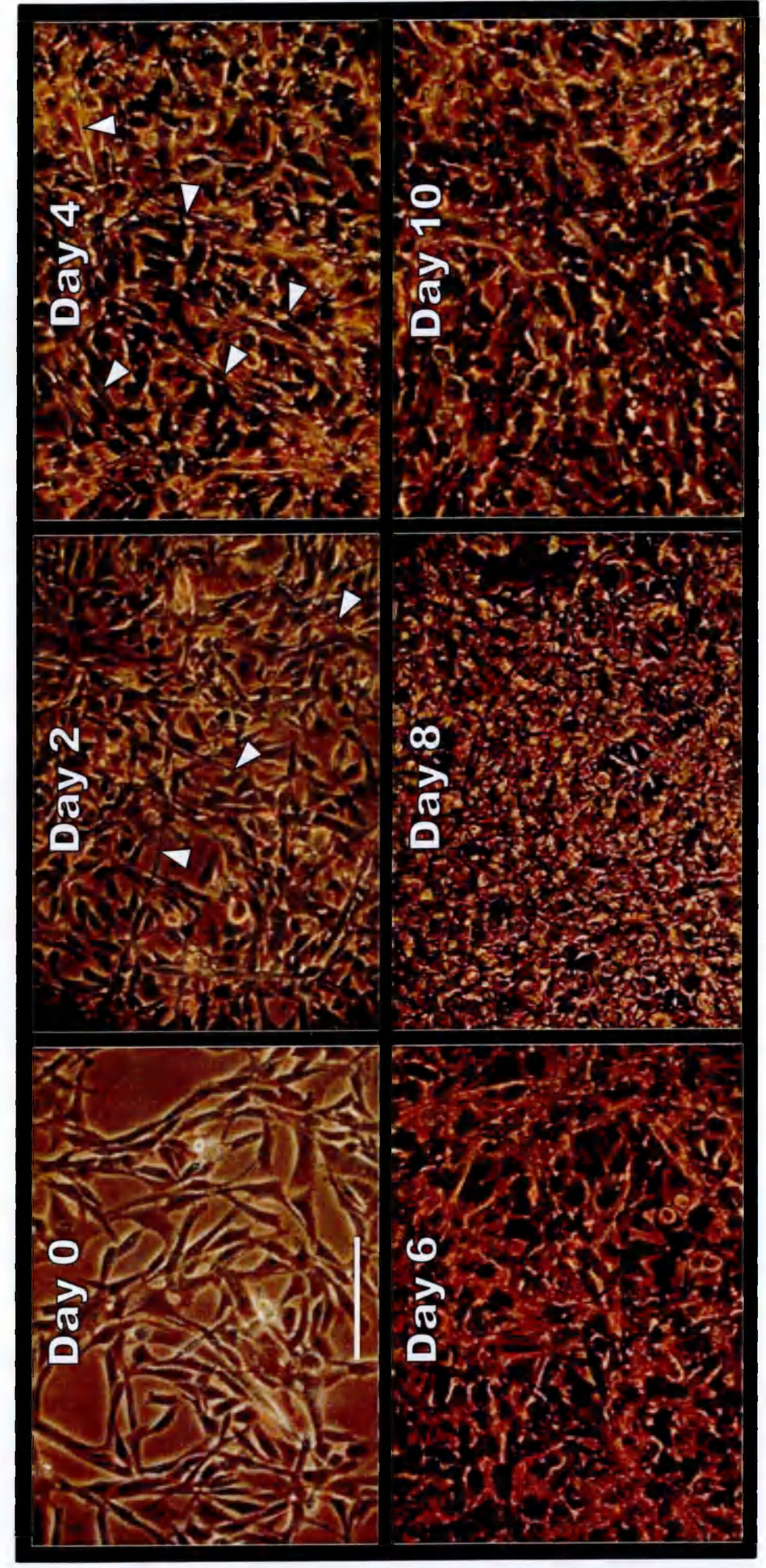


However, one property of this particular cell line is that on induction of differentiation these cells do not exit the cell cycle and so do not stop proliferating (Knudsen et al. 1998). Therefore, wells became overgrown in the later days of the time course, meaning it became difficult to monitor the progress of differentiation by microscopy. Due to this difficulty, the expression of several known muscle-specific markers was also measured using TaqMan (Figure 3-2).

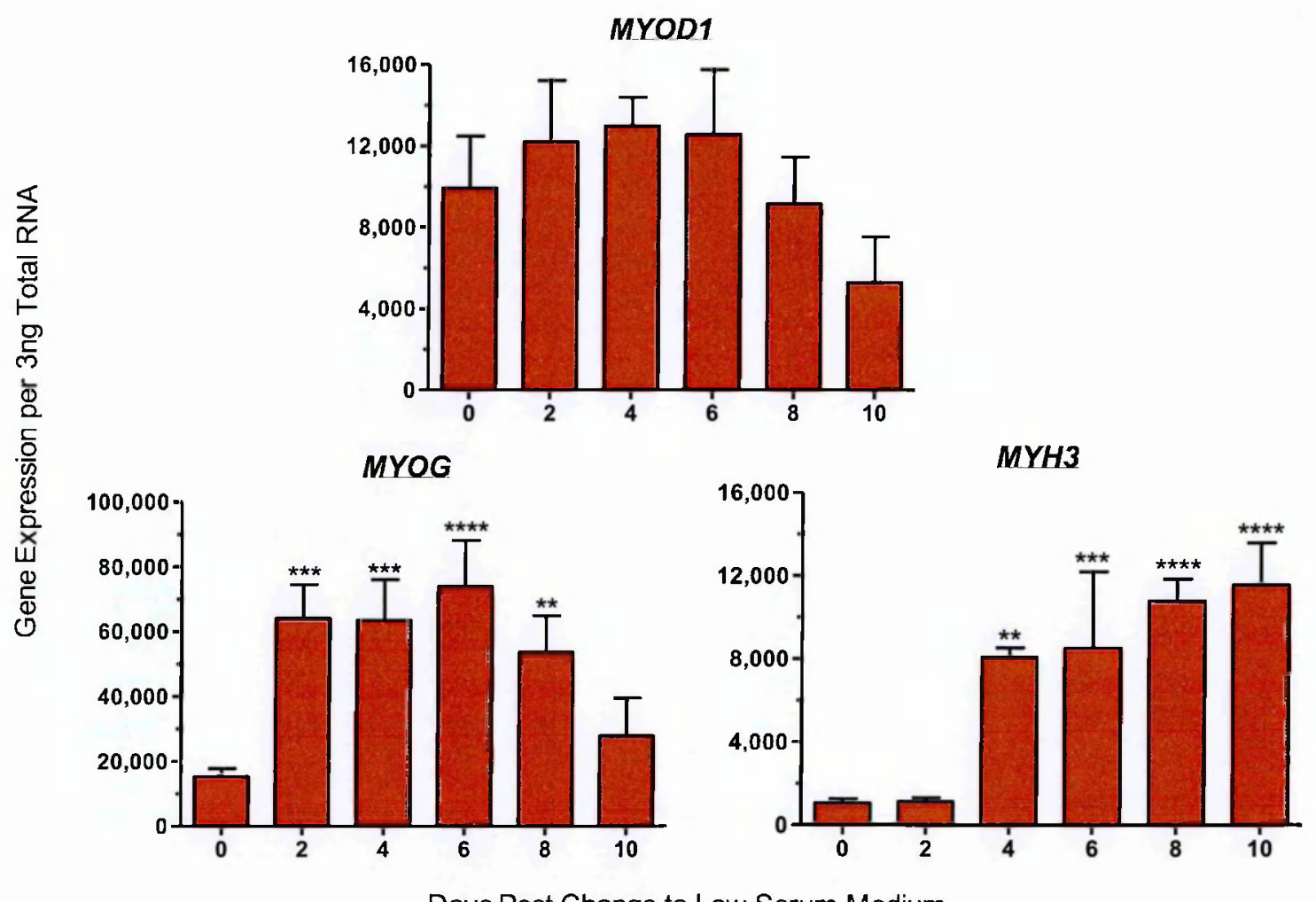

Figure 3-2 Molecular characterisation of RD muscle cell differentiation

$\mathrm{RD}$ cells were grown to confluence and then induced to differentiate by a change to low-serum (1\%) medium on day 0 . Total RNA was extracted from cells and reverse transcribed using random hexamers and the enzyme Superscript II Reverse Transcriptase (Invitrogen). Expression of three muscle markers, myogenic differentiation 1 (MYOD1), myogenin (MYOG) and myosin heavy chain $3(M Y H 3)$, was measured by quantitative RT-PCR (TaqMan), using comparison with genomic DNA at known concentrations (copy number) to enable quantification. Values plotted represent mean \pm standard deviation (SD), from a single experiment where $N=4$. Statistical significance determined by one-way ANOVA followed by Bonferroni post hoc test: ${ }^{* *}=\mathrm{P}<0.01,{ }^{* \star *}=\mathrm{P}<0.001,{ }^{* \star * *}=\mathrm{P}<0.0001$ compared to day 0 . 
TaqMan analysis showed that RD cells express the muscle-specific transcription factors MYOD1 and myogenin (MYOG). A statistically significant 4-fold increase in the expression of MYOG was observed between day 0 and day 2 of differentiation ( $P<0.001$, one-way analysis of variance (ANOVA) with Bonferroni post hoc test). This increase occurred in the same time scale as the observed morphological changes. In addition, the level of skeletal musclespecific myosin heavy chain 3 (MYH3) was very low prior to differentiation (day $0)$; however, there was a statistically significant 7-fold increase in $M Y H 3$ expression between days 2 and 4 of differentiation $(P<0.01$, Bonferroni post hoc test). MYOD1 was found to already be expressed at day 0 , with no significant change in expression observed early in the differentiation process, although a significant decrease in both MYOG and MYOD1 expression was observed by day 10 ( $p<0.05$ when comparing day 10 to days 2,4 and 6 for expression of both genes; Bonferroni post hoc test.)

These results indicate that cell differentiation has already begun after incubation for 2 days in low serum medium, when MYOG expression increases and myotubes are observed in culture, and that differentiation is complete by day 4 , when $M Y H 3$ is being expressed.

Western blot analysis showed that the transcript level changes were mirrored at the level of protein expression, following 5 days of differentiation (Figure 3-3).

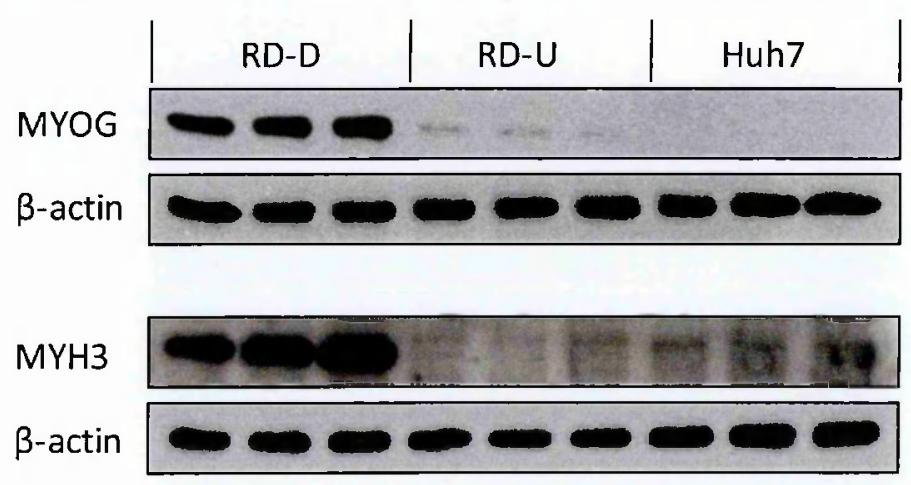

Figure 3-3 Protein expression of muscle-specific markers in Huh7 and RD cells

Total protein was extracted from Huh7 cells, undifferentiated RD cells (RD-U) and RD cells following 5 days of differentiation in $1 \%$ serum (RD-D). Protein concentration was determined using the Lowry method and $15 \mu \mathrm{g}$ was separated by SDS-PAGE. The levels of myogenin (MYOG) and myosin heavy chain $3(\mathrm{MYH} 3)$ were detected using specific antibodies, and $\beta-$ actin was used as a loading control; $\mathrm{N}=3$. Western blot analysis was carried out by Kate Plant and Jenny Spinks. 
Limited expression of myogenin was observed in undifferentiated RD cells (RD$U)$, but greater expression of myogenin protein was observed in cells that had been differentiated for 5 days in low-serum medium. No myogenin was detected in Huh7 liver cells. High MYH3 protein levels were observed in differentiated RD cells, but not in undifferentiated or Huh7 cells. This supports the data from microscopy and TaqMan analysis, and further supports the conclusion that it has been possible to differentiate RD cells here.

\subsubsection{Expression of Cholesterol Metabolism-Related Genes}

In order to determine whether differentiation would have an effect on cholesterol metabolism, the expression of HMGCR and LDLR was measured. TaqMan analysis confirmed the expression of both genes in undifferentiated RD cells, and there was no significant change in expression levels during the first 6 days post-differentiation (Figure 3-4).
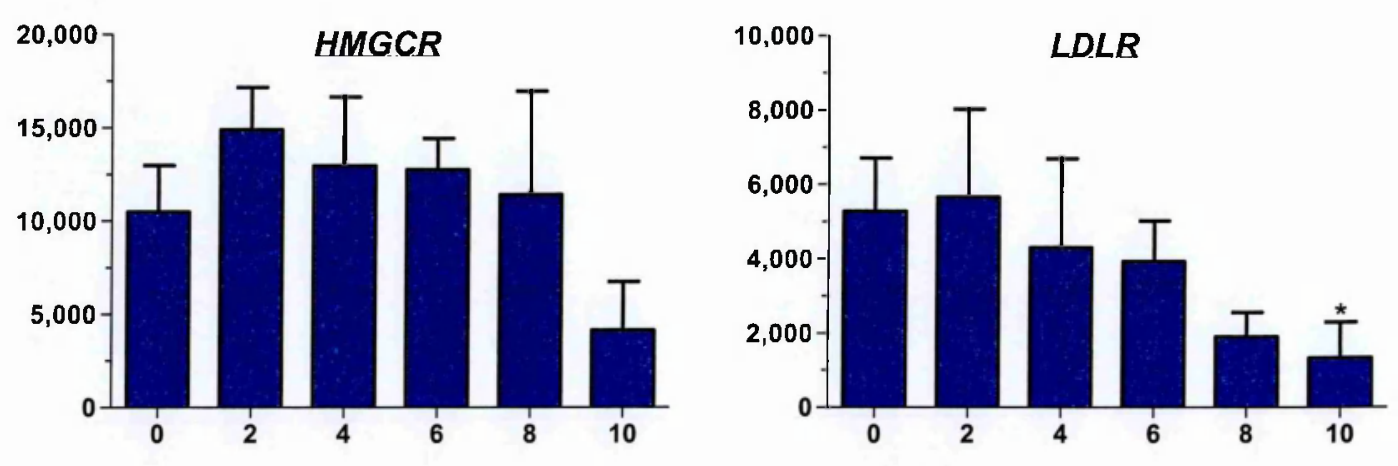

Days Post Change to Low Serum Medium

Figure 3-4 Cholesterol metabolism during RD muscle cell differentiation

$\mathrm{RD}$ cells were grown to confluence and then induced to differentiate by a change to low-serum differentiation medium on day 0 . Expression of 3-hydroxy-3-methylglutaryl coenzyme $A$ reductase (HMGCR) and low-density lipoprotein receptor ( $L D L r)$ was measured by quantitative RT-PCR (TaqMan). Values plotted represent mean \pm standard deviation (SD), from a single experiment where $N=4$. Statistical significance determined by one-way ANOVA followed by Bonferroni post hoc test: ${ }^{*}=P<0.05$ compared to day 0 .

However, both showed a significant decrease in expression following 10 days of differentiation, with approximately a 4-fold decrease in $L D L R$ expression $(\mathrm{P}<$ 0.05 when compared to days 0 and 2; Bonferroni post hoc test) and a 2.5 fold- 
decrease in HMGCR expression ( $P<0.05$ when compared with days 2, 4 and 6; Bonferroni post hoc test).

\subsubsection{Clock Gene Expression}

The expression of several key clock genes was also determined in order to both verify that RD cells express components of the molecular clock, since circadian rhythmicity has not previously been studied in this cell line, and to examine the effect of differentiation on these expression levels. TaqMan analysis confirmed the expression of brain and muscle arnt-like protein-1 (BMAL1), period 2 (PER2), cryptochrome 1 (CRY1) and D site of albumin promoter (albumin Dbox) binding protein (DBP) in RD cells (Figure 3-5).

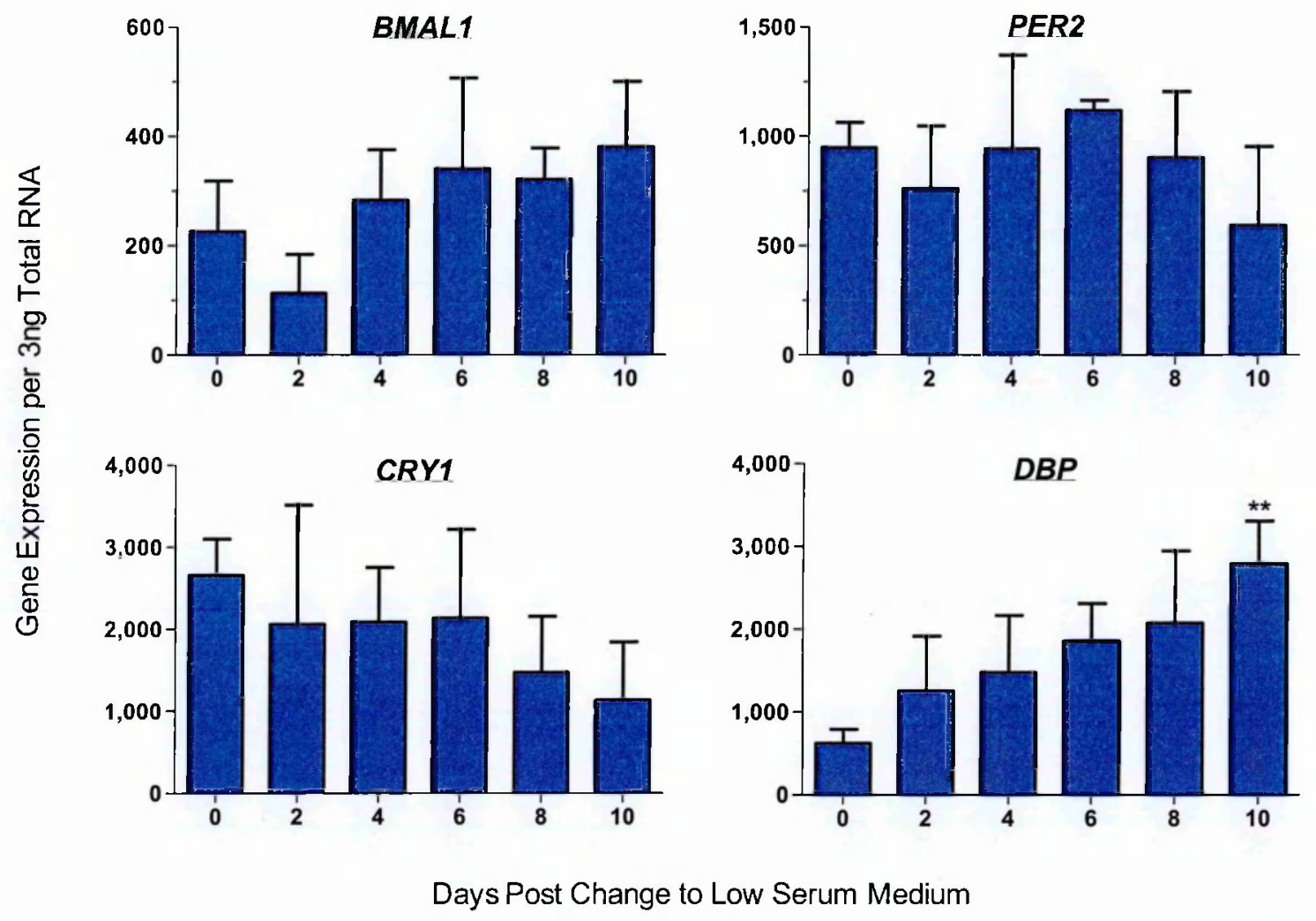

Figure 3-5 Expression of clock genes during RD muscle cell differentiation

$\mathrm{RD}$ cells were grown to confluence and then induced to differentiate by a change to low-serum differentiation medium on day 0 . Expression of the clock genes brain and muscle arnt-like protein-1 (BMAL1), period 2 (PER2), cryptochrome 1 (CRY1) and D site of albumin promoter (albumin D-box) binding protein (DBP) was measured by quantitative RT-PCR (TaqMan). Values plotted represent mean \pm standard deviation (SD), from a single experiment where $N=4$. Statistical significance determined by one-way ANOVA followed by Bonferroni post hoc test: ** $=P<0.01$ compared to day 0 . 
Levels of BMAL1, PER2 and CRY1 expression did not significantly change during the 10 days post-differentiation; however a 4.5-fold increase was observed between days 0 and 10 in the level of expression of $D B P(P<0.01$, Bonferroni post hoc test). This confirmation of clock gene expression suggests that there could be a functional molecular clock in these cells.

Taken together, these results show that RD cells can be induced to differentiate in low serum medium, and that this differentiation has occurred after 4 days. However, for many of the genes examined there is a significant decrease in expression following 8 or 10 days of differentiation. Therefore, for all subsequent experiments a differentiation time of 5 days was selected, since cells will have differentiated and this will allow an additional 48 hours for experiments prior to any significant decrease in gene expression. 


\subsection{Characterisation of Circadian Rhythms}

Having confirmed the expression of several key genes in the RD cell line and optimised cell differentiation, the next step was to study temporal gene expression in RD muscle cells. It is currently thought that there is an endogenous molecular clock in every individual cell; however, in the absence of external cues the circadian rhythms across a population of cells in culture are desynchronised (Nagoshi et al. 2004; Welsh et al. 2004). The application of a stimulus, such as a serum shock with $50 \%$ horse serum, has been shown to synchronise the rhythms of cells in culture, therefore enabling the analysis of circadian gene expression (Balsalobre et al. 1998). This method for synchronising cell rhythms is known as the serum pulse technique (section 2.2.1.10).

\subsubsection{Pilot Experiment}

Prior to studying circadian gene expression in RD cells, a pilot experiment was first carried out using 3 cell lines that are known to have previously shown circadian rhythmicity. As already described, rhythmic gene expression has previously been demonstrated in the HepG2 human liver cell line (Koyanagi et al. 2006; Matsunaga et al. 2008; Takiguchi et al. 2007). Rhythmic gene expression has been less well studied in the Huh7 human liver cell line, but nevertheless circadian rhythmicity has been demonstrated in the core clock gene PER1, the deadenylase nocturnin ( $\mathrm{Li}$ et al. 2008), and the cytokine thrombopoietin (Tracey et al. 2012). The 3T3-L1 murine preadipocyte cell line has also previously shown rhythmic expression of key clock genes (Otway et al. 2009).

Following a serum pulse, RNA was extracted at 4 time points to allow monitoring of gene expression over 36 hours, with time points selected for each cell line to match rhythmic expression profiles previously reported in the published literature (Koyanagi et al. 2006; Li et al. 2008; Otway et al. 2009). The time points were carefully chosen to enable observation of an expected peak of expression after 24-28 hours, as well as troughs after 12-16 and 36 hours, in 3T3-L1 and HepG2 cells but a peak after 16-20 as well as troughs after 8-12 and 24-32 hours in Huh7 cells. Expression of the representative core 
clock gene, Per2, and clock-controlled gene, Dbp, was analysed (Figure 3-6) since these genes often show the most robust rhythmic expression patterns in cultured cells (Otway et al. 2009).

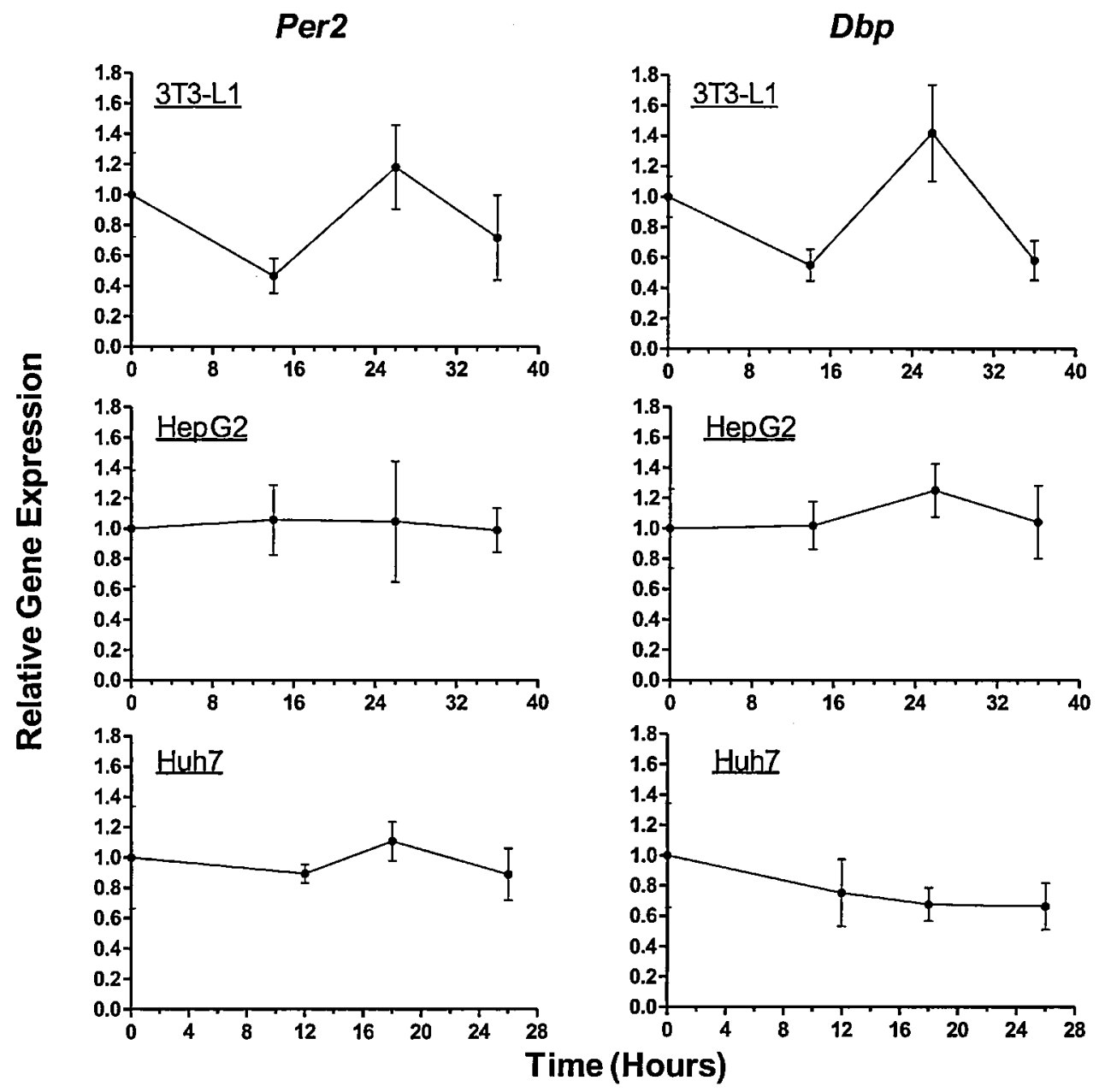

Figure 3-6 Temporai expression of PER2 and DBP in 3 different cell lines

3T3-L1, HepG2 and Huh7 cells were synchronised using a serum pulse (at $T=0$ ). RNA was extracted from 3T3-L1 and HepG2 cells at $T=0,14,26,36$ and from Huh7 cells at $T=0,12,18$ and 26. Temporal gene expression of period 2 (PER2) and D site of albumin promoter (albumin D-box) binding protein (DBP) was measured by quantitative RT-PCR (TaqMan). Unnormalised data are presented as mean \pm standard deviation $(S D)$, relative to expression at $T=0$, from a single experiment where $\mathrm{N}=4$.

TaqMan analysis revealed statistically significant rhythmic expression of both Per2 $(P=0.0072$, one-way analysis of variance (ANOVA)) and $D b p(P<$ 0.0001 , one-way ANOVA) in 3T3-L1 cells, with the expected peak at 26 hours and trough at 14 hours. 
There were no statistically significant changes observed in expression of PER2 and $D B P$ in either HepG2 cells $(P=0.9851$ and 0.3527 respectively, one-way ANOVA) or Huh7 cells ( $P=0.4590$ and 0.1793 respectively, one-way ANOVA).

Normalisation with the housekeeping genes $18 \mathrm{~S}$, glyceraldehyde 3-phosphate dehydrogenase $(G A P D H)$ or $\beta$-actin did not significantly alter expression patterns for either PER2 or DBP in any of the 3 cell lines used (two-way ANOVA; appendix 8.1).

The fact that rhythmic gene expression was detected in the 3T3-L1 cells indicated that the circadian rhythms of individual cells were being synchronised here by the serum pulse. It is possible that the lack of clear observation of rhythmic gene expression in the liver cell lines could be due to the small number of time points used in this experiment; therefore a more comprehensive analysis was carried out, using only 1 of the liver cell lines, the Huh7 cell line, alongside the RD cell line which has previously not been examined for circadian rhythmicity.

\subsubsection{Circadian Analysis in Huh7 and RD Cells}

A serum-pulse was used to synchronise both Huh7 (Figure 3-7) and RD-D (Figure 3-8) cells, and TaqMan analysis was used to examine gene expression over a longer time course of 56 hours, to enable the observation of 2 full 24hour cycles after cell synchronisation.

\subsubsection{Analysis of Temporal Gene Expression in Huh7 Cells}

In Huh7 liver cells (Figure 3-7), no statistically significant effect of time was seen on the expression of the core clock gene PER1 ( $P=0.6926$, one-way ANOVA). This is the only core clock gene that has previously been analysed for rhythmic gene expression in this cell line ( $\mathrm{Li}$ et al. 2008), with an observed peak in expression after 16-20 hours as well as troughs after 8-12 and 24-32 hours in both Huh7 and a variety of other cells types (Chalmers et al. 2008; Li et al. 2008; Wu et al. 2007). This rhythmic expression profile would also have been expected here. 


\section{Huh7}
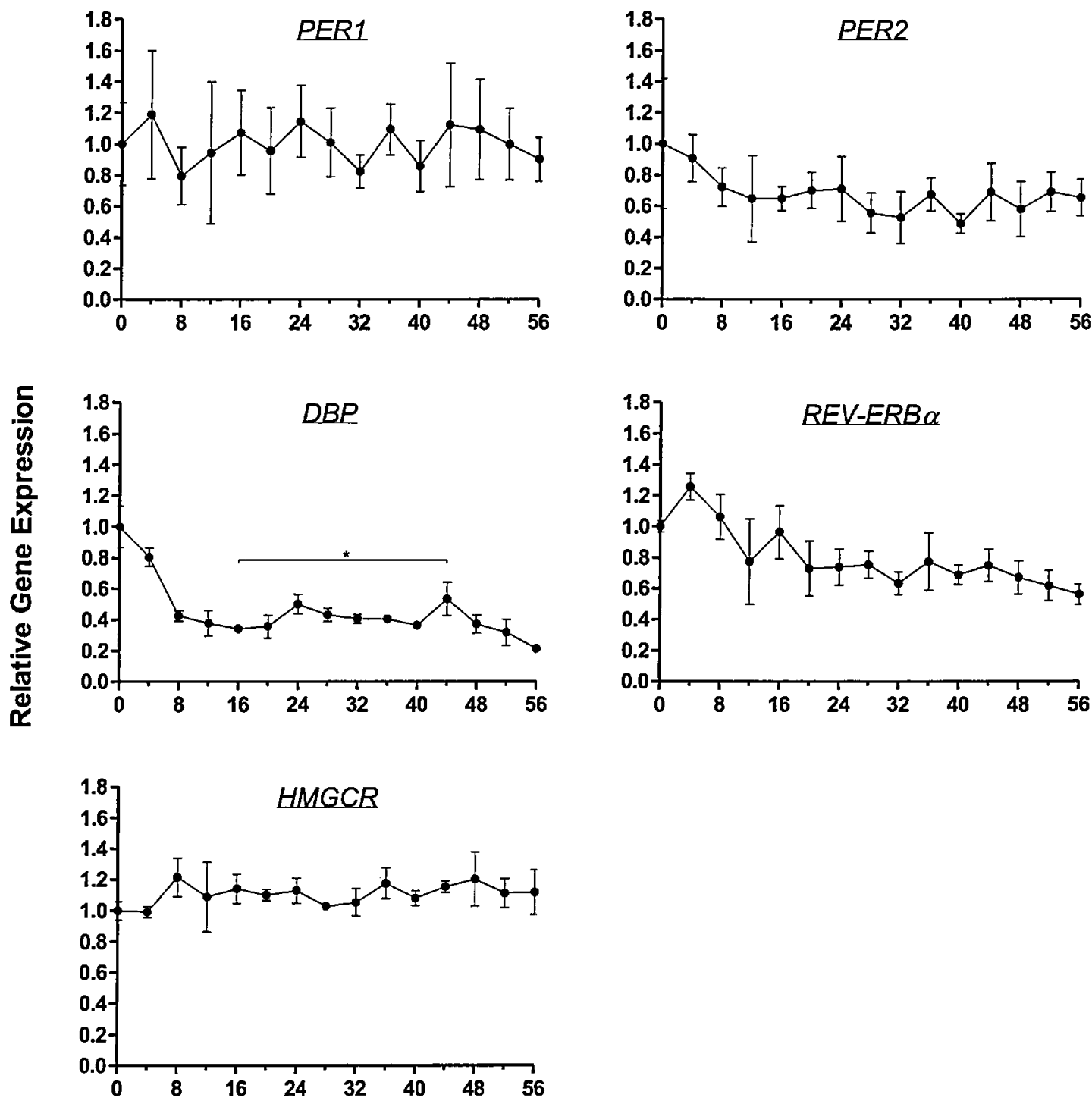

Time (hours)

Figure 3-7 Temporal gene expression in Huh7 liver cells

Huh7 cells were synchronised using a serum pulse (at $T=0$ ). RNA was extracted every 4 hours, for 56 hours, and temporal gene expression was measured by quantitative RT-PCR (TaqMan). Unnormalised data are presented as mean \pm standard deviation (SD), relative to expression at $T=0$, from a single experiment where $N=4$. Statistical significance determined by one-way ANOVA followed by Bonferroni post hoc test: ${ }^{*}=\mathrm{P}<0.05$.

Statistical significance for an effect of time on expression of the core clock gene PER2 was shown only between data points at time 0 and 40 hours after the serum pulse $(P<0.05$, Bonferroni post hoc test). However, since large changes in gene expression are often seen immediately after a serum pulse and prior to the observation of a circadian expression profile, this had no clear relevance for determination of rhythmic expression. Likewise, the only 
statistically significant changes in expression of the transcription factor reverse erythroblastosis virus a (REV-ERBa) were those based on data from time points immediately following the serum pulse ( $0-8$ hours) or on the 56 hour time point, when gene expression was starting to decrease at the end of the experiment.

HMGCR was included in this analysis since diurnal variation has been previously shown in both its transcription and enzyme activity in other models, and these variations potentially impact upon both statin efficacy and toxicity. However, there was no statistically significant effect of time on HMGCR gene expression in Huh7 cells in this analysis ( $P=0.1192$, one-way ANOVA).

Only $D B P$, a transcription factor that regulates the expression of many clockcontrolled genes, showed an indication of rhythmic variation in gene expression with peaks of expression at 24 and 44 hours. There was a statistically significant difference between expression at 16 and 44 hours $(P<0.05$, Bonferroni post hoc test), where these time points represent a trough and a peak respectively, and these observed peaks would be consistent with DBP rhythmic expression profiles reported elsewhere (Otway et al. 2009).

\subsubsection{Analysis of Temporal Gene Expression in RD-D Cells}

It has been shown that circadian gene expression profiles of key clock genes in skeletal muscle are frequently similar to those seen in liver (Miyazaki et al. 2011), and therefore the expectations for rhythmic variation of these genes in RD cells would be similar to those for Huh7 cells. However, in RD-D muscle cells (Figure 3-8) there was no statistically significant effect of time on the expression of the core clock genes CRY1, PER1, PER2, transcription factors $R E V-E R B \alpha$ and $D B P$, or HMGCR. This is after taking into account the large changes in expression, particularly in CRY1, PER1 and PER2, immediately following the serum pulse. 


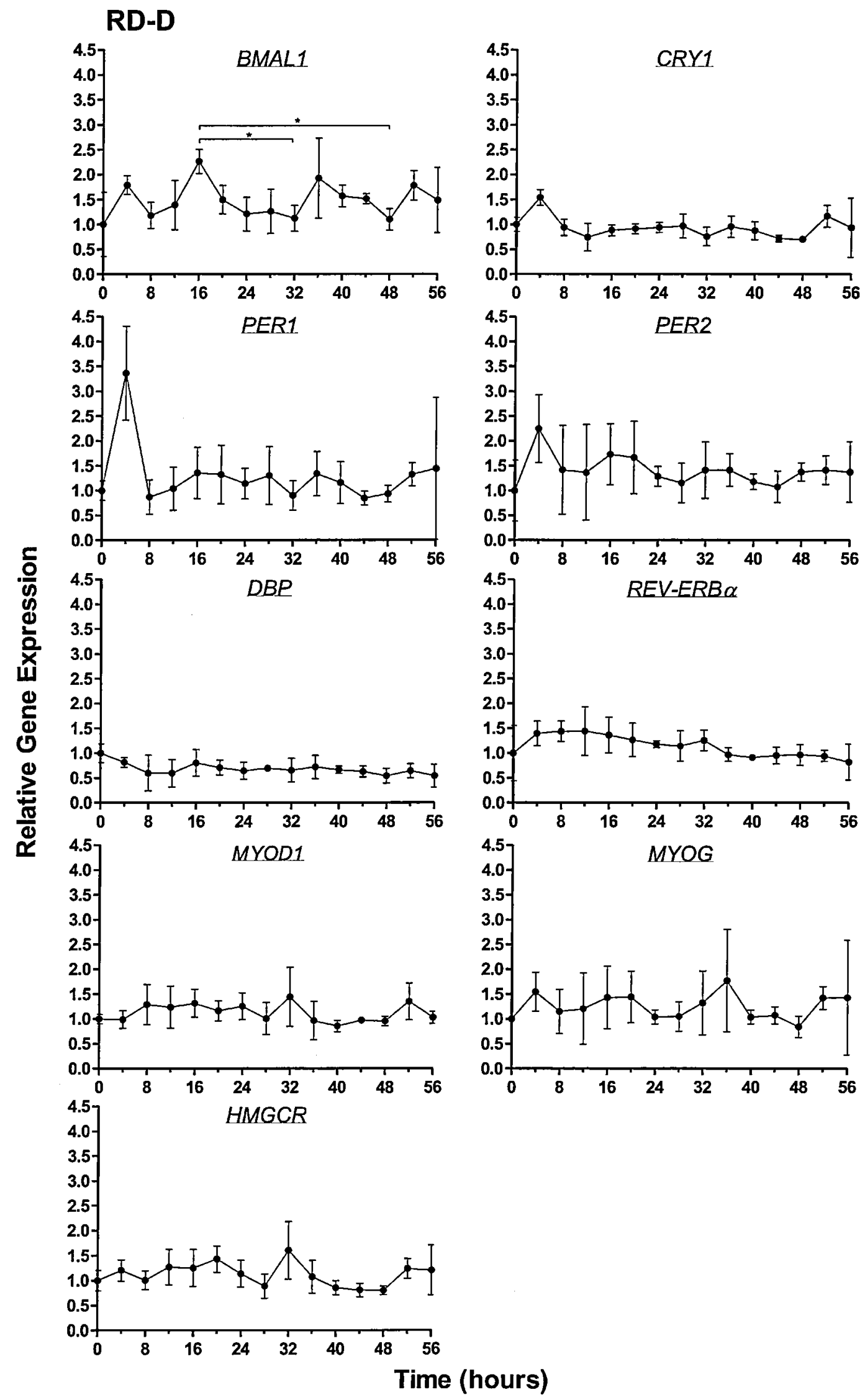

Figure 3-8 Temporal gene expression in RD-D muscle cells

RD-D cells were synchronised using a serum pulse (at $T=0$ ). RNA was extracted every 4 hours, over a time of 56 hours, and temporal gene expression was measured by quantitative RT-PCR (TaqMan). Unnormalised data are presented as mean \pm standard deviation (SD), relative to expression at $T=0$, from a single experiment where $N=4$. Statistical significance determined by one-way ANOVA followed by Bonferroni post hoc test: * $=\mathrm{P}<0.05$. 
There was also no statistically significant effect of time on the expression of muscle-specific transcription factors MYOD1 and MYOG. This is surprising, as an effect might have been expected since MyoD1 has shown circadian expression of RNA in rat skeletal muscle (Miyazaki et al. 2011) and circadian expression of both RNA and protein in mice (Andrews et al. 2010; Zhang et al. 2012).

The core clock gene BMAL1 has previously shown rhythmic gene expression in cultured cells, with expression peaks at 12-20 hours and 32-40 hours as well as troughs at 20-28 hours in both adipose-derived stem cells (Wu et al. 2007) and Movas-1 mouse smooth muscle cells (Chalmers et al. 2008). In this analysis with RD-D cells, statistical significance was found between BMAL1 gene expression at 16 hours, a potential peak, and gene expression at 32 and 48 hours, 2 potential troughs $(P<0.05$, one-way ANOVA).

\subsubsection{Normalisation of Gene Expression Data}

The gene expression data presented in Figure 3-7 and Figure 3-8 is not normalised; however, in quantitative PCR ( $\mathrm{PPCR}$ ) studies it is common for data to be normalised to the expression of internal control genes (often referred to as "housekeeping genes"). The use of internal control genes for the normalisation of such data is primarily to minimise any error that may occur from discrepancies in the initial cDNA input, which may arise from differing efficiencies of the cDNA synthesis reactions. These genes should not vary in expression in the tissue(s) or cell line(s) being investigated, or as a result of the experimental treatment (Vandesompele et al. 2002). 
For this analysis, $18 S$ ribosomal RNA (rRNA) and $\beta$-actin were chosen as housekeeping genes. 18S rRNA has commonly been used for normalisation, and hence was also tried here. $\beta$-actin has been shown to exhibit very little circadian variation in several tissues when compared to other housekeeping genes (Kamphuis et al. 2005) and it has been used previously as an internal control for experiments in RD cells (Phillips et al. 2007; Yang et al. 2009). TaqMan analysis was therefore carried out, but revealed large variations in the expression of $18 S$ and $\beta$-actin In both Huh7 and RD-D cells (Figure 3-9).

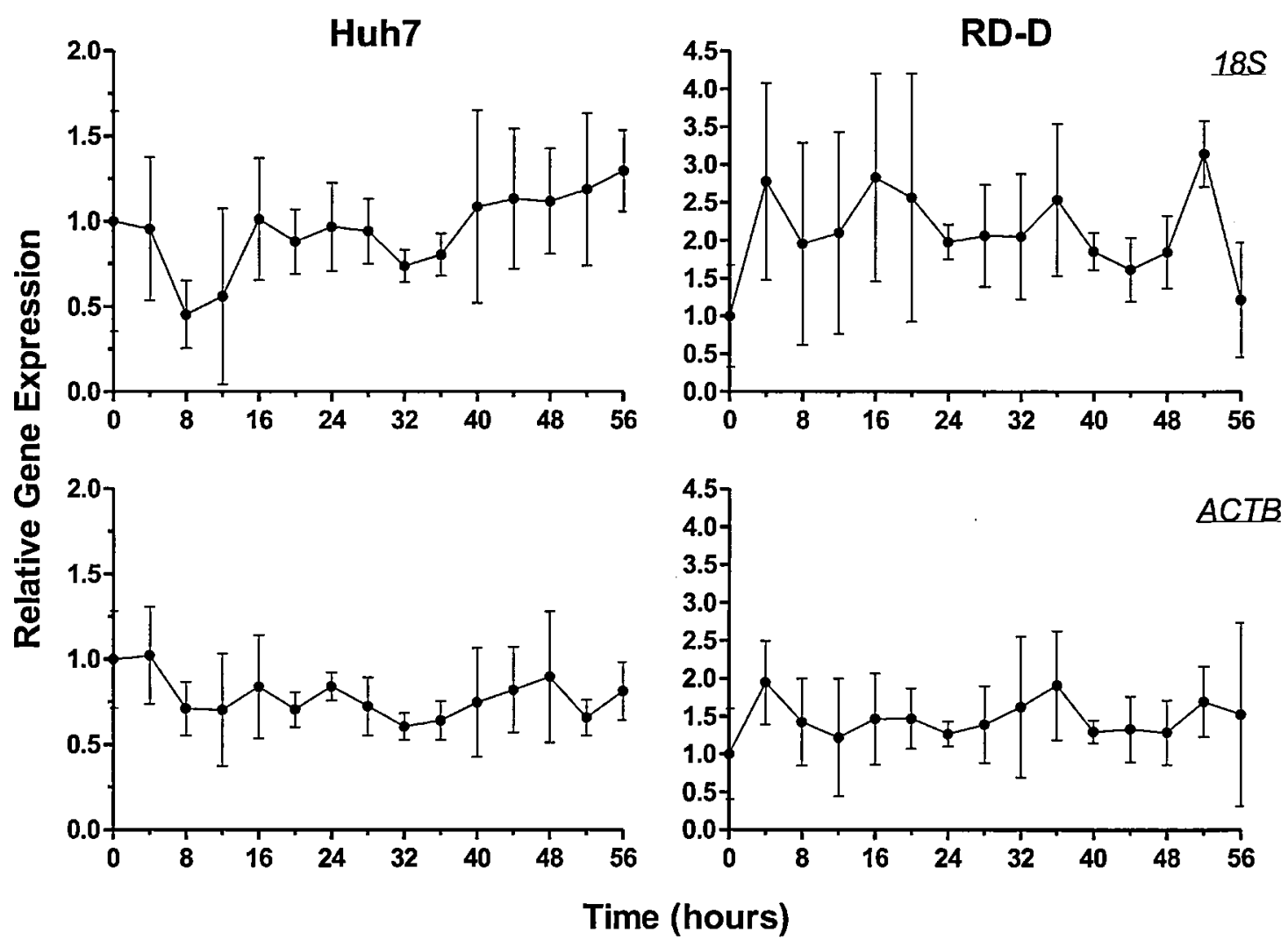

Figure 3-9 Temporal gene expression of two different housekeeping genes in Huh7 and RD-D cells

Huh7 and RD-D cells were synchronised using a serum pulse (at $T=0$ ). Temporal gene expression of housekeeping genes $18 \mathrm{~S}$ and $A C T B$ was measured by quantitative RT-PCR. Data are presented as mean \pm standard deviation (SD), relative to expression at $T=0$, from a single experiment where $\mathrm{N}=4$. 
Temporal gene expression data from both Huh7 (Figure 3-10) and RD-D (Figure 3-11) cells was normalised to either $18 \mathrm{~S}$ or $\beta$-actin. Initially data for the expression of PER2 and DBP was used since these were the two genes analysed previously in the pilot experiment.

Huh7
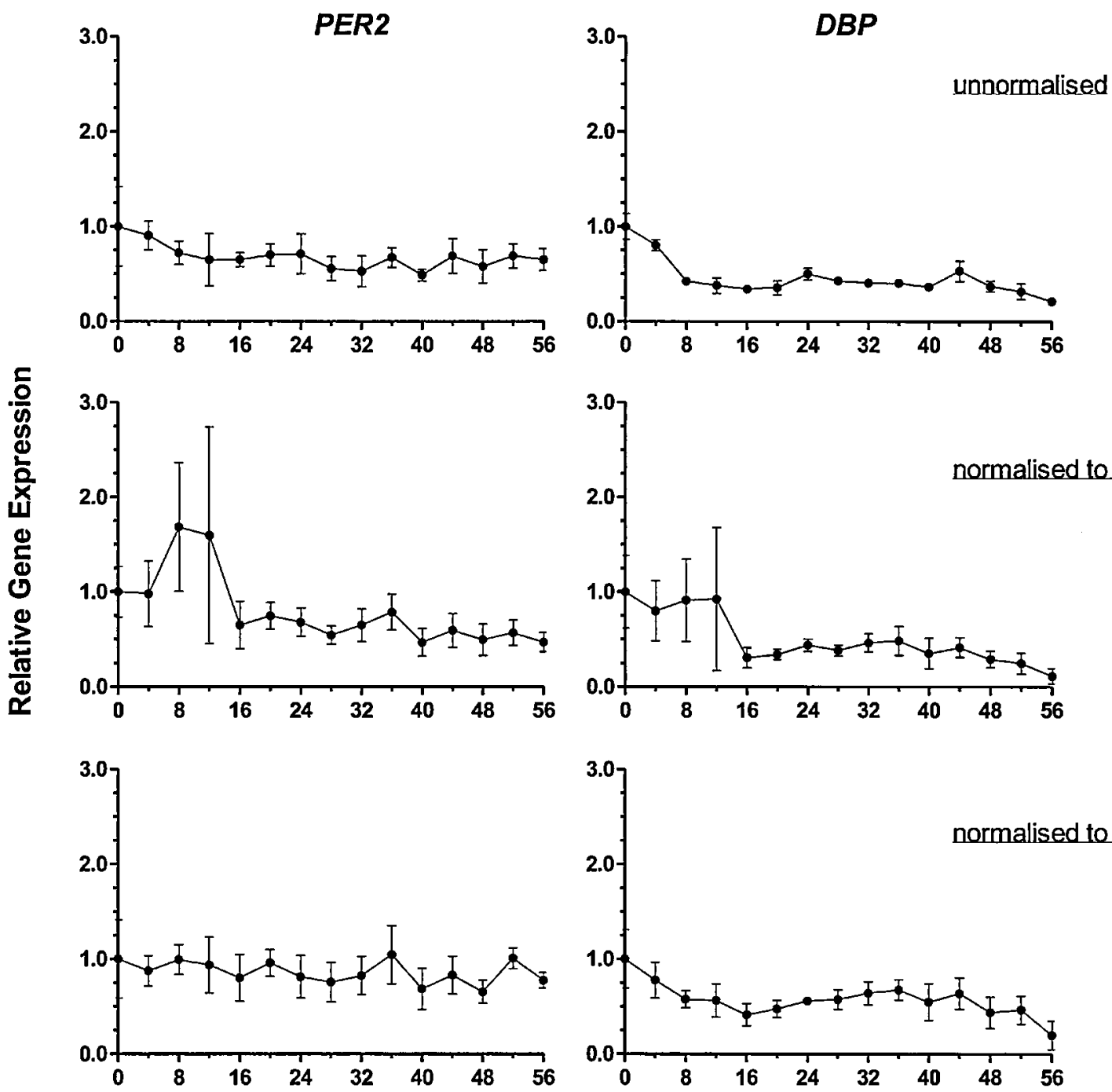

Time (hours)

Figure 3-10 Effect of normalisation of Huh7 TaqMan data using two different housekeeping genes

Huh7 cells were synchronised using a serum pulse (at $T=0$ ) and temporal gene expression of $P E R 2$ and $D B P$ was measured by quantitative RT-PCR. Data are shown unnormalised and normalised to $18 \mathrm{~S}$ or ACTB expression, and are presented as mean \pm standard deviation (SD), relative to expression at $\mathrm{T}=0$, from a single experiment where $\mathrm{N}=4$. 
Normalisation of data from Huh7 cells introduced new, large peaks in the expression profiles of $P E R 2$ and $D B P$, as well as greater overall variability within the dataset, as seen particularly in data points from $0-16$ hours when data was normalised to $18 \mathrm{~S}$. This was a direct reflection of the variation seen in the 18S TaqMan data, which could have arisen due to a genuinely large variation in the efficiencies of the individual reactions used to synthesize the cDNA used for TaqMan analysis or an actual variation in $18 \mathrm{~S}$ gene expression. Since the normalised data profile seemed to relate directly to the housekeeping gene expression, it would suggest that the latter may play a more important role and therefore data normalisation may have the potential to mask any genuine temporal effects.

Bearing this in mind, when time points $0-16$ were removed from the analysis there was no statistically significant difference in gene expression profiles for either PER2 ( $\mathrm{P}=0.8231$, two-way ANOVA) or DBP ( $\mathrm{P}=0.7657$, two-way ANOVA) between unnormalised data or data normalised to the housekeeping genes $18 S$ or $\beta$-actin. 


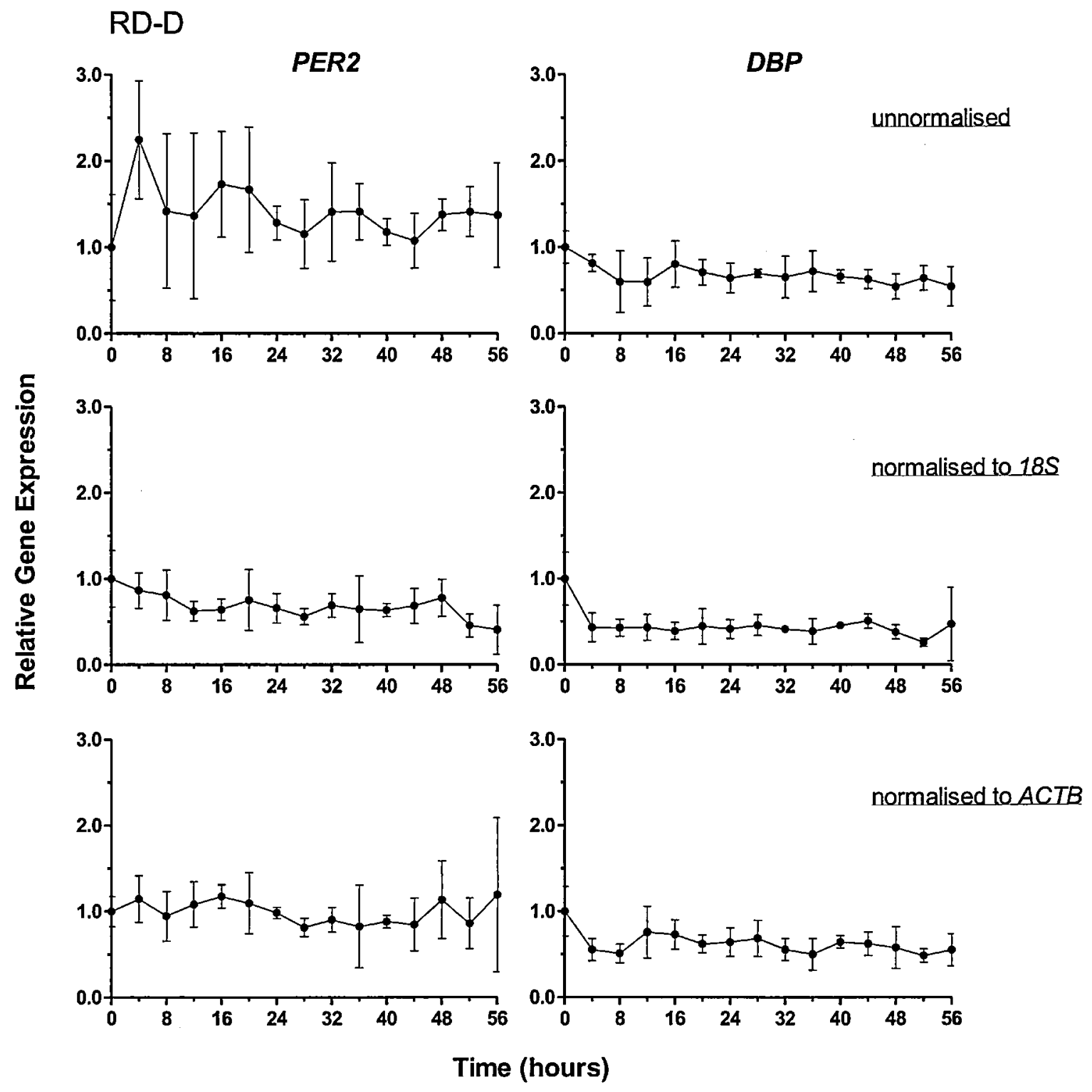

Figure 3-11 Effect of normalisation of RD-D TaqMan data using two different housekeeping genes

RD-D cells were synchronised using a serum pulse (at $T=0$ ) and temporal gene expression of $P E R 2$ and $D B P$ was measured by quantitative RT-PCR. Data are shown unnormalised and normalised to $18 S$ or $A C T B$ expression, and are presented as mean \pm standard deviation (SD), relative to expression at $\mathrm{T}=0$, from a single experiment where $\mathrm{N}=4$.

The normalisation of data from RD-D cells, to housekeeping gene $18 \mathrm{~S}$ or $\beta$ actin, also had no statistically significant effect on gene profiles of PER2 ( $P=$ 0.7699, two-way ANOVA) or DBP ( $P=0.9225$, two-way ANOVA). Similarly, normalisation of the other genes analysed had no significant effect on gene expression profiles and did not lead to any unmasking of any previously unseen 
circadian effects (appendix 8.2). Therefore, the decision was made to leave TaqMan data unnormalised.

In summary, no clear circadian rhythms of gene expression have been observed in either Huh7 (Figure 3-7) or RD-D (Figure 3-8) cells. Out of all of the genes examined, the only potentially significant temporal variations in gene expression were observed with the transcription factor DBP in Huh7 cells and the core clock gene BMAL1 in RD-D cells.

\subsubsection{Relative Abundance of Clock Genes}

Data obtained from the TaqMan analysis of temporal gene expression was used to compare the abundance of the different gene transcripts between Huh7 and RD-D cells (Figure 3-12). The data used was from the $T=0$ time point of the analysis, meaning that this represents transcript abundance prior to cells receiving a serum pulse.

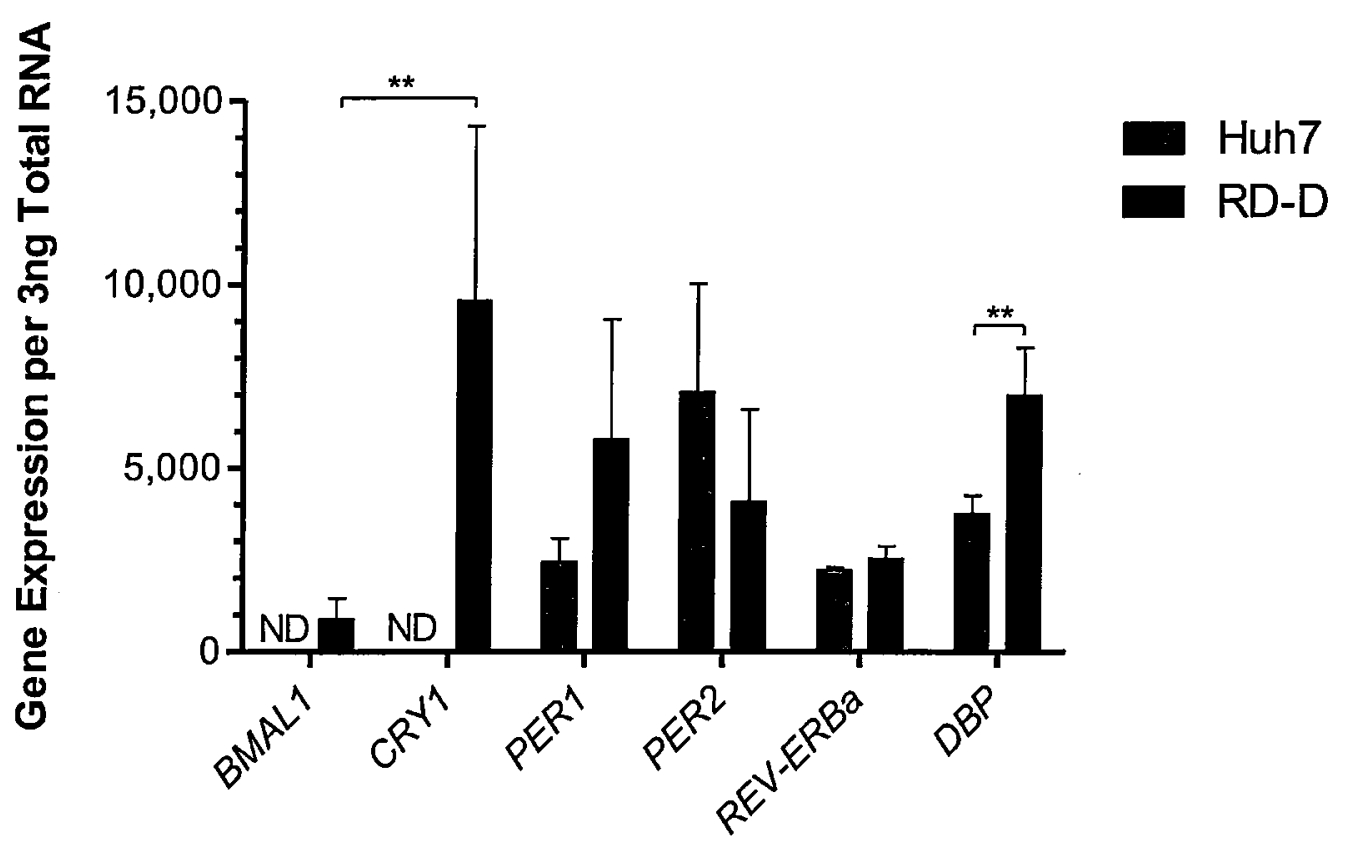

\section{Gene}

FIgure 3-12 Comparlson of gene expression In Huh7 liver and RD-D muscle cells

RT-PCR (TaqMan) data from $T=0$ time point of serum pulse experiments in Huh7 liver (Figure 3-7) and RD-D muscle (Figure 3-8) was used to compare the abundance of different genes between cell lines. Gene expression of BMAL1 and CRY1 was not determined (ND) in Huh7 liver cells. Data represent mean \pm standard deviation (SD), from a single experiment where $\mathrm{N}=4$ 
From this comparison, it seems that there is greater expression of the core clock gene PER1 in RD-D than in Huh7 cells $(P=0.0930$, unpaired two-tailed $t-$ test), whereas $P E R 2$ expression may be greater in Huh7 liver cells than in RD$\mathrm{D}(\mathrm{P}=0.1755$, unpaired t-test). REV-ERBa expression is similar in both Huh7 and RD-D cells ( $P=0.1981$, unpaired t-test), but there is significantly greater expression of the clock controlled transcription factor DBP in RD-D than Huh7 $(P=0.0058$, unpaired t-test). Expression levels of $C R Y 1$ and $B M A L 1$ were only analysed in RD-D cells. The level of CRY1 expression in RD-D cells was not significantly different from that of the other clock genes, apart from BMAL1 (one-way ANOVA). The core clock gene BMAL1 had the lowest expression, with a transcript level that was almost 3 times lower than the next lowest expressed transcript in RD-D cells, REV-ERBa. This is consistent with what was also observed during RD cell differentiation (Figure 3-5).

\subsection{Discussion}

In order to study the circadian aspects of statin-induced myopathy it is important to establish a robust in vitro model. For this reason the first stage of this project was to characterise RD muscle cell differentiation, by looking at the morphological and molecular changes that occurred during this process, followed by characterisation of circadian rhythms in this same cell line (objectives 1 and 2; section 1.5).

\subsubsection{RD Cell Differentiation}

Muscle cell differentiation is a highly ordered, multi-step process in which, on mitogen withdrawal, proliferating myoblasts differentiate into myocytes, which exit from the cell cycle and fuse to form multinucleated myotubes and myofibres that express a number of structural and enzymatic muscle-specific proteins (Andres and Walsh 1996). This process is regulated by four myogenic regulatory factors (MRFs): MYOD1, MYF5, MYOG, and MRF4, which are muscle-specific basic helix-loop-helix transcription factors (Moran et al. 2002) that are activated at differing stages of the differentiation process (Figure 3-13). MYOD1, a "primary MRF", is expressed early in the differentiation process. It is expressed both before and after differentiation in vitro and is thought to be important for myoblast determination in vivo (Megeney and Rudnicki 1995). 
MYOG, a so-called "secondary MRF", is expressed upon differentiation and then plays a role in terminal differentiation (Megeney and Rudnicki 1995). The results obtained here, both in terms of the observation of an increasing number of myotubes in the cell culture and the observed changes in gene expression, are consistent with the occurrence of RD cell differentiation.

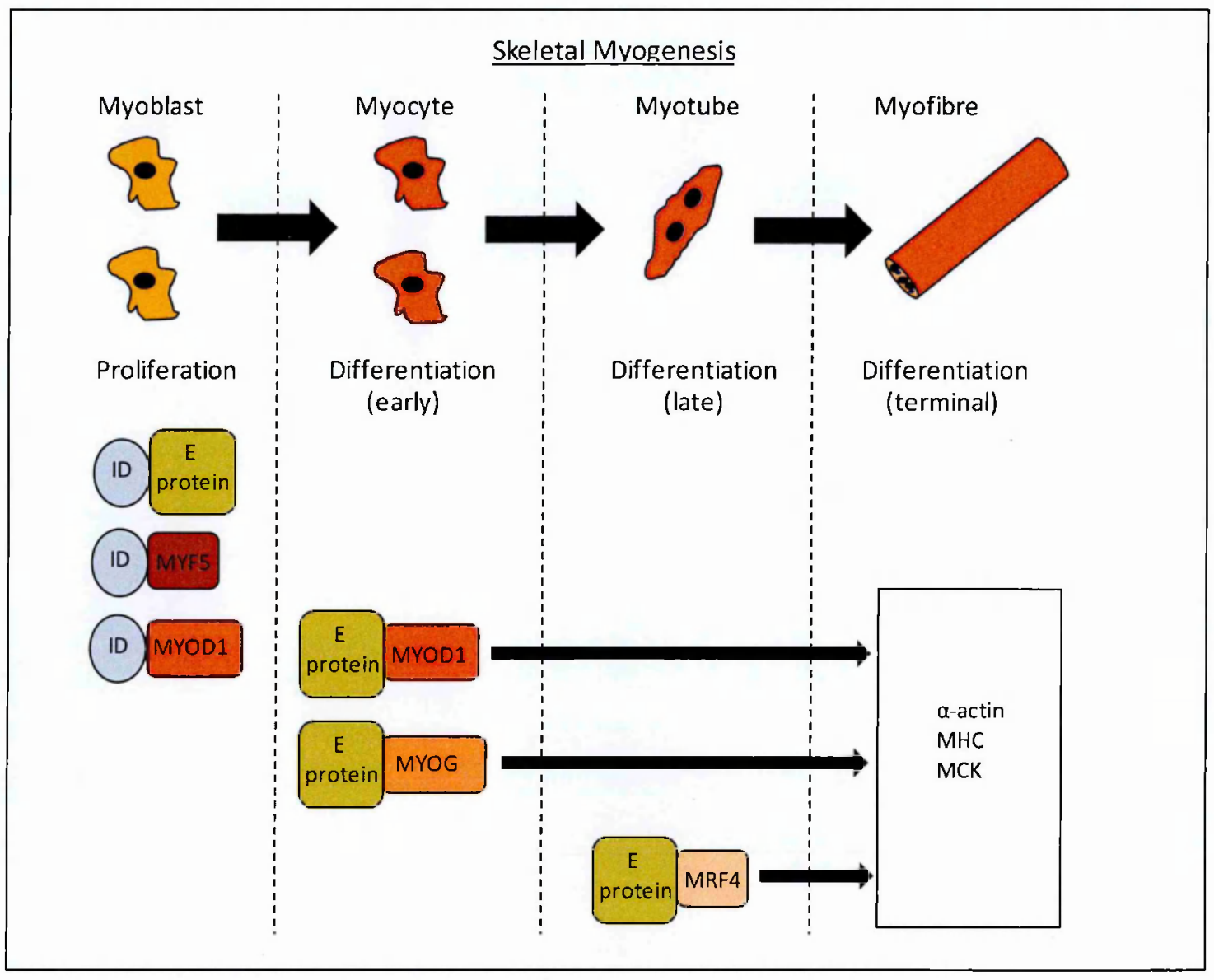

Figure 3-13 Regulation of skeletal myogenesis

Myogenesis is regulated by the myogenic regulatory factors (MRFs): MYF5, MYOD1, MYOG and MRF4, which are transcription factors that activate expression of muscle-specific genes, such as a-actin, myosin heavy chain (MHC) and muscle creatine kinase (MCK). On differentiation, the inhibitor of differentiation protein (ID) is downregulated, allowing MRFs to form functionally active heterodimers with the ubiquitously expressed $E$ proteins. Each MRF plays a role at a different stage of differentiation, as shown. MYOD1 and MYF5 are expressed early during muscle development and play a role in myoblast determination, whereas MYOG and MRF4 are expressed later in the differentiation process. Figure adapted from (Lluis et al. 2006). 
MYOD1 was seen to be expressed prior to, and throughout, differentiation. The former could suggest that even before RD cells are stimulated to fully differentiate they are committed to the skeletal-muscle cell lineage. A large increase in MYOG expression was noted 2 days after the change to low-serum medium, which would be consistent with the onset of early differentiation and the formation of myocytes. It is interesting to note that upon differentiation there is a difference between the extent of MYOG RNA induction and protein induction (Figure 3-2 and Figure 3-3). This indicates that there may be posttranscriptional regulation of myogenic regulatory factors, and shows the importance of examining changes in protein expression as well as in gene expression.

Myosin plays an important role in muscle contraction (Bandman 1992). It is a marker of terminal differentiation (Bennett and Tonks 1997) and myosin heavy chain $(\mathrm{MHC})$ has previously been used to monitor the differentiation status of RD cells (Ma et al. 2003). MYH3 is an embryonic skeletal muscle MHC isoform (Moran et al. 2002) and so its expression was examined here as the RD cell line originates from an embryonic rhabdomyosarcoma (Knudsen et al. 1998) and is known to express this MHC isoform (Rubin et al. 2011; Schiaffino et al. 1986; Yang et al. 2009). The large increase in MYH3 expression by day 4 of RD cell differentiation is similar to what has previously been observed during differentiation of the C2C12 mouse myoblast cell line (Bennett and Tonks 1997; Moran et al. 2002) and further supports the conclusion that terminal RD cell differentiation has been observed here.

The TaqMan analysis that has been carried out also verifies that this cell line expresses a number of cholesterol metabolism genes (HMGCR and $L D L R$ ) and clock genes (BMAL1, PER2, CRY1, DBP). Expression levels for most of these genes seem to remain unchanged during the differentiation process. There is, however, a significant decrease in expression levels of HMGCR and $L D L R$ by day 10 , which is similar to the observed decrease in MYOD1 and MYOG expression levels, and could be due to the continued proliferation of these particular cells throughout differentiation (Figure 3-1); by day 10 the cells are becoming overgrown and therefore perhaps starting to die by apoptosis. 
However, in contrast there is no similar decrease observed in expression of MYH3 or DBP. This implies that the decrease could also be due to changes in cell requirements upon differentiation, where differentiated cells need less MYOD1 and MYOG and have a reduced requirement for cholesterol synthesis. The reason for the continued steady increase in $D B P$ expression is as yet unknown but it could be possible that, as a transcription factor, as well as playing a role in the output of the core clock $D B P$ may play an as yet unidentified role in the process of myogenic differentiation.

It is important to recognise that the referred-to differentiation of a population of RD cells will always be limited. As was previously described (section 3.2.1), rhabdomyosarcoma cells are deficient in their ability to exit the cell cycle and complete terminal differentiation, despite their commitment to the muscle cell lineage and expression of the myogenic regulatory factors MYOD1 and MYOG. Several possible explanations for this deficiency have been proposed (Aguanno et al. 1990; Knudsen et al. 1998; Tapscott et al. 1993), including the presence of inactive MYOD1 that is still able to bind DNA but not activate transcription (Tapscott et al. 1993). Several research groups have been able to partially rescue the phenotype of limited RD cell differentiation, demonstrating growth inhibition as well as induction of both partial differentiation and the expression of muscle-specific proteins (Aguanno et al. 1990; Bouche et al. 2000; Wang et al. 2010b), or the restoration of MYOD1 activity (Guo et al. 2003; Ostrovsky et al. 2002; Puri et al. 2000). However, despite all of these treatments and modifications, it is clear that the rescue remains only partial and that resulting cell populations remain mixed, containing RD cells at varying stages of differentiation, making it unclear how beneficial these additional steps are. Within the experimental paradigm used here, it is clear that reliable cell differentiation is being observed, as demonstrated by expression of $\mathrm{MYH}$, even if this differentiation is not complete. Therefore, since any additional approaches have the potential for generating further experimental variability, I will continue to use this cell line, using serum deprivation to initiate cell differentiation, as a starting point for generating the in vitro model for studying statin administration. 
One main reason for the characterisation of the differentiation process was to find the most suitable length of time to use for muscle cell differentiation prior to future experiments in this particular project. RD cells used by other research groups have been left in low-serum differentiation medium for varying lengths of time prior to further experiments, including for 4-5 days (Knudsen et al. 1998), 6 days (Vachon et al. 1996) or 7 days (Nishimoto et al. 2003). From the results obtained, it was decided that cells should be left in low serum differentiation medium for 5 days prior to future experiments. After this time the muscle regulatory factors $M Y O D 1$ and $M Y O G$ are being expressed near their highest level, there is a high level of $M Y H 3$ expression indicating that there are differentiated cells, and expression levels of the cholesterol metabolism genes and clock genes remain stable. Using 5 days for differentiation will also allow enough time to carry out any required time courses before the cells get too overgrown and gene expression levels start to decrease.

\subsubsection{Analysis of Circadian Rhythms}

Having observed these changes in gene expression during RD muscle differentiation, temporal gene expression was then studied in this cell line following a serum pulse. Circadian rhythms have now been observed in most peripheral tissues and also in many human cell lines (Ko and Takahashi 2006), but there have, to date, been no reported studies into circadian rhythmicity in this particular human muscle cell line.

TaqMan analysis of temporal gene expression in both Huh7 (Figure 3-7) and differentiated RD (Figure 3-8) cells indicated that the genes examined were not rhythmic in these cell populations. The lack of observed rhythmicity is of some surprise, especially in light of the fact that rhythms have to date been observed in a large range of different cell lines, using this same serum pulse protocol to synchronise the rhythms of individual cells within a population.

There is also increasing evidence regarding circadian rhythmicity in skeletal muscle (Andrews et al. 2010) and so it might be expected that RD skeletal muscle cells would contain molecular clocks which could be synchronised in culture. On the other hand, it could perhaps be that disruption of circadian 
rhythmicity is a phenotype of the rhabdomyosarcoma tumour from which this cell line is derived. There is currently no evidence for this, and these cells clearly do express a variety of core clock genes; however it is interesting to note that expression of BMAL1 is very low in RD cells, compared to the expression of other clock genes (Figure 3-12). It is unclear from the literature whether BMAL1 is always expressed at a low level in cultured cells or whether this is specific to RD cells. If it is the latter then perhaps this could partially explain the lack of observed rhythmicity, since it is known that deficiency in BMAL1 can lead to arrhythmicity (Bunger et al. 2000). It would also be interesting to know whether or not this low expression is mirrored in the expression of the dimerisation partner of BMAL1, circadian locomotor output cycles kaput (CLOCK).

However, lack of a functional molecular clock in RD cells would not explain the lack of rhythmic gene expression also observed in Huh7 cells (Figure 3-7). Unlike for RD cells, circadian rhythmicity has previously been demonstrated in this cell line ( $\mathrm{Li}$ et al. 2008) and so it could somehow be that there was a problem with the protocol that was used here. This would be supported by the fact that, in the pilot experiment, although rhythmic expression of PER2 and $D B P$ was observed in the 3T3-L1 cell line (Figure 3-6), rhythmic gene expression was not clearly observed in human HepG2 and Huh7 cells. In further support of this, although there were also no clear rhythms in temporal gene expression observed in Huh7 or RD-D cells in the more comprehensive analysis, there were indications of slight rhythmic variations in the expression of $D B P$ in Huh7 cells and BMAL1 in RD-D cells. In Huh7 cells, there was a statistically significant difference between expression of $D B P$ at 16 hours (a potential trough) and 44 hours (a potential peak), and in RD-D cells statistical significance was found between BMAL1 gene expression at 16 hours (a potential peak) and at 32 or 48 hours (2 potential troughs). These temporal variations match the expression profiles seen previously in 3T3-L1 cells (Otway et al. 2009) and HepG2 cells (Koyanagi et al. 2006). In addition, the errors on the data from TaqMan analysis were quite large. All of this could indicate that the serum pulse was only partially achieving its intended goal of cell synchronisation. In order to investigate this possibility, it would have perhaps 
been interesting to see whether either serum starvation prior to serum shock (Tracey et al. 2012) or cell synchronisation using the glucocorticoid hormone analogue dexamethasone rather than horse serum (Balsalobre et al. 2000) would have had any impact on the observation of circadian rhythmicity.

It has been demonstrated that the housekeeping genes that are most commonly used for data normalisation are not always the most appropriate genes (Kamphuis et al. 2005), and that the decision of housekeeping gene should be made in light of each individual experimental situation (Vandesompele et al. 2002). This is perhaps especially important to bear in mind in circadian experiments when a large number of genes, other than the genes of interest, are likely to also exhibit circadian variation. In this particular case, any control gene used would need to be expressed in both liver and muscle, to not vary in a circadian manner and not be affected by the statin treatment which would be a part of later experiments. In this case it was clear that there was large variation in the expression of each of the housekeeping genes used (Figure 3-9), which could potentially complicate the interpretation of data when normalised and so the decision was taken to leave data unnormalised. In addition there was no significant difference to the observation of circadian rhythmicity between unnormalised data or data normalised to either of the two housekeeping genes $18 S$ or $\beta$-actin (Figure 3-10). Therefore, it seems unlikely that the choice of an incorrect housekeeping gene was responsible for the observation of arrhythmicity in this particular case.

It remains possible that circadian rhythmicity may be observable in undifferentiated RD cells where no rhythms were observed here in differentiated cells (Figure 3-8). Alternatively, perhaps protein expression may have shown temporal variation even where RNA did not, since changes in functional protein are not always directly related to changes at the RNA level (Brockmann et al. 2007; Greenbaum et al. 2003; Griffin et al. 2002). However, given the lack of evidence for rhythmicity in gene expression that has been observed so far, a decision was made not to commit further resources to continue investigating this line of research. 


\subsubsection{New Aims and Objectives:}

Therefore, having characterised the differentiation process of RD muscle cells (objective 1; section 1.5) but having been unable to verify the ability of this cell line to establish and maintain a circadian cycle in vitro (objective 2, section 1.5), a new set of objectives needed to be decided upon. Having established human liver and muscle models, I shall continue to compare the responses of liver and muscle cells to statin treatment, now focusing on investigating differences in the effects on cell viability, amount of cellular cholesterol and on prenylation, which may give us further clues into the mechanism of statin-induced muscle toxicity.

The new aim is to use liver and muscle cells in culture as a model system for studying the effects of statin administration on both cholesterol and isoprenoid biosynthesis, via the following objectives:

Objective 2: Examine the effects of statins on cell viability to determine whether there are differences between liver and muscle.

Objective 3: Assess the relative expression of HMGCR, and changes in cellular cholesterol in response to statin treatment, in liver and muscle.

Objective 4: Assess whether differences measured in objectives 2 and 3 correspond to effects of statin treatment on protein prenylation. 


\subsection{Summary}

Circadian rhythms have been shown to impact upon the toxicity of xenobiotics. To examine whether differences exist between the circadian aspects of statin efficacy and toxicity the temporal affects of statins can be studied using an in vitro model. In order to compare the responses of liver (the target tissue) and skeletal muscle (the site of toxicity) to temporal statin administration two human cell lines were chosen: RD human embryonic rhabdomyosarcoma cells, which express a number of muscle-specific genes following differentiation, and HepG2 human hepatocellular carcinoma cells.

The morphological and molecular changes that occurred during RD muscle cell differentiation were characterised. RD differentiation was observed in response to a change to low-serum medium, as determined by large increases in the expression of muscle-specific transcription factor MYOG and the marker of terminal differentiation $M Y H 3$. Levels of $M Y H 3$ rose rapidly after 4 days in low serum medium and remained high throughout the remainder of the 10-day experiment, whereas levels of MYOG began to decline after 8-10 days. As a result of this analysis, it was decided to use a time of 5 days for the differentiation of RD cells in future experiments.

$\mathrm{RD}$ cells were also observed to express a number of important cholesterolrelated and clock genes. However, significant rhythmic gene expression was not observed in response to a serum pulse, in either liver or muscle cells, but was observed in the positive control 3T3-L1 cell line. Therefore, a new set of objectives were defined, whereby the focus of future experiments would remain on examining the differences in the response of liver and muscle to statin administration, but without the circadian aspect. 


\section{Differential Sensitivity of Liver and Muscle Cell Lines to Statin Treatment}

\subsection{Introduction}

Statins act by inhibiting 3-hydroxy-3-methylglutaryl coenzyme A reductase (HMGCR), the enzyme that catalyzes the rate-limiting step in cholesterol synthesis (Shitara and Sugiyama 2006). Decreased de novo cholesterol synthesis leads to an upregulation of the LDL receptor, increased uptake of lowdensity lipoprotein (LDL) from the bloodstream and an overall reduction in patient plasma levels of cholesterol in the form of LDL (Brown and Goldstein 2004). As patient plasma LDL-cholesterol is a direct marker of cardiovascular risk, the action of the statins reduces risk of cardiovascular disease in patients (Mensink et al. 2003). The extent of the reduction in LDL is dependent on the specific statin used in treatment and on the dose (Chong et al. 2001).

There are a number of different statins currently clinically available, including simvastatin, lovastatin, fluvastatin, atorvastatin, rosuvastatin and pravastatin. All statins exert their effects via the same mechanism (Chong et al. 2001); however, they have differences in their physicochemical properties (Table 1-3). These include differences in their chemical structure (Figure 1-4), their bioavailability, half-life, potency, lipophilicity, metabolism and transport (Chong et al. 2001; Shitara and Sugiyama 2006; Vaklavas et al. 2009). In addition, both simvastatin and lovastatin are administered as a prodrug, containing an inactive lactone ring, which is converted into the biologically active open acid form in the body. All other statins are given in the open acid form (Chong et al. 2001; Shitara and Sugiyama 2006).

Myopathy, both in general and in its severest form as rhabdomyolysis, has been observed as an adverse effect with all statins (Bolego et al. 2002), although there are marked differences in myopathy incidence rates (Staffa et al. 2002). The fact that cerivastatin was withdrawn from the worldwide market in 2001 due to its high incidence of cases of fatal rhabdomyolysis (Bellingham 2001) highlights the significance of the problem of statin-induced muscle toxicity. 
However, the exact mechanism of this greater muscle sensitivity has not been fully elucidated (Baker 2005).

In the previous chapter, the muscle cell line RD was characterised in terms of its differentiation, for use together with the control liver cell line Huh7 as part of a model system. The next stage was to begin characterising the responses of these liver and muscle cell lines to statin treatment, in terms of overall cell toxicity and cholesterol metabolism. Cell toxicity assays have been carried out, in order to test the hypothesis that greater toxicity would be observed in the RD muscle cells than in Huh liver cells, since muscle toxicity is an important adverse effect of statins in the clinic. This study represents the first comprehensive comparative analysis of statin toxicity in both liver and muscle cell lines.

In addition, the expression of HMGCR has been compared between cell lines, and the effect of statin treatment on the level of total cellular cholesterol examined, in order to investigate whether any differences observed in toxicity translate to differences in statin-induced cholesterol reduction.

\subsection{Characterisation of Statin-Mediated Cell Toxicity}

In order to begin characterising the effects of statin treatment in RD and Huh7 cells, MTT assays were carried out to assess metabolic and cellular viability after cells were exposed to different concentrations of statin for 48 hours (section 2.2.1.12). 
Huh7 cells have been used previously for MTT assays in this research group, using a period of 150 minutes for the incubation of cells with MTT reagent (Mary Hong, personal communication, 10 November 2009). However, RD cells have not previously been used by this group, and therefore a preliminary experiment was carried out using untreated cells in order to determine optimum assay conditions prior to statin treatment (Figure 4-1).

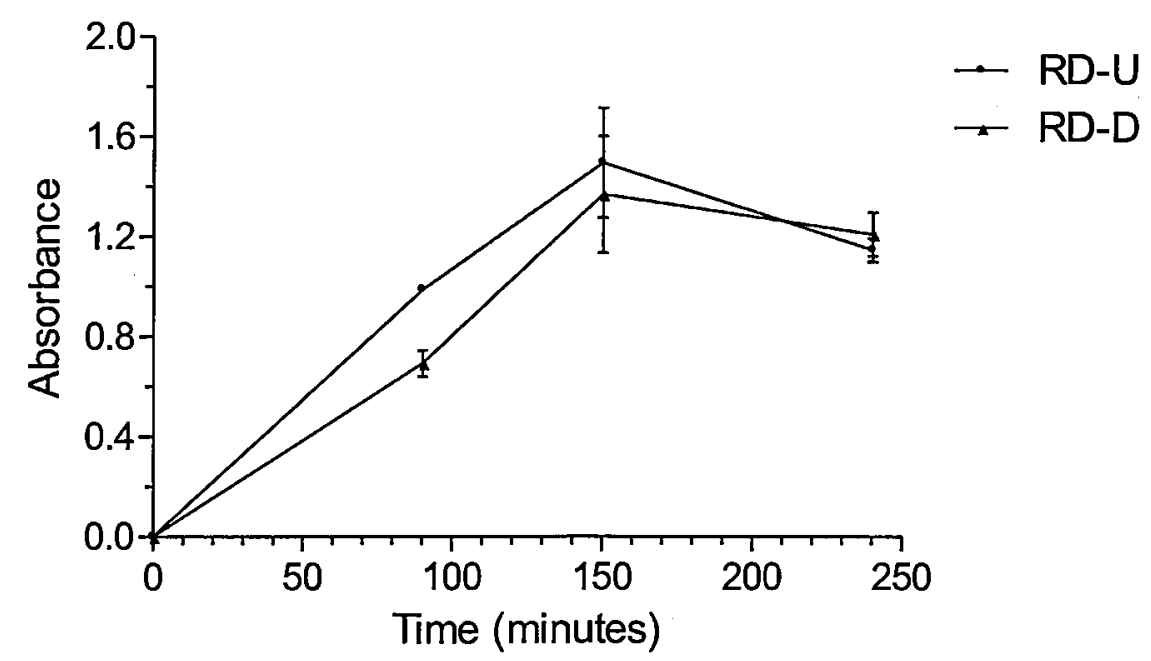

Figure 4-1 Optimisation of time of incubation with MTT reagent

MTT reagent was added to untreated RD-U and RD-D cells in 6-well plates $(T=0)$ and absorbance was read (section 2.2.1.12) after incubation for 90,150 and 240 minutes. Different wells of cells were used for each time point. Values plotted represent mean \pm standard deviation (SD), from a single experiment where $N=3$.

One hundred and fifty minutes was found to be the minimum time at which absorbance no longer increased linearly with incubation time, using both undifferentiated RD (RD-U) and differentiated RD (RD-D) cells. This would therefore allow sufficient time for the conversion of MTT reagent by viable cells, and was chosen for incubation of all cells with MTT reagent in following assays.

Subsequently, MTT assays were carried out using the full range of available statins: simvastatin (acid and lactone), lovastatin (acid and lactone), cerivastatin, fluvastatin, atorvastatin, rosuvastatin and pravastatin (Figure 4-2). 

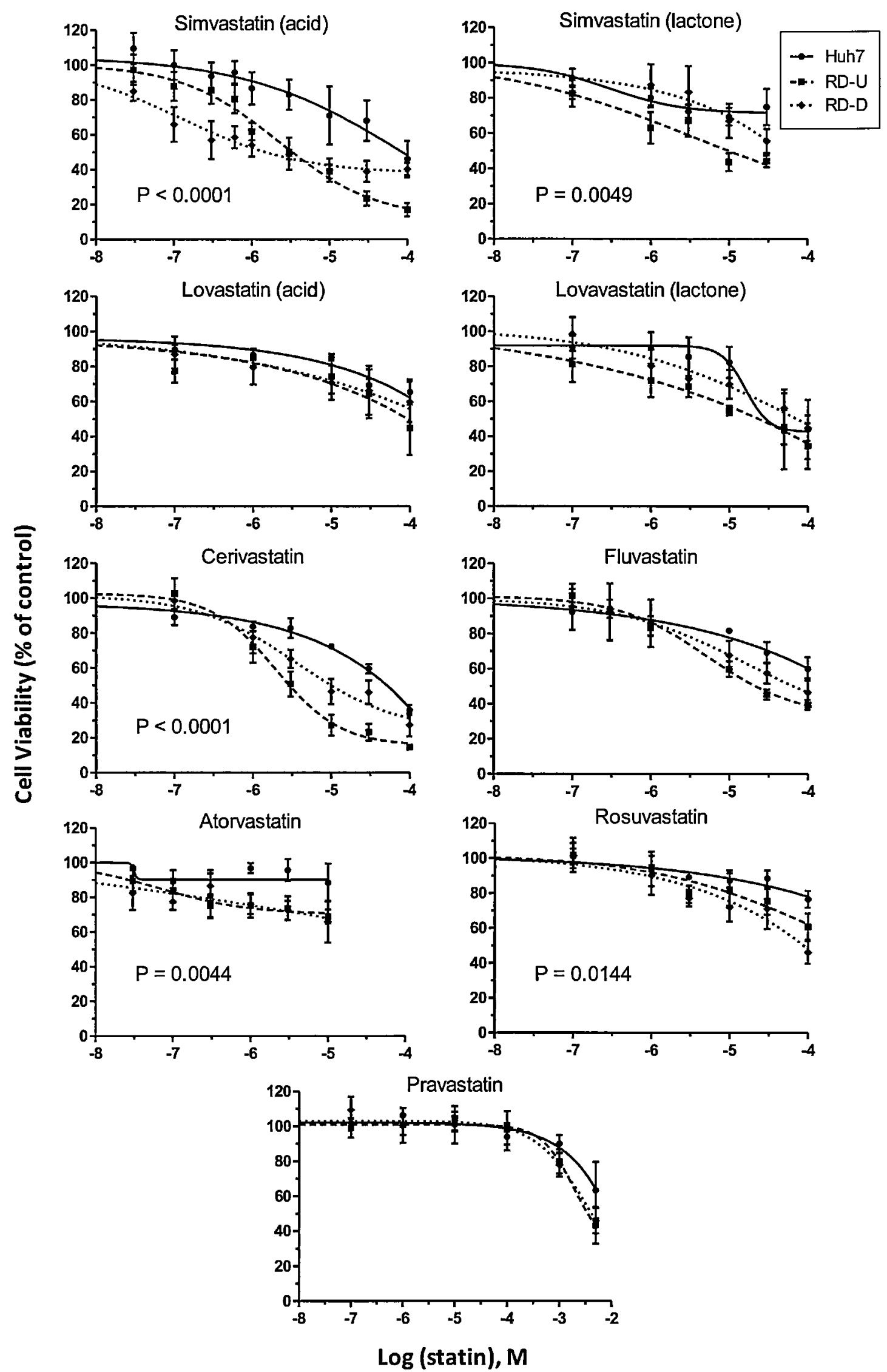

Figure 4-2 Cell viability in response to statin treatment

Huh7 (shown in red), RD-U (blue) and RD-D (black) cells were treated with statin for 48 hours and MTT assays carried out to measure cell viability. Graphs are shown in decreasing order of statin lipophilicity. Final absorbance is expressed as a percentage of the control (defined as $100 \%$ ). At least three independent experiments were carried out, using 6 replicate wells in each. Mean values were generated from each experiment, and combined to produce the data shown here. Error bars represent standard error of the mean (SEM). Data were analysed by two-way analysis of variance (ANOVA) and $P$ values are shown where the difference between resoonses of different cell lines was determined to be statisticallv sionificant $(\mathrm{P}<0.05)$. 
There was a significant dose-dependent reduction in cell viability, in all cell types, on treatment with all statins $(P=0.0041$, atorvastatin; $P<0.0001$, all other statins, two-way analysis of variance (ANOVA)). The largest reductions in cell viability were observed on treatment with lipophilic simvastatin (acid) and cerivastatin, whereas loss of cell viability was only observed with very high concentrations of the hydrophilic pravastatin ( $1 \mathrm{mM}$ and greater).

It was not possible to determine robust IC50 values for all of the statins in all cell types, since full dose-response curves could not always be obtained, due to limits in statin solubility and the need to not exceed $0.1 \%$ DMSO in cell medium. However, where IC50 values could be determined, they were consistent with other values found in the literature (Table 4-1).

\begin{tabular}{|c|c|cc|c|}
\cline { 2 - 5 } \multicolumn{1}{c|}{} & \multicolumn{3}{c|}{ IC50 $(\boldsymbol{\mu M})$} \\
\hline Statin & Huh7 & RD-U & $\begin{array}{c}\text { (Kobayashi } \\
\text { 2008) }\end{array}$ & RD-D \\
\hline Simvastatin (acid) & 102.2 & $2.36 \pm 0.39$ & 3.99 & $0.296 \pm 0.20$ \\
\hline Simvastatin (lactone) & $0.27 \pm 0.10$ & $3.60 \pm 5.27$ & - & \\
\hline Lovastatin (acid) & & & 52.2 & \\
\hline Lovastatin (lactone) & 15.98 & & - & \\
\hline Cerivastatin & & $2.06 \pm 0.48$ & 1.77 & $4.437 \pm 1.90$ \\
\hline Fluvastatin & $8.51 \pm 5.49$ & $6.02 \pm 1.83$ & 8.34 & $2.624 \pm 2.56$ \\
\hline Atorvastatin & & & 9.26 & \\
\hline Rosuvastatin & & 160.9 & $>100$ & \\
\hline Pravastatin & & & 4890 & \\
\hline
\end{tabular}

Table 4-1 IC50 values for the reduction in cellular viability on statin treatment

Values are shown as mean \pm SEM, where possible, or otherwise as a single value, determined from the relevant graph (shown in Figure 4-2) using GraphPad Prism version 5 (section 2.2.5). Not all IC50 values could be determined from this data. IC50 values taken from Kobayashi et al. 2008, as determined by MTT assay using RD-U cells, are also given for comparison. Kobayashi et al. 2008 used only the acid forms of simvastatin and lovastatin, as prepared from their lactone forms prior to treatment. 
In general, greater cell toxicity for a given concentration of statin was observed in the RD cells, either differentiated or undifferentiated, compared to the Huh7 cells. The extent of this difference was dependent on the statin and the overall difference in responses between cell lines was only determined to be statistically significant with simvastatin $(P<0.0001$, acid form and $P=0.0049$, lactone, two-way ANOVA), cerivastatin ( $P<0.0001)$, atorvastatin $(P=0.0044)$ and rosuvastatin $(P=0.0144)$ (Table $4-2)$. The difference between toxicity in the different cell types was found to be of greater significance with lipophilic simvastatin and cerivastatin than with hydrophilic rosuvastatin.

\begin{tabular}{|c|c|c|}
\hline Statin & P value summary & P value \\
\hline Simvastatin (acid) & $* * * *$ & $<0.0001$ \\
\hline Simvastatin (lactone) & $* *$ & 0.0049 \\
\hline Lovastatin (acid) & $\mathrm{ns}$ & 0.1137 \\
\hline Lovastatin (lactone) & $\mathrm{ns}$ & 0.1128 \\
\hline Cerivastatin & $* * * *$ & $<0.0001$ \\
\hline Fluvastatin & $\mathrm{ns}$ & 0.3394 \\
\hline Atorvastatin & $* *$ & 0.0044 \\
\hline Rosuvastatin & $*$ & 0.0144 \\
\hline Pravastatin & $\mathrm{ns}$ & 0.6118 \\
\hline
\end{tabular}

Table 4-2 Significance of cell type in determining response to statin treatment

Data from MTT assays (Figure 4-2) were analysed by two-way analysis of variance (ANOVA) to determine whether there was a significant difference between the responses of different cell types to statin treatment. Ns $=$ not significant, ${ }^{*}=\mathrm{P}<0.05,{ }^{* \star}=\mathrm{P}<0.01,{ }^{* * *}=\mathrm{P}<0.0001$.

It is also interesting to note that there appears to be a difference in the cell viability responses of RD-U and RD-D cells to statin treatment. Not all of the relevant IC50 values could be determined, making a quantitative comparison difficult. However, Bonferroni post hoc analysis suggested that these differences were not statistically significant $(P>0.05)$.

In summary, these data demonstrate effects of cell type and of different statins on cell toxicity in response to statin treatment. 


\subsection{Analysis of Cholesterol Metabolism in Liver and Muscle Cells}

Having characterised cell viability in response to statin treatment it was important to also examine cholesterol metabolism in these different cell lines, to see whether there were any differences that could account for the differences in cell toxicity.

\subsubsection{HMGCR Expression}

Since HMGCR, the enzyme that catalyses the rate-limiting step in cholesterol biosynthesis, is the target of the statins (section 4.1), an initial step in characterising cholesterol metabolism in RD and Huh7 cells was to examine the relative abundance of this enzyme.

Analysis of HMGCR gene expression in cells growing in normal conditions revealed a statistically significant difference between transcript levels of HMGCR in RD and Huh7 cells (Figure 4-3). HMGCR transcript levels were 1.5 fold lower in RD-U cells than Huh7 cells ( $P<0.05$, Bonferroni post hoc), and 3fold lower in RD-D cells than Huh7 cells $(P<0.001)$.

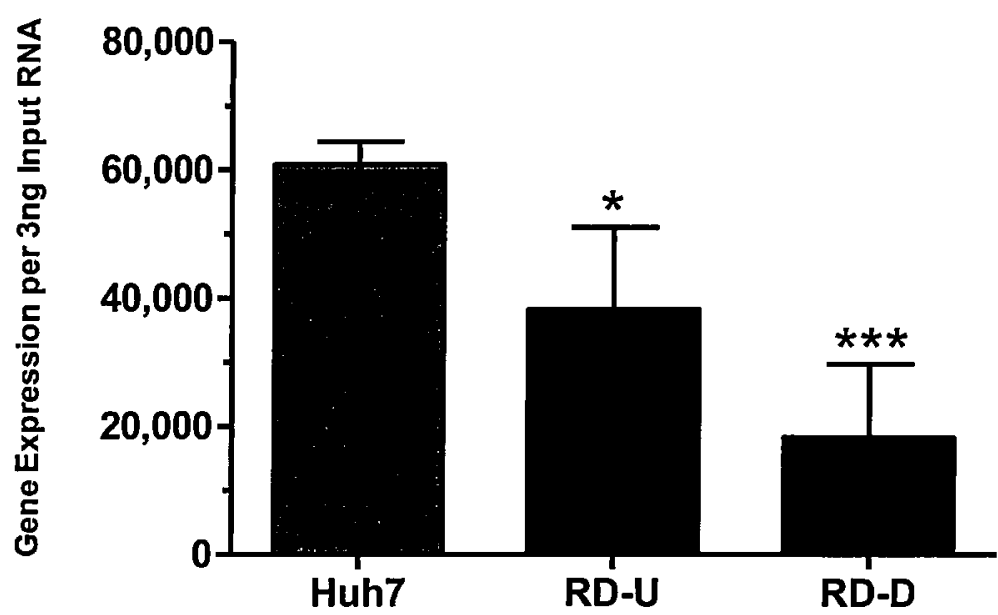

Figure 4-3 HMGCR gene expression in different cell types

Total RNA was extracted from Huh7, RD-U and RD-D cells in standard growth conditions and expression of HMGCR was measured by quantitative RT-PCR (TaqMan). Data are presented as mean \pm standard deviation (SD), from a single experiment where $N=4$. Statistical significance was determined by one-way ANOVA followed by Bonferroni post hoc test, ${ }^{*}=\mathrm{P}<$ $0.05,{ }^{* * *}=\mathrm{P}<0.001$ compared to Huh7 expression. 
Western blot analysis of total protein showed that HMGCR protein expression was similarly found to be considerably lower in RD-U than Huh7 cells (Figure 4-4). HMGCR protein levels were even lower in RD-D cells, as evidenced by the need to increase the exposure time in order to detect HMGCR in this cell type. Densitometry suggested that there was a 6-fold difference in protein expression between Huh7 and RD-U cells however densitometry was not possible on protein levels in RD-D cells due to the large difference in signal intensity between the cell types.

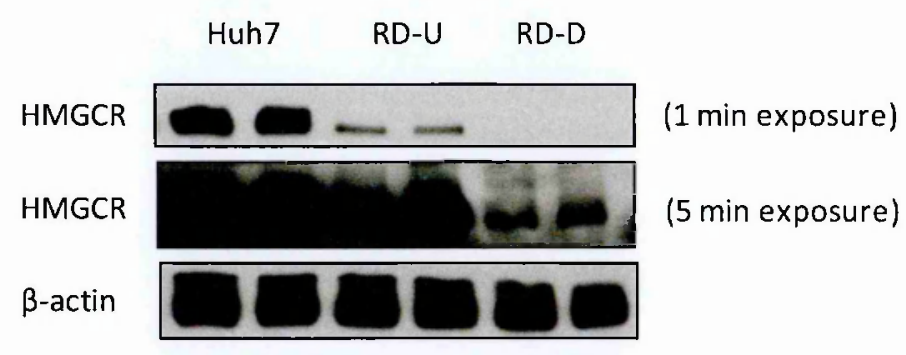

Figure 4-4 HMGCR protein expression in different cell types

Total protein was extracted from Huh7, RD-U and RD-D cells in standard growth conditions and $15 \mathrm{\mu g}$ was separated by SDS-PAGE. The level of HMGCR (90kDa) was detected by western blot and $\beta$-actin was used as a loading control. Two different exposure times were used to allow detection of HMGCR in all cell types. $N=2$.

These data demonstrate that HMGCR is expressed in all cell types of interest: Huh7, RD-U and RD-D cells. At both the transcript and the protein level, there is a much greater expression of this enzyme in Huh7 liver cells than in RD muscle cells. Interestingly, a difference was also observed between HMGCR expression in RD-U and RD-D cells, with lower expression in RD-D cells, particularly at the protein level. The different levels of HMGCR expression between the cell types may need to be taken into account when interpreting results from future experiments. 


\subsubsection{Total Cellular Cholesterol}

Having verified the presence of HMGCR in all cell types, the next stage was to determine what effect statin treatment would have on total cellular cholesterol content in these cells, since reduction of cholesterol is the main aim of statin use in the clinic.

Assays were carried out in which cells were treated with statin for a 48 hour period, after which cholesterol was extracted and quantified (section 2.2.4). This time period was chosen based on those reported in the literature (Kobayashi et al. 2008; Mullen et al. 2010), and to remain consistent with the conditions used for the previous MTT assays (Figure 4-2). Simvastatin (acid), cerivastatin and pravastatin were chosen for use in this analysis since both simvastatin and cerivastatin showed a statistically significant difference in toxicity between RD muscle and Huh7 liver cells (Figure 4-2). In contrast, pravastatin treatment resulted in toxicity only at high concentrations and showed no statistically significant difference in toxicity between cell lines, and was included as a relatively non-toxic comparator.

Initially, cells were treated with $1 \mu \mathrm{M}$ of each of the chosen statins. This concentration was chosen based on the work of Kobayashi and colleagues (Kobayashi et al. 2008), and results from the MTT assays: In these MTT assays, treatment with $1 \mu \mathrm{M}$ simvastatin and cerivastatin resulted in differences between the responses of RD muscle and Huh7 liver cells without causing an excess of cell death that could complicate extracting sufficient cholesterol for quantification (Figure 4-2). Where appropriate, the statistical significance of statin treatment on total cellular cholesterol in individual cell lines was determined using unpaired two-tailed t-test. 
There appeared to be a decrease in total cellular cholesterol in Huh7, RD-U and RD-D cells upon $1 \mu \mathrm{M}$ simvastatin treatment, and in Huh7 and RD-D cells on $1 \mu \mathrm{M}$ cerivastatin treatment (Figure 4-5). However, where statistical analysis was appropriate, these changes were not found to be significant using unpaired t-tests; $(P=0.2262$ (Huh7) and $0.0574(\mathrm{RD}-\mathrm{U})$ on treatment with $1 \mu \mathrm{M}$ simvastatin.) There appeared to be no change in cholesterol level upon RD-U treatment with cerivastatin or in any of the cell types on pravastatin treatment.

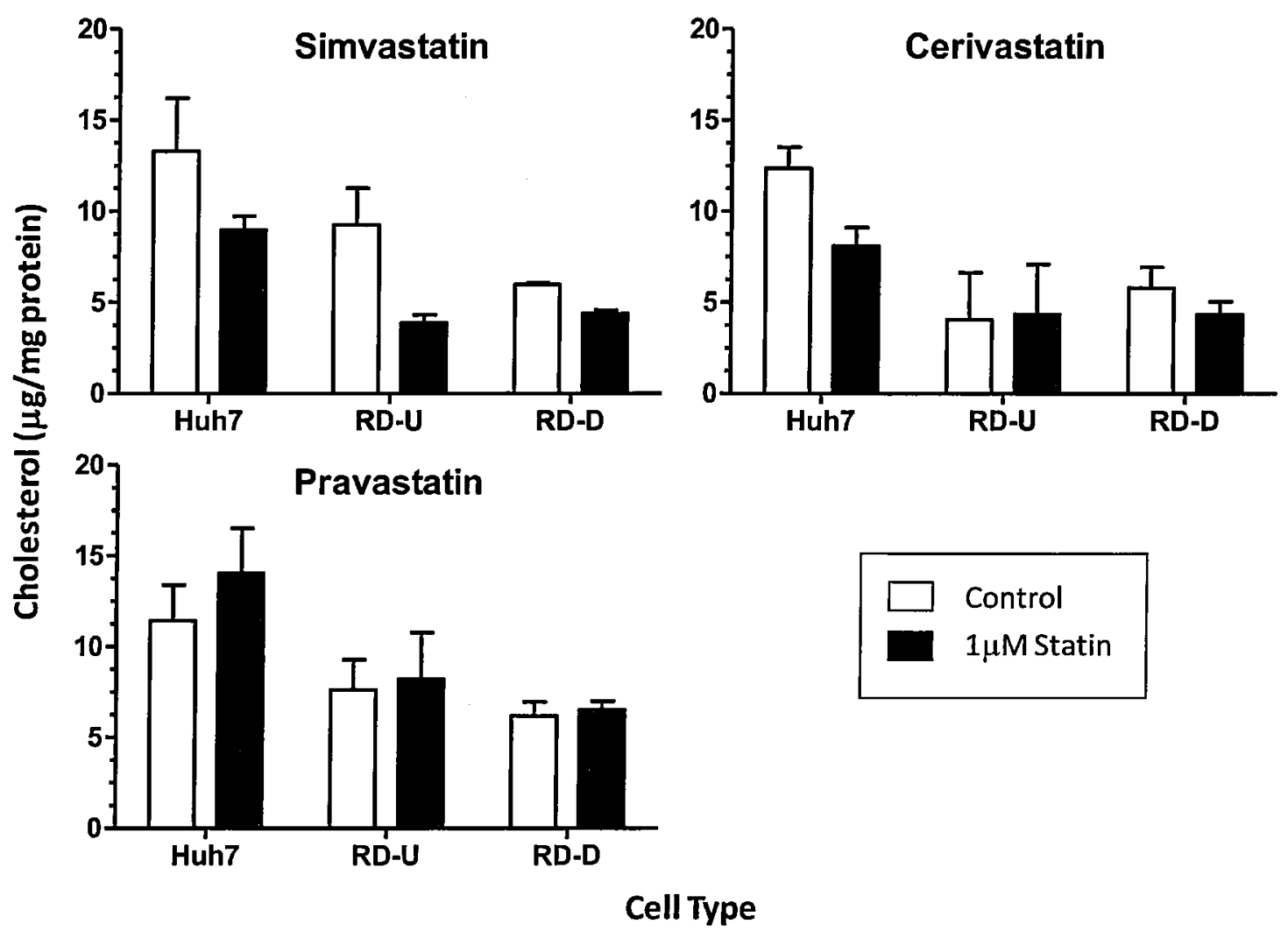

Figure 4-5 Effect of $1 \mu \mathrm{M}$ statin treatment on total cellular cholesterol

Huh7, RD-U and RD-D cells were treated with $1 \mu \mathrm{M}$ simvastatin (acid), cerivastatin or pravastatin for 48 hours, after which membrane cholesterol was extracted using chloroform: methanol, quantified using the Amplex Red Cholesterol Assay Kit and normalised to total protein. DMSO was used as a vehicle control for simvastatin and water for cerivastatin and pravastatin. Values plotted represent mean \pm SEM from two (cerivastatin and pravastatin) or three (simvastatin) independent experiments, where there were 3 replicates in each experiment. 
Since no change in cholesterol was observed on treatment with $1 \mu \mathrm{M}$ pravastatin, and changes with simvastatin treatment were not found to be statistically significant, the same analysis was then carried out after treating cells with $10 \mu \mathrm{M}$ simvastatin and pravastatin. Data is not shown for the use of $10 \mu \mathrm{M}$ cerivastatin since at this concentration the amount of cell death, particularly in the RD-U cells, was so great that it was not possible to extract sufficient cholesterol from the remaining cells as to enable quantification.

Treatment with $10 \mu \mathrm{M}$ of both simvastatin and pravastatin seemed to result in a decrease in total cellular cholesterol in Huh7 cells $(P=0.00535$ and 0.2144 respectively). A statistically significant cholesterol decrease was observed in RD-D cells on treatment with both simvastatin $(P=0.0027)$ and pravastatin $(P=$ 0.0023) (Figure 4-6). Therefore, since these are the concentrations that

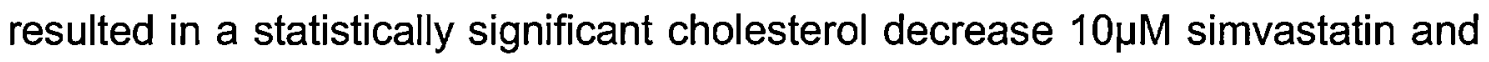
pravastatin were chosen for use, alongside $1 \mu \mathrm{M}$ cerivastatin, in future experiments.
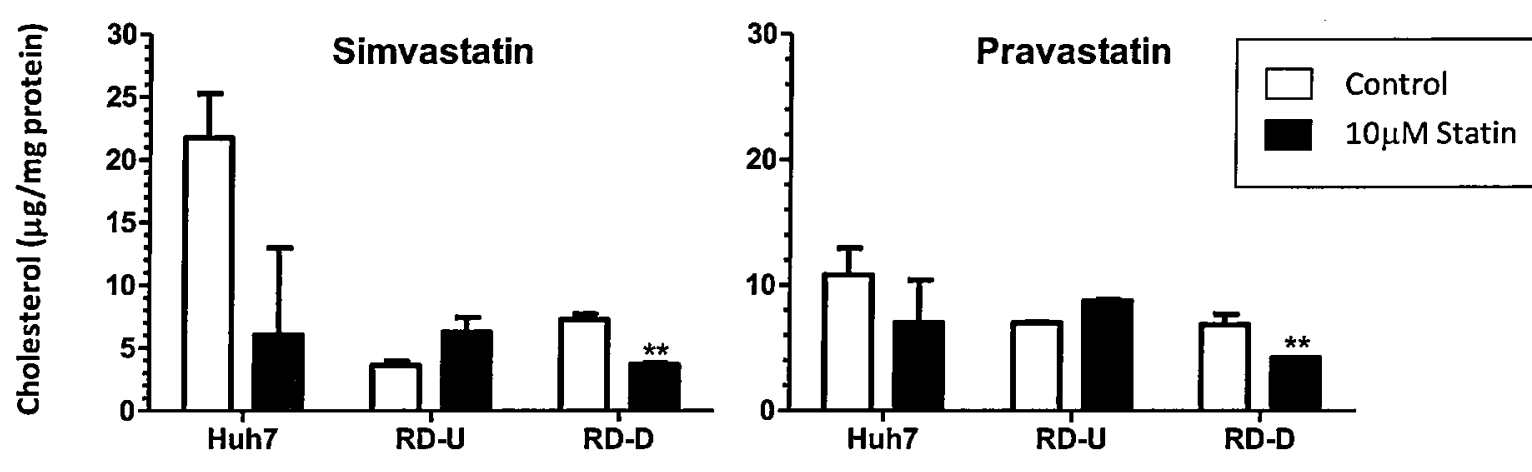

Cell Type

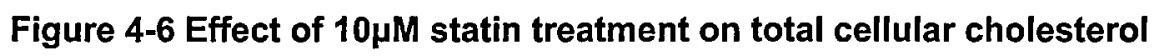

Huh7, RD-U and RD-D cells were treated with 10 $\mu \mathrm{M}$ simvastatin (acid) or pravastatin for 48 hours, after which membrane cholesterol was extracted, quantified and normalised to total protein. Values plotted represent median \pm range; from a single experiment where $N=3$. Statistical significance determined by unpaired $t$-test, ${ }^{\star \star}=P<0.01$ compared to relevant control.

There appeared to be some variability with this method of cholesterol extraction and quantification. This is particularly apparent when comparing the level of cholesterol between control treatments, for example cellular cholesterol ranged from $10.6-20.1 \mu \mathrm{g} / \mathrm{mg}$ protein in control Huh7 cells and from $3.6-9.3 \mu \mathrm{g} / \mathrm{mg}$ 
protein in control RD-U cells (Figure 4-5 and Figure 4-6). Therefore, experiments were carried out in order to investigate the source of this variation.

In order to assess whether the vehicle control used could have had an effect on level of cholesterol measured Huh7 cells were seeded into 6-well plates and T25 flasks and treated with a range of different controls (Figure 4-7). Controls used were water (control for cerivastatin and pravastatin), $0.1 \%$ DMSO (simvastatin control) and $1 \%$ DMSO. A medium-only control was not included here since the water-containing control was considered equivalent to this. The effect of exclusion of non-essential amino acids (NEAA) from Huh7 cell medium was also examined. No statistically significant difference was found between control treatments $(P=0.2838$ (6-well plates), $P=0.1447$ ( $T 25$ flasks), one-way ANOVA).
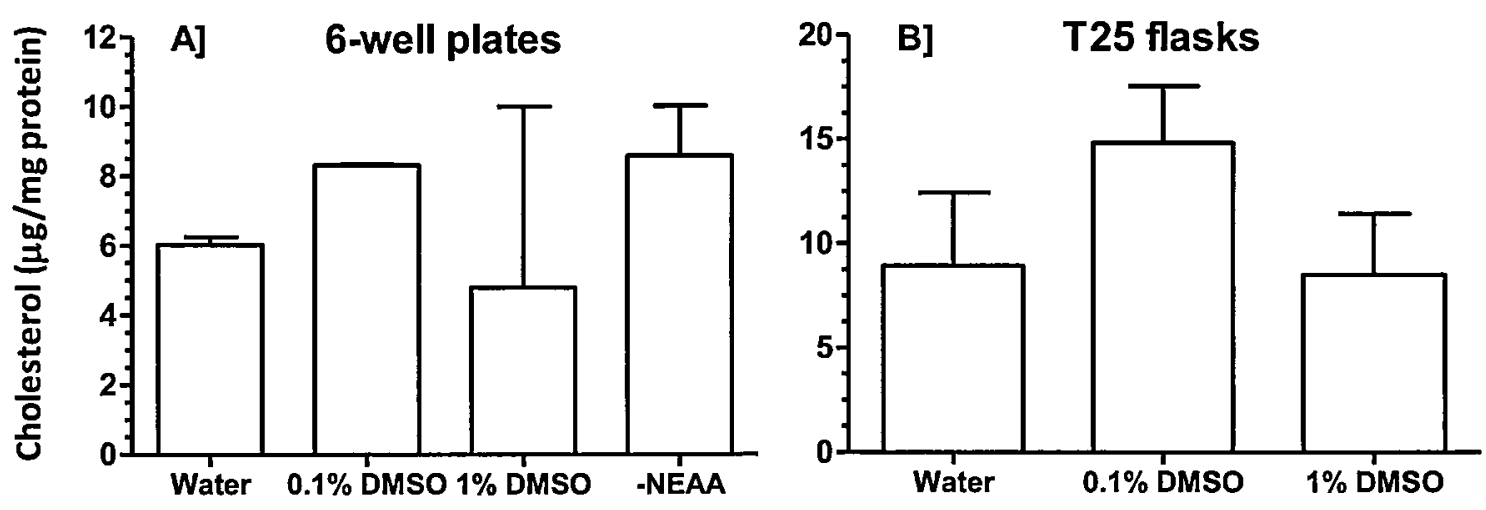

Treatment

Figure 4-7 Effect of control treatment on total cellular cholesterol in Huh7 cells Huh7 cells in 6 -well plates (A) or T25 flasks (B) were treated with $0.1 \%$ DMSO, water, $1 \%$ DMSO, or serum-free media without the addition of non-essential amino acids (NEAA) for 48 hours, after which membrane cholesterol was extracted, quantified and normalised to total protein. Values plotted represent median \pm range; from a single experiment where $\mathrm{N}=3$. 
In previous experiments, cholesterol content was normalised to total cellular protein, to take cell number into account, but this may not allow for all effects of variation in cell density. However, no significant difference was observed between cholesterol content of Huh7 cells seeded in T25 flasks at two different densities ( $P=0.9442$, unpaired $t$-test). A 3rd, lower, seeding density appeared to lead to similar normalised cholesterol content however statistical analysis could not be carried out due to insufficiency of data (Figure 4-8).

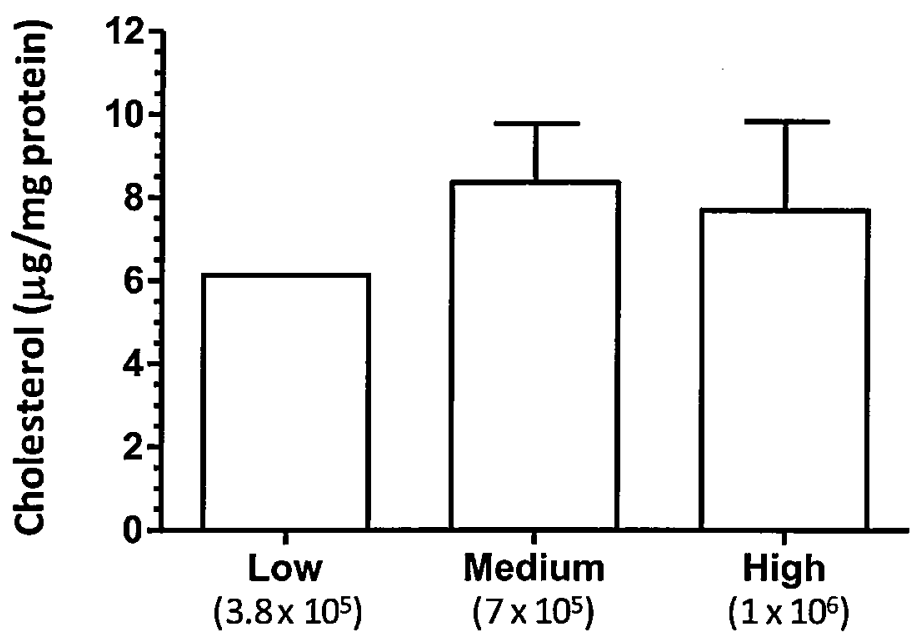

\section{Seeding Density (cells per flask)}

Figure 4-8 Effect of seeding density on total cellular cholesterol in Huh7 cells

Huh7 cells were seeded in T25 flasks using a range of seeding densities (as shown) and treated with $0.1 \%$ DMSO for 48 hours, after which membrane cholesterol was extracted, quantified and normalised to total protein. Values plotted represent median \pm range from a single experiment where $\mathrm{N}=3$ (medium and high densities), or a single value (low density) where sufficient values could not be obtained.

In summary, these data clearly show that that there is a cholesterol pool in both of our model cell lines, Huh7 and RD, and that statin treatment appears to decrease the total cellular cholesterol level. However, variation with the method of cholesterol quantification prevented statistical significance being achieved, with the cause of this variability undetermined. 


\subsection{Discussion}

Myopathy is a well known adverse effect of statin treatment in some patients, with effects ranging from mild to fatal. Importantly, the mechanism of this muscle toxicity remains unclear, making it difficult to minimise the risk of toxicity. In order to gain further understanding, this study first compared statin-mediated cell toxicity in Huh7 liver and RD muscle cells, to test the hypothesis that RD muscle cells would show greater susceptibility to the toxic effects of statins. This represented the first comprehensive comparative analysis of statin toxicity in both liver and muscle cell lines. Subsequently, since HMGCR is the enzyme inhibited by the statins, expression of this enzyme was compared between cell lines at both transcript and protein levels. Finally, cellular cholesterol content in response to statin treatment was also quantified, in order to further examine differences in the responses of these two cell lines.

\subsubsection{Cell Viability}

MTT assays are widely used for assessing the viability of cells (Saad et al. 2006). They rely on the reduction of a tetrazolium bromide salt to a waterinsoluble formazan salt by dehydrogenase enzymes in active mitochondria of living cells (Mosmann 1983). The assay therefore provides a measure of the metabolic activity of cells and does not assess other parameters of cell growth, such as cell division. However, they remain very useful for giving an indication of cell viability in response to xenobiotics and they have been used here to assess cell viability in response to treatment of both Huh7 and RD cells with a range of statins (section 4.2).

\subsubsection{Differences between Liver and Muscle}

The results obtained here showed that there was greater toxicity in RD muscle than Huh7 liver cells in response to treatment with all statins, with statistical significance between the responses on treatment with simvastatin, cerivastatin, atorvastatin and rosuvastatin. This is consistent with the observation of skeletal muscle toxicity as a well known adverse effect of the statins in the clinic (Hedenmalm et al. 2010), and confirms the hypothesis as well as further supporting the relevance of this model system for the study of the effects of statins. 
In addition to a difference between sensitivity of liver- and muscle-derived cells to the toxic effects of statin exposure, treatment appeared to result in greater cell toxicity in RD-U compared to RD-D cells, with statins other than atorvastatin, rosuvastatin and pravastatin. These data are consistent with the recent finding that differentiated $\mathrm{C} 2 \mathrm{C} 12$ mouse skeletal muscle cells are more resistance to cell death by apoptosis than undifferentiated cells, due to an elevation of anti-apoptotic proteins (Xiao et al. 2011). From the data in this study not all IC50 values could be obtained, due to technological considerations, and this prevented a quantitative comparison in some cases. However, Bonferroni post hoc analysis suggests that the differences between RD-U and RD-D responses were not statistically significant, but further work is required to fully examine this finding in a robust manner.

\subsubsection{The Effect of Different Statins and the Role of Statin Exposure}

The results of these MTT assays reveal a difference in toxicity between not only liver and muscle cells, but also between treatment with different statins, with more lipophilic statins such as simvastatin, lovastatin and cerivastatin showing the greatest toxicity, particularly in muscle cells. This is consistent with the observation of the highest incidence of myopathy with simvastatin, lovastatin and cerivastatin treatment in patients (Staffa et al. 2002).

In general, the exposure is thought to play a major role in determining the toxicity of a drug (Caldwell et al. 1995; Hedenmalm et al. 2010), with systemic exposure to xenobiotics determined by a complex interaction of absorption, distribution, metabolism and excretion (ADME) (Caldwell et al. 1995). With the statins, this role of drug exposure in toxicity is supported by the fact that pharmacokinetic interactions that increase systemic and skeletal muscle statin exposure, such as concomitant treatment of a patient with a statin and a xenobiotic that affects metabolism by cytochrome P450 (CYP) enzymes, have been shown to increase myopathy incidence (Bolego et al. 2002; Law and Rudnicka 2006; Mastaglia and Needham 2012; Sidaway et al. 2009). In addition, genetic polymorphisms in the gene encoding organic anion transporting polypeptide 1B1 (OATP1B1), a transporter involved in the hepatic uptake of many of the statins, has been associated with a higher risk of 
myopathy (Link et al. 2008). A correlation has previously been shown between accumulation of statins and cytotoxicity in vitro in RD-U cells, with greater subcellular accumulation of the lipophilic statins being reported (Kobayashi et al. 2008).

The liver is the target organ of the statins, and it has been suggested that nonspecific distribution into other tissues, via passive diffusion, by the more lipophilic statins could explain their increased potential for statin-related muscle problems (Vaklavas et al. 2009). However, myopathy has also been reported with hydrophilic pravastatin, which is not subject to significant passive diffusion, arguing against this hypothesis. It is now known that there are a number of transporters which are expressed in muscle tissue and recognise statins as substrates, and these are more likely to be responsible for uptake of statins into muscle cells (Shitara and Sugiyama 2006). Examples include organic anion transporter 3 (OAT3) (Takeda et al. 2004) and OATP1A4 (Sakamoto et al. 2008) which have been shown to mediate pravastatin transport, and OATP2B1, which is expressed in both liver and muscle and can transport atorvastatin and rosuvastatin (Knauer et al. 2010) as well as pravastatin (Sakamoto et al. 2008).

It would be of interest to determine the transporter profile of the RD cell line and how this compares to skeletal muscle tissue in vivo. It has been shown that the level of expression of transporters, as well as drug metabolising enzymes, can at times be different between cultured cell lines and native tissue (Hilgendorf et al. 2007) and this could have implications for drug distribution. It has also been suggested that differences in transporter profile could partially explain why pravastatin has been shown to have limited effects in some in vitro systems of rat origin, when it clearly can lead to muscle toxicity in human patients (Sakamoto et al. 2008). However, the transporter profile in the human RD muscle cell line is not currently known and so it cannot be certain whether this is also the case in this model system. It is true that the MTT assays did reveal a lesser toxic effect with pravastatin than many of the other statins, but, as already discussed, this does also reflect differences in myopathy incidence observed between statins in the clinic and so the use of pravastatin in this study will be continued. 
Differences in statin cellular exposure are likely to play some role in statininduced muscle toxicity, however they probably do not completely account for differences in the sensitivity between tissues. One recent rat study demonstrated that in vivo there was no difference in systemic or skeletal muscle statin exposure before or during myopathy, and that there was no difference in exposure between different animals and different muscle types that exhibited different sensitivity to muscle toxicity (Sidaway et al. 2009). Since responses of RD and Huh7 cells to statin treatment have been characterised here in terms of gross changes in cellular viability, it is now important to look in more detail at molecular changes occurring in these cell lines in response to statins for further elucidation.

\subsubsection{Cholesterol Metabolism}

\subsubsection{Differences in HMGCR Expression}

These analyses have shown that HMGCR, the enzyme that catalyses the ratelimiting step in cholesterol biosynthesis and is inhibited by the statins (Shitara and Sugiyama 2006), is present in all cell types. This was important to establish prior to further investigations into the mechanisms of statin-induced muscle toxicity, using these cell lines.

Higher levels of HMGCR were observed, at both the transcript and protein level, in Huh7 cells compared to RD muscle cells. Although cholesterol synthesis occurs in almost all cells, the liver is the main site of cholesterol biosynthesis (Catapano 2007; Shitara and Sugiyama 2006). Therefore, the presence of a higher level of HMGCR in Huh7 liver cells is not entirely unexpected, although such a large difference, particularly in the protein levels, was surprising. It is perhaps interesting to note that there is a larger fold-difference in HMGCR expression levels between liver and muscle cells than observed in cholesterol levels. This difference might reflect altered rates of efflux of cholesterol from these cells, or that liver cells have a larger reserve of metabolic capacity for the synthesis of cholesterol compared to muscle cells. 
A difference was also found between HMGCR expression in RD-U and RD-D cells, with much lower levels of expression in RD-D cells. One potential explanation could be a greater need of RD-U cells to produce cholesterol for the synthesis of new membranes, which is reduced as RD cells differentiate. It has also been suggested that HMGCR could play an important role in the induction of differentiation, in particular due to the production of isoprenoids for protein prenylation. In rat L6 skeletal muscle cells HMGCR was found to be upregulated at a very early stage of differentiation ( 6 hours), followed by a subsequent downregulation to allow differentiation to occur (Martini et al. 2009). Since RD-D cells have been allowed to differentiate for 5 days prior to experiments (section 2.2.1.9), it is possible that this downregulation is what is being observed here.

It would be interesting to know whether these effects are observed only in this rate-limiting enzyme, or whether this is also the case for other enzymes in the cholesterol synthesis pathways and for the total flux through the biosynthetic pathway in these cell lines. The different levels of HMGCR expression between the cell types may need to be taken into account when interpreting results from future experiments.

\subsubsection{The Effect of Statin Treatment on Total Cellular Cholesterol}

Having shown the presence of HMGCR in all cell types, subsequent data demonstrated that cholesterol was also present in all cell types of interest and decreases in total cellular cholesterol on statin treatment were observed.

In this study, a period of 48 hours was used for statin treatment prior to cholesterol quantification. This remained consistent with the conditions used for MTT assays as well as taking studies from the literature into account. Previously one research group reported seeing no effect on total cellular cholesterol after $\mathrm{C} 2 \mathrm{C} 12$ mouse myoblasts and HepG2 human liver cells were treated with $10 \mu \mathrm{M}$ simvastatin for 6 or 18 hours (Mullen et al. 2010). However, another research group was able to observe a decrease in intracellular cholesterol in HepG2 cells after 24 and 48 hour treatment with a $1 \mu \mathrm{M}$ concentration of a range of statins (Kobayashi et al. 2008). Given that 
cholesterol synthesized within a cell will have many fates, including sub-cellular sequestration, it is possible that cells are buffered against acute exposure to statins due to sub-cellular pharmacokinetic considerations. Chronic exposure may have a more unequivocal effect, but this must be balanced with the increased cell toxicity associated with such prolonged exposures.

Three statins were chosen for use in this cholesterol analysis: simvastatin (acid), cerivastatin and pravastatin. Simvastatin remains one of the most frequently prescribed statins in the UK (Bakhai et al. 2012; Jick et al. 2012) and, together with cerivastatin, showed the greatest difference in toxicity between RD muscle and Huh7 liver cells (Figure 4-2). The open acid form of simvastatin was used here since this is the form that is biologically active, without need for prior metabolism (Shitara and Sugiyama 2006). Cerivastatin is also of interest in this study due to its high incidence of myopathy in patients (Bolego et al. 2002). In contrast, pravastatin has shown much lower levels of toxicity both in MTT assays (Figure 4-2) and in the clinic (Staffa et al. 2002), as well as having shown less of a difference in toxicity between RD muscle and Huh7 cells compared to simvastatin or cerivastatin (Figure 4-2). It can therefore be used for comparison.

There appeared to be decreases in cholesterol content of Huh7 and RD-D cells in response to $1 \mu \mathrm{M}$ cerivastatin treatment, and in Huh7 cells in response to $10 \mu \mathrm{M}$ simvastatin and pravastatin treatment. There were statistically significant decreases in cholesterol in RD-D cells with $10 \mu \mathrm{M}$ simvastatin $(P=0.0027)$ and pravastatin $(P=0.0023)$.

There was a large degree of variability in the data obtained, which could explain why some of the observed decreases in cholesterol were not found to be statistically significant. This variability was both between replicates within an individual experiment and between experiments. This was particularly evident in the variable levels of cholesterol in control cells. Due to this variability, statistical analysis was not carried out to examine differences in levels of cholesterol and cholesterol reduction on statin treatment between cell lines. However, it appeared that there may be slightly lower levels of cholesterol in RD 
muscle compared to Huh7 liver cells, which could be explained by the lower level of HMGCR in these cells. There also seemed to be no obvious differences in cholesterol reduction in response to statin treatment that could account for the differences in toxicity between the liver and muscle cell lines.

In order to investigate the cause of the variability, experiments were carried out to examine the effect of several variables on the level of cholesterol measured. Since statin stock solutions were made using different solvents, depending on the solubility of the statin, an experiment was carried out to determine whether this could account for the variability in control cholesterol levels between statin treatments. Simvastatin was dissolved in DMSO and, since high levels of DMSO can be toxic to cells, stock was always diluted by 1000 -fold to ensure that the final percentage of DMSO in cell medium did not exceed $0.1 \%$. Cerivastatin and pravastatin were both dissolved in water. No significant difference was found in cholesterol levels between Huh7 cells in either 6-well plates or T25 flasks treated with $0.1 \%$ DMSO, water or $1 \%$ DMSO.

Another potential cause of discrepancy between experiments is cell density. Although cells are seeded at the same initial density, a variety of factors, such as cell passage number, can lead to slight differences in cell growth rate and, ultimately, in cell density. However, no difference was observed in normalised cholesterol levels between Huh7 cells seeded in T25 flasks at 3 different seeding densities.

These two experiments using untreated Huh7 cells were therefore unable to determine the cause of the variation in the data. Nevertheless, despite this variation, the experiments gave us sufficient information to progress as they clearly showed that there was a cholesterol pool in both of our model cell lines, Huh7 and RD, and that statin treatment did result in a decrease in total cellular cholesterol level. 


\subsubsection{Choice of Statin Concentration}

With the use of xenobiotics in in vitro models there is always the issue of choosing therapeutically relevant concentrations (Bjorkhem-Bergman et al. 2011). With simvastatin, concentrations of up to $1 \mu \mathrm{M}$ have been observed in the plasma of patients (Mullen et al. 2010), meaning that the concentrations used for the cholesterol analysis here are not too dissimilar from the physiological range.

However it has also been suggested that localised concentrations in some tissues could be higher than this, due to the activity of transporters, and that this could be variable between individuals (Sirvent et al. 2005). Indeed in treated rats simvastatin concentration was found to be 4-fold higher in the liver than in the plasma, with an even greater difference for cerivastatin (Sidaway et al. 2009). Statin concentrations have been found to be a third lower in rat skeletal muscle than in plasma for simvastatin, with an even greater difference with cerivastatin (a sixth lower) and rosuvastatin (1/16) (Sidaway et al. 2009). Similar findings have been reported in guinea pig skeletal muscle for cerivastatin, rosuvastatin and atorvastatin (Madsen et al. 2008). The plasma-tomuscle ratios are not currently known for humans (Knauer et al. 2010) and localised concentrations in muscle may be increased in patients when statin treatment is given in combination with a fibrate or a CYP inhibitor (Mullen et al. 2010).

The biological effect of statins is a decrease in cholesterol (Chong et al. 2001) and it has been shown here that decreases in cholesterol can be observed on treatment with $10 \mu \mathrm{M}$ simvastatin and pravastatin, and $1 \mu \mathrm{M}$ cerivastatin (section 4.3.2). Therefore, since these are the statin concentrations which reproduce the expected biological effect of the statins, and are concentrations at which RD muscle cell toxicity can be observed with simvastatin and cerivastatin, these are the concentrations that were used in future experiments. 


\subsection{Summary}

Responses of Huh7 liver and RD muscle cells to statin treatment have been characterised in terms of both cellular viability and cholesterol metabolism.

Using MTT assays, significant reductions in cell viability were observed for all three cell types (Huh7, RD-U and RD-D) on treatment with simvastatin (acid and lactone), lovastatin (acid and lactone), cerivastatin, fluvastatin, atorvastatin, rosuvastatin and pravastatin in a dose-dependent manner. The largest reductions in cell viability were observed with the more lipophilic statins: simvastatin (acid), lovastatin (acid) and cerivastatin, whereas loss of cell viability was only observed with very high concentrations of the hydrophilic pravastatin ( $1 \mathrm{mM}$ and greater). Higher cell toxicity was also observed for all statins in the RD cells, either differentiated or undifferentiated, compared to the Huh7 cells, where this difference was statistically significant with simvastatin, cerivastatin, atorvastatin and rosuvastatin. Taken together, these data represent the first comprehensive comparative analysis of statin toxicity in human liver and muscle cell lines.

The level of HMGCR, the rate-limiting enzyme in cholesterol biosynthesis and the enzyme inhibited by the statins, was analysed. HMGCR was found to be expressed at both the transcript and protein level in all cell types, although to a lesser extent in RD muscle cells (particularly when differentiated). Decreases in total cellular cholesterol were observed on treatment with $10 \mu \mathrm{M}$ simvastatin and pravastatin, and $1 \mu \mathrm{M}$ of cerivastatin. Since these are the concentrations that replicate the biological effect of the statins (a decrease in cholesterol), these are the concentrations that were then used in future experiments, examining other molecular changes that occur in these cells on statin treatment. 


\section{The Effects of Statins on Prenylation in Liver and Muscle Cells}

\subsection{Introduction}

Due to the large number of intermediates in the mevalonate pathway (Figure 1-3), inhibition of 3-hydroxy-3-methylglutaryl coenzyme A reductase (HMGCR) by statins not only inhibits cholesterol synthesis but also impacts upon a number of other cellular processes, including protein prenylation (Vaklavas et al. 2009).

Farnesylpyrophosphate (FPP) and geranylgeranylpyrophosphate (GGPP) are intermediates of the mevalonate pathway. In protein prenylation, these isoprenoids donate farnesyl (15-carbon) or geranylgeranyl (20-carbon) groups, respectively, for post-translational covalent addition to cysteine residues at or near the C-terminus of proteins. This addition is thought to be essential for the biological function of many proteins by enabling them to anchor to cell membranes, specifying cellular localisation (McTaggart 2006). There is increasing evidence that disruption of various prenylation targets could lead to myopathy (Blanco-Colio et al. 2002; Matzno et al. 2005; Sakamoto et al. 2011).

The three distinct protein prenyltransferase enzymes that are responsible for the addition of prenyl groups to proteins are farnesyltransferase (FT), geranylgeranyltransferase-I (GGT-I) and geranylgeranyltransferase-II (GGT-II). Other than a small number of specific cases of overlap (sections 1.3.5.2i and ii), each enzyme is thought to have a distinct subset of substrates (Figure 5-1). Substrates for FT include Ras guanine nucleotide-binding proteins (GTPases) and the nuclear lamins; GGT-I adds geranylgeranyl groups to Ras-related GTPases, including of the Ras and Rho subfamilies, and the $\gamma$ subunits of heterotrimeric G-proteins; GGT-II is responsible for the prenylation of Rasrelated GTPases in the Rab subfamily (Casey and Seabra 1996). 


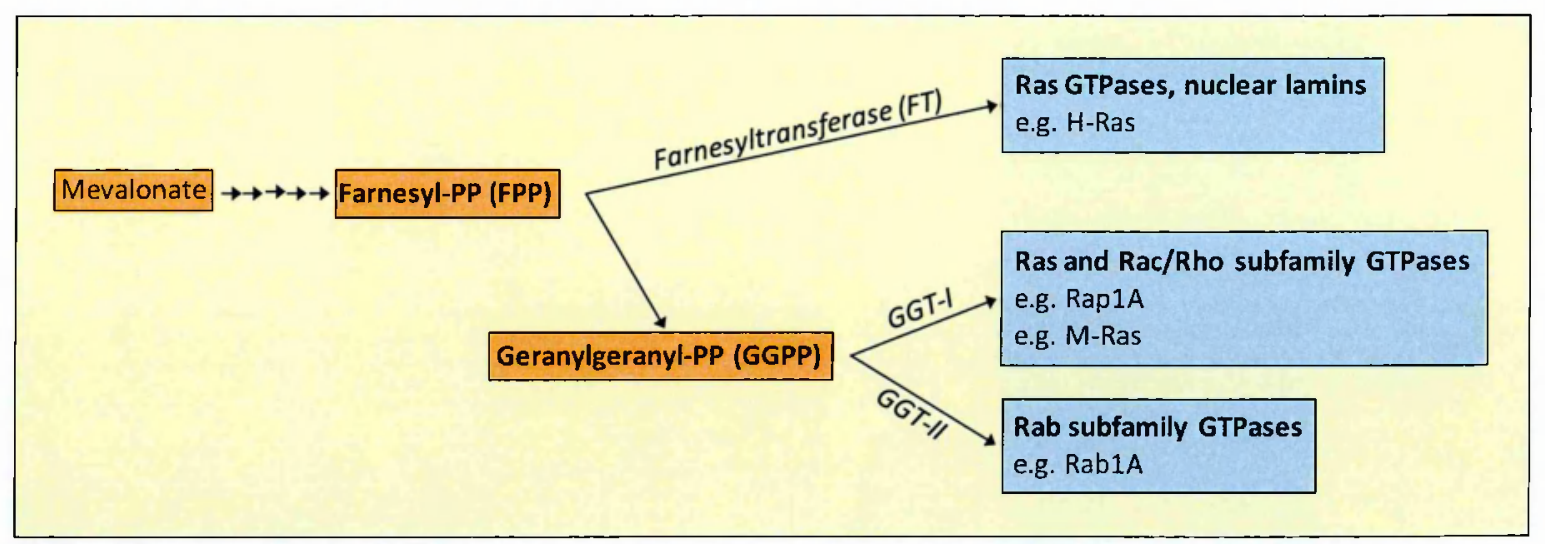

\section{Figure 5-1 Protein prenylation}

Farnesylpyrophosphate (FPP) and geranylgeranylpyrophosphate (GPP), isoprenoid products of the mevalonate pathway, donate farnesyl or geranylgeranyl groups respectively for the posttranslational prenylation of proteins. The 3 prenyltransferase enzymes which catalyse this step are farnesyltransferase (FT), geranylgeranyltransferase-I (GGT-I) and geranylgeranyltransferase-II (GGT-II). Each enzyme has a distinct group of substrates.

Work discussed in previous chapters has established human liver and muscle cell-based models and characterised their response to statin treatment, in terms of both cell viability and changes in cholesterol levels. In this chapter, the effect of statin treatment on the cellular process of protein prenylation, by each of the three prenyltransferase enzymes, was examined in these liver and muscle models, with the hypothesis that statins would preferentially disrupt prenylation within muscle, the site of observed human toxicity.

\subsection{Rationale for Experimental Design}

Protein was extracted from Huh7, RD-U and RD-D cells treated with vehicle control, statin or mevalonic acid in addition to statin, and then subjected to analysis by western blot (section 2.2.3) to detect any changes in the prenylation status of selected target proteins as well as to examine the expression of the prenyltransferase enzymes.

Simvastatin (acid), cerivastatin and pravastatin, were used in this analysis as these are the statins used previously to examine the effects of statin treatment on total cellular cholesterol (section 4.3.2). A decrease in cholesterol, the expected biological effect of the statins, was observed on treatment with $10 \mu \mathrm{M}$ simvastatin and pravastatin, and $1 \mu \mathrm{M}$ cerivastatin, and so these were the initial 
concentrations also used here. Both simvastatin and cerivastatin treatments were previously shown to result in greater toxicity in RD muscle cells than Huh7 liver cells (section 4.2). In contrast, pravastatin treatment resulted in toxicity only at high concentrations and showed no statistically significant difference in toxicity between cell lines, therefore it can be used for comparison.

Mevalonic acid (mevalonate) is an intermediate in the mevalonate pathway that is just downstream of the site of statin inhibition. Its addition has been shown to compensate for the statin-induced block of the mevalonate pathway, thereby indicating whether any effects that are observed are due to the specific inhibition of HMGCR or to non-specific effects.

Cells were also treated with 3 inhibitors of prenylation: FTI-277, GGTI-2133 and perillyl alcohol. FTI-277 is a trifluoroacetate salt that inhibits FT by acting as a highly potent Ras CAAX peptidomimetic, mimicking the Ras C-terminal CAAX FT recognition motif and site of prenylation (Lerner et al. 1995; Sigma-Aldrich 2011a). GGTI-2133 is a non-thiol peptidomimetic that inhibits GGT-I with an 140-fold selectivity over FT (Sigma-Aldrich 2011b). There are only a limited number of specific inhibitors of GGT-II available (Leung et al. 2006), none of which were commercially obtainable. Therefore perillyl alcohol was used, which is a monoterpene that inhibits both GGT-I and II, with 2-fold greater potency for the inhibition of GGT-II (Ren et al. 1997; Tanaka et al. 2010). 


\subsection{The Effects of Statins on Prenylation}

The small GTPases represent the largest group of proteins where prenylation is vital for their membrane localisation and function (McTaggart 2006). In this analysis, the prenylation of several small GTPases in response to statin treatment was examined. Rap1A was chosen as a representative small GTPase prenylated by GGT-I, with H-Ras as a substrate of FT and with Rab1A as a substrate of GGT-II.

\subsubsection{Rap1A}

Rap1A is a 22kDa Ras-related GTPase which is prenylated by GGT-I and is thought to play roles in cell adhesion, the establishment of cell polarity and the regulation of cell-cell contacts (Frische and Zwartkruis 2010). It is one of two very closely related isoforms of Rap1 (Bos 1997; Frische and Zwartkruis 2010) and it has previously been shown that levels of unprenylated Rap1A increase on treatment with simvastatin in both HepG2 liver cells and $\mathrm{C} 2 \mathrm{C} 12$ mouse myotubes (Mullen et al. 2010). It was important to verify whether this effect is also observed in human cell lines and whether there is a difference between the effects of different statins.

In order to investigate the geranylgeranylation state of Rap1A two antibodies were used. One antibody (referred to as Rap1A) recognises only the unprenylated form of the protein (Antoine et al. 2010; Reszka et al. 2001) and the other (referred to as Rap1) recognises both Rap1 isoforms whether prenylated or unprenylated (Reszka et al. 2001), thereby giving an indication of total Rap1 protein expression. This meant that it was possible to use total protein extracts in this case (section 2.2.3.1).

There was no unprenylated Rap1A detected in any of the control samples, suggesting that this prenylation step is highly efficient. Unprenylated Rap1A was observed in all three cell types following treatment with $10 \mu \mathrm{M}$ simvastatin or $1 \mu \mathrm{M}$ cerivastatin, but not $10 \mu \mathrm{M}$ pravastatin (Figure 5-2). In all cases, this was largely rescued on co-addition of mevalonic acid, showing that the increase in unprenylated Rap1A was due to statin inhibition of HMGCR rather than a non-specific effect. 
A] Huh7

B]

RD-U
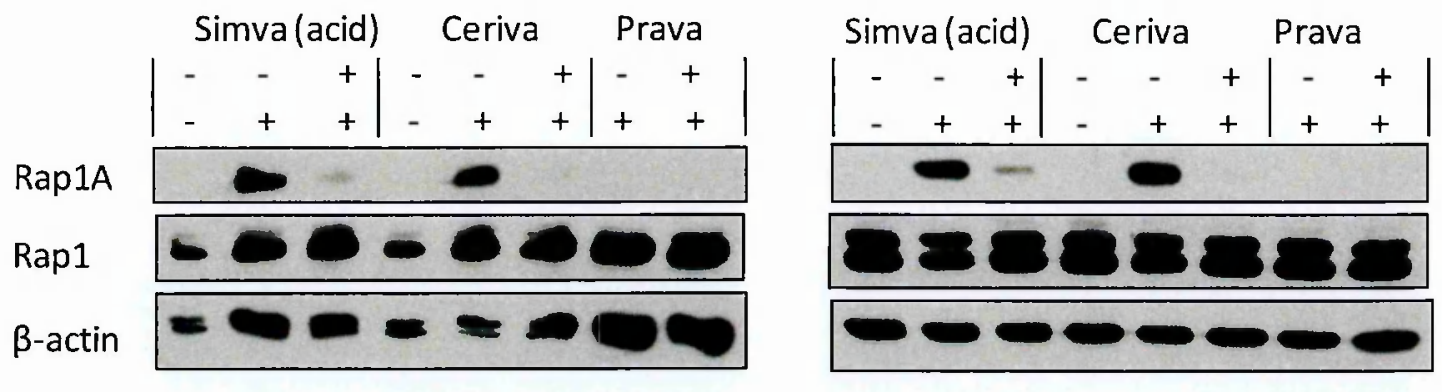

mevalonic acid statin

C] RD-D

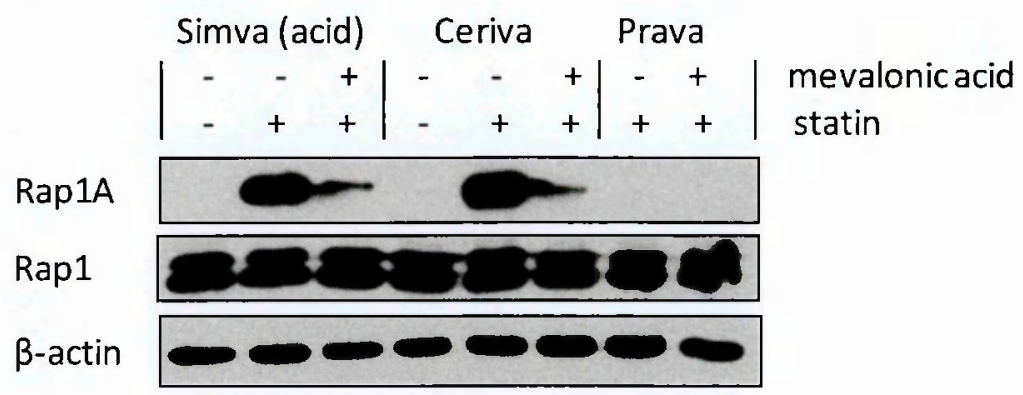

Figure 5-2 Effect of statin treatment on Rap1A prenylation

Total protein was extracted from cells treated for 48 hours with vehicle control, statin, or mevalonic acid in addition to statin. Simvastatin (simva) and pravastatin (prava) were used at a concentration of $10 \mu \mathrm{M}$ and cerivastatin (ceriva) at $1 \mu \mathrm{M}$. DMSO was used as a vehicle control for simvastatin and water for cerivastatin and pravastatin. Mevalonic acid was used in excess, at $100 \mu \mathrm{M}$. Protein concentration was determined using the Lowry method and $30 \mu \mathrm{g}$ was separated by SDS-PAGE and transferred to PVDF membrane. The level of unprenylated Rap1A (21 kDa) was detected in Huh7 (A), undifferentiated RD (RD-U) (B) and differentiated RD (RD-D) (C) cells using a specific antibody, and compared to the levels of total Rap1 and of the loading control, $\beta$-actin. Protein size was determined by comparison to a coloured protein marker. Western blots are representative of three independent experiments.

$\beta$-actin was used as a loading control, as its expression is unchanged on statin treatment and therefore any variation detected can be attributed to uneven protein loading. The only changes observed in total Rap1, in samples from Huh7 cells (Figure 5-2A), corresponded to similar differences in $\beta$-actin. Therefore, the observed increase in the unprenylated form of Rap1A was not due to an increase in protein expression upon statin treatment. There were also no changes in $\beta$-actin that would allow uneven loading to account for the changes observed in unprenylated Rap1A. 
In order to investigate whether this detection of unprenylated Rap1A was due to a specific effect of the statins on the process of prenylation, cells were treated with the prenyltransferase inhibitors described in section 5.2. Treatment with the GGT-I inhibitor GGTI-2133 resulted in observation of unprenylated Rap1A in all cell types (Figure 5-3).

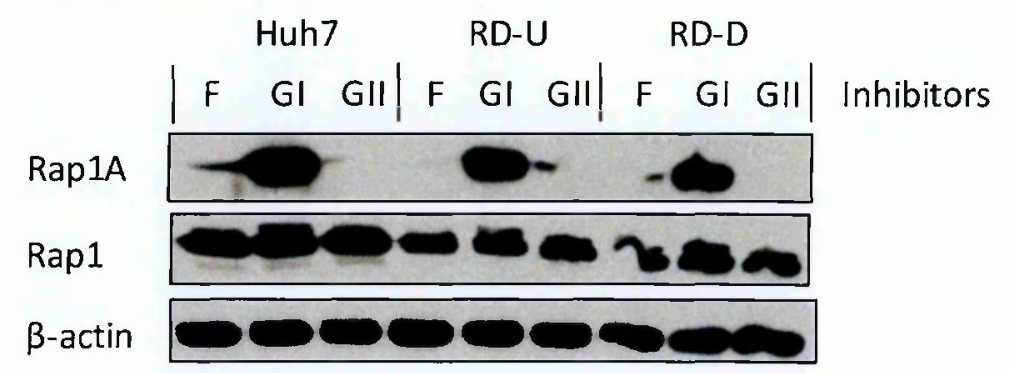

Figure 5-3 Effect of prenylation inhibitors on Rap1A prenylation

Total protein was extracted from Huh7, RD-U and RD-D cells treated for 48 hours with $10 \mu \mathrm{M}$ farnesyltransferase inhibitor FTI-277 (F), 10 $1 \mathrm{M}$ geranylgeranyltransferase-l inhibitor GGTI-2133 (GI) or $100 \mu \mathrm{M}$ perillyl alcohol (GII), and $30 \mu \mathrm{g}$ was separated by SDS-PAGE. Western blots are representative of three independent experiments.

This was similar to the effect seen with simvastatin and cerivastatin treatment. However, treatment with neither the FT inhibitor FTI-277 nor perillyl alcohol resulted in any comparable detection of unprenylated Rap1A. Since specifically inhibiting GGT-I has the same effect as statin treatment, this supports the idea that statins are having an inhibitory effect on protein prenylation and confirms that Rap1A is a GGT-I substrate.

\subsubsection{Dose-Dependent Effects of Statin Treatment on Rap1A Prenvlation}

Since treatment with $10 \mu \mathrm{M}$ simvastatin and $1 \mu \mathrm{M}$ cerivastatin treatment resulted in a similar increase in unprenylated Rap1A in all cell types, the dosedependent effects of simvastatin and cerivastatin on Rap1A prenylation were examined. For this, concentrations were chosen from across the range of the dose-response curves (Figure 4-2). The greatest effects on treatment with $30 \mu \mathrm{M}$ of these statins, the highest concentration used in this analysis, previously led to the reduction of cell viability to $60 \%$ in Huh7 cells, $23 \%$ in RD$\mathrm{U}$ cells and $39 \%$ in RD-D cells; where $100 \%$ was defined as cell viability on treatment with vehicle control. 
On simvastatin treatment, there was a dose-dependent increase in the inhibition of Rap1A prenylation in all cell types (Figure 5-4), with unprenylated Rap1A observed at all concentrations equal to or greater than $10 \mu \mathrm{M}$ simvastatin in Huh7 cells (Figure 5-4A); in both RD-U and RD-D cells unprenylated Rap1A was evident for all concentrations equal to or greater than $1 \mu \mathrm{M}$ (Figure 5-4B and $\mathrm{C}$ ). Hence, in this model, disruption of Rap1A prenylation occurs at a 10fold lower concentration in muscle than in liver following simvastatin treatment.

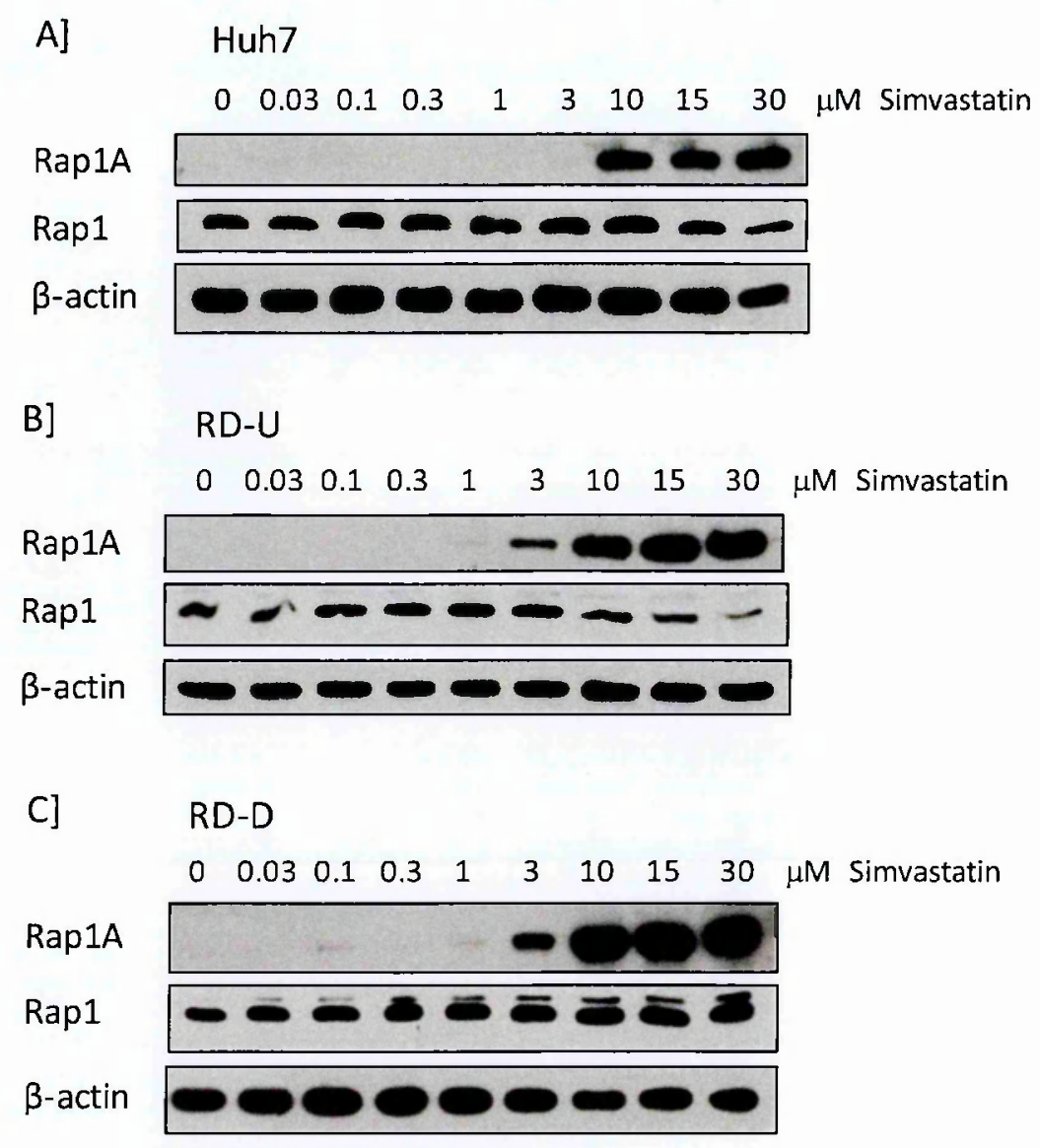

Figure 5-4 Effect of simvastatin treatment on Rap1A prenylation

Total protein was extracted from cells treated for 48 hours with vehicle control (DMSO) or a range of simvastatin concentrations and $15 \mu \mathrm{g}$ was separated by SDS-PAGE. The level of unprenylated Rap1A (21kDa) was detected in Huh7 (A), RD-U (B) and RD-D (C) cells using a specific antibody, and compared to the levels of total Rap1 and of the loading control, $\beta$-actin. Protein size was determined by comparison to a coloured protein marker. 
On cerivastatin treatment, there was likewise a dose-dependent decrease in Rap1A prenylation (Figure 5-5). Prenylation of Rap1A was also inhibited at 10fold lower concentrations of cerivastatin than simvastatin, with some unprenylated Rap1A being observed even with concentrations of cerivastatin as low as $0.1 \mu \mathrm{M}$ in all cell types. Cerivastatin seemed to also have a greater effect on the disruption of prenylation in muscle compared to liver, although the difference was only 3-fold compared to the previously observed 10 -fold difference in sensitivity to simvastatin.

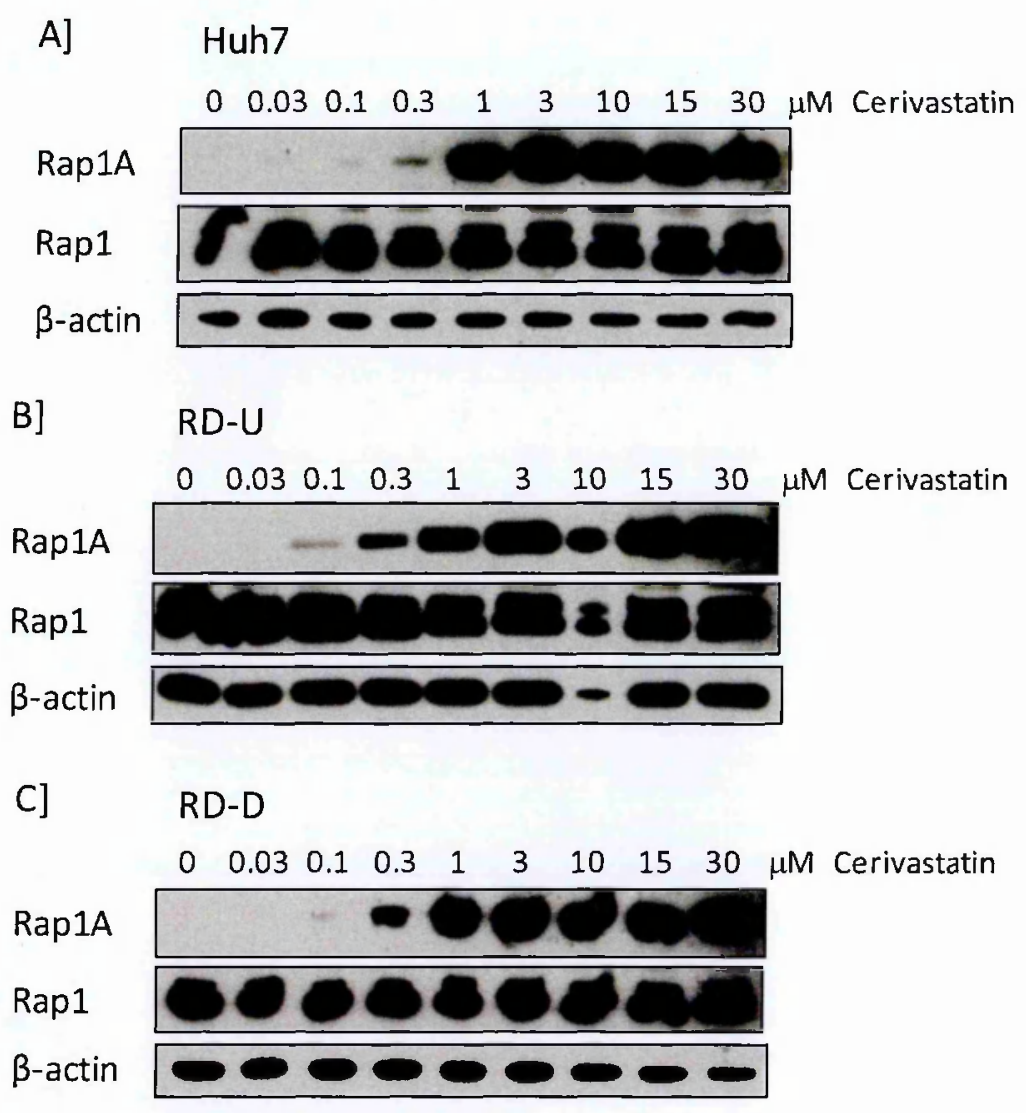

Figure 5-5: Effect of cerivastatin treatment on Rap1A prenylation

Total protein was extracted from cells treated for 48 hours with vehicle control (DMSO) or a range of cerivastatin concentrations and $15 \mu \mathrm{g}$ was separated by SDS-PAGE. The level of unprenylated Rap1A (21kDa) was detected in Huh7 (A), RD-U (B) and RD-D (C) cells using a specific antibody, and compared to the levels of total Rap1 and of the loading control, $\beta$-actin.

Overall, both simvastatin and cerivastatin showed a dose-dependent inhibition of Rap1A prenylation. However, cerivastatin showed effects at lower concentrations than simvastatin, and prenylation was inhibited a lower concentrations in muscle compared to liver, in this model. The observation of 
greater sensitivity to disruption of prenylation in muscle compared to liver further supports a mechanism where disruption of intermediates in the mevalonate pathway is indeed a cause of statin-induced muscle toxicity.

\subsubsection{H-Ras}

In order to examine whether statin treatment had similar effects on the prenylation of other small GTPases, further western blot analysis was undertaken. These subsequent analyses were carried out using membrane and cytosolic protein extracts (section 2.2.3.2), rather than total protein extracts as, unlike the Rap1A antibody used, the other antibodies did not directly distinguish between prenylated and unprenylated forms of the protein. Since, under normal conditions, GTPases are anchored to cell membranes by their added prenyl groups (Jin et al. 1996), a reduction in prenylation will result in less protein able to remain in the membrane and will lead to possible detection in cytosolic fractions. Therefore, observing the change in subcellular localisation of the protein may be used as an indirect measure of the inhibition of prenylation by statins (Blanco-Colio et al. 2002; Casey et al. 1989; Coxon et al. 2005).

$\mathrm{H}$-Ras is one of four classical Ras proteins which, along with N-Ras, K-Ras4A and K-Ras4B, was identified originally as an oncogene (Guo et al. 2005). HRas acts as a molecular switch; The binding of GTP induces a conformational change in the protein, allowing it to then bind to downstream effectors (Castro et al. 2005) and initiate protein kinase cascades, such as the MAPK (mitogenactivated protein kinase) cascade (Matozaki et al. 2000; Vojtek and Der 1998), in order to exert its effects on gene transcription, cellular growth and differentiation (Keduka et al. 2009). The addition of a farnesyl group to H-Ras by FT is essential for its membrane anchoring, and is thereby also essential for allowing it to carry out its role in cellular signalling (Dirks and Jones 2006). 
In agreement with this, H-Ras was found almost exclusively in the membrane fraction of control cells (Figure 5-6). Cerivastatin treatment resulted in a reduction in the amount of $\mathrm{H}$-Ras in the membrane fraction, indicating decreased prenylation. This reduction was seen in all cell types, with H-Ras being detected in the corresponding cytosolic fractions in Huh7 and RD-D cells. This effect of cerivastatin on H-Ras membrane association was largely rescued by mevalonic acid co-treatment, demonstrating this to be a specific impact on the mevalonate pathway.

A]

Huh7

B] RD-U
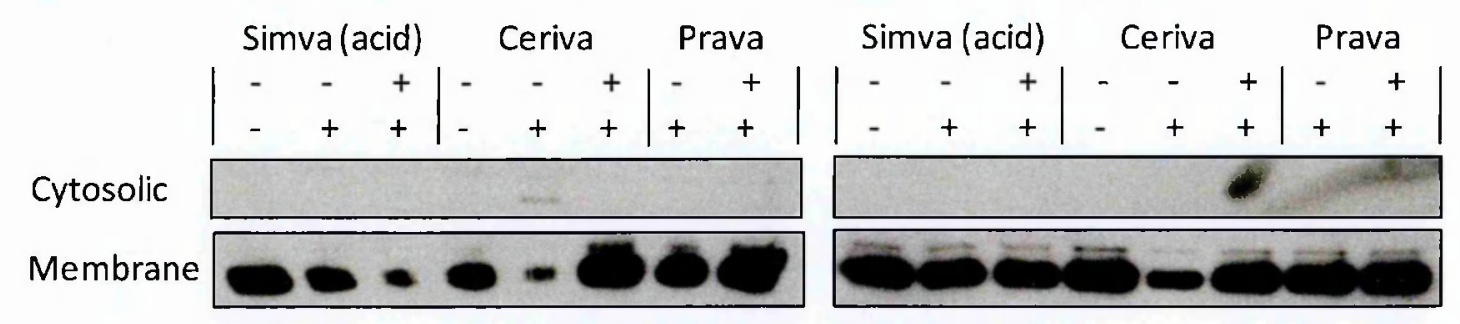

C] RD-D

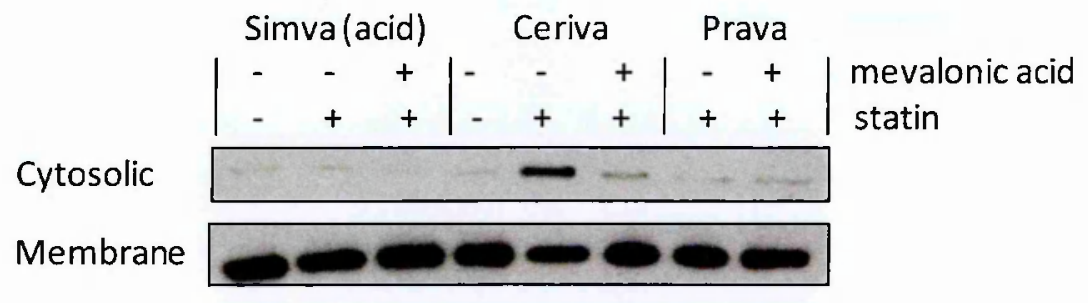

Figure 5-6 Effect of statin treatment on membrane association of H-Ras

Fractions enriched with either cytosolic or membrane protein were extracted from cells treated for 48 hours with vehicle control, statin, or mevalonic acid in addition to statin, and $4 \mu \mathrm{g}$ was separated by SDS-PAGE. Simvastatin and pravastatin were used at a concentration of $10 \mu \mathrm{M}$ and cerivastatin at $1 \mu \mathrm{M}$. DMSO was used as a vehicle control for simvastatin and water for cerivastatin (and pravastatin). Mevalonic acid was used in excess, at $100 \mu \mathrm{M}$. The level of $\mathrm{H}$ Ras (21kDa) in each fraction was detected in Huh7 (A), RD-U (B) and RD-D (C) cells using a specific antibody. Protein size was determined by comparison to a coloured protein marker.

In contrast, treatment with pravastatin had no effect on the subcellular localisation of H-Ras in any of the cell types, and the only apparent reduction of membrane-associated $\mathrm{H}$-Ras due to simvastatin treatment was in Huh7 cells co-treated with mevalonic acid. The latter was surprising, since previously the addition of mevalonic acid rescued the effect of statins; it is possible that this 
could be an artefact arising from a problem with this section of the original gel or membrane, which may be clarified by further analysis of this membrane.

Since treatment with cerivastatin resulted in a change in $\mathrm{H}$-Ras localisation in all cells, cells were treated with prenyltransferase inhibitors to see if this would lead to similar changes. As expected, there was a reduction in $\mathrm{H}$-Ras observed in the membrane fraction of RD-U and RD-D cells treated with the FT inhibitor FTI277 (Figure 5-7), providing evidence that the changes in H-Ras localisation observed in muscle cells on statin treatment are due to inhibition of farnesylation. Interestingly, in Huh7 liver cells a reduction in membrane associated $\mathrm{H}$-Ras was observed on treatment with geranylgeranyltransferase inhibitor GGTI-2133 and not with FTI-277, as in RD cells.

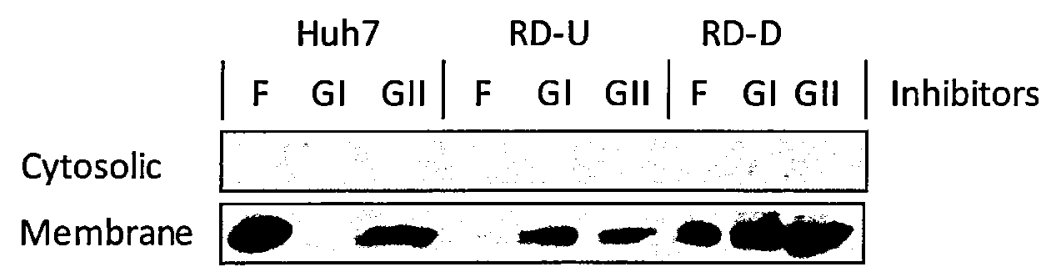

Figure 5-7 Effect of prenylation inhibitors on membrane association of H-Ras

Fractions enriched with either cytosolic or membrane protein were extracted from cells treated for 48 hours with $10 \mu \mathrm{M}$ farnesyltransferase inhibitor FTI-277 (F), $10 \mu \mathrm{M}$ geranylgeranyltransferase-I inhibitor GGTI-2133 (GI) or $100 \mu \mathrm{M}$ perillyl alcohol (GII) and $4 \mu \mathrm{g}$ was separated by SDS-PAGE. The level of H-Ras $(21 \mathrm{kDa})$ in each fraction was detected using a specific antibody.

In general, inhibition of prenylation resulted in a decrease of membraneassociated $\mathrm{H}$-Ras, an effect also observed in all cell types upon cerivastatin treatment. Therefore, it can be inferred that cerivastatin has an effect on the prenylation of the small GTPase H-Ras in addition to that of Rap1A. 


\subsubsection{M-Ras}

M-Ras (also known as R-Ras3 due to its similarities to R-Ras and R-Ras2 (Ehrhardt et al. 1999)) is one of the more recently described members of the Ras subfamily of GTPases, having been originally cloned from the CDNA libraries of mouse skeletal muscle and rat brain. It was initially thought that the expression of this $29 \mathrm{kDa}$ protein was restricted to brain, muscle and heart (Kimmelman et al. 1997; Matsumoto et al. 1997). However, it has since been shown that its expression is actually much more widespread, including spleen, thymus, liver, lung, pancreas, placenta, skin, kidney, fibroblasts, hematopoietic and epithelial cells (Ehrhardt et al. 1999) as well as MCF-7 breast, HeLa cervical and LNCaP prostate cancer cell lines (Castro et al. 2011). M-Ras shows $47 \%$ identity with $\mathrm{H}$-Ras (Ehrhardt et al. 1999) and they share many upstream regulators and downstream effectors. Nevertheless, M-Ras has several distinctive effectors, for example RA-GEF-2 and MR-GEF (Guo et al. 2005), and M-Ras is thought to have evolved independently of H-Ras and other members of the Ras family (Keduka et al. 2009). It is thought that M-Ras, unlike H-Ras, is a substrate of GGT-I (Kimmelman et al: 1997; Matsumoto et al. 1997).

The western blot analysis of M-Ras is not as clear as those western blots looking at $\mathrm{H}$-Ras expression, due to the quality of the available antibody. However, it is still evident that M-Ras is found in the prenylated, membranebound form under normal conditions (Figure 5-8). It is also clear that there is a decrease in the amount of M-Ras in the membrane of RD-U cells (Figure 5-8B), along with a concomitant increase in M-Ras in the cytosol of RD-D (Figure $5-8 C$ ) on cerivastatin treatment, as is also the case with H-Ras (Figure 5-6). These effects were rescued on co-treatment with mevalonic acid. It is unclear as to whether this occurs in Huh7 cells; however a repeat experiment, perhaps with an alternative antibody, would clarify this. 
Cytosolic

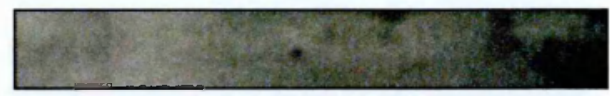

Membrane
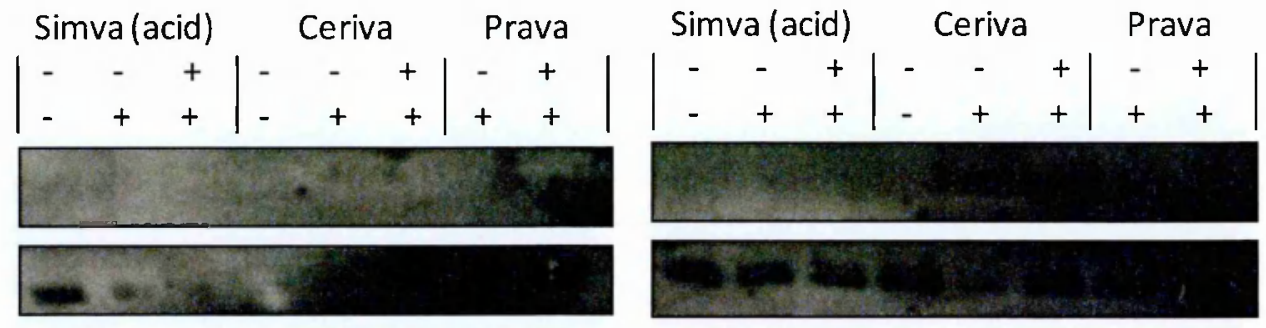

C]

\section{RD-D}

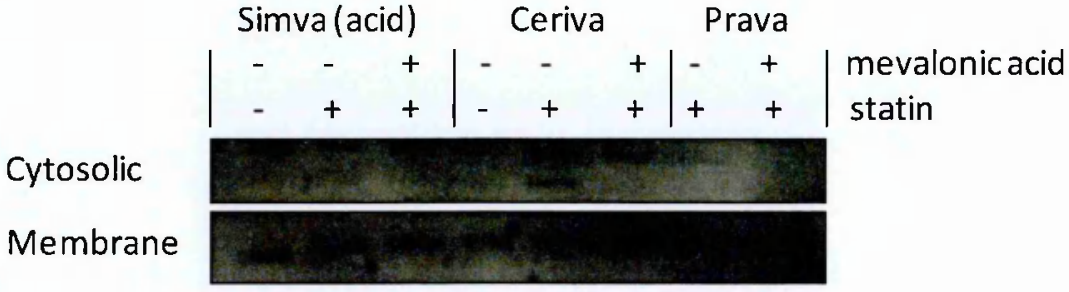

\section{Figure 5-8 Effect of statin treatment on membrane association of M-Ras}

Fractions enriched with either cytosolic or membrane protein were extracted from cells treated for 48 hours with vehicle control, statin, or mevalonic acid in addition to statin, and $4 \mu \mathrm{g}$ was separated by SDS-PAGE. Simvastatin and pravastatin were used at a concentration of $10 \mu \mathrm{M}$ and cerivastatin at $1 \mu \mathrm{M}$. DMSO was used as a vehicle control for simvastatin and water for cerivastatin and pravastatin. Mevalonic acid was used in excess, at $100 \mu \mathrm{M}$. The level of M-Ras (29kDa) in each fraction was detected in Huh7 (A), RD-U (B) and RD-D (C) cells using a specific antibody. Protein size was determined by comparison to a coloured protein marker.

There also appears to be an increase in M-Ras observed in the cytosol of all cells on treatment with the GGT-I inhibitor GGTI-2133 but not with the FT inhibitor FTl-277 (Figure 5-9). This would seem to confirm the predictions that M-Ras is a substrate of GGT-I and again shows statin treatment to have a similar effect to direct inhibition of protein prenylation.

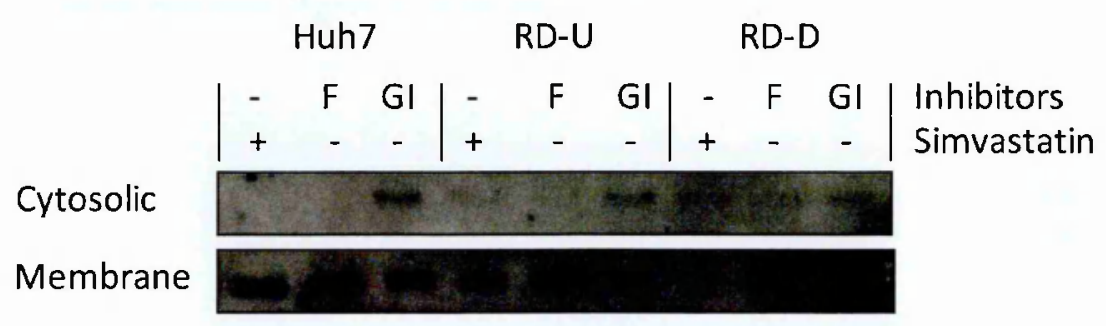

Figure 5-9 Effect of prenylation inhibitors on membrane association of M-Ras

Fractions enriched with either cytosolic or membrane protein were extracted from cells treated for 48 hours with $10 \mu \mathrm{M}$ simvastatin, $10 \mu \mathrm{M}$ farnesyltransferase inhibitor FTI-277 (F) or $10 \mu \mathrm{M}$ geranylgeranyltransferase-I inhibitor GGTI-2133 (GI), and $4 \mu \mathrm{g}$ was separated by SDS-PAGE. The level of M-Ras $(29 \mathrm{kDa})$ in each fraction was detected using a specific antibody. 


\subsubsection{Rab1A}

Rab1 is a 25kDa small GTPase important in the secretory pathway for regulating vesicle traffic from the endoplasmic reticulum (ER) to the Golgiapparatus, as well as intra-Golgi traffic. Rab1 is found in all cell types (Leung et al. 2006), including the liver (Jin et al. 1996) and skeletal muscle (Bao et al. 1998). It has been reported that Rab1 is one of the forms of Rab most susceptible to depletion of GGPP (Tanaka et al. 2010) and recently it has been shown that inactivation of Rab1 may play an important role in fluvastatin toxicity in rat myofibres (Sakamoto et al. 2011; Tanaka et al. 2010). There are two different isoforms of Rab1, Rab1A and Rab1B, which share $92 \%$ identity and are thought to be functionally interchangeable (Nuoffer et al. 1994). Rab1A is the predominant isoform in human skeletal muscle, and so was chosen here as a representative GGT-II substrate.

Western blot analysis showed two distinct bands in the cytosolic fractions of Huh7 and RD-D cells and more faintly in all membrane fractions (Figure 5-10). It could be that these correspond to differently processed forms of the Rab1A protein, with resulting differences in molecular weight, or it could be that the higher molecular weight band represents non-specific binding of the antibody, with Rab1A being represented solely by the lower molecular weight band in these samples. 


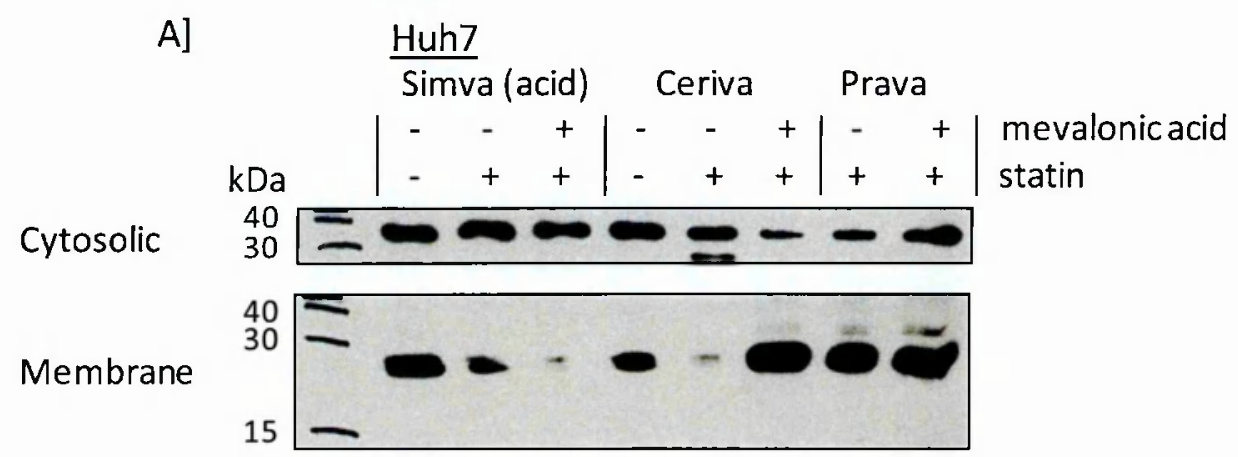

B]

$\underline{\mathrm{RD}-\mathrm{U}}$

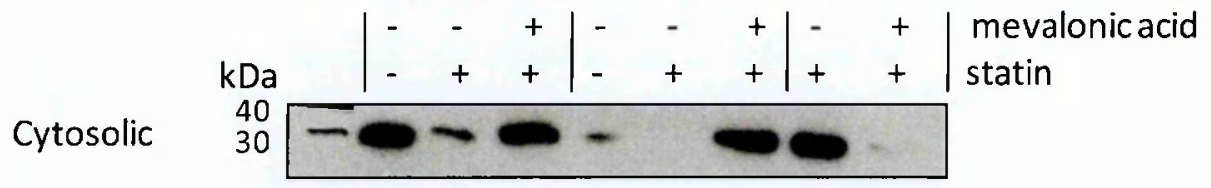

Membrane

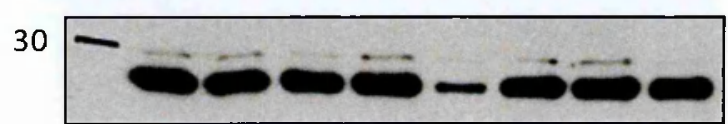

C]

$\underline{\mathrm{RD}-\mathrm{D}}$

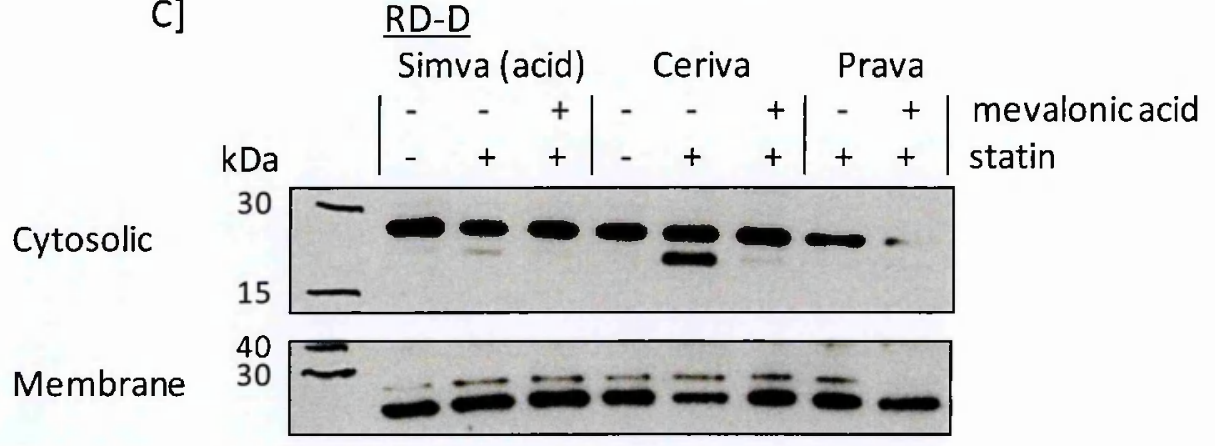

Figure 5-10 Effect of statin treatment on membrane association of Rab1A

Fractions enriched with either cytosolic or membrane protein were extracted from cells treated for 48 hours with vehicle control, statin, or mevalonic acid in addition to statin, and $4 \mu \mathrm{g}$ was separated by SDS-PAGE. Simvastatin and pravastatin were used at a concentration of $10 \mu \mathrm{M}$ and cerivastatin at $1 \mu \mathrm{M}$. DMSO was used as a vehicle control for simvastatin and water for cerivastatin and pravastatin. Mevalonic acid was used in excess, at $100 \mu \mathrm{M}$. The level of Rab1A (25kDa) in each fraction was detected in Huh7 (A), RD-U (B) and RD-D (C) cells using a specific antibody. Approximate location of the relevant section of the coloured protein marker is shown.

Treatment with cerivastatin, but not simvastatin or pravastatin, resulted in a decrease in the amount of the lower molecular weight protein observed in the membrane fractions of all cells (Figure 5-10), which is similar to the effects of the statin on membrane association of H-Ras (Figure 5-6) and M-Ras (Figure 5-8). This lower molecular weight protein was detected in cytosolic fractions only from Huh7 (Figure 5-10A) and RD-D (Figure 5-10C) cells treated with cerivastatin and from RD-D (Figure 5-10C) cells treated with simvastatin. 
There also seems to be less Rab1A in the membrane fraction from Huh7 cells treated with simvastatin and mevalonic acid. This effect is the same as that seen when these same PVDF membranes were analysed for $\mathrm{H}$-Ras expression (Figure 5-6). This is further indication that there may have been problems with the original protein loading or with the processing of this particular area of the PVDF membrane.

Treatment with perillyl alcohol, to inhibit both GGT-I and GGT-II, resulted in a lower amount of Rab1A observed in the membrane fraction from RD-U cells (Figure 5-11). However, it seems that this effect was observed to an even greater extent on inhibition of FT in RD-U and RD-D cells, and on inhibition of GGT-I in Huh7 cells. This pattern of reduced membrane-association seems to be the same as when the same samples were analysed for localisation of $\mathrm{H}$ Ras on treatment with prenyltransferase inhibitors (Figure 5-7), despite the fact that $\mathrm{H}$-Ras and Rab1A are reported to be prenylated by different prenyltransferase enzymes. This is surprising, and could indicate that either the prenyltransferase enzymes, or these prenyltransferase inhibitors, are not as specific under all conditions as traditionally thought.

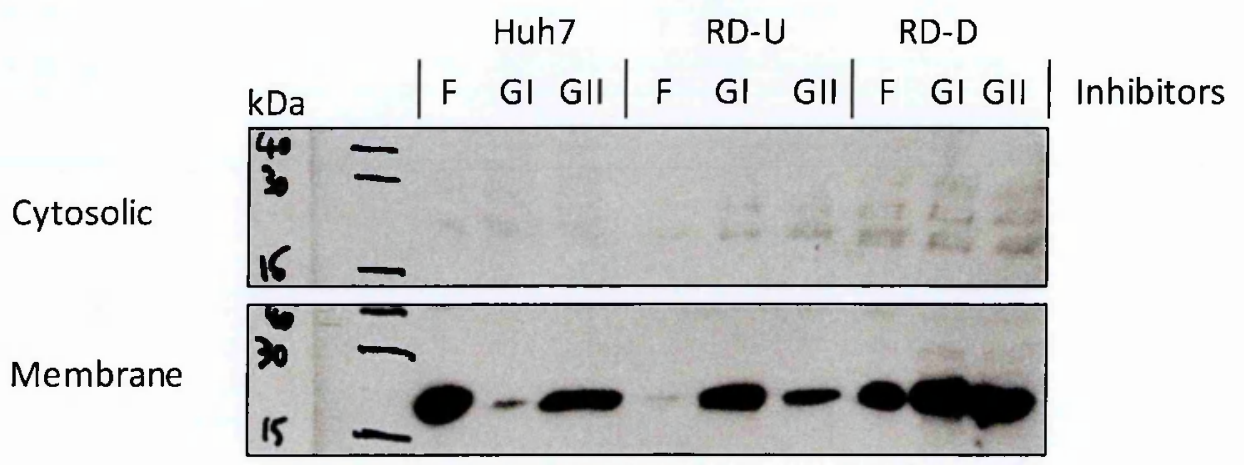

Figure 5-11 Effect of prenylation inhibitors on membrane association of Rab1A

Fractions enriched with either cytosolic or membrane protein were extracted from cells treated for 48 hours with $10 \mu \mathrm{M}$ farnesyltransferase inhibitor FTI-277 $\quad(\mathrm{F}), \quad 10 \mu \mathrm{M}$ geranylgeranyltransferase-I inhibitor GGTI-2133 (GI) or 100 $\mu \mathrm{M}$ perillyl alcohol (GII), and $4 \mu \mathrm{g}$ was separated by SDS-PAGE. The level of Rab1A $(25 \mathrm{kDa})$ in each fraction was detected. Approximate location of the relevant section of the coloured protein marker is shown. 
Another interesting point to note is that neither of the two molecular weight bands can be observed in the cytosolic fractions of cells treated with the prenyltransferase inhibitors, and only the lower molecular weight band can be observed in membrane fractions. The PVDF membranes with these samples were prepared at a later time than the membranes with the samples from statin treated cells. It could therefore be that these later western blot analyses are showing greater Rab1A specificity.

In summary, treatment with both cerivastatin and prenyltransferase inhibitors led to a reduction in membrane-associated Rab1A in both muscle and liver cells. However, this analysis was complicated by the detection of several protein bands, in both the cytosolic and membrane fractions, which could be due to non-specific antibody binding. 


\subsubsection{Lactate Dehydrogenase (LDH)}

In order to assess the quality of the membrane and cytosolic extracts from Huh7, RD-U and RD-D cells, the localisation of the cytosolic protein lactate dehydrogenase (LDH) was examined by western blot (Figure 5-12 and Figure $5-13)$.

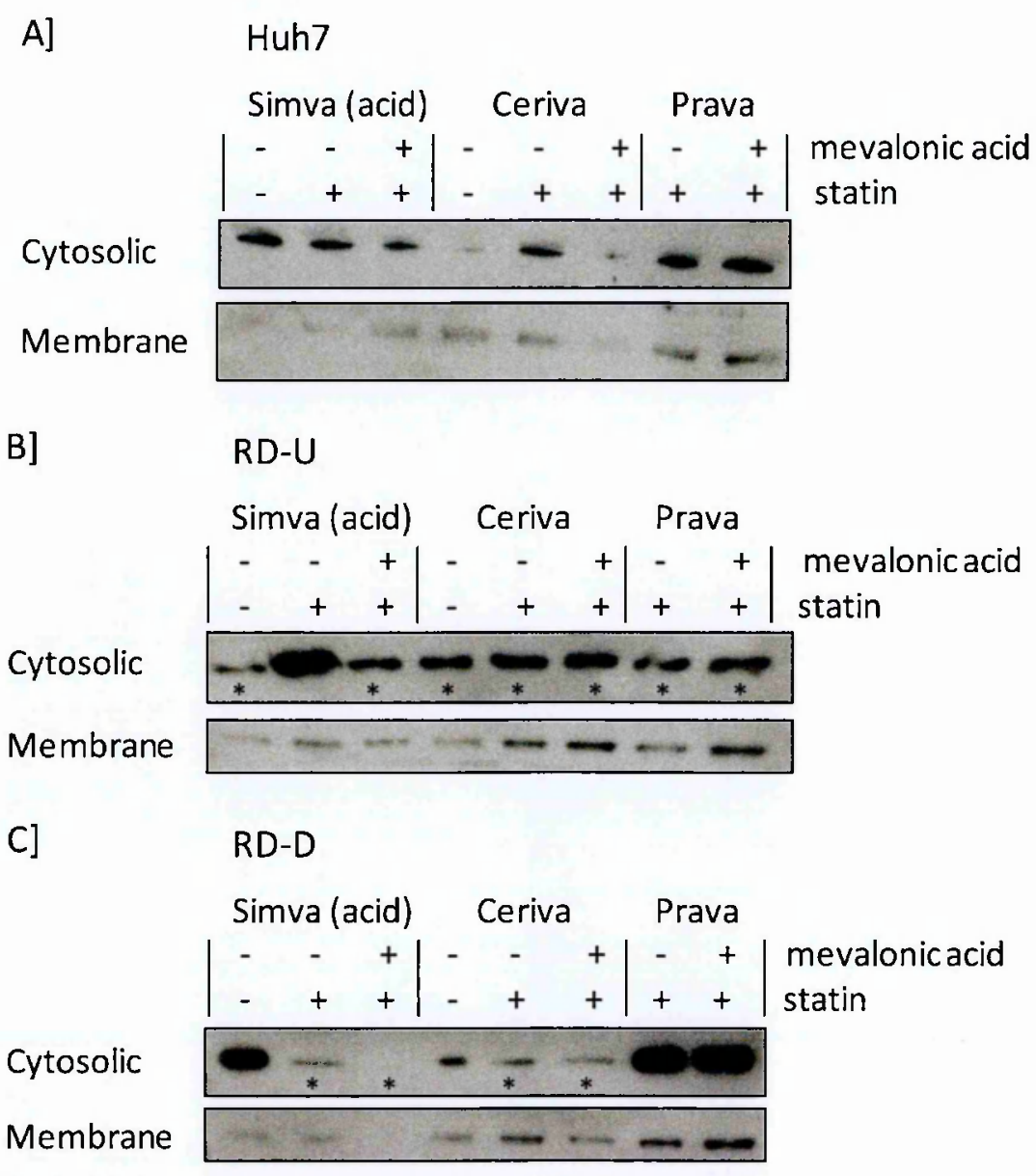

Figure 5-12 Localisation of lactate dehydrogenase (LDH), in response to statin treatment Fractions enriched with either cytosolic or membrane protein were extracted from cells treated for 48 hours with vehicle control, statin, or mevalonic acid in addition to statin, and $4 \mu \mathrm{g}$ was separated by SDS-PAGE. Simvastatin and pravastatin were used at a concentration of $10 \mu \mathrm{M}$ and cerivastatin at $1 \mu \mathrm{M}$. DMSO was used as a vehicle control for simvastatin and water for cerivastatin and pravastatin. Mevalonic acid was used in excess, at $100 \mu \mathrm{M}$. The level of LDH (35kDa) in each fraction was detected in $\operatorname{Huh} 7(A), R D-U(B)$ and RD-D (C) cells.

* Protein sample precipitated and resuspended prior to SDS-PAGE. 
Initially there were problems with the antibody that meant that it was not possible to examine LDH using the same membranes as used for previous analysis of H-Ras (Figure 5-6 and Figure 5-7), M-Ras (Figure 5-8 and Figure 5-9) and Rab1A localisation (Figure 5-10 and Figure 5-11). Therefore, a second set of protein extracts were generated, separated by SDS-PAGE and transferred to PVDF membrane for use in LDH analysis, with the aim of subsequently also blotting these membranes for $\mathrm{H}$-Ras, M-Ras and Rab1A as a repeat experiment.

In extracts from all cell types, on control treatment and treatment with both statins (Figure 5-12) and prenylation inhibitors (Figure 5-13) a much greater amount of LDH was observed in the cytosolic fraction than in the corresponding membrane fraction. A small amount of LDH remained in the majority of membrane fractions, indicating that the separation of cytosolic and membrane proteins achieved by this extraction method was not $100 \%$. This could be further examined by analysing the same samples for the presence of proteins known to be exclusively found in the membrane, for example membrane transporters, but unfortunately time constraints did not allow this in this instance.

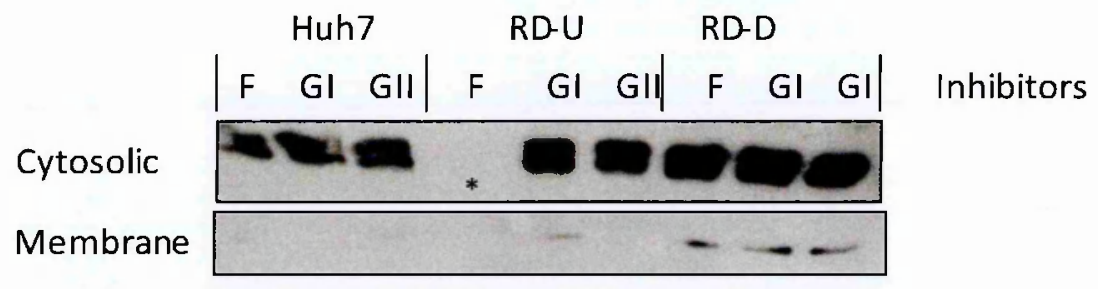

Figure 5-13 Localisation of LDH, in response to treatment with prenyltransferase inhibitors

Fractions enriched with either cytosolic or membrane protein were extracted from cells treated for 48 hours with $10 \mu \mathrm{M}$ farnesyltransferase inhibitor FTI-277 $(\mathrm{F}), \quad 10 \mu \mathrm{M}$ geranylgeranyltransferase-I inhibitor GGTI-2133 (GI) or 100 $\mu \mathrm{M}$ perillyl alcohol (GII), and $4 \mu \mathrm{g}$ was separated by SDS-PAGE. The level of LDH $(35 \mathrm{kDa})$ in each fraction was detected using a specific antibody. * Protein sample precipitated and resuspended prior to SDS-PAGE.

There appears to be some variation in the amount of LDH observed between protein samples. This could possibly be explained by the fact that some of the RD-U and RD-D samples (denoted * on Figure 5-12 and Figure 5-13) 
underwent protein precipitation and resuspension (section 2.2.3.4) due to the extracted protein not being concentrated enough for SDS-PAGE analysis. The samples that were not pre-treated in this way seem to show detection of greater amounts of LDH.

The difference between the amount of LDH in the cytosolic and membrane fractions is enough that any gross changes in subcellular localisation caused by reduced prenylation should still be observed using protein extracted using this method. However, due to the variability between samples, further analysis of $\mathrm{H}$ Ras, M-Ras and Rab1A was not carried out using these repeat membranes.

\subsection{The Effect of Statins on Prenyltransferase Expression}

The differences in prenylation observed on statin treatment could be due to either a change in the isoprenoids themselves, or in the expression of the prenyltransferase enzymes. In addition, it has been suggested that levels of prenyltransferase enzymes could vary between cell types, and that this could impact upon results observed (Ali et al. 2010). In order to rule out these possibilities, expression of each of the three prenyltransferase enzymes was measured, by western blot analysis, before and after treatment with statin or prenylation inhibitor.

FT and GGT-I are heterodimers that share a $48 \mathrm{kDa} \alpha$ subunit but have different $\beta$ subunits (46kDa and $42 \mathrm{kDa}$ respectively). GGT-II consists of distinct $\alpha$ $(60 \mathrm{kDa})$ and $\beta(40 \mathrm{kDa})$ subunits. It is thought that the $\beta$ subunits contain the substrate binding sites and that it is these different $\beta$ subunits which provide the different substrate specificities of these enzymes (Casey and Seabra 1996; McTaggart 2006). For this reason, it is necessary to use antibodies specific to the $\beta$ subunits rather than the alpha subunits. 
Expression of all three of these enzymes was confirmed in Huh7, RD-U and RD-D cells. There was no significant change in expression of FT, GGT-I or GGT-II in any of the cell types on treatment with statin alone or in combination with mevalonic acid (Figure 5-14 and Figure 5-15). The apparent decrease in GGT-II $\beta$ in RD-U cells on treatment with cerivastatin (Figure 5-15B) corresponds to a lower level also of $\beta$-actin in this sample, and so is not a real effect of cerivastatin. Therefore, the changes in prenylation state of Rap1A and the change in membrane association of $\mathrm{H}$-Ras, M-Ras or Rab1A in response to statin treatment cannot be explained by alteration in the expression level of the prenyltransferase enzymes.

A] Huh7

B]

RD-U

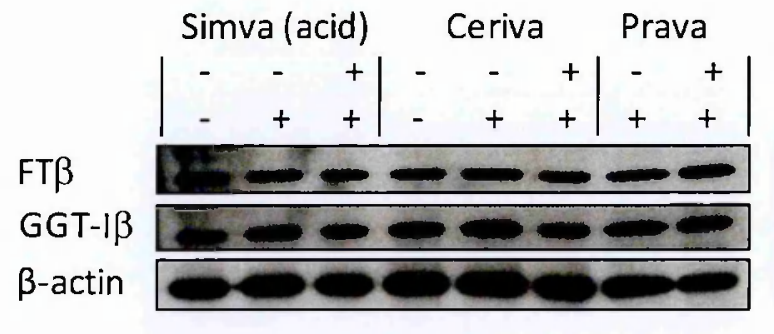
Simva (acid) Ceriva Prava

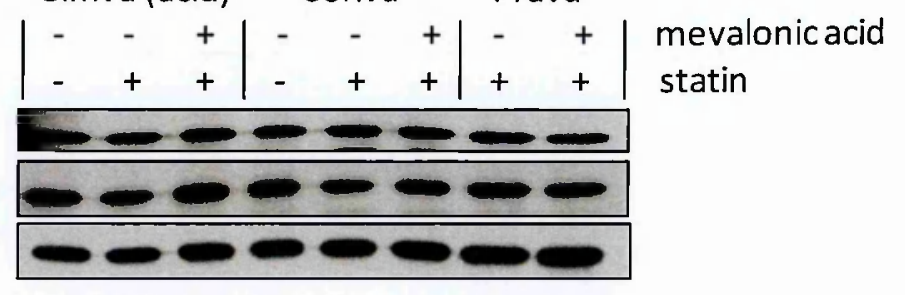

C]

RD-D

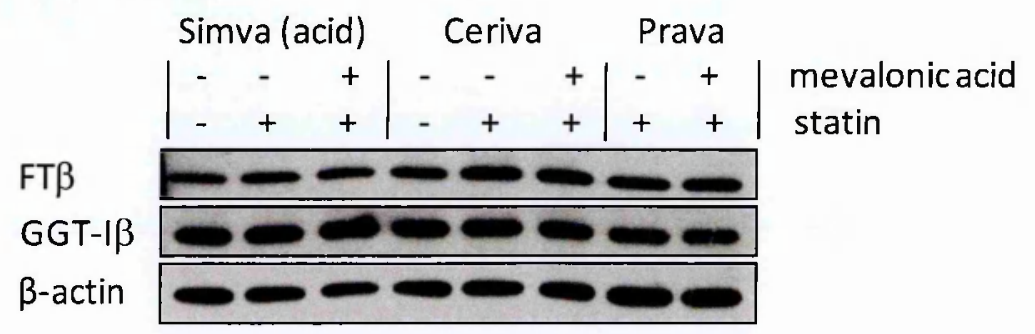

Figure 5-14 Effect of statin treatment on expression of farnesyltransferase and geranylgeranyltransferase-I

Total protein was extracted from cells treated for 48 hours with vehicle control, statin, or mevalonic acid in addition to statin, and $30 \mu \mathrm{g}$ was separated by SDS-PAGE. Simvastatin and pravastatin were used at a concentration of $10 \mu \mathrm{M}$ and cerivastatin at $1 \mu \mathrm{M}$. DMSO was used as a vehicle control for simvastatin and water for cerivastatin and pravastatin. Mevalonic acid was used in excess, at $100 \mu \mathrm{M}$. Expression of farnesyltransferase (46kDa) and geranylgeranyltransferase-I (42kDa) was detected in Huh7 (A), RD-U (B) and RD-D (C) cells using specific antibodies, and compared to the level of the loading control, $\beta$-actin. 


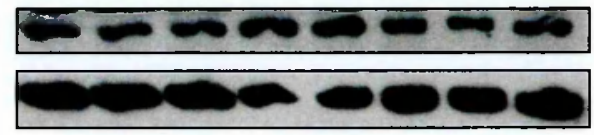

C] RD-D
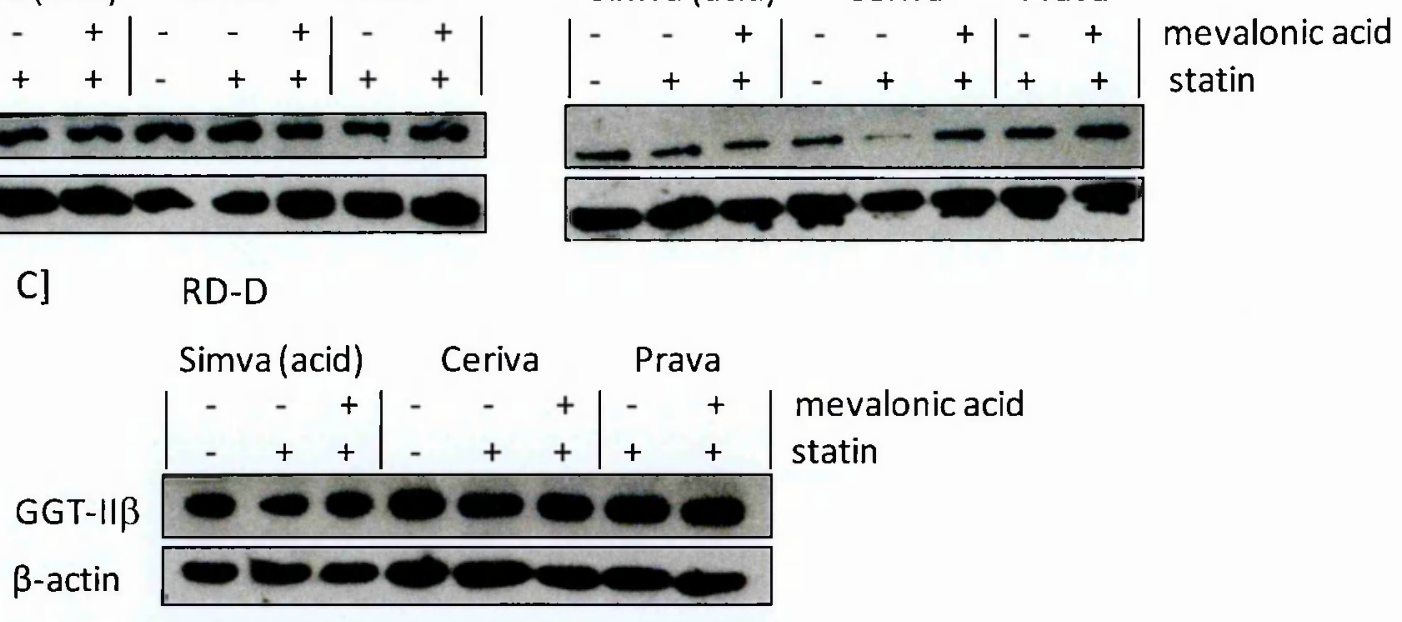

Figure 5-15 Effect of statin treatment on expression of geranylgeranyltransferase-II

Total protein was extracted from cells treated for 48 hours with vehicle control, statin, or mevalonic acid in addition to statin, and $30 \mu \mathrm{g}$ was separated by SDS-PAGE. Simvastatin and pravastatin were used at a concentration of $10 \mu \mathrm{M}$ and cerivastatin at $1 \mu \mathrm{M}$. DMSO was used as a vehicle control for simvastatin and water for cerivastatin (and pravastatin). Mevalonic acid was used in excess, at $100 \mu \mathrm{M}$. Expression of geranylgeranyltransferase-II (43kDa) was detected in Huh7 (A), RD-U (B) and RD-D (C) cells using a specific antibody, and compared to the level of the loading control, $\beta$-actin.

Similarly, treatment with prenyltransferase inhibitors also did not alter expression of FT, GGT-I or GGT-II (Figure 5-16). In addition, this analysis shows that there may be slightly higher levels of prenyltransferase expression in muscle compared to liver cells, although densitometry would be needed to confirm this: Regardless, any differences would not be enough to explain the differences in sensitivity to the disruption of prenylation between muscle and liver cells observed previously (section 5.3.1.1). To my knowledge, this is the first time that the expression levels of these enzymes have been compared between liver and muscle.

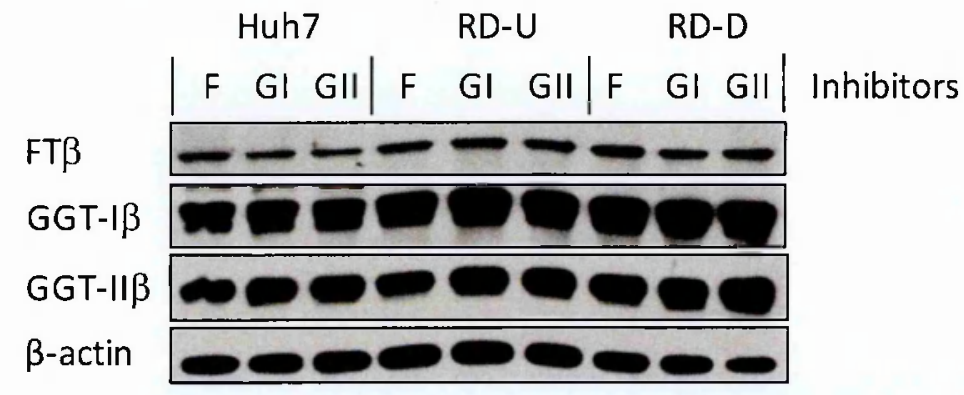

Figure 5-16 Effect of prenylation inhibitors on expression of farnesyltransferase, geranylgeranyltransferase-I and geranylgeranyltransferase-II

Total protein was extracted from Huh7, RD-U and RD-D cells treated for 48 hours with $10 \mu \mathrm{M}$

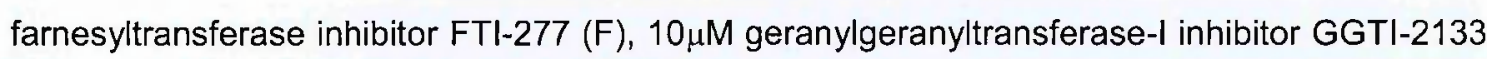
(GI) or $100 \mu \mathrm{M}$ perillyl alcohol (GII), and $30 \mu \mathrm{g}$ was separated by SDS-PAGE. Expression of farnesyltransferase $(46 \mathrm{kDa})$, geranylgeranyltransferase-1 $(42 \mathrm{kDa})$ and geranylgeranyltransferase-II (43kDa) was detected using specific antibodies, and compared to 


\subsection{Discussion}

\subsubsection{The Significance of Prenylation}

The post-translational modification of proteins by prenylation is of great physiological importance, with at least 100 proteins known to require this modification in order to carry out their role in a range of cellular processes (Lane and Beese 2006). This importance has been highlighted by the abnormalities observed in recently generated conditional knockout mice for the $\beta$ subunits of FT (Fntb) and GGT-I (Pggt1b), which therefore lack functional FT or GGT-I. Keratinocyte-specific Fntb knockout mice survived but developed severe hair loss, and cells from these mice could not proliferate in culture, whereas keratinocyte-specific Pggt1b knockout mice died shortly after birth (Lee et al. 2010).

The small GTPases represent the largest group of prenylated proteins. They are a large class of cell-signalling proteins that have important roles in the regulation of cellular proliferation, gene expression, cytoskeleton organisation, cell motility and intracellular vesicle trafficking (Cordle et al. 2005; Matozaki et al. 2000). Therefore, disruption of the prenylation of small GTPases can have wide-ranging physiological consequences, and has been postulated to play a role in statin-induced myopathy.

\subsubsection{Prenylation and the Statins}

There is a growing body of evidence supporting the idea that statins can disrupt prenylation of small GTPases, resulting in the disruption of normal cellular signalling. Such evidence includes the observation that inhibition of GGT-I and II, with perillyl alcohol treatment, has been shown to mimic the effect of fluvastatin on isolated rat skeletal myofibres, with these effects being rescued by co-addition of GGPP (Sakamoto et al. 2007). Treatment of rat skeletal myoblasts with cerivastatin has been shown to result in the activation and detachment of membrane-bound Ras, leading to apoptosis (Matzno et al. 2005). Statins have been shown to induce apoptosis in vitro in a range of different cells (Dirks and Jones 2006); for example, treatment of rat vascular smooth muscle cells with atorvastatin or simvastatin in vitro resulted in 
apoptosis that could only be rescued by addition of mevalonate, FPP or GGPP (Blanco-Colio et al. 2002).

However, much of the previous research has been carried out only in muscle and often in non-human tissue. It is therefore important to ensure that these effects are similarly observed in human cells. The analysis here also aimed to compare the effects in liver (the target tissue) with muscle (the main site of toxicity) across a range of statins, as it is thought that any differences observed between the responses of muscle and liver may give us further insight into the mechanism of statin muscle toxicity (Mullen et al. 2010). The hypothesis to be tested was that prenylation, postulated to play a role in statin-induced myopathy, would show preferential disruption in muscle compared to liver.

\subsubsection{Membrane Association of Small GTPases}

The results described in this chapter confirm that statin treatment does have an effect on the prenylation of small GTPases in both human liver and muscle cells. On cerivastatin treatment, detection of the FT substrate H-Ras, GGT-I substrate M-Ras and the GGT-II substrate Rab1A in the membrane protein fraction was greatly reduced in both human muscle and liver cells. Since prenylation is vital for the membrane association of small GTPases, this change in subcellular localisation indicated a reduction in prenylation.

In some cases, the reduced membrane-association was accompanied by detection of the protein in the corresponding cytosolic fractions, which could sometimes also be seen with simvastatin treatment. It is interesting that this corresponding cytosolic increase was not observed in all instances. It could perhaps be that the unprenylated protein is targeted for degradation, which would explain why it could not always be detected.

It is also interesting to note that these effects on prenylation were only observed on treatment with lipophilic cerivastatin or simvastatin. Treatment with hydrophilic pravastatin, which was previously shown to elicit lower levels of toxicity (section 4.2), did not lead to a reduction in membrane-association under the conditions used. 
Since the reduction in membrane-associated (i.e. prenylated) proteins was observed both in liver and muscle cells, it would be beneficial to further dissect this inhibition of $\mathrm{H}$-Ras, M-Ras and Rab1A prenylation. This could be achieved through the extraction of cytosolic and membrane protein from cells treated with a range of simvastatin and cerivastatin doses, as was previously carried out during the examination of Rap1A prenylation using total protein extracts (section 5.3.1.1).

A limitation of this data is the lack of complete confidence in the quality of cytosolic and membrane separation in those samples used to look at $\mathrm{H}$-Ras, $\mathrm{M}$ Ras and Rab1A localisation, in terms of the degree of separation obtained. LDH is exclusively a cytosolic protein (Saad et al. 2006), and so the amount of the protein present in cytosolic, compared to membrane, extracts was examined to act as a control and give an indication of the degree of separation achieved by the extraction process used. However, the results obtained were inconclusive due to variations within the particular set of protein samples used in this analysis (Figure 5-12 and Figure 5-13). This was likely the result of the protein concentration, by precipitation and resuspension in a smaller amount of solvent, in a number of these samples prior to analysis. The protein was resuspended in sodium hydroxide, which allowed for the determination of final protein concentration using the Lowry method but was later found to interfere with SDS-PAGE. This meant that the sodium hydroxide needed to be neutralised prior to analysis (section 2.2.3.4), which may have affected the subsequent separation of proteins by gel electrophoresis. A repeat analysis of LDH localisation, using samples that had not been pre-treated in this way or had been resuspended in a solvent other than sodium hydroxide, may rectify this. Time did not allow in this case, however it would be beneficial to also analyse the localisation of a membrane protein in the different protein fractions. This could include the analysis of a membrane-specific protein, such as calnexin (Spindler et al. 2012), which has been shown not to be affected by statin treatment (Ostrowski et al. 2007). Finally, measurement of the expression level of the membrane transporter OATP2B1 would be beneficial, as it is known to be expressed in both liver and skeletal muscle (Knauer et al. 
2010) and is responsible for the uptake of hydrophilic statins into cells (section 1.1.4).

\subsubsection{Differential Disruption of Prenylation}

In addition to the effect on small GTPase localisation, a direct effect was observed on the prenylation of the Ras-related GTPase Rap1A through the use of two antibodies able to detect the unprenylated form of the protein or total protein expression respectively. Unprenylated Rap1A was detected in both liver and muscle following simvastatin and cerivastatin treatment. This inhibition of Rap1A prenylation was dose-dependent in both cases with all cells showing much greater sensitivity to cerivastatin than simvastatin, with lower cerivastatin concentrations required to produce detectable levels of unprenylated Rap1A.

The differences between the responses to simvastatin and cerivastatin treatment are unlikely to be dependent on differential uptake of the two statins. These are two of the most lipophilic statins, and they both show distribution to non-target tissues via passive diffusion. They are also both substrates of OATP1B1 (section 1.1.4), a transporter predominantly found in the liver which plays an important role in the hepatic uptake of statins (Shitara and Sugiyama 2006).

RD muscle cells were more sensitive to treatment with both simvastatin and cerivastatin compared to Huh7 liver cells, with a much lower statin concentration needed to detect unprenylated Rap1A in muscle. This finding supports the hypothesis, that statin treatment does lead to both greater toxicity and preferential disruption of protein prenylation within muscle cells.

It is unlikely that this difference between muscle and liver is due to increased statin metabolism in Huh7 liver cells. The liver is the main site of xenobiotic metabolism in vivo (Caldwell et al. 1995), however it has been shown that in vitro Huh7 liver cells express very low levels of drug metabolising enzymes (Phillips et al. 2005). This could mean that the differential effect seen between muscle and liver in vivo may be even greater. 


\subsubsection{Prenyltransferase Inhibitors}

Treatment of cells with prenyltransferase inhibitors resulted in disruption of protein prenylation, as observed through detection of unprenylated Rap1A and reduction of $\mathrm{H}-\mathrm{Ras}, \mathrm{M}-\mathrm{Ras}$ and Rab1A found in membrane protein fractions. This was similar to effects seen with simvastatin and cerivastatin treatment in these experiments, and agrees with previous findings of similar effects of statin and prenylation inhibitor treatment in other models (Cao et al. 2009; Sakamoto et al. 2007; Wagner et al. 2011). This supports the idea that statins are affecting the process of protein prenylation.

Treatment with the GGT-I inhibitor GGTI-2133 resulted in observation of unprenylated GGT-I substrate Rap1A in all cell types (Figure 5-3). However, it is surprising that although $\mathrm{H}-\mathrm{R}$ as and Rab1A are reported to be substrates of different prenyltransferase enzymes (FT and GGT-II respectively), on treatment with prenyltransferase inhibitors the pattern of their reduced membraneassociation was similar. For both small GTPases, treatment with GGTI-2133 resulted in loss of membrane association in Huh7 liver cells, but in RD muscle cells this was observed on treatment with the FT inhibitor FTI-277.

Under normal conditions H-Ras is reputed to undergo farnesylation by FT. However, it is known that $\mathrm{K}$ - and $\mathrm{N}$-Ras proteins are alternately prenylated by GGT-I in the human colon carcinoma cell line DLD-1 on treatment with FT inhibitors (Whyte et al. 1997). It is perhaps possible that when FT is inhibited in vitro in liver cells then H-Ras can similarly be alternately prenylated by GGT-I. This would explain why treatment of Huh7 cells with FTI-277 did not result in a decrease in membrane-associated H-Ras. In addition, GGTI-2133 can also inhibit FT, as described previously (section 5.2), but with lower potency than for GGT-I inhibition. The $\mathrm{IC}_{50}$ for GTI-2133 inhibition of FT is reported to be $5.4 \mu \mathrm{M}$ (Sigma-Aldrich 2011b), and hence the $10 \mu \mathrm{M}$ inhibitor concentration used herein should result in approximately $65 \%$ inhibition of activity. Therefore, the observed reduction of membrane-associated $\mathrm{H}$-Ras on treatment of Huh7 cells

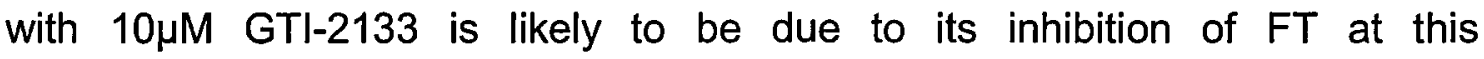
concentration, although this does not explain why this effect is seen in liver cells 
and not also in muscle cells. Another possibility is that the FT inhibitor used here is inhibiting GGT-Il in the RD cell line, as has been previously observed in Caenorhabditis elegans (Lackner et al. 2005; McTaggart 2006). This would explain the reduction in Rab1A membrane-association in RD cells treated with FT inhibitor. Perhaps the similar expression profiles of H-Ras and Rab1A indicate that either the prenyltransferase enzymes, or these prenyltransferase inhibitors, are not as specific under all conditions as traditionally thought.

Future repeat experiments, perhaps with a range of lower inhibitor concentrations, would help to investigate these discrepancies and also help to rule out any possibility of having mixed up samples. Lower concentrations of GGTI-2133 will be less likely to have a possible effect on prenylation by FT, (IC $\left.C_{50} 5.4 \mu \mathrm{M}\right)$ but will still inhibit GGT-I (IC 50 38nM) (Sigma-Aldrich 2011b). Similarly, the use of lower concentrations of FTI-277 may help to clarity whether or not FTI-277 inhibition of GGT-II was the reason for observing loss of membrane association of Rab1A in muscle on treatment with this inhibitor.

\subsubsection{Prenyltransferase Expression}

No change in the expression levels of any of the 3 prenyltransferase enzymes was observed, in either liver or muscle cells, in response to treatment with statins or prenyltransferase inhibitors. If any of these enzymes are upregulated or downregulated in response to statin inhibition of prenylation, then it would be expected to have occurred after the given treatment time of 48 hours. The fact that no change was observed after this time excludes this as an explanation of the effects of statin treatment on prenylation or as an explanation of the differences between the responses to simvastatin and cerivastatin. There were also no differences in enzyme levels between the cell types that would account for the different sensitivities of liver and muscle to disruption of protein prenylation. Therefore, several alternative explanations for this differential sensitivity will be discussed in the final chapter. 


\subsection{Summary}

The post-translational modification of small GTPases by prenylation plays a vital role in enabling their membrane localisation in order to carry out their varied roles in cellular signalling. It is thought that statins inhibit not only the production of cholesterol but also the production of the isoprenoids FPP and GGPP which are used to prenylate proteins. It has therefore been suggested that statins inhibit the prenylation of proteins and that this is an important contributing factor in the development of statin-induced muscle toxicity.

The results presented here confirm that treatment with both simvastatin and cerivastatin does inhibit the prenylation of a range of small GTPases in both human liver and muscle in a similar way to the inhibition of prenylation observed on treatment with prenyltransferase inhibitors.

The inhibition of prenylation of Rap1A, a Ras-related GTPase, was more sensitive to treatment with cerivastatin than simvastatin in both muscle and liver. Inhibition of Rap1A prenylation was also more sensitive to statin treatment in muscle (the tissue where the most toxicity is observed) than in liver (the target tissue). These effects could not be explained by changes in total protein expression. This supports my hypothesis, that statin treatment does lead to both greater toxicity and preferential disruption of protein prenylation within muscle cells.

There was no change observed in expression of any of the three prenyltransferase enzymes in liver or muscle in response to treatment with statins or prenylation inhibitors. The changes in prenylation status of Rap1A, or any of the other small GTPases examined, therefore cannot be explained by changes in enzyme expression. 


\section{Discussion}

The physiological consequences of xenobiotic intervention are often complex. A drug frequently has many effects in addition to the intended ones. These can either benefit the patient or can lead to adverse drug reactions, depending on the treatment context and on the individual patient. This highlights the ever increasing need to understand the in-depth mechanisms of drug action, in order to improve patient treatment both through enabling the best used of established medication as well as directing the future design of new drugs.

The statins are an important class of drugs used to treat hypercholesterolaemia for the primary and secondary prevention of cardiovascular disease, currently the leading cause of death worldwide. They are widely used, with their use continually increasing, however a small percentage of patients experience drug side effects; the most significant of these is myopathy, which can range from the muscle aches or weakness of myalgia to the more serious condition of rhabdomyolysis (Harper and Jacobson 2007). In addition to the obvious adverse effects for the patients myopathy is also considered a leading cause of the high statin discontinuation rates (Bakhai et al. 2012), and therefore it is important to understand the mechanism of this myopathy to enable better management of statin treatment. It is currently thought that myopathy occurs due to the secondary effects resulting from statin inhibition of 3-hydroxy-3methylglutaryl coenzyme A reductase (HMGCR), the rate limiting enzyme in cholesterol biosynthesis and the mevalonate pathway (Shitara and Sugiyama 2006). By inhibiting this enzyme, statin treatment lowers cholesterol in the form of low-density lipoprotein (LDL) but can also disrupt other aspects of the mevalonate pathway, such as protein prenylation. It is this disruption of protein prenylation that has been postulated to play a particular role in statin-induced myopathy, however the mechanism has not yet been fully elucidated (Vaklavas et al. 2009).

The aim of this study was to use two human cell lines as an in vitro model for comparing the effects of statins on both cholesterol synthesis and protein 
prenylation between the liver, the intended target tissue for therapeutic benefit, and skeletal muscle, the major site of toxicity.

\subsection{In Vitro Modelling of Statin-Induced Toxicity}

Initially, the suitability of the RD rhabdomyosarcoma skeletal muscle cell line for use in this model system was determined (section 3.2). The morphological and molecular changes that occur during the differentiation process of these cells were examined. RD cells were found to express a number of muscle-specific markers, including the muscle-specific transcription factor myogenin and myosin heavy chain 3, as well as a number of other genes of interest such as HMGCR and the LDL receptor. On the basis of this investigation, a cell differentiation period of 5 days was chosen for use in later experiments.

The original hypothesis to then be tested was that the process of protein prenylation exhibits circadian variation, as has already been shown for cholesterol biosynthesis, and that this might enable temporal separation between the cholesterol-lowering efficacy and the toxicity of statins. However, being unable to verify the ability of the RD cell line to establish and maintain a circadian cycle in vitro (section 3.3), the project took a slightly different direction, whilst still focusing on examining the differences between the responses of liver and muscle to statin treatment.

In order to investigate any such differences, a number of parameters were examined. MTT assays were carried out in order to examine the cell toxicity response of Huh7 liver and RD muscle cells to treatment with a range of statins (section 4.2). Significant reductions in cell viability were observed in all cells on statin treatment, with greater toxicity observed in response to treatment with more lipophilic statins, such as simvastatin, lovastatin and cerivastatin. Where it was possible to determine IC50 values, these were consistent with those previously reported by Kobayashi and colleagues using RD cells (Kobayashi et al. 2008). Greater toxicity was also observed in RD muscle cells compared to Huh7 liver cells on treatment with all statins, with this difference being statistically significant for treatment with simvastatin, cerivastatin, atorvastatin and rosuvastatin. Previous studies examining the toxicity of the statins have 
tended to either compare the toxicity of a large range of statins in only one tissue, such as muscle (Kobayashi et al. 2008), or to compare the responses between liver and muscle using only a small number of statins (Mullen et al. 2010; Mullen et al. 2011). Therefore, since this study both compared responses between liver and muscle cell lines and used a large range of different statins, it represents the first comprehensive comparative analysis of statin toxicity.

The process of cholesterol synthesis was then investigated in these cells (section 4.3). Much lower expression of HMGCR was found, at both the transcript and protein level, in RD cells compared to Huh7 cells. This difference was particularly evident in differentiated RD cells. Cholesterol was found to be present in both Huh7 and RD cells, with $10 \mu \mathrm{M}$ simvastatin and pravastatin treatment, and $1 \mu \mathrm{M}$ cerivastatin treatment, seeming to lead to a decrease in total cellular cholesterol in these cells. This cholesterol decrease was observed after 48 hour statin treatment, which agrees with previous observations of a cholesterol decrease after 24 and 48 hour treatment of HepG2 cells (Kobayashi et al. 2008) but not after 6 or 18 hour treatment of HepG2 cells and C2C12 mouse myoblasts (Mullen et al. 2010).

Having characterised the response of Huh7 and RD cells to statin treatment, in terms of cell viability and changes in cholesterol levels, experiments were designed to test the hypothesis that statins preferentially disrupt the process of protein prenylation within RD muscle cells (chapter 5), the site of greater toxicity. Huh7 and RD cells were treated with simvastatin, cerivastatin and pravastatin, mevalonic acid in addition to statin, or prenylation inhibitors. Protein was extracted and then subjected to analysis by western blot to detect any changes in the prenylation status of selected small GTPases. Inhibition of prenylation was found by directly analysing changes in the unprenylated form of Rap1A, using a specific antibody, and by examining the reduction in membrane association of $\mathrm{H}$-Ras, M-Ras and Rab1A. Treatment with simvastatin and cerivastatin inhibited the prenylation of Rap $1 \mathrm{~A}$, and frequently seemed to also affect the membrane association of the small GTPases H-Ras, M-Ras and Rab1A. These effects could not be explained by changes in total protein expression or changes in the expression of the prenyltransferase enzymes. 
In both muscle and liver the inhibition of prenylation of Rap1A, a Ras-related GTPase, was more sensitive to treatment with cerivastatin, the statin that was withdrawn from the market in 2001 due to high incidence of rhabdomyolysis (Bellingham 2001), than simvastatin. Inhibition of Rap1A prenylation in muscle cells was also more sensitive to simvastatin and cerivastatin treatment than in liver cells (section 5.3.1.1). This supports my hypothesis, that statin treatment does lead to both greater toxicity and preferential disruption of protein prenylation within muscle cells and further supports a mechanism where disrupting production of intermediates in the mevalonate pathway is indeed a cause of statin-induced muscle toxicity.

A question arising from this conclusion is that of why there is this differential sensitivity between liver and muscle cells to the inhibition of prenyiation by statins. One possible explanation could be that there is a lower background level of prenylation occurring in muscle compared to liver. This is unlikely to be due to differences in the levels of the prenyltransferase enzymes, since no large differences in expression were observed between the cell types (Figure 5-16); although this analysis only examined protein expression and did not investigate whether there were differences in prenyltransferase enzyme activity. However, the results did reveal a considerable difference in HMGCR expression, at both the transcript and the protein level, between Huh7 liver and RD muscle cells (section 4.3.1). This implies that there may be a much lower throughput along the mevalonate pathway in muscle when compared to liver, the main site of cholesterol synthesis (Catapano 2007). In the absence of statin treatment, there are sufficient isoprenoids in both liver and muscle cells to ensure complete prenylation of Rap1A, as far as can be detected by the western blot analysis; but a lower capacity of the mevalonate pathway could lead to a smaller resting pool of the isoprenoids FPP and GGPP within muscle and would mean that less inhibition of this pathway would be required before the effects on prenylation would be seen in this tissue. In order to examine this as a possibility, it would be interesting to measure and compare the levels of FPP and GGPP, under resting conditions, in both liver and muscle, initially in the cell lines that were used here. This could be achieved by using a combination of HPLC (high performance liquid chromatography) and LC-MS (liquid 
chromatography - mass spectrometry)-based techniques (Tong et al. 2005). This method could also be used for looking at the rate at which a range of statins decrease this isoprenoid pool in both liver and muscle, both over time and on treatment with varying statin concentrations.

\subsection{Prenylation and Muscle Physiology}

A second question is how this differential sensitivity of muscle to disruption of protein prenylation could lead to the toxicity seen in muscle. There is the possibility that prenylation, or at least the prenylation of certain proteins, may be more obligate in muscle than in liver. Perhaps there are certain prenylated proteins that are more highly expressed in muscle than in liver or are particularly crucial for correct muscle function. M-Ras, initially thought to be such a target, is now known to be of more widespread importance, and so cannot now be included in this category (Ehrhardt et al. 1999). However if such targets do exist, and their prenylation status is affected by statin treatment, then they could play an important role in statin muscle toxicity.

In the past, knockout models have frequently been used to elucidate the physiological importance of a target protein or pathway; however knowledge gained in this way regarding protein prenylation remains limited. Recently, several conditional knockouts of prenyltransferase enzymes have been generated and studied, which is starting to fill this gap in knowledge: Conditional knockout of the $\beta$ subunit of FT in mouse keratinocytes led to development of severe hair loss and an inability of these cells to proliferate in culture, whereas knockout of the GGT-I $\beta$ subunit led to the death of mice shortly after birth (Lee et al. 2010), highlighting the essential role of protein prenylation. Similar deficiencies in either FT or GGT-I in hepatocytes led to elevated plasma transaminases and abnormal histology of livers from the knockout mice, with FT knockout mice also growing slower than wild-type mice and GGT-I knockout mice showing reduced survival (Yang et al. 2012). In addition, conditional knockout of GGT-I has been shown to lead to hyperactivated mouse macrophages, which secreted inflammatory cytokines and induced an inflammatory response (Khan et al. 2011; Philips 2011). Other recently generated models include the conditional knockout of GGT-I in mouse 
haematopoietic cells (Sjogren et al. 2011), knockdown of prenyltransferase enzymes in zebrafish embryos (Cao et al. 2009), knockout mouse models of several Rho family GTPases (Heasman and Ridley 2008; Ueda et al. 2010) and mouse knockout of the skeletal muscle-specific $G$ protein $\alpha$ subunit (Chen et al. 2009a). Further study, particularly of these latter models, as well as the generation of muscle-specific conditional prenyltransferase knockout or knockdown models, may assist in the elucidation of the specific role of protein prenylation in muscle.

Meanwhile, in order to link the molecular disruption caused by statins to muscle toxicity we can gain clues through alternative in vitro studies, in many of which statin treatment has been linked with the apoptosis of muscle fibres, and through the identification of syndromes which are associated with muscle disease (reviewed by (Baker 2005)). Many such studies have been described in previous chapters but some of these findings, and the resulting proposed links between statin treatment and muscular effects, are now summarised in Figure 6-1.

Statin treatment has been shown to affect many aspects of the mevalonate pathway in vitro, which in turn has the potential to affect a wide range of cellular processes (Figure 6-1). Statins have been shown to cause a reduction in isopentylation of sec tRNA and therefore reduce selenoprotein synthesis (Warner et al. 2000). There are approximately 25 human selenoproteins, but out of these selenoprotein N (Jurynec et al. 2008; Petit et al. 2003) and glutathione peroxidase (Kromer and Moosmann 2009) have been identified as having a potential role in linking the statins to effects on muscle physiology. Statin treatment has reduced synthesis of dolichol and thereby disrupted $\mathrm{N}$ linked glycosylation (Mullen et al. 2010; Siddals et al. 2004) of proteins known to have an important role in normal muscle function (Barton et al. 2012). Statins have also been shown to disrupt the process of protein prenylation (Sakamoto et al. 2011), through either farnesylation or geranylgeranylation, which is known to affect cellular signalling via small GTPases and lead to muscle cell apoptosis (Blanco-Colio et al. 2002; Matzno et al. 2005). 


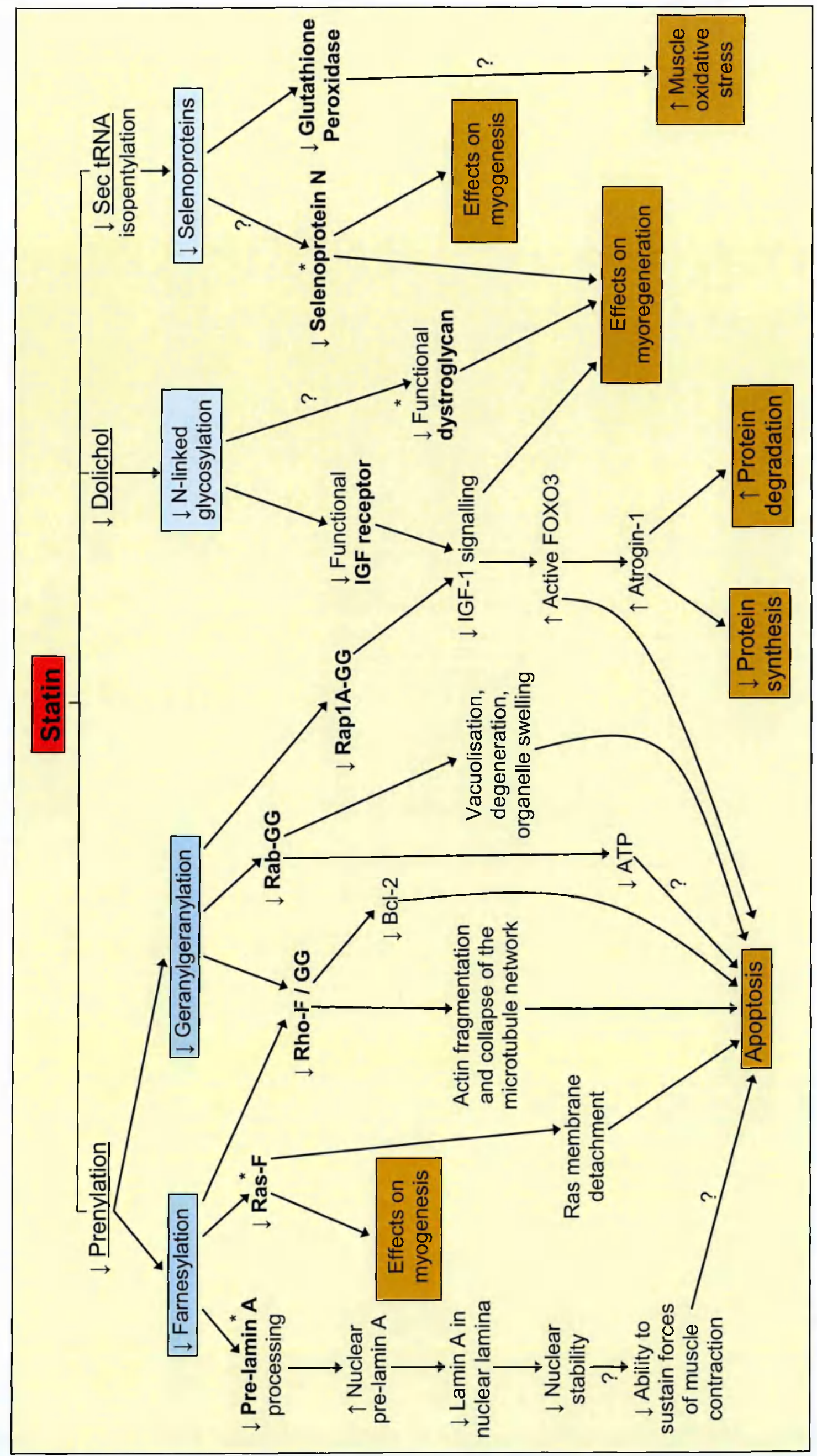

$\stackrel{0}{+}$ 


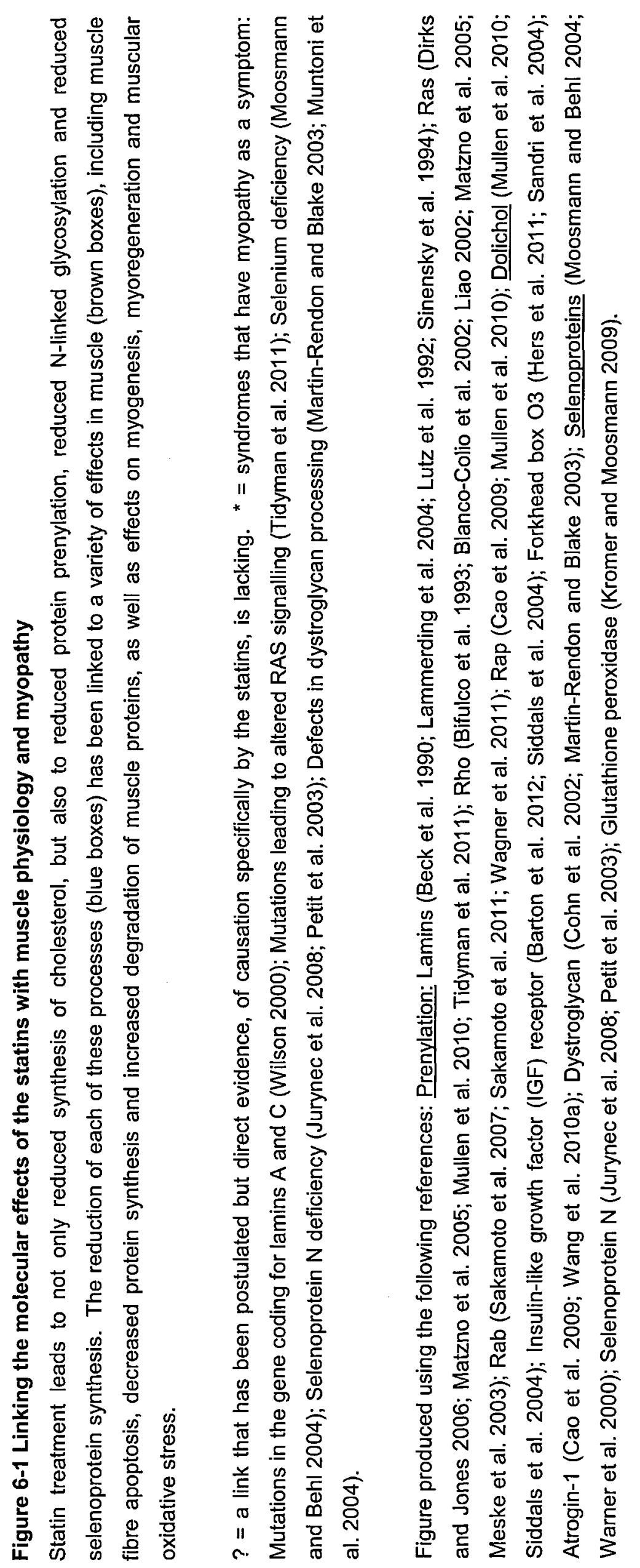


There are therefore many potential explanations for statin myopathy; however one pathway that has been emerging in recent years as having a particularly important role is the phosphatidylinositol 3-kinase (PI3K) - Akt signalling pathway. Insulinlike growth factor 1 (IGF-1) signalling, via this pathway, is known to have an important role in the regulation of skeletal muscle growth (Schiaffino and Mammucari 2011) but this signalling can be suppressed by the statins (Siddals et al. 2004). In addition, simvastatin treatment has been shown to lead to dephosphorylation of Akt in C2C12 mouse myotubes, but not in HepG2 liver cells, and co-treatment with simvastatin and IGF-1 has been able to rescue $\mathrm{C} 2 \mathrm{C} 12$ myotubes from apoptosis (Mullen et al. 2011). Suppression of normal signalling through the PI3K-Akt pathway leads to the expression of the muscle specific Skp1Cul1-F-Box protein (SCF) ubiquitin E3 ligase atrogin-1 (Sacheck et al. 2004) through the dephosphorylation, nuclear translocation and activation of the transcription factor FOXO3 (Sandri et al. 2004); it has now been shown that statin treatment can lead to the activation of atrogin-1 through this same pathway of FOXO3 activation (Cao et al. 2009).

It has been suggested that statins suppress IGF-1 signalling through two mechanisms. Firstly, statins have been shown to affect the $\mathrm{N}$-linked glycosylation and processing of IGF receptors (Siddals et al. 2004). Incorrectly processed IGF receptor could lead to impaired response to IGF growth factors in muscle cells.

Secondly, the inhibition of geranylgeranylation by statins has been shown to promote both upregulation of atrogin-1 and muscle damage in mouse myotubes and zebrafish, with myoblasts from atrogin-1 knockout mice proving resistant to this statin-induced muscle damage. This has led to the suggestion that there may be small GTPases that couple statin disruption of prenylation to the upregulation of atrogin-1 and muscle damage (Cao et al. 2009). The small GTPase Rap1, which is prenylated by geranylgeranyltransferase-I (GGT-I), has been suggested as an attractive candidate for this link (Cao et al. 2009; Mullen et al. 2011). This is because it is thought to play a role in IGF-1 signalling, as it can be activated by 
IGF-1 (Romano et al. 2006) and can then activate PI3K and Akt (Stork 2003; Van Kolen et al. 2006; Wang et al. 2001), and its prenylation can also be disrupted by the statins. This is further supported by the recent finding that transient transfection of constitutively active Rap1 into $\mathrm{C} 2 \mathrm{C} 12$ myotubes was able to partially rescue simvastatin-induced inhibition of mitochondrial respiration (Mullen et al. 2011). Rap1A, one of two very closely related isoforms of Rap1 (Bos 1997; Frische and Zwartkruis 2010), showed greater sensitivity to disruption of its prenylation in muscle compared to liver cells in my study (section 5.3.1.1). Therefore, the inhibition of Rap1 prenylation as a mechanism by which statins can suppress IGF-1 signalling would offer a potential interpretation for the relevance of my findings to statin-induced myopathy, however further investigation would be required to clarify this.

The suppression of IGF-1 signalling by both of these mechanisms ultimately results in the upregulation of atrogin-1, which is known to play an important role in muscle atrophy. The importance of atrogin-1 in muscle atrophy was first identified through the use of microarray experiments when its expression was found to be strongly upregulated in atrophying muscle, with this upregulation being prior to detection of muscle weight loss (Gomes et al. 2001). It has since been discovered to both stimulate degradation of muscle proteins and inhibit protein synthesis (Wang et al. 2010a). Potential degradation targets of atrogin-1 in muscle include myogenic differentiation 1 (MyOD1), the calcium-dependent phosphatase calcineurin, the translation initiation factor elF3-f, and intermediate filament components desmin and vimentin (Cao et al. 2009; Lokireddy et al. 2012). Degradation of these proteins would affect multiple processes that are essential for muscle cell function; such as differentiation of muscle satellite cells and thereby myoregeneration (MyoD1, calcineurin) (da Costa et al. 2007; Friday et al. 2000; Megeney and Rudnicki 1995); regulation of muscle fibre remodelling (calcineurin) (Bassel-Duby and Olson 2006; da Costa et al. 2007); synthesis of new proteins (elF3-f) (Csibi et al. 2008); and the integrity of the muscle cytoskeleton (desmin, vimentin) (Favre et al. 2011). 


\subsection{Additional Future Work}

In this study, only the prenylation of small GTPases in liver and muscle has been examined in response to statin treatment; however, there are a number of other proteins that have also been proposed to have a role in mediating statin-induced muscle toxicity, indicating that multiple pathways may yet be important for this adverse effect. Therefore, future work could include similar western blot analysis of non-GTPase proteins in liver and muscle that are known to be prenylated and whose dysfunction has been implicated in muscular dystrophy, indicating their importance in muscle; such proteins would include the nuclear lamins (Lutz et al. 1992; Wilson 2000). Additional mechanistic insight could be gained through further use of enzyme inhibitors and through the use of inhibitory RNA, to clarify whether changes in the extent of prenylation, as measured by western blot analysis, translate to an impact upon muscle cell integrity.

It would also be of interest to examine the differences between liver and muscle in a wider range of cellular processes, in response to a range of statins. For example, defective glycosylation has also been linked to muscular dystrophy (Muntoni et al. 2004), and it has previously been shown that simvastatin treatment affects $\mathrm{N}$-linked glycosylation in $\mathrm{C} 2 \mathrm{C} 12$ mouse myoblasts but not in human HepG2 liver cells (Mullen et al. 2010). However, it is not known if this would be the case in human muscle cells, such as the RD cell line used here, and whether there would be any differences between the responses to different statins. There are also many specific proteins that have been postulated to play a role in the mechanism of muscle toxicity where direct evidence is lacking. Such proteins include the glycoprotein dystroglycan and selenoprotein $\mathrm{N}$ and further investigation of their possible involvement, including differences between liver and muscle, may help to further clarify the mechanism of statin-induced muscle toxicity.

Finally, the issue of the contribution of circadian rhythms towards the development of statin-induced toxicity, as per my original hypothesis and objectives (section 1.5), remains an interesting question, albeit one which I was unable to fully 
investigate here due to the limitations of the model system used. Additional future work in this area of muscle toxicity could therefore include a return to this initial hypothesis using a different model system, such as the mouse $\mathrm{C} 2 \mathrm{C} 12$ myoblast cell line which, unlike the RD cell line, has previously been used for the analysis of the molecular clock in skeletal muscle (Zhang et al. 2012). Furthermore, since animal models have previously been used to analyse circadian variations in both liver (Akhtar et al. 2002) and skeletal muscle (Almon et al. 2008; McCarthy et al. 2007) in vivo, they could also be used as a starting point to examine the rhythmicity of protein prenylation in these tissues. The ubiquitous nature of circadian regulation, and the implication for xenobiotic toxicity, is increasingly being recognised as important (Gachon and Firsov 2011; Ohdo 2010) and therefore it is vital that we continue to develop model systems which can be used to investigate the potential applications for specific drugs, such as the statins.

\subsection{Summary}

In summary, this work has shown that, compared to Huh7 liver cells, RD muscle cells show both greater sensitivity to the toxic effects of statins and preferential disruption of protein prenylation. This further supports a mechanism whereby disrupting production of the intermediates in the cholesterol synthesis pathway, including the isoprenoids used in protein prenylation, is a significant cause of statin-induced muscle toxicity. The difference between muscle and liver cell responses is not due to a difference in the protein expression levels of the prenyltransferase enzymes but may perhaps be due to differences in isoprenoid levels, resulting from differences in the protein expression levels of HMGCR between the two. Differences in isoprenoid levels are likely to affect a number of processes, including protein prenylation. In particular, the small GTPase Rap1A showed differential disruption of its prenylation between muscle and liver in this study, which may prove important in the mechanism of statin-induced muscle toxicity. This study highlights the importance of examining the effects of drugs at both the target site of action and at the site of toxicity, rather than only studying one site in isolation, in order to gain insight into the mechanism of adverse drug effects. 


\section{References}

Abela GS, Vedre A, Janoudi A, Huang R, Durga S, Tamhane U (2011) Effect of statins on cholesterol crystallization and atherosclerotic plaque stabilization. Am J Cardiol 107(12):1710-1717

Abrams J (2005) Over-the-counter statins: a new controversy. Nat Clin Pract Cardiovasc Med 2(4):174-175

Aden DP, Fogel A, Plotkin S, Damjanov I, Knowles BB (1979) Controlled synthesis of HBsAg in a differentiated human liver carcinoma-derived cell line. Nature 282(5739):615-616

Aguanno S, Bouche M, Adamo S, Molinaro M (1990) 12-O-tetradecanoylphorbol13-acetate-induced differentiation of a human rhabdomyosarcoma cell line. Cancer Res 50(11):3377-3382

Akashi M, Tsuchiya Y, Yoshino T, Nishida E (2002) Control of intracellular dynamics of mammalian period proteins by casein kinase I epsilon (CKlepsilon) and CKIdelta in cultured cells. Mol Cell Biol 22(6):1693-1703

Akhtar RA, Reddy AB, Maywood ES, Clayton JD, King VM, Smith AG, Gant TW, Hastings MH, Kyriacou CP (2002) Circadian cycling of the mouse liver transcriptome, as revealed by CDNA microarray, is driven by the suprachiasmatic nucleus. Curr Biol 12(7):540-550

Albrecht $U$ (2012) Timing to perfection: the biology of central and peripheral circadian clocks. Neuron 74(2):246-260

Ali BR, Nouvel I, Leung KF, Hume AN, Seabra MC (2010) A novel statinmediated "prenylation block-and-release" assay provides insight into the membrane targeting mechanisms of small GTPases. Biochem Biophys Res Commun 397(1):34-41

Almon RR, Yang E, Lai W, Androulakis IP, Ghimbovschi S, Hoffman EP, Jusko WJ, Dubois DC (2008) Relationships between circadian rhythms and modulation of gene expression by glucocorticoids in skeletal muscle. Am J Physiol Regul Integr Comp Physiol 295(4):R1031-1047

Amaral IP, Johnston IA (2012) Circadian expression of clock and putative clockcontrolled genes in skeletal muscle of the zebrafish. Am J Physiol Regul Integr Comp Physiol 302(1):R193-206

Amundson DM, Zhou M (1999) Fluorometric method for the enzymatic determination of cholesterol. J Biochem Biophys Methods 38(1):43-52 
Andres V, Walsh K (1996) Myogenin expression, cell cycle withdrawal, and phenotypic differentiation are temporally separable events that precede cell fusion upon myogenesis. J Cell Biol 132(4):657-666

Andrews JL, Zhang X, McCarthy JJ, McDearmon EL, Hornberger TA, Russell B, Campbell KS, Arbogast S, Reid MB, Walker JR, Hogenesch JB, Takahashi JS, Esser KA (2010) CLOCK and BMAL1 regulate MyoD and are necessary for maintenance of skeletal muscle phenotype and function. Proc Natl Acad Sci U S A 107(44):19090-19095

Antoine DJ, Srivastava A, Pirmohamed M, Park BK (2010) Statins inhibit aminoglycoside accumulation and cytotoxicity to renal proximal tubule cells. Biochem Pharmacol 79(4):647-654

Applied Biosystems, "TechNotes 8(3): Is Your RNA Intact? Methods to Check RNA Integrity." 2009. (Available from:

http://www.ambion.com/techlib/tn/83/8313.html, Accessed 12th August 2009)

Armstrong SA, Hannah VC, Goldstein JL, Brown MS (1995) CAAX geranylgeranyl transferase transfers farnesyl as efficiently as geranylgeranyl to RhoB. J Biol Chem 270(14):7864-7868

AstraZeneca, "Highlights of Prescribing Information for Crestor (Rosuvastatin calcium)" Package Insert, 2007. (Avaliable from: http://www1.astrazenecaus.com/pi/crestor.pdf, Accessed 28th November 2008)

Baker SK (2005) Molecular clues into the pathogenesis of statin-mediated muscle toxicity. Muscle Nerve 31(5):572-580

Baker SK, Goodwin S, Sur M, Tarnopolsky MA (2004) Cytoskeletal myotoxicity from simvastatin and colchicine. Muscle Nerve 30(6):799-802

Bakhai A, Rigney U, Hollis S, Emmas C (2012) Co-administration of statins with cytochrome P450 3A4 inhibitors in a UK primary care population.

Pharmacoepidemiol Drug Saf(DOI: 10.1002/pds.2308)

Balasubramaniam S, Szanto A, Roach PD (1994) Circadian rhythm in hepatic low-density-lipoprotein (LDL)-receptor expression and plasma LDL levels. Biochem J 298 ( Pt 1):39-43

Balsalobre A, Brown SA, Marcacci L, Tronche F, Kellendonk C, Reichardt HM, Schutz G, Schibler U (2000) Resetting of circadian time in peripheral tissues by glucocorticoid signaling. Science 289(5488):2344-2347

Balsalobre A, Damiola F, Schibler U (1998) A serum shock induces circadian gene expression in mammalian tissue culture cells. Cell 93(6):929-937 
Bandman E (1992) Contractile protein isoforms in muscle development. Dev Biol 154(2):273-283

Bao S, Zhu J, Garvey WT (1998) Cloning of Rab GTPases expressed in human skeletal muscle: studies in insulin-resistant subjects. Horm Metab Res 30(11):656662

Baraldo M (2008) The influence of circadian rhythms on the kinetics of drugs in humans. Expert Opin Drug Metab Toxico/ 4(2):175-192

Barton ER, Park S, James JK, Makarewich CA, Philippou A, Eletto D, Lei H, Brisson B, Ostrovsky O, Li Z, Argon Y (2012) Deletion of muscle GRP94 impairs both muscle and body growth by inhibiting local IGF production. FASEB $J$

Bassel-Duby R, Olson EN (2006) Signaling pathways in skeletal muscle remodeling. Annu Rev Biochem 75:19-37

Beck LA, Hosick TJ, Sinensky M (1990) Isoprenylation is required for the processing of the lamin A precursor. J Cell Biol 110(5):1489-1499

Bellingham C (2001) Cerivastatin withdrawal: the impacts. Pharm J 267(7161):222

Bennett AM, Tonks NK (1997) Regulation of distinct stages of skeletal muscle differentiation by mitogen-activated protein kinases. Science 278(5341):1288-1291

Bifulco M, Laezza C, Aloj SM, Garbi C (1993) Mevalonate controls cytoskeleton organization and cell morphology in thyroid epithelial cells. J Cell Physiol 155(2):340-348

Bjorkhem-Bergman L, Lindh JD, Bergman P (2011) What is a relevant statin concentration in cell experiments claiming pleiotropic effects? Br J Clin Pharmacol 72(1):164-165

Blanco-Colio LM, Villa A, Ortego M, Hernandez-Presa MA, Pascual A, Plaza JJ, Egido J (2002) 3-Hydroxy-3-methyl-glutaryl coenzyme A reductase inhibitors, atorvastatin and simvastatin, induce apoptosis of vascular smooth muscle cells by downregulation of $\mathrm{Bcl}-2$ expression and Rho A prenylation. Atherosclerosis 161(1):17-26

Blasetto JW, Stein EA, Brown WV, Chitra R, Raza A (2003) Efficacy of rosuvastatin compared with other statins at selected starting doses in hypercholesterolemic patients and in special population groups. Am J Cardiol 91(5A):3C-10C

Bolego C, Baetta R, Bellosta S, Corsini A, Paoletti R (2002) Safety considerations for statins. Curr Opin Lipidol 13(6):637-644 
Bos JL (1997) Ras-like GTPases. Biochim Biophys Acta 1333(2):M19-31

Bouche M, Canipari R, Melchionna R, Willems D, Senni MI, Molinaro M (2000) TGF-beta autocrine loop regulates cell growth and myogenic differentiation in human rhabdomyosarcoma cells. FASEB J 14(9):1147-1158

Bozek K, Relogio A, Kielbasa SM, Heine M, Dame C, Kramer A, Herzel H (2009) Regulation of clock-controlled genes in mammals. PLoS One 4(3):e4882

Brockmann R, Beyer A, Heinisch JJ, Wilhelm T (2007) Posttranscriptional expression regulation: what determines translation rates? PLoS Comput Biol 3(3):e57

Brown MS, Goldstein JL (1997) The SREBP pathway: regulation of cholesterol metabolism by proteolysis of a membrane-bound transcription factor. Cell 89(3):331-340

Brown MS, Goldstein JL (2004) Lowering Plasma Cholesterol by Raising LDL Receptors. Atheroscler Supp/ 5(3):57-59

Brugts JJ, Yetgin T, Hoeks SE, Gotto AM, Shepherd J, Westendorp RGJ, de Craen AJM, Knopp RH, Nakamura H, Ridker P, van Domburg R, Deckers JW (2009) The benefits of statins in people without established cardiovascular disease but with cardiovascular risk factors: meta-analysis of randomised controlled trials. BMJ 339(338:b2376. doi: 10.1136/bmj.b2376.)

Bunger MK, Wilsbacher LD, Moran SM, Clendenin C, Radcliffe LA, Hogenesch JB, Simon MC, Takahashi JS, Bradfield CA (2000) Mop3 is an essential component of the master circadian pacemaker in mammals. Cell 103(7):1009-1017

Busino L, Bassermann F, Maiolica A, Lee C, Nolan PM, Godinho SIH, Draetta GF, Pagano M (2007) SCFFbxl3 Controls the Oscillation of the Circadian Clock by Directing the Degradation of Cryptochrome Proteins. Science 316(5826):900-904

Caldwell J, Gardner I, Swales N (1995) An introduction to drug disposition: the basic principles of absorption, distribution, metabolism, and excretion. Toxicol Pathol 23(2):102-114

Cao P, Hanai J-i, Tanksale P, Imamura S, Sukhatme VP, Lecker SH (2009) Statin-induced muscle damage and atrogin-1 induction is the result of a geranylgeranylation defect. FASEB J 23(9):2844-2854

Carey KA, Segal D, Klein R, Sanigorski A, Walder K, Collier GR, Cameron-Smith D (2006) Identification of novel genes expressed during rhabdomyosarcoma differentiation using cDNA microarrays. Pathol Int 56(5):246-255 
Carlotti F, Bazuine M, Kekarainen T, Seppen J, Pognonec P, Maassen JA, Hoeben RC (2004) Lentiviral vectors efficiently transduce quiescent mature 3T3-L1 adipocytes. Mol Ther 9(2):209-217

Casey PJ, Seabra MC (1996) Protein prenyltransferases. J Biol Chem 271(10):5289-5292

Casey PJ, Solski PA, Der CJ, Buss JE (1989) p21ras is modified by a farnesyl isoprenoid. Proc Natl Acad Sci USA 86(21):8323-8327

Castro AF, Campos T, Babcock JT, Armijo ME, Martinez-Conde A, Pincheira R, Quilliam LA (2011) M-Ras induces Ral and JNK activation to regulate MEK/ERKindependent gene expression in MCF-7 breast cancer cells. Journal of cellular biochemistry(doi: 10.1002/jcb.23458)

Castro AF, Rebhun JF, Quilliam LA (2005) Measuring Ras-family GTP levels in vivo--running hot and cold. Methods 37(2):190-196

Catapano AL (2007) The pharmacologic elegance of inhibiting cholesterol absorption and synthesis while providing a homeostatic balance. Fundam Clin Pharmacol 21 (Suppl 2):21-26

Chalmers JA, Martino TA, Tata N, Ralph MR, Sole MJ, Belsham DD (2008) Vascular circadian rhythms in a mouse vascular smooth muscle cell line (Movas1). Am J Physiol Regul Integr Comp Physiol 295(5):R1529-R1538

Chan KKW, Oza AM, Siu LL (2003) The statins as anticancer agents. Clin Cancer $\operatorname{Res}$ 9(1):10-19

Chapman MJ (2010) Cardiovascular diseases. Introduction. Atheroscler Suppl 11(3):1-2

Charlton-Menys V, Durrington PN (2008) Human cholesterol metabolism and therapeutic molecules. Exp Physio/ 93(1):27-42

Chen M, Feng HZ, Gupta D, Kelleher J, Dickerson KE, Wang J, Hunt D, Jou W, Gavrilova O, Jin JP, Weinstein LS (2009a) G(s)alpha deficiency in skeletal muscle leads to reduced muscle mass, fiber-type switching, and glucose intolerance without insulin resistance or deficiency. Am J Physiol Cell Physiol 296(4):C930-940

Chen Y, Coulter S, Jetten AM, Goldstein JA (2009b) Identification of human CYP2C8 as a retinoid-related orphan nuclear receptor target gene. J Pharmacol Exp Ther 329(1):192-201

Chiba Y, Sato S, Misawa M (2010) Upregulation of geranylgeranyltransferase I in bronchial smooth muscle of mouse experimental asthma: its inhibition by lovastatin. J Smooth Muscle Res 46(1):57-64 
Chong PH, Seeger JD, Franklin C (2001) Clinically relevant differences between the statins: implications for therapeutic selection. Am J Med 111(5):390-400

Cohn RD, Henry MD, Michele DE, Barresi R, Saito F, Moore SA, Flanagan JD, Skwarchuk MW, Robbins ME, Mendell JR, Williamson RA, Campbell KP (2002) Disruption of DAG1 in differentiated skeletal muscle reveals a role for dystroglycan in muscle regeneration. Cell 110(5):639-648

Cordle A, Koenigsknecht-Talboo J, Wilkinson B, Limpert A, Landreth G (2005) Mechanisms of statin-mediated inhibition of small G-protein function. J Biol Chem 280(40):34202-34209

Coxon FP, Ebetino FH, Mules EH, Seabra MC, McKenna CE, Rogers MJ (2005) Phosphonocarboxylate inhibitors of Rab geranylgeranyl transferase disrupt the prenylation and membrane localization of Rab proteins in osteoclasts in vitro and in vivo. Bone 37(3):349-358

Crumbley C, Burris TP (2011) Direct Regulation of CLOCK Expression by REVERB. PLoS One 6(3):e17290

Crumbley C, Wang Y, Kojetin DJ, Burris TP (2010) Characterization of the core mammalian clock component, NPAS2, as a REV-ERBalpha/RORalpha target gene. J Biol Chem 285(46):35386-35392

Csibi A, Tintignac LA, Leibovitch MP, Leibovitch SA (2008) elF3-f function in skeletal muscles: to stand at the crossroads of atrophy and hypertrophy. Cell Cycle 7(12):1698-1701

da Costa N, Edgar J, Ooi PT, Su Y, Meissner JD, Chang KC (2007) Calcineurin differentially regulates fast myosin heavy chain genes in oxidative muscle fibre type conversion. Cell Tissue Res 329(3):515-527

Damiola F, Le Minh N, Preitner N, Kornmann B, Fleury-Olela F, Schibler U (2000) Restricted feeding uncouples circadian oscillators in peripheral tissues from the central pacemaker in the suprachiasmatic nucleus. Genes Dev 14:2950 - 2961

Davies BS, Fong LG, Yang SH, Coffinier C, Young SG (2009) The posttranslational processing of prelamin A and disease. Annu Rev Genomics Hum Genet 10:153-174

DeBruyne JP, Noton E, Lambert CM, Maywood ES, Weaver DR, Reppert SM (2006) A clock shock: mouse CLOCK is not required for circadian oscillator function. Neuron 50(3):465-477

DeBruyne JP, Weaver DR, Reppert SM (2007) CLOCK and NPAS2 have overlapping roles in the suprachiasmatic circadian clock. Nat Neurosci 10(5):543545 
Dibner C, Schibler U, Albrecht U (2010) The mammalian circadian timing system: organization and coordination of central and peripheral clocks. Annu Rev Physiol 72:517-549

Dirks AJ, Jones KM (2006) Statin-induced apoptosis and skeletal myopathy Am J Physiol Cell Physiol, p C1208-1212

Ehrhardt GR, Leslie KB, Lee F, Wieler JS, Schrader JW (1999) M-Ras, a widely expressed 29-kD homologue of p21 Ras: expression of a constitutively active mutant results in factor-independent growth of an interleukin-3-dependent cell line. Blood 94(7):2433-2444

Eide EJ, Vielhaber EL, Hinz WA, Virshup DM (2002) The circadian regulatory proteins BMAL1 and cryptochromes are substrates of casein kinase I epsilon. $J$ Biol Chem 277(19):17248-17254

Elsayed RK, Evans JD (2008) Emerging lipid-lowering drugs: squalene synthase inhibitors. Expert Opin Emerg Drugs 13(2):309-322

Farnsworth CC, Wolda SL, Gelb MH, Glomset JA (1989) Human lamin B contains a farnesylated cysteine residue. J Biol Chem 264(34):20422-20429

Favre B, Schneider Y, Lingasamy P, Bouameur JE, Begre N, Gontier Y, SteinerChampliaud MF, Frias MA, Borradori L, Fontao L (2011) Plectin interacts with the rod domain of type III intermediate filament proteins desmin and vimentin. Eur $J$ Cell Biol 90(5):390-400

Festing MF, Altman DG (2002) Guidelines for the design and statistical analysis of experiments using laboratory animals. ILAR J 43(4):244-258

Flint OP, Masters BA, Gregg RE, Durham SK (1997) Inhibition of Cholesterol Synthesis by Squalene Synthase Inhibitors Does Not Induce Myotoxicity in Vitro. Toxicol Appl Pharmacol 145(1):91-98

Folch J, Lees M, Stanley GHS (1957) A Simple Method for the Isolation and Purification of Total Lipides from Animal Tissues. J Biol Chem 226(1):497-509

Friday BB, Horsley V, Pavlath GK (2000) Calcineurin activity is required for the initiation of skeletal muscle differentiation. J Cell Biol 149(3):657-666

Frische EW, Zwartkruis FJ (2010) Rap1, a mercenary among the Ras-like GTPases. Dev Biol 340(1):1-9

Fukata M, Nakagawa M, Kaibuchi K (2003) Roles of Rho-family GTPases in cell polarisation and directional migration. Curr Opin Cell Biol 15(5):590-597

Gachon F, Firsov D (2011) The role of the circadian timing system on drug metabolism and detoxification. Expert Opin Drug Metab Toxicol 7(2):147-158 
Gälman C, Angelin B, Rudling M (2005) Bile Acid Synthesis in Humans Has a Rapid Diurnal Variation That Is Asynchronous With Cholesterol Synthesis. Gastroenterology 129(5):1445-1453

Gamble W, Vaughan M, Kruth HS, Avigan J (1978) Procedure for determination of free and total cholesterol in micro- or nanogram amounts suitable for studies with cultured cells. J Lipid Res 19(8):1068-1070

Godinho SIH, Maywood ES, Shaw L, Tucci V, Barnard AR, Busino L, Pagano M, Kendall R, Quwailid MM, Romero MR, O'Neill J, Chesham JE, Brooker D, Lalanne Z, Hastings MH, Nolan PM (2007) The After-Hours Mutant Reveals a Role for Fbxl3 in Determining Mammalian Circadian Period. Science 316(5826):897-900

Goldstein JL, Brown MS (1990) Regulation of the mevalonate pathway. Nature 343(6257):425-430

Gomes MD, Lecker SH, Jagoe RT, Navon A, Goldberg AL (2001) Atrogin-1, a muscle-specific F-box protein highly expressed during muscle atrophy. Proc NatI Acad Sci USA 98(25):14440-14445

Gotto AM, Jr., Moon JE (2012) Recent clinical studies of the effects of lipidmodifying therapies. Am J Cardiol 110(1 Suppl):15A-26A

Greenbaum D, Colangelo C, Williams K, Gerstein M (2003) Comparing protein abundance and mRNA expression levels on a genomic scale. Genome Biol 4(9):117

Griffin TJ, Gygi SP, Ideker T, Rist B, Eng J, Hood L, Aebersold R (2002) Complementary profiling of gene expression at the transcriptome and proteome levels in Saccharomyces cerevisiae. Mol Cell Proteomics 1(4):323-333

Gullestad L, Oie E, Ueland T, Yndestad A, Aukrust P (2007) The role of statins in heart failure. Fundam Clin Pharmacol 21 Suppl 2:35-40

Guo CS, Degnin C, Fiddler TA, Stauffer D, Thayer MJ (2003) Regulation of MyoD activity and muscle cell differentiation by MDM2, pRb, and Sp1. J Biol Chem 278(25):22615-22622

Guo X, Schrader KA, Xu Y, Schrader JW (2005) Expression of a constitutively active mutant of $M-$ Ras in normal bone marrow is sufficient for induction of a malignant mastocytosis/mast cell leukemia, distinct from the histiocytosis/monocytic leukemia induced by expression of activated $\mathrm{H}$-Ras.

Oncogene 24(14):2330-2342

Hamelin BA, Turgeon J (1998) Hydrophilicity/ lipophilicity: relevance for the pharmacology and clinical effects of HMG-CoA reductase inhibitors. Trends Pharmacol Sci 19(1):26-37 
Harper CR, Jacobson TA (2007) The broad spectrum of statin myopathy: from myalgia to rhabdomyolysis. Curr Opin Lipidol 18(4):401-408

Harper CR, Jacobson TA (2010) Evidence-based management of statin myopathy. Curr Atheroscler Rep 12(5):322-330

Heasman SJ, Ridley AJ (2008) Mammalian Rho GTPases: new insights into their functions from in vivo studies. Nat Rev Mol Cell Biol 9(9):690-701

Hedenmalm K, Alvan G, Ohagen P, Dahl ML (2010) Muscle toxicity with statins. Pharmacoepidemiol Drug Saf 19(3):223-231

Hers I, Vincent EE, Tavare JM (2011) Akt signalling in health and disease. Cell Signal 23(10):1515-1527

Hilgendorf C, Ahlin G, Seithel A, Artursson P, Ungell AL, Karlsson J (2007) Expression of thirty-six drug transporter genes in human intestine, liver, kidney, and organotypic cell lines. Drug Metab Dispos 35(8):1333-1340

Hirota T, Fukada Y (2004) Resetting mechanism of central and peripheral circadian clocks in mammals. Zoolog Sci 21(4):359-368

Hlatky MA (2008) Expanding the Orbit of Primary Prevention -- Moving beyond JUPITER. N Engl J Med:NEJMe0808320

Honma S, Kawamoto T, Takagi Y, Fujimoto K, Sato F, Noshiro M, Kato Y, Honma $\mathrm{K}$ (2002) Dec1 and Dec2 are regulators of the mammalian molecular clock. Nature 419(6909):841-844

Hsiang B, Zhu Y, Wang Z, Wu Y, Sasseville V, Yang W-P, Kirchgessner TG (1999) A Novel Human Hepatic Organic Anion Transporting Polypeptide (OATP2). Identification of a Liver-Specific Human Organic Anion Transporting Polypeptide and Identification of Rat and Human Hydroxymethylglutaryl-CoA Reductase Inhibitor Transporters. J Biol Chem 274(52):37161-37168

Huang TS, Grodeland G, Sleire L, Wang MY, Kvalheim G, Laerum OD (2009) Induction of circadian rhythm in cultured human mesenchymal stem cells by serum shock and cAMP analogs in vitro. Chronobiol Int 26(2):242-257

Innominato PF, Levi FA, Bjarnason GA (2010) Chronotherapy and the molecular clock: Clinical implications in oncology. Adv Drug Deliv Rev 62(9-10):979-1001

Isis Innovation, "Isis licensee launches test for statin side effects" Press Release, 2012. (Avaliable from: http://www.isisinnovation.com/news/news//sisLicenseeLaunchesTestforStatinSideEffects.html, Accessed 3rd August 2012) 
Istvan ES, Deisenhofer J (2001) Structural Mechanism for Statin Inhibition of HMG-CoA Reductase. Science 292(5519):1160-1164

Itagaki M, Takaguri A, Kano S, Kaneta S, Ichihara K, Satoh K (2009) Possible mechanisms underlying statin-induced skeletal muscle toxicity in L6 fibroblasts and in rats. J Pharmacol Sci 109(1):94-101

Jacobson J (2008) Statins in Endothelial Signaling and Activation. Antioxidants \& Redox Signaling $\mathbf{0}(\mathrm{ja})$

Jick H, Wilson A, Wiggins P, Chamberlin DP (2012) Comparison of prescription drug costs in the United States and the United kingdom, part 1: statins.

Pharmacotherapy 32(1):1-6

Jin M, Saucan L, Farquhar MG, Palade GE (1996) Rab1a and multiple other Rab proteins are associated with the transcytotic pathway in rat liver. $J$ Biol Chem 271(47):30105-30113

Joint Formulary Committee; British National Formulary, 56th Edition. 2008, London: British Medical Association and Royal Pharmaceutical Society of Great Britain

Jones PJ, Schoeller DA (1990) Evidence for diurnal periodicity in human cholesterol synthesis. J Lipid Res 31(4):667-673

Jurynec MJ, Xia R, Mackrill JJ, Gunther D, Crawford T, Flanigan KM, Abramson JJ, Howard MT, Grunwald DJ (2008) Selenoprotein N is required for ryanodine receptor calcium release channel activity in human and zebrafish muscle. Proc Natl Acad Sci USA 105(34):12485-12490

Kamphuis W, Cailotto C, Dijk F, Bergen A, Buijs RM (2005) Circadian expression of clock genes and clock-controlled genes in the rat retina. Biochem Biophys Res Commun 330(1):18-26

Kang HS, Angers M, Beak JY, Wu X, Gimble JM, Wada T, Xie W, Collins JB, Grissom SF, Jetten AM (2007) Gene expression profiling reveals a regulatory role for ROR alpha and ROR gamma in phase I and phase II metabolism. Physiol Genomics 31(2):281-294

Keduka E, Kaiho A, Hamada M, Watanabe-Takano H, Takano K, Ogasawara M, Satou Y, Satoh N, Endo T (2009) M-Ras evolved independently of R-Ras and its neural function is conserved between mammalian and ascidian, which lacks classical Ras. Gene 429(1-2):49-58

Khan N, Shen J, Chang TY, Chang CC, Fung PC, Grinberg O, Demidenko E, Swartz H (2003) Plasma membrane cholesterol: a possible barrier to intracellular oxygen in normal and mutant $\mathrm{CHO}$ cells defective in cholesterol metabolism.

Biochemistry 42(1):23-29 
Khan OM, Ibrahim MX, Jonsson IM, Karlsson C, Liu M, Sjogren AK, Olofsson FJ, Brisslert M, Andersson S, Ohlsson C, Hulten LM, Bokarewa M, Bergo MO (2011) Geranylgeranyltransferase type I (GGTase-I) deficiency hyperactivates macrophages and induces erosive arthritis in mice. J Clin Invest 121(2):628-639

Kimmelman A, Tolkacheva T, Lorenzi MV, Osada M, Chan AM (1997) Identification and characterization of R-ras3: a novel member of the RAS gene family with a non-ubiquitous pattern of tissue distribution. Oncogene 15(22):26752685

Kitamura S, Maeda K, Wang Y, Sugiyama Y (2008) Involvement of Multiple Transporters in the Hepatobiliary Transport of Rosuvastatin. Drug Metab Dispos 36(10):2014-2023

Knauer MJ, Urquhart BL, Meyer zu Schwabedissen HE, Schwarz UI, Lemke CJ, Leake BF, Kim RB, Tirona RG (2010) Human skeletal muscle drug transporters determine local exposure and toxicity of statins. Circ Res 106(2):297-306

Knowles BB, Howe CC, Aden DP (1980) Human hepatocellular carcinoma cell lines secrete the major plasma proteins and hepatitis B surface antigen. Science 209(4455):497-499

Knudsen ES, Pazzagli C, Born TL, Bertolaet BL, Knudsen KE, Arden KC, Henry RR, Feramisco JR (1998) Elevated cyclins and cyclin-dependent kinase activity in the rhabdomyosarcoma cell line RD. Cancer Res 58(9):2042-2049

$\mathrm{Ko} \mathrm{CH}$, Takahashi JS (2006) Molecular components of the mammalian circadian clock. Hum Mol Genet 15(suppl 2):R271-277

Kobayashi M, Chisaki I, Narumi K, Hidaka K, Kagawa T, Itagaki S, Hirano T, Iseki K (2008) Association between risk of myopathy and cholesterol-lowering effect: A comparison of all statins. Life Sci 82(17-18):969-975

Kohsaka A, Bass J (2007) A sense of time: how molecular clocks organize metabolism. Trends Endocrinol Metab 18(1):4-11

Kowa Pharmaceutical Europe, "Livazo® (pitavastatin), a new potent statin with beneficial effects on lipid fractions associated with heart disease, launches in Spain" Press Release, 2011. (Available from:

http://www.kowapharmaceuticals.eu/media/2771/003 spanish launch 280411.pdf, Accessed 24th April 2012)

Koyanagi S, Okazawa S, Kuramoto Y, Ushijima K, Shimeno H, Soeda S, Okamura $\mathrm{H}$, Ohdo S (2006) Chronic treatment with prednisolone represses the circadian oscillation of clock gene expression in mouse peripheral tissues. Mol Endocrinol 20(3):573-583 
Kromer A, Moosmann B (2009) Statin-Induced Liver Injury Involves Cross-Talk between Cholesterol and Selenoprotein Biosynthetic Pathways. Mol Pharmacol 75(6):1421-1429

Kuipers HF, Rappert AA, Mommaas AM, van Haastert ES, van der Valk $P$, Boddeke HW, Biber KP, van den Elsen PJ (2006) Simvastatin affects cell motility and actin cytoskeleton distribution of microglia. Glia 53(2):115-123

Lackner MR, Kindt RM, Carroll PM, Brown K, Cancilla MR, Chen C, de Silva $\mathrm{H}$, Franke Y, Guan B, Heuer T, Hung T, Keegan K, Lee JM, Manne V, O'Brien C, Parry D, Perez-Villar JJ, Reddy RK, Xiao H, Zhan H, Cockett M, Plowman G, Fitzgerald K, Costa M, Ross-Macdonald P (2005) Chemical genetics identifies Rab geranylgeranyl transferase as an apoptotic target of farnesyl transferase inhibitors. Cancer cell 7(4):325-336

Lamia KA, Sachdeva UM, DiTacchio L, Williams EC, Alvarez JG, Egan DF, Vasquez DS, Juguilon H, Panda S, Shaw RJ, Thompson CB, Evans RM (2009) AMPK regulates the circadian clock by cryptochrome phosphorylation and degradation. Science 326(5951):437-440

Lammerding J, Schulze PC, Takahashi T, Kozlov S, Sullivan T, Kamm RD, Stewart CL, Lee RT (2004) Lamin A/C deficiency causes defective nuclear mechanics and mechanotransduction. J Clin Invest 113(3):370-378

Lane KT, Beese LS (2006) Thematic review series: lipid posttranslational modifications. Structural biology of protein farnesyltransferase and geranylgeranyltransferase type I. J Lipid Res 47(4):681-699

Law M, Rudnicka AR (2006) Statin safety: a systematic review. Am J Cardiol 97(8A):52C-60C

Le Martelot G, Claudel T, Gatfield D, Schaad O, Kornmann B, Sasso GL, Moschetta A, Schibler U (2009) REV-ERBalpha participates in circadian SREBP signaling and bile acid homeostasis. PLOS Biol 7(9):e1000181

Lee R, Chang SY, Trinh H, Tu Y, White AC, Davies BS, Bergo MO, Fong LG, Lowry WE, Young SG (2010) Genetic studies on the functional relevance of the protein prenyltransferases in skin keratinocytes. Hum Mol Genet 19(8):1603-1617

Leibetseder V, Humpeler S, Svoboda M, Schmid D, Thalhammer T, Zuckermann A, Marktl W, Ekmekcioglu C (2009) Clock genes display rhythmic expression in human hearts. Chronobiol Int 26(4):621-636

Lemos DR, Goodspeed L, Tonelli L, Antoch MP, Ojeda SR, Urbanski HF (2007) Evidence for circadian regulation of activating transcription factor 5 but not tyrosine hydroxylase by the chromaffin cell clock. Endocrinology 148(12):5811-5821 
Lerner EC, Qian Y, Hamilton AD, Sebti SM (1995) Disruption of oncogenic KRas4B processing and signaling by a potent geranylgeranyltransferase I inhibitor. $J$ Biol Chem 270(45):26770-26773

Leung KF, Baron R, Seabra MC (2006) Thematic review series: lipid posttranslational modifications. geranylgeranylation of Rab GTPases. J Lipid Res 47(3):467-475

Levi F, Schibler U (2007) Circadian rhythms: mechanisms and therapeutic implications. Annu Rev Pharmacol Toxicol 47:593-628

Li R, Yue J, Zhang Y, Zhou L, Hao W, Yuan J, Qiang B, Ding JM, Peng X, Cao JM (2008) CLOCK/BMAL1 regulates human nocturnin transcription through binding to the E-box of nocturnin promoter. Mol Cell Biochem 317(1-2):169-177

Liang SL, Liu H, Zhou A (2006) Lovastatin-induced apoptosis in macrophages through the Rac1/Cdc42/JNK pathway. J Immunol 177(1):651-656

Liao JK (2002) Isoprenoids as mediators of the biological effects of statins. J Clin Invest 110(3):285-288

Liao JK, Laufs U (2005) Pleiotropic Effects of Statins. Annu Rev Pharmacol Toxicol 45(1):89-118

Link E, Parish S, Armitage J, Bowman L, Heath S, Matsuda F, Gut I, Lathrop M, Collins R (2008) SLCO1B1 variants and statin-induced myopathy--a genomewide study. N Engl J Med 359(8):789-799

Lluis F, Perdiguero E, Nebreda AR, Munoz-Canoves P (2006) Regulation of skeletal muscle gene expression by p38 MAP kinases. Trends Cell Biol 16(1):3644

Lokireddy S, Wijesoma IW, Sze SK, McFarlane C, Kambadur R, Sharma M (2012) Identification of Atrogin-1 targeted proteins during the Myostatin-induced skeletal muscle wasting. Am J Physiol Cell Physiol

Lowrey PL, Shimomura K, Antoch MP, Yamazaki S, Zemenides PD, Ralph MR, Menaker M, Takahashi JS (2000) Positional syntenic cloning and functional characterization of the mammalian circadian mutation tau. Science 288(5465):483492

Lowry OH, Rosebrough NJ, Farr AL, Randall RJ (1951) Protein measurement with the Folin phenol reagent. J Biol Chem 193(1):265-275

Lutz RJ, Trujillo MA, Denham KS, Wenger L, Sinensky M (1992) Nucleoplasmic Localization of Prelamin-a - Implications for Prenylation-Dependent Lamin-a Assembly into the Nuclear Lamina. Proc Natl Acad Sci USA 89(7):3000-3004 
Ma H, Urquidi V, Wong J, Kleeman J, Goodison S (2003) Telomerase reverse transcriptase promoter regulation during myogenic differentiation of human RD rhabdomyosarcoma cells. Mol Cancer Res 1(10):739-746

Mackall JC, Student AK, Polakis SE, Lane MD (1976) Induction of lipogenesis during differentiation in a "preadipocyte" cell line. J Biol Chem 251(20):6462-6464

Madsen CS, Janovitz E, Zhang R, Nguyen-Tran V, Ryan CS, Yin X, Monshizadegan H, Chang M, D'Arienzo C, Scheer S, Setters R, Search D, Chen X, Zhuang S, Kunselman L, Peters A, Harrity T, Apedo A, Huang C, Cuff CA, Kowala MC, Blanar MA, Sun CQ, Robl JA, Stein PD (2008) The Guinea pig as a preclinical model for demonstrating the efficacy and safety of statins. $J$ Pharmacol Exp Ther 324(2):576-586

Makabe S, Takahashi Y, Watanabe H, Murakami M, Ohba T, Ito H (2010) Fluvastatin protects vascular smooth muscle cells against oxidative stress through the Nrf2-dependent antioxidant pathway. Atherosclerosis 213(2):377-384

Marcoff L, Thompson PD (2007) The role of coenzyme Q10 in statin-associated myopathy: a systematic review. J Am Coll Cardiol 49(23):2231-2237

Maronde E, Motzkus D (2003) Oscillation of human period 1 (hPER1) reporter gene activity in human neuroblastoma cells in vivo. Chronobiol Int 20(4):671-681

Martin-Rendon E, Blake DJ (2003) Protein glycosylation in disease: new insights into the congenital muscular dystrophies. Trends Pharmacol Sci 24(4):178-183

Martin AM, Elliott JA, Duffy P, Blake CM, Ben Attia S, Katz LM, Browne JA, Gath V, McGivney BA, Hill EW, Murphy BA (2010) Circadian regulation of locomotor activity and skeletal muscle gene expression in the horse. J Appl Physiol

109(5):1328-1336

Martin PD, Mitchel PD, Schneck DW (2002) Pharmacodynamic effects and pharmacokinetics of a new HMG-CoA reductase inhibitor, rosuvastatin, after morning or evening administration in healthy volunteers. Br J Clin Pharmacol 54(5):472-477

Martini C, Trapani L, Narciso L, Marino M, Trentalance A, Pallottini V (2009) 3hydroxy 3-methylglutaryl coenzyme A reductase increase is essential for rat muscle differentiation. J Cell Physiol 220(2):524-530

Mason RP (2006) Molecular basis of differences among statins and a comparison with antioxidant vitamins. Am J Cardiol 98(11):34P-41P

Mastaglia FL, Needham M (2012) Update on toxic myopathies. Curr Neurol Neurosci Rep 12(1):54-61 
Matozaki T, Nakanishi H, Takai Y (2000) Small G-protein networks: Their crosstalk and signal cascades. Cell Signal 12(8):515-524

Matsumoto K, Asano T, Endo T (1997) Novel small GTPase M-Ras participates in reorganization of actin cytoskeleton. Oncogene 15(20):2409-2417

Matsunaga N, Ikeda M, Takiguchi T, Koyanagi S, Ohdo S (2008) The molecular mechanism regulating 24-hour rhythm of CYP2E1 expression in the mouse liver. Hepatology 48(1):240-251

Matzno S, Yamauchi T, Gohda M, Ishida N, Katsuura K, Hanasaki Y, Tokunaga T, Itoh H, Nakamura N (1997) Inhibition of cholesterol biosynthesis by squalene epoxidase inhibitor avoids apoptotic cell death in L6 myoblasts. J Lipid Res 38(8):1639-1648

Matzno S, Yasuda S, Juman S, Yamamoto Y, Nagareya-Ishida N, TazuyaMurayama K, Nakabayashi T, Matsuyama K (2005) Statin-induced apoptosis linked with membrane farnesylated Ras small $G$ protein depletion, rather than geranylated Rho protein. J Pharm Pharmacol 57(11):1475-1484

Maurer-Stroh S, Washietl S, Eisenhaber F (2003) Protein prenyltransferases. Genome Biol 4(4):212

McAdams M, Staffa J, Dal Pan G (2008) Estimating the extent of reporting to FDA: a case study of statin-associated rhabdomyolysis. Pharmacoepidemiol Drug Saf 17(3):229-239

McAllister RM, Melnyk J, Finkelstein JZ, Adams EC, Jr., Gardner MB (1969) Cultivation in vitro of cells derived from a human rhabdomyosarcoma. Cancer 24(3):520-526

McCarthy JJ, Andrews JL, McDearmon EL, Campbell KS, Barber BK, Miller BH, Walker JR, Hogenesch JB, Takahashi JS, Esser KA (2007) Identification of the circadian transcriptome in adult mouse skeletal muscle. Physiol Genomics 31(1):86-95

McTaggart SJ (2006) Isoprenylated proteins. Cell Mol Life Sci 63(3):255-267

Megeney LA, Rudnicki MA (1995) Determination versus differentiation and the MyoD family of transcription factors. Biochem Cell Biol 73(9-10):723-732

Meng QJ, Logunova L, Maywood ES, Gallego M, Lebiecki J, Brown TM, Sladek M, Semikhodskii AS, Glossop NR, Piggins HD, Chesham JE, Bechtold DA, Yoo SH, Takahashi JS, Virshup DM, Boot-Handford RP, Hastings MH, Loudon AS (2008) Setting clock speed in mammals: the CK1 epsilon tau mutation in mice accelerates circadian pacemakers by selectively destabilizing PERIOD proteins. Neuron 58(1):78-88 
Mensink RP, Aro A, Den Hond E, German JB, Griffin BA, ten Meer HU, Mutanen M, Pannemans D, Stahl W (2003) PASSCLAIM - Diet-related cardiovascular disease. Eur J Nutr 42 Suppl 1:16-27

Meske V, Albert F, Richter D, Schwarze J, Ohm TG (2003) Blockade of HMGCoA reductase activity causes changes in microtubule-stabilizing protein tau via suppression of geranylgeranylpyrophosphate formation: implications for Alzheimer's disease. Eur J Neurosci 17(1):93-102

Mihaylova B, Emberson J, Blackwell L, Keech A, Simes J, Barnes EH, Voysey M, Gray A, Collins R, Baigent C (2012) The effects of lowering LDL cholesterol with statin therapy in people at low risk of vascular disease: meta-analysis of individual data from 27 randomised trials. Lancet:DOI:10.1016/S0140-6736(1012)6036760365

Mitsui S, Yamaguchi S, Matsuo T, Ishida Y, Okamura H (2001) Antagonistic role of E4BP4 and PAR proteins in the circadian oscillatory mechanism. Genes Dev 15(8):995-1006

Miyazaki M, Schroder E, Edelmann SE, Hughes ME, Kornacker K, Balke CW, Esser KA (2011) Age-associated disruption of molecular clock expression in skeletal muscle of the spontaneously hypertensive rat. PLoS One 6(11):e27168

Mohaupt MG, Karas RH, Babiychuk EB, Sanchez-Freire V, Monastyrskaya K, lyer L, Hoppeler H, Breil F, Draeger A (2009) Association between statin-associated myopathy and skeletal muscle damage. CMAJ 181(1-2):E11-18

Moosmann B, Behl C (2004) Selenoprotein synthesis and side-effects of statins. Lancet 363(9412):892-894

Moran JL, Li Y, Hill AA, Mounts WM, Miller CP (2002) Gene expression changes during mouse skeletal myoblast differentiation revealed by transcriptional profiling. Physiol Genomics 10(2):103-111

Mosmann T (1983) Rapid colorimetric assay for cellular growth and survival: application to proliferation and cytotoxicity assays. J Immunol Methods 65(1-2):5563

Muck W, Frey R, Unger S, Voith B (2000) Pharmacokinetics of cerivastatin when administered under fasted and fed conditions in the morning or evening. Int $J$ Clin Pharmacol Ther 38(6):298-303

Mukhtar RY, Reid J, Reckless JP (2005) Pitavastatin. Int J Clin Pract 59(2):239252

Mullen PJ, Lüscher B, Scharnagl H, Krähenbühl S, Brecht K (2010) Effect of simvastatin on cholesterol metabolism in $\mathrm{C} 2 \mathrm{C} 12$ myotubes and HepG2 cells, and consequences for statin-induced myopathy. Biochem Pharmacol 79(8):1200-1209 
Mullen PJ, Zahno A, Lindinger P, Maseneni S, Felser A, Krahenbuhl S, Brecht K (2011) Susceptibility to simvastatin-induced toxicity is partly determined by mitochondrial respiration and phosphorylation state of Akt. Biochim Biophys Acta 1813(12):2079-2087

Muntoni F, Brockington M, Torelli S, Brown SC (2004) Defective glycosylation in congenital muscular dystrophies. Curr Opin Neurol 17(2):205-209

Muttenthaler M, Alewood PF (2008) Selenopeptide chemistry. J Pept Sci 14(12):1223-1239

Nagoshi E, Saini C, Bauer C, Laroche T, Naef F, Schibler U (2004) Circadian gene expression in individual fibroblasts: cell-autonomous and self-sustained oscillators pass time to daughter cells. Cell 119(5):693-705

Nakabayashi H, Taketa K, Miyano K, Yamane T, Sato J (1982) Growth of human hepatoma cells lines with differentiated functions in chemically defined medium.

Cancer Res 42(9):3858-3863

NanoDrop, "Technical Support Bulletin T009: 260/280 and 260/230 Ratios" 2007. (Available from:

http://www.biolabanalytical.com.au/analytical/lifescience/nanodrop/pdf/T009-ND1000-\&-ND-8000-Nucleic-Acid-Purity-Ratios.pdf, Accessed 12th August 2009)

National Institute for Health and Clinical Excellence, "Statins for the Prevention of Cardiovascular Events (Technology Appraisal 94)" 2006. (Avaliable from: http://www.nice.org.uk/TA094, Accessed 14th June 2011)

Nishimoto T, Ishikawa E, Anayama H, Hamajyo H, Nagai H, Hirakata M, Tozawa R (2007) Protective effects of a squalene synthase inhibitor, lapaquistat acetate (TAK-475), on statin-induced myotoxicity in guinea pigs. Toxicol Appl Pharmacol 223(1):39-45

Nishimoto T, Tozawa R, Amano Y, Wada T, Imura Y, Sugiyama Y (2003) Comparing myotoxic effects of squalene synthase inhibitor, T-91485, and 3hydroxy-3-methylglutaryl coenzyme A (HMG-CoA) reductase inhibitors in human myocytes. Biochem Pharmacol 66(11):2133-2139

Noshiro M, Kawamoto T, Furukawa M, Fujimoto K, Yoshida Y, Sasabe E, Tsutsumi S, Hamada T, Honma S, Honma K, Kato Y (2004) Rhythmic expression of DEC1 and DEC2 in peripheral tissues: DEC2 is a potent suppressor for hepatic cytochrome P450s opposing DBP. Genes Cells 9(4):317-329

Noshiro M, Usui E, Kawamoto T, Kubo H, Fujimoto K, Furukawa M, Honma S, Makishima M, Honma K, Kato Y (2007) Multiple mechanisms regulate circadian expression of the gene for cholesterol 7alpha-hydroxylase (Cyp7a), a key enzyme in hepatic bile acid biosynthesis. J Biol Rhythms 22(4):299-311 
Nuoffer C, Davidson HW, Matteson J, Meinkoth J, Balch WE (1994) A GDPbound form of rab1 inhibits protein export from the endoplasmic reticulum and transport between Golgi compartments. J Cell Biol 125(2):225-237

Oh J, Ban MR, Miskie BA, Pollex RL, Hegele RA (2007) Genetic determinants of statin intolerance. Lipids Health Dis 6:7

Ohdo S (2010) Chronotherapeutic strategy: Rhythm monitoring, manipulation and disruption. Adv Drug Deliv Rev 62(9-10):859-875

Ohsaki K, Oishi K, Kozono Y, Nakayama K, Nakayama KI, Ishida N (2008) The role of $\{$ beta\}-TrCP1 and \{beta\}-TrCP2 in circadian rhythm generation by mediating degradation of clock protein PER2. J Biochem 144(5):609-618

Oishi K, Amagai N, Shirai H, Kadota K, Ohkura N, Ishida N (2005) Genome-wide expression analysis reveals 100 adrenal gland-dependent circadian genes in the mouse liver. DNA Res 12(3):191-202

Ong HT (2005) The statin studies: from targeting hypercholesterolemia to targeting the high-risk patient. QJM 98(8):599-614

Ostrovsky O, Bengal E, Aronheim A (2002) Induction of terminal differentiation by the c-Jun dimerization protein JDP2 in C2 myoblasts and rhabdomyosarcoma cells. J Biol Chem 277(42):40043-40054

Ostrowski SM, Wilkinson BL, Golde TE, Landreth G (2007) Statins Reduce Amyloid-beta Production through Inhibition of Protein Isoprenylation. J Biol Chem 282(37):26832-26844

Otway DT, Frost G, Johnston JD (2009) Circadian rhythmicity in murine preadipocyte and adipocyte cells. Chronobiol Int 26(7):1340-1354

Panda S, Antoch MP, Miller BH, Su AI, Schook AB, Straume M, Schultz PG, Kay SA, Takahashi JS, Hogenesch JB (2002) Coordinated transcription of key pathways in the mouse by the circadian clock. Cell 109(3):307-320

Parker TS, McNamara DJ, Brown C, Garrigan O, Kolb R, Batwin H, Ahrens EH, Jr. (1982) Mevalonic acid in human plasma: relationship of concentration and circadian rhythm to cholesterol synthesis rates in man. Proc Natl Acad Sci USA 79(9):3037-3041

Petit N, Lescure A, Rederstorff M, Krol A, Moghadaszadeh B, Wewer UM, Guicheney P (2003) Selenoprotein N: an endoplasmic reticulum glycoprotein with an early developmental expression pattern. Hum Mol Genet 12(9):1045-1053

Philips MR (2011) The perplexing case of the geranylgeranyl transferase-deficient mouse. J Clin Invest 121(2):510-513 
Phillips A, Hood SR, Gibson GG, Plant NJ (2005) Impact of transcription factor profile and chromatin conformation on human hepatocyte CYP3A gene expression. Drug Metab Dispos 33(2):233-242

Phillips DC, Hunt JT, Moneypenny CG, Maclean KH, McKenzie PP, Harris LC, Houghton JA (2007) Ceramide-induced G2 arrest in rhabdomyosarcoma (RMS) cells requires p21Cip1/Naf1 induction and is prevented by MDM2 overexpression. Cell Death Differ 14(10):1780-1791

Plakogiannis R, Cohen H (2007) Optimal low-density lipoprotein cholesterol lowering - Morning versus evening statin administration. Ann Pharmacother 41(1):106-110

Plakogiannis R, Cohen H, Taft D (2005) Effects of morning versus evening administration of atorvastatin in patients with hyperlipidemia. Am J Health-Syst Pharm 62(23):2491-2494

Portaluppi F, Smolensky MH (2010) Perspectives on the chronotherapy of hypertension based on the results of the MAPEC study. Chronobiol Int 27(8):16521667

Preitner N, Damiola F, Lopez-Molina L, Zakany J, Duboule D, Albrecht U, Schibler U (2002) The orphan nuclear receptor REV-ERBalpha controls circadian transcription within the positive limb of the mammalian circadian oscillator. Cell 110:251 - 260

Prueksaritanont T, Tang C, Qiu Y, Mu L, Subramanian R, Lin JH (2002) Effects of fibrates on metabolism of statins in human hepatocytes. Drug Metab Dispos 30(11):1280-1287

Puri PL, Wu Z, Zhang P, Wood LD, Bhakta KS, Han J, Feramisco JR, Karin M, Wang JY (2000) Induction of terminal differentiation by constitutive activation of p38 MAP kinase in human rhabdomyosarcoma cells. Genes Dev 14(5):574-584

Recordati, "Recordati Obtains European Licence for Pitavastatin" Press Release, 24 October 2008. (Available from:

http://www.recordati.com/rec en/investors/releases/2008/2008-10-24prl, Accessed 20th November 2008)

Reddy AB, Karp NA, Maywood ES, Sage EA, Deery M, O'Neill JS, Wong GK, Chesham J, Odell M, Lilley KS, Kyriacou CP, Hastings MH (2006) Circadian orchestration of the hepatic proteome. Curr Biol 16(11):1107-1115

Reilly DF, Westgate EJ, FitzGerald GA (2007) Peripheral circadian clocks in the vasculature. Arterioscler Thromb Vasc Biol 27(8):1694-1705 
Reischl S, Vanselow K, Westermark PO, Thierfelder N, Maier B, Herzel H, Kramer A (2007) Beta-TrCP1-mediated degradation of PERIOD2 is essential for circadian dynamics. J Biol Rhythms 22(5):375-386

Ren Z, Elson CE, Gould MN (1997) Inhibition of type I and type II geranylgeranylprotein transferases by the monoterpene perillyl alcohol in NIH3T3 cells. Biochem Pharmacol 54(1):113-120

Reszka AA, Halasy-Nagy J, Rodan GA (2001) Nitrogen-bisphosphonates block retinoblastoma phosphorylation and cell growth by inhibiting the cholesterol biosynthetic pathway in a keratinocyte model for esophageal irritation. $\mathrm{Mol}$ Pharmacol 59(2):193-202

Ridker PM, Danielson E, Fonseca FAH, Genest J, Gotto AM, Jr., Kastelein JJP, Koenig W, Libby P, Lorenzatti AJ, MacFadyen JG, Nordestgaard BG, Shepherd J, Willerson JT, Glynn RJ, the JSG (2008) Rosuvastatin to Prevent Vascular Events in Men and Women with Elevated C-Reactive Protein. N Engl J Med:2195-2207

Romano D, Pertuit M, Rasolonjanahary R, Barnier JV, Magalon K, Enjalbert A, Gerard C (2006) Regulation of the RAP1/RAF-1/extracellularly regulated kinase$1 / 2$ cascade and prolactin release by the phosphoinositide 3-kinase/AKT pathway in pituitary cells. Endocrinology 147(12):6036-6045

Rubin BP, Nishijo K, Chen HI, Yi X, Schuetze DP, Pal R, Prajapati SI, Abraham J, Arenkiel BR, Chen QR, Davis S, McCleish AT, Capecchi MR, Michalek JE, Zarzabal LA, Khan J, Yu Z, Parham DM, Barr FG, Meltzer PS, Chen Y, Keller C (2011) Evidence for an unanticipated relationship between undifferentiated pleomorphic sarcoma and embryonal rhabdomyosarcoma. Cancer cell 19(2):177191

Rusinol AE, Sinensky MS (2006) Farnesylated lamins, progeroid syndromes and farnesyl transferase inhibitors. J Cell Sci 119(Pt 16):3265-3272

Saad B, Dakwar S, Said O, Abu-Hijleh G, Al Battah F, Kmeel A, Aziazeh H (2006) Evaluation of medicinal plant hepatotoxicity in co-cultures of hepatocytes and monocytes. Evid Based Complement Alternat Med 3(1):93-98

Sacheck JM, Ohtsuka A, McLary SC, Goldberg AL (2004) IGF-I stimulates muscle growth by suppressing protein breakdown and expression of atrophyrelated ubiquitin ligases, atrogin-1 and MuRF1. Am J Physiol Endocrinol Metab 287(4):E591-601

Sakamoto K, Honda T, Yokoya S, Waguri S, Kimura J (2007) Rab-small GTPases are involved in fluvastatin and pravastatin-induced vacuolation in rat skeletal myofibers. FASEB J 21(14):4087-4094 
Sakamoto K, Mikami H, Kimura J (2008) Involvement of organic anion transporting polypeptides in the toxicity of hydrophilic pravastatin and lipophilic fluvastatin in rat skeletal myofibres. Br J Pharmacol 154(7):1482-1490

Sakamoto K, Wada I, Kimura J (2011) Inhibition of Rab1 GTPase and Endoplasmic Reticulum-to-Golgi Trafficking Underlies Statin's Toxicity in Rat Skeletal Myofibers. J Pharmacol Exp Ther 338(1):62-69

Sandri M, Sandri C, Gilbert A, Skurk C, Calabria E, Picard A, Walsh K, Schiaffino S, Lecker SH, Goldberg AL (2004) Foxo transcription factors induce the atrophyrelated ubiquitin ligase atrogin-1 and cause skeletal muscle atrophy. Cell 117(3):399-412

Sasaki M, Yoshitane H, Du NH, Okano T, Fukada Y (2009) Preferential inhibition of BMAL2-CLOCK activity by PER2 reemphasizes its negative role and a positive role of BMAL2 in the circadian transcription. J Biol Chem 284(37):25149-25159

Sathasivam S (2012) Statin induced myotoxicity. Eur J Intern Med 23(4):317-324

Scandinavian Simvastatin Survival Study G (1994) Randomised trial of cholesterol lowering in 4444 patients with coronary heart disease: the Scandinavian Simvastatin Survival Study (4S). Lancet 344(8934):1383-1389

Schaars CF, Stalenhoef AF (2008) Effects of ubiquinone (coenzyme Q10) on myopathy in statin users. Curr Opin Lipidol 19(6):553-557

Schiaffino S, Gorza L, Sartore S, Saggin L, Carli M (1986) Embryonic myosin heavy chain as a differentiation marker of developing human skeletal muscle and rhabdomyosarcoma. A monoclonal antibody study. Exp Cell Res 163(1):211-220

Schiaffino S, Mammucari C (2011) Regulation of skeletal muscle growth by the IGF1-Akt/PKB pathway: insights from genetic models. Skelet Muscle 1(1):4

Schmutz I, Ripperger JA, Baeriswyl-Aebischer S, Albrecht U (2010) The mammalian clock component PERIOD2 coordinates circadian output by interaction with nuclear receptors. Genes Dev 24(4):345-357

Schupp N, Schmid U, Heidland A, Stopper H (2008) Rosuvastatin protects against oxidative stress and DNA damage in vitro via upregulation of glutathione synthesis. Atherosclerosis 199(2):278-287

Shepherd J, Cobbe SM, Ford I, Isles CG, Lorimer AR, Macfarlane PW, McKillop $\mathrm{JH}$, Packard CJ, The West of Scotland Coronary Prevention Study G (1995) Prevention of Coronary Heart Disease with Pravastatin in Men with Hypercholesterolemia. N Engl J Med 333(20):1301-1308 
Shi S, Hida A, McGuinness OP, Wasserman DH, Yamazaki S, Johnson CH (2010) Circadian clock gene Bmal1 is not essential; functional replacement with its paralog, Bmal2. Curr Biol 20(4):316-321

Shirogane T, Jin J, Ang XL, Harper JW (2005) SCFbeta-TRCP controls clockdependent transcription via casein kinase 1-dependent degradation of the mammalian period-1 (Per1) protein. J Biol Chem 280(29):26863-26872

Shitara Y, Sugiyama $Y$ (2006) Pharmacokinetic and pharmacodynamic alterations of 3-hydroxy-3-methylglutaryl coenzyme A (HMG-CoA) reductase inhibitors: drugdrug interactions and interindividual differences in transporter and metabolic enzyme functions. Pharmacol Ther 112(1):71-105

Shukla P, Gupta D, Munshi A, Agarwal JP (2011) Cetuximab and cancers of the head and neck: tapping the circadian rhythm. Med Hypotheses 77(3):336-338

Sidaway J, Wang Y, Marsden AM, Orton TC, Westwood FR, Azuma CT, Scott RC (2009) Statin-induced myopathy in the rat: relationship between systemic exposure, muscle exposure and myopathy. Xenobiotica 39(1):90-98

Siddals KW, Marshman E, Westwood M, Gibson JM (2004) Abrogation of insulinlike growth factor-I (IGF-I) and insulin action by mevalonic acid depletion: synergy between protein prenylation and receptor glycosylation pathways. J Biol Chem 279(37):38353-38359

Sieczkowski E, Lehner C, Ambros PF, Hohenegger M (2010) Double impact on pglycoprotein by statins enhances doxorubicin cytotoxicity in human neuroblastoma cells. Int J Cancer 126(9):2025-2035

Siepka SM, Yoo S-H, Park J, Song W, Kumar V, Hu Y, Lee C, Takahashi JS (2007) Circadian Mutant Overtime Reveals F-box Protein FBXL3 Regulation of Cryptochrome and Period Gene Expression. Cell 129(5):1011-1023

Sigma-Aldrich, "Product Information: FTI-277 trifluoroacetate salt" 2011 a. (Avaliable from:

http://www.sigmaaldrich.com/etc/medialib/docs/Sigma/Product Information Sheet/ f9803pis.Par.0001.File.tmp/f9803pis.pdf, Accessed 19th July 2011)

Sigma-Aldrich, "Product Information: GGTI-2133" 2011b. (Avallable from: http://www.sigmaaldrich.com/catalog/ProductDetail.do?lang=en\&N4=G5294/SIGM A\&N5=SEARCH CONCAT PNO|BRAND KEY\&F=SPEC, Accessed 19th July 2011)

Sinensky M, Fantle K, Dalton M (1994) An antibody which specifically recognizes prelamin A but not mature lamin A: application to detection of blocks in farnesylation-dependent protein processing. Cancer Res 54(12):3229-3232 
Sirvent P, Mercier J, Vassort G, Lacampagne A (2005) Simvastatin triggers mitochondria-induced $\mathrm{Ca} 2+$ signaling alteration in skeletal muscle. Biochem Biophys Res Commun 329(3):1067-1075

Sjogren AK, Andersson KM, Khan O, Olofsson FJ, Karlsson C, Bergo MO (2011) Inactivating GGTase-I reduces disease phenotypes in a mouse model of K-RASinduced myeloproliferative disease. Leukemia 25(1):186-189

Spindler SR, Li R, Dhahbi JM, Yamakawa A, Mote P, Bodmer R, Ocorr K, Williams RT, Wang Y, Ablao KP (2012) Statin treatment increases lifespan and improves cardiac health in Drosophila by decreasing specific protein prenylation. PLoS One 7(6):e39581

Stadtman TC (1996) Selenocysteine. Annu Rev Biochem 65(1):83-100

Staffa JA, Chang J, Green L (2002) Cerivastatin and Reports of Fatal Rhabdomyolysis. N Engl J Med 346(7):539-540

Stagnitti MN, "Trends in Statins Utilization and Expenditures for the U.S. Civilian Noninstitutionalized Population, 2000 and 2005" 2008. (Available from: http://www.meps.ahrq.gov/mepsweb/data files/publications/st205/stat205.pdf, Accessed 5th November 2008)

Steinberg D (2006) An interpretive history of the cholesterol controversy, part V: The discovery of the statins and the end of the controversy. J Lipid Res 47(7):1339-1351

Storch KF, Lipan O, Leykin I, Viswanathan N, Davis FC, Wong WH, Weitz CJ (2002) Extensive and divergent circadian gene expression in liver and heart. Nature 417:78 - 82

Stork PJ (2003) Does Rap1 deserve a bad Rap? Trends Biochem Sci 28(5):267275

Stossel TP (2008) The discovery of statins. Cell 134(6):903-905

Sutter AP, Maaser K, Hopfner M, Huether A, Schuppan D, Scherubl H (2005) Cell cycle arrest and apoptosis induction in hepatocellular carcinoma cells by HMGCoA reductase inhibitors. Synergistic antiproliferative action with ligands of the peripheral benzodiazepine receptor. J Hepatol 43(5):808-816

Tahara Y, Kuroda H, Saito K, Nakajima Y, Kubo Y, Ohnishi N, Seo Y, Otsuka M, Fuse Y, Ohura Y, Komatsu T, Moriya Y, Okada S, Furutani N, Hirao A, Horikawa K, Kudo T, Shibata S (2012) In Vivo monitoring of peripheral circadian clocks in the mouse. Curr Biol 22(11):1029-1034 
Takahashi JS, Hong HK, Ko CH, McDearmon EL (2008) The genetics of mammalian circadian order and disorder: implications for physiology and disease. Nat Rev Genet 9(10):764-775

Takano A, Isojima $\mathrm{Y}$, Nagai K (2004) Identification of mPer1 phosphorylation sites responsible for the nuclear entry. J Biol Chem 279(31):32578-32585

Takeda, "Discontinuation of development of TAK-475, a compound for treatment of hypercholesterolemia" Press Release, 28 March 2008. (Avaliable from: http://www.takeda.com/press/article 29153.html, Accessed 26th November 2008)

Takeda M, Noshiro R, Onozato ML, Tojo A, Hasannejad H, Huang XL, Narikawa S, Endou $\mathrm{H}$ (2004) Evidence for a role of human organic anion transporters in the muscular side effects of HMG-CoA reductase inhibitors. Eur J Pharmacol 483(23):133-138

Takiguchi T, Tomita M, Matsunaga N, Nakagawa H, Koyanagi S, Ohdo S (2007) Molecular basis for rhythmic expression of CYP3A4 in serum-shocked HepG2 cells. Pharmacogenet Genomics 17(12):1047-1056

Tanaka S, Sakamoto K, Yamamoto M, Mizuno A, Ono T, Waguri S, Kimura J (2010) Mechanism of statin-induced contractile dysfunction in rat cultured skeletal myofibers. J Pharmacol Sci 114(4):454-463

Tapscott SJ, Thayer MJ, Weintraub H (1993) Deficiency in Rhabdomyosarcomas of a Factor Required for Myod Activity and Myogenesis. Science 259(5100):14501453

Tavridou A, Kaklamanis L, Megaritis G, Kourounakis AP, Papalois A, Roukounas D, Rekka EA, Kourounakis PN, Charalambous A, Manolopoulos VG (2006) Pharmacological characterization in vitro of EP2306 and EP2302, potent inhibitors of squalene synthase and lipid biosynthesis. Eur J Pharmacol 535(1-3):34-42

Taylor F, Ward K, Moore TH, Burke M, Davey Smith G, Casas JP, Ebrahim S (2011) Statins for the primary prevention of cardiovascular disease. Cochrane Database Syst Rev(1):CD004816

Terry KL, Casey PJ, Beese LS (2006) Conversion of protein farnesyltransferase to a geranylgeranyltransferase. Biochemistry 45(32):9746-9755

Tidyman WE, Lee HS, Rauen KA (2011) Skeletal muscle pathology in Costello and cardio-facio-cutaneous syndromes: developmental consequences of germline Ras/MAPK activation on myogenesis. Am J Med Genet C Semin Med Genet 157(2):104-114

Tokui T, Nakai D, Nakagomi R, Yawo H, Abe T, Sugiyama Y (1999) Pravastatin, an HMG-CoA Reductase Inhibitor, Is Transported by Rat Organic Anion

Transporting Polypeptide, oatp2. Pharm Res 16(6):904-908 
Tong $\mathrm{H}$, Holstein SA, Hohl RJ (2005) Simultaneous determination of farnesyl and geranylgeranyl pyrophosphate levels in cultured cells. Anal Biochem 336(1):51-59

Tracey CJ, Pan X, Catterson JH, Harmar AJ, Hussain MM, Hartley PS (2012) Diurnal expression of Thrombopoietin is regulated by Clock. $J$ Thromb Haemost:DOI: 10.1111/j.1538-7836.2012.04643.x

Traustadottir T, Stock AA, Harman SM (2008) High-dose statin use does not impair aerobic capacity or skeletal muscle function in older adults. Age 30(4):283291

Ueda S, Kitazawa S, Ishida K, Nishikawa Y, Matsui M, Matsumoto H, Aoki T, Nozaki S, Takeda T, Tamori Y, Aiba A, Kahn CR, Kataoka T, Satoh T (2010) Crucial role of the small GTPase Rac1 in insulin-stimulated translocation of glucose transporter 4 to the mouse skeletal muscle sarcolemma. FASEB $\mathrm{J}$ 24(7):2254-2261

Vachon PH, Loechel F, Xu H, Wewer UM, Engvall E (1996) Merosin and laminin in myogenesis; specific requirement for merosin in myotube stability and survival. $J$ Cell Biol 134(6):1483-1497

Vaklavas C, Chatzizisis YS, Ziakas A, Zamboulis C, Giannoglou GD (2009) Molecular basis of statin-associated myopathy. Atherosclerosis 202(1):18-28

Van Kolen K, Gilany K, Moens L, Esmans EL, Slegers H (2006) P2Y12 receptor signalling towards $\mathrm{PKB}$ proceeds through IGF-I receptor cross-talk and requires activation of Src, Pyk2 and Rap1. Cell Signal 18(8):1169-1181

Vandesompele J, De Preter K, Pattyn F, Poppe B, Van Roy N, De Paepe A, Speleman F (2002) Accurate normalization of real-time quantitative RT-PCR data by geometric averaging of multiple internal control genes. Genome Biol 3(7):research0034.0031 - research0034.0011

Vielhaber E, Eide E, Rivers A, Gao ZH, Virshup DM (2000) Nuclear entry of the circadian regulator $\mathrm{mPER} 1$ is controlled by mammalian casein kinase I epsilon. $\mathrm{Mol}$ Cell Biol 20(13):4888-4899

Virshup DM, Forger DB (2007) After Hours Keeps Clock Researchers CRYing Overtime. Cell 129(5):857-859

Voet D, Voet JG; Biochemistry, 3rd Edition. 2004, Wiley International

Vojtek AB, Der CJ (1998) Increasing complexity of the Ras signaling pathway. J Biol Chem 273(32):19925-19928

Voora D, Shah SH, Spasojevic I, Ali S, Reed CR, Salisbury BA, Ginsburg GS (2009) The SLCO1B1*5 genetic variant is associated with statin-induced side effects. J Am Coll Cardiol 54(17):1609-1616 
Wagner BK, Gilbert TJ, Hanai J, Imamura S, Bodycombe NE, Bon RS, Waldmann $\mathrm{H}$, Clemons PA, Sukhatme VP, Mootha VK (2011) A small-molecule screening strategy to identify suppressors of statin myopathy. ACS Chem Biol 6(9):900-904

Wang H, Liu D, Cao P, Lecker S, Hu Z (2010a) Atrogin-1 affects muscle protein synthesis and degradation when energy metabolism is impaired by the antidiabetes drug berberine. Diabetes 59(8):1879-1889

Wang L, Liu F, Adamo ML (2001) Cyclic AMP inhibits extracellular signalregulated kinase and phosphatidylinositol 3-kinase/Akt pathways by inhibiting Rap1. J Biol Chem 276(40):37242-37249

Wang S, Guo L, Dong L, Li S, Zhang J, Sun M (2010b) TGF-beta1 signal pathway may contribute to rhabdomyosarcoma development by inhibiting differentiation. Cancer Sci 101(5):1108-1116

Warner GJ, Berry MJ, Moustafa ME, Carlson BA, Hatfield DL, Faust JR (2000) Inhibition of selenoprotein synthesis by selenocysteine tRNA[Ser]Sec lacking isopentenyladenosine. J Biol Chem 275(36):28110-28119

Welsh DK, Yoo SH, Liu AC, Takahashi JS, Kay SA (2004) Bioluminescence imaging of individual fibroblasts reveals persistent, independently phased circadian rhythms of clock gene expression. Curr Biol 14(24):2289-2295

Weng TC, Yang YH, Lin SJ, Tai SH (2010) A systematic review and metaanalysis on the therapeutic equivalence of statins. $J$ Clin Pharm Ther 35(2):139151

Westwood FR, Scott RC, Marsden AM, Bigley A, Randall K (2008) Rosuvastatin: characterization of induced myopathy in the rat. Toxicol Pathol 36(2):345-352

Whyte DB, Kirschmeier P, Hockenberry TN, Nunez-Oliva I, James L, Catino JJ, Bishop WR, Pai JK (1997) K- and N-Ras are geranylgeranylated in cells treated with farnesyl protein transferase inhibitors. J Biol Chem 272(22):14459-14464

Wilson KL (2000) The nuclear envelope, muscular dystrophy and gene expression. Trends Cell Biol 10(4):125-129

World Health Organisation, "The Global Burden of Disease 2004 Update" 2008. (Available from:

http://www.who.int/healthinfo/global burden disease/GBD report 2004update full .pdf, Accessed 21st November 2008)

Wu T, Ni Y, Kato H, Fu Z (2010) Feeding-induced rapid resetting of the hepatic circadian clock is associated with acute induction of Per2 and Dec1 transcription in rats. Chronobiol Int 27(1):1-18 
Wu X, Zvonic S, Floyd ZE, Kilroy G, Goh BC, Hernandez TL, Eckel RH, Mynatt RL, Gimble JM (2007) Induction of circadian gene expression in human subcutaneous adipose-derived stem cells. Obesity (Silver Spring) 15(11):2560-2570

Xia Z, Tan MM, Wong WW, Dimitroulakos J, Minden MD, Penn LZ (2001) Blocking protein geranylgeranylation is essential for lovastatin-induced apoptosis of human acute myeloid leukemia cells. Leukemia 15(9):1398-1407

Xiao R, Ferry AL, Dupont-Versteegden EE (2011) Cell death-resistance of differentiated myotubes is associated with enhanced anti-apoptotic mechanisms compared to myoblasts. Apoptosis 16(3):221-234

Xu Y, Padiath QS, Shapiro RE, Jones CR, Wu SC, Saigoh N, Saigoh K, Ptacek LJ, Fu YH (2005) Functional consequences of a CKIdelta mutation causing familial advanced sleep phase syndrome. Nature 434(7033):640-644

Yaktubay Dondas N, Sucu N, Coskun Yilmaz B, Kaplan HM, Ozeren M, Karaca MK, Vezir O, Singirik E (2011) Molecular mechanism of vasorelaxant and antiatherogenic effects of the statins in the human saphenous vein graft. Eur $J$ Pharmacol 666(1-3):150-157

Yamaguchi S, Mitsui S, Yan L, Yagita K, Miyake S, Okamura H (2000) Role of DBP in the circadian oscillatory mechanism. Mol Cell Biol 20(13):4773-4781

Yamajuku D, Inagaki T, Haruma T, Okubo S, Kataoka Y, Kobayashi S, Ikegami K, Laurent T, Kojima T, Noutomi K, Hashimoto S, Oda H (2012) Real-time monitoring in three-dimensional hepatocytes reveals that insulin acts as a synchronizer for liver clock. Sci Rep 2:439

Yamazaki S, Numano R, Abe M, Hida A, Takahashi R-i, Ueda M, Block GD, Sakaki Y, Menaker M, Tei H (2000) Resetting Central and Peripheral Circadian Oscillators in Transgenic Rats. Science 288(5466):682-685

Yang SH, Chang SY, Tu Y, Lawson GW, Bergo MO, Fong LG, Young SG (2012) Severe hepatocellular disease in mice lacking one or both CaaX prenyltransferases. J Lipid Res 53(1):77-86

Yang ZH, MacQuarrie KL, Analau E, Tyler AE, Dilworth FJ, Cao Y, Diede SJ, Tapscott SJ (2009) MyoD and E-protein heterodimers switch rhabdomyosarcoma cells from an arrested myoblast phase to a differentiated state. Genes Dev 23(6):694-707

Yoo SH, Yamazaki S, Lowrey PL, Shimomura K, Ko CH, Buhr ED, Siepka SM, Hong HK, Oh WJ, Yoo OJ, Menaker M, Takahashi JS (2004)

PERIOD2::LUCIFERASE real-time reporting of circadian dynamics reveals persistent circadian oscillations in mouse peripheral tissues. Proc Natl Acad Sci USA 101(15):5339-5346 
Zhang X, Dube TJ, Esser KA (2009) Working around the clock: circadian rhythms and skeletal muscle. J App/ Physiol 107(5):1647-1654

Zhang X, Patel SP, McCarthy JJ, Rabchevsky AG, Goldhamer DJ, Esser KA (2012) A non-canonical E-box within the MyoD core enhancer is necessary for circadian expression in skeletal muscle. Nucleic Acids Res 40(8):3419-3430

Zmrzljak UP, Rozman D (2012) Circadian regulation of the hepatic endobiotic and xenobitoic detoxification pathways: the time matters. Chem Res Toxicol 25(4):811824

Zvonic S, Ptitsyn AA, Conrad SA, Scott LK, Floyd ZE, Kilroy G, Wu X, Goh BC, Mynatt RL, Gimble JM (2006) Characterization of peripheral circadian clocks in adipose tissues. Diabetes 55(4):962-970 


\section{Appendices}

\subsection{Normalisation of Temporal Gene Expression Data from Pilot Serum Pulse Experiment}

\subsubsection{T3-L1 Murine Preadipocyte Cell Line}

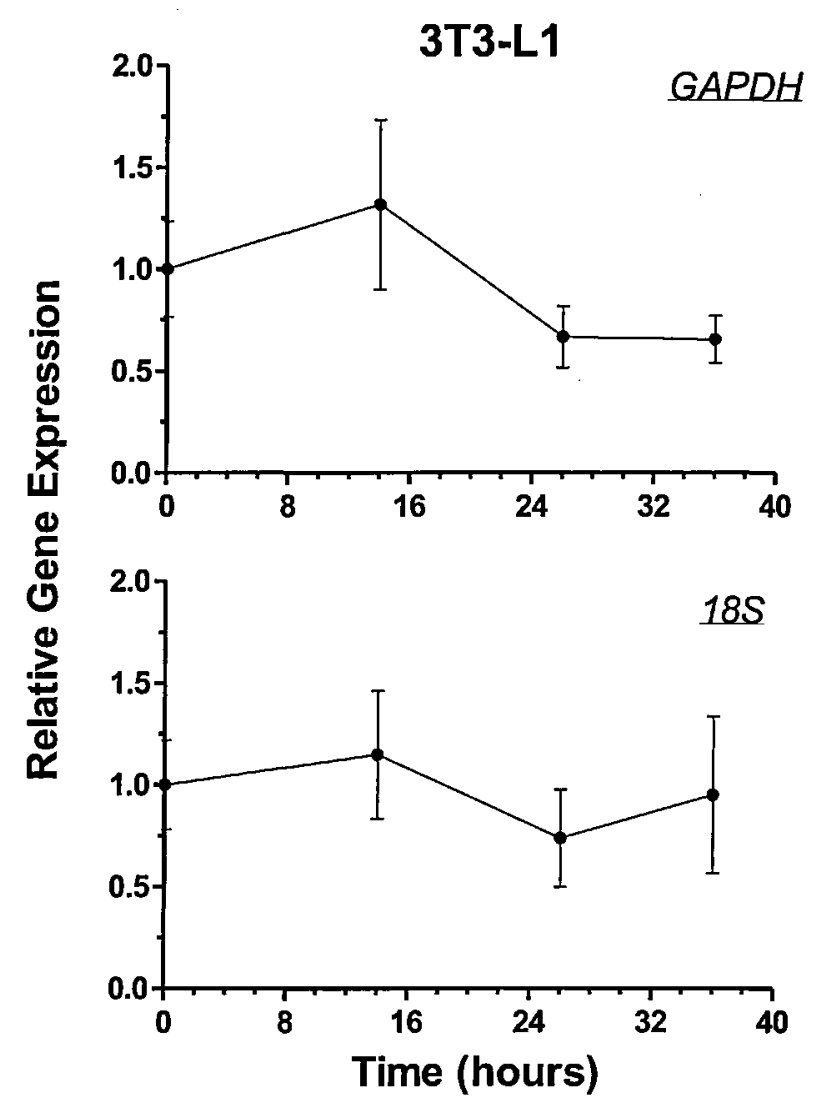

Figure 8-1 Temporal gene expression of two different housekeeping genes in 3T3-L1 cells 3T3-L1 cells were synchronised using a serum pulse (at $T=0$ ) and RNA was extracted at $T=0,14$, 26 and 36. Temporal gene expression of housekeeping genes glyceraldehyde 3-phosphate dehydrogenase (GAPDH) and $18 \mathrm{~S}$ was measured by quantitative RT-PCR (TaqMan). Data are presented as mean \pm standard deviation (SD), relative to expression at $T=0$, from a single experiment where $\mathrm{N}=4$. 
3T3-L1
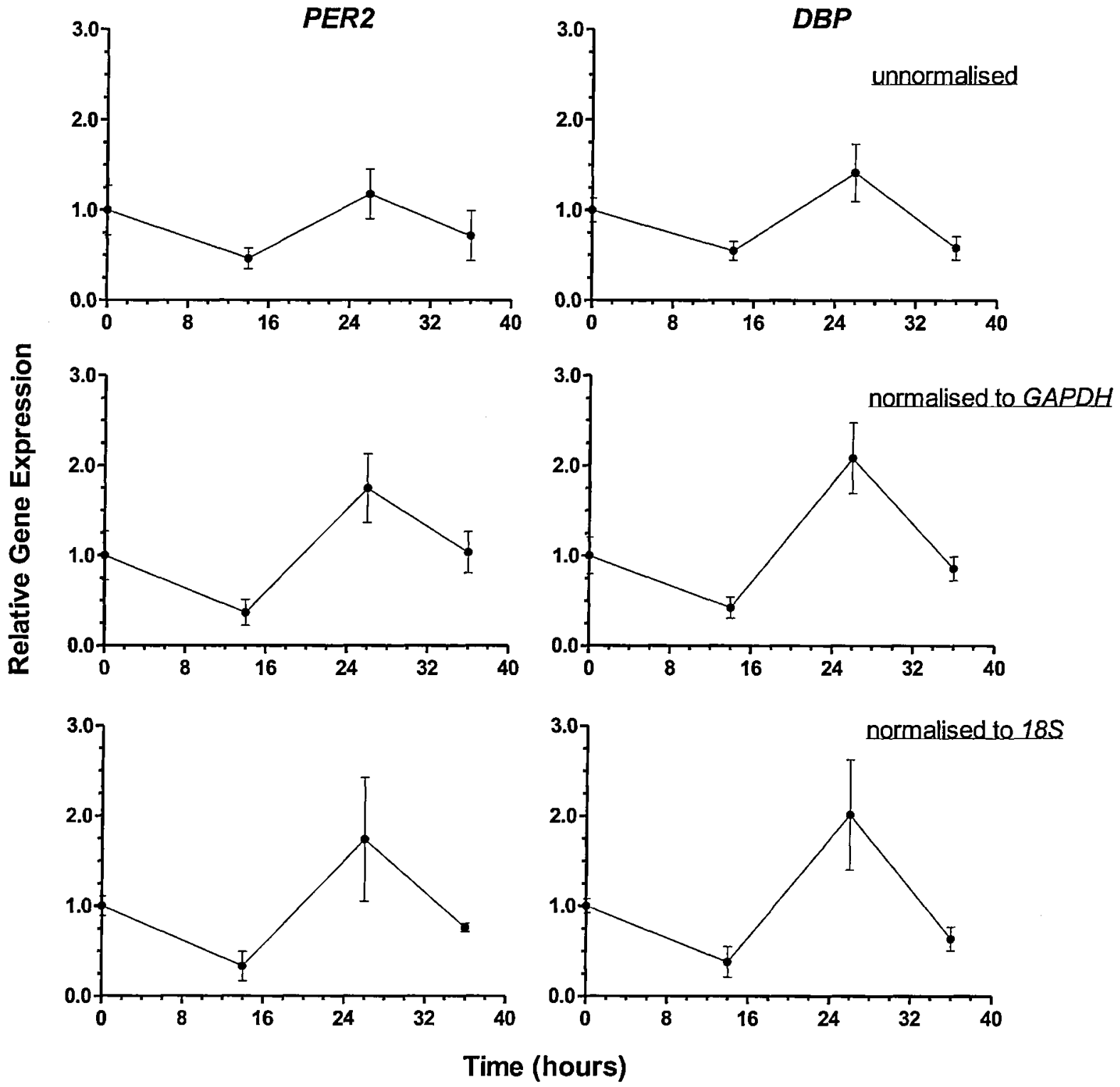

Figure 8-2 Effect of normalisation of 3T3-L1 TaqMan data using two different housekeeping genes

3T3-L1 cells were synchronised using a serum pulse (at $T=0$ ) and temporal gene expression of PER2 and DBP was measured by quantitative RT-PCR (TaqMan). Data are shown unnormalised and normalised to GAPDH or $18 \mathrm{~S}$ expression, and are presented as mean \pm standard deviation $(S D)$, relative to expression at $T=0$, from a single experiment where $N=4$. 


\subsubsection{HepG2 and Huh7 Human Liver Cell Lines}
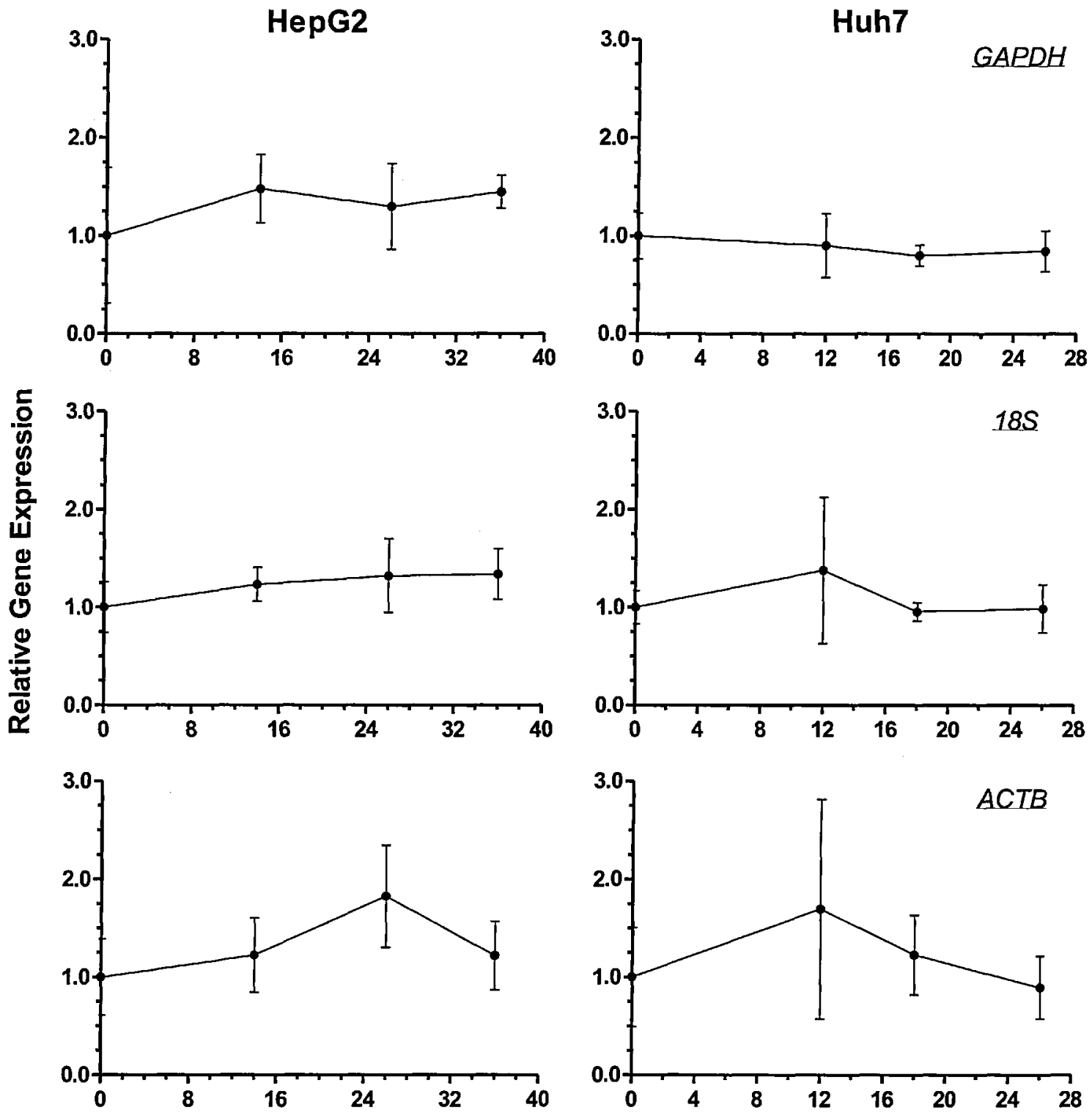

Time (hours)

Figure 8-3 Temporal gene expression of three different housekeeping genes in HepG2 and Huh7 cells

HepG2 and Huh7 cells were synchronised using a serum pulse (at $T=0$ ). RNA was extracted from HepG2 cells at $T=0,14,26,36$ and from Huh7 cells at $T=0,12,18$ and 26 . Temporal gene expression of housekeeping genes $G A P D H, 18 S$ and $A C T B$ was measured by quantitative RTPCR (TaqMan). Data are presented as mean \pm standard deviation (SD), relative to expression at $T=0$, from a single experiment where $N=4$. 
HepG2
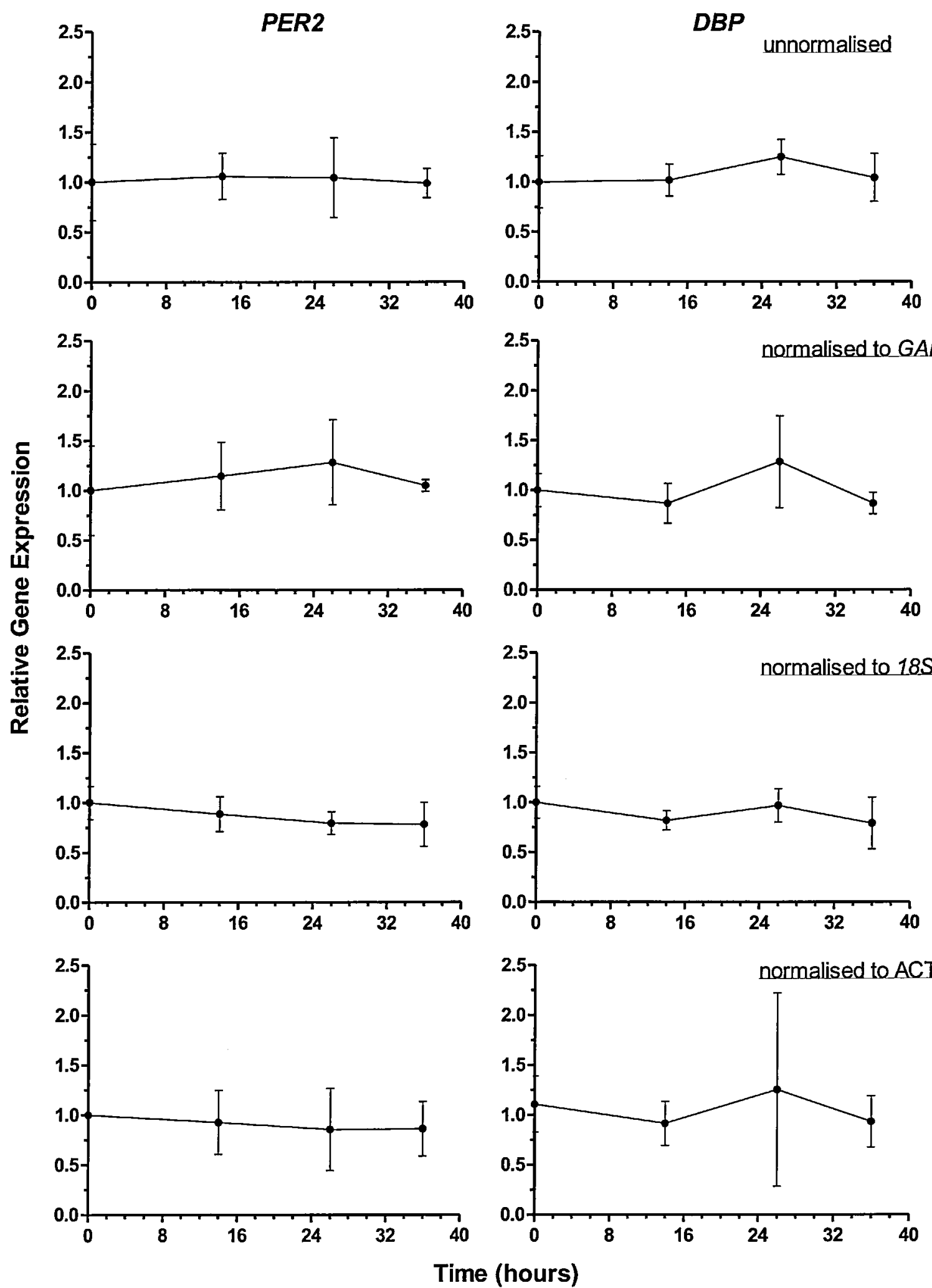

Figure 8-4 Effect of normalisation of HepG2 TaqMan data using three different housekeeping genes

HepG2 cells were synchronised using a serum pulse (at $T=0$ ) and temporal gene expression of $P E R 2$ and $D B P$ was measured by quantitative RT-PCR (TaqMan). Data are shown unnormalised and normalised to $G A P D H, 18 S$ or $A C T B$ expression, and are presented as mean \pm standard deviation (SD), relative to expression at $T=0$, from a single experiment where $N=4$. 
Huh7
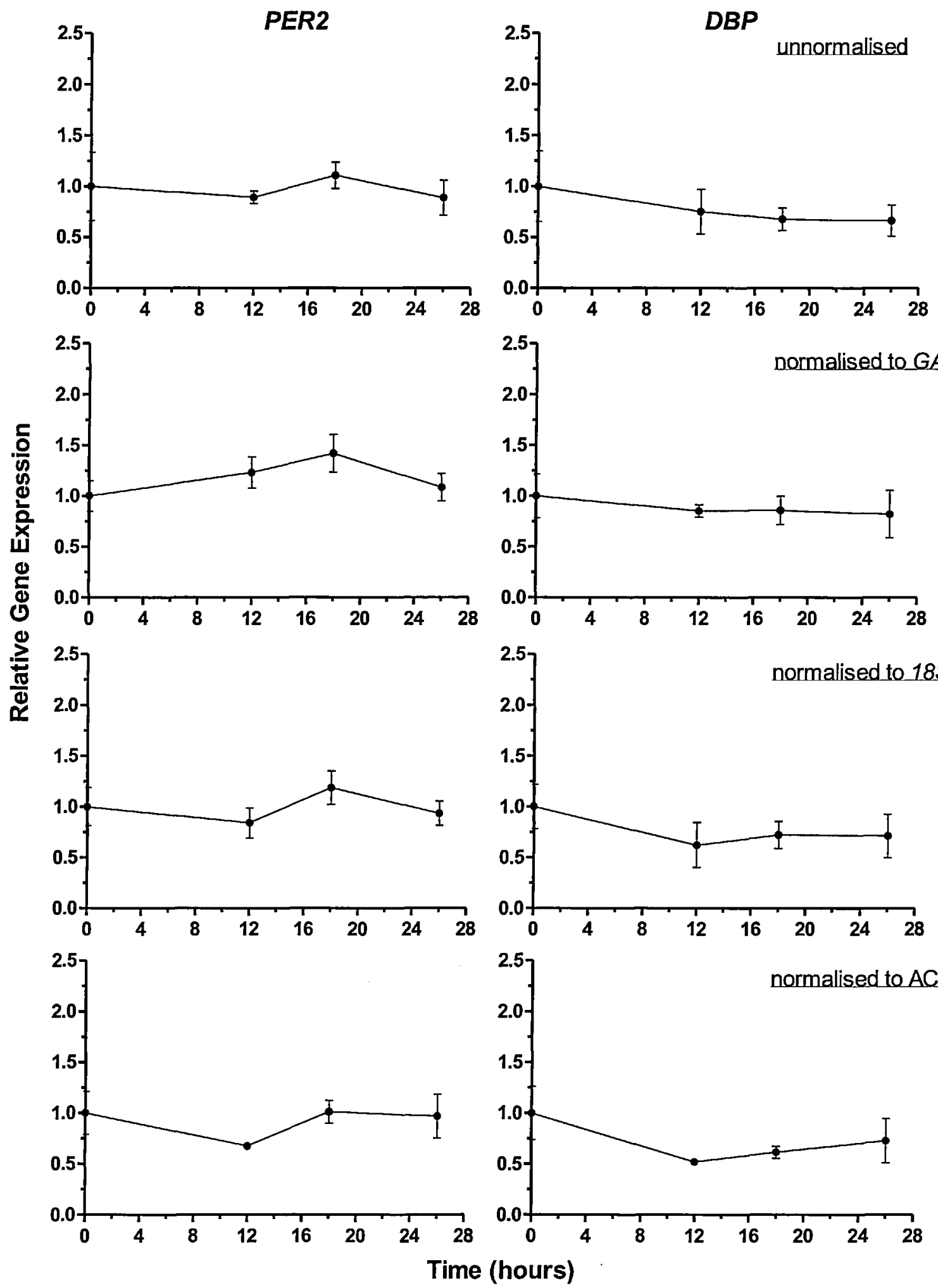

Figure 8-5 Effect of normalisation of Huh7 TaqMan data using three different housekeeping genes

Huh7 cells were synchronised using a serum pulse (at $\mathrm{T}=0$ ) and temporal gene expression of $P E R 2$ and $D B P$ was measured by quantitative RT-PCR (TaqMan). Data are shown unnormalised and normalised to $G A P D H, 18 S$ or $A C T B$ expression, and are presented as mean \pm standard deviation (SD), relative to expression at $T=0$, from a single experiment where $N=4$. 


\subsection{Additional Normalisations of Temporal Gene Expression Data from Circadian Analysis in Huh7 and RD Cells}

\subsubsection{Normalisation of Temporal Gene Expression Data from Huh7 Cells}

Huh7

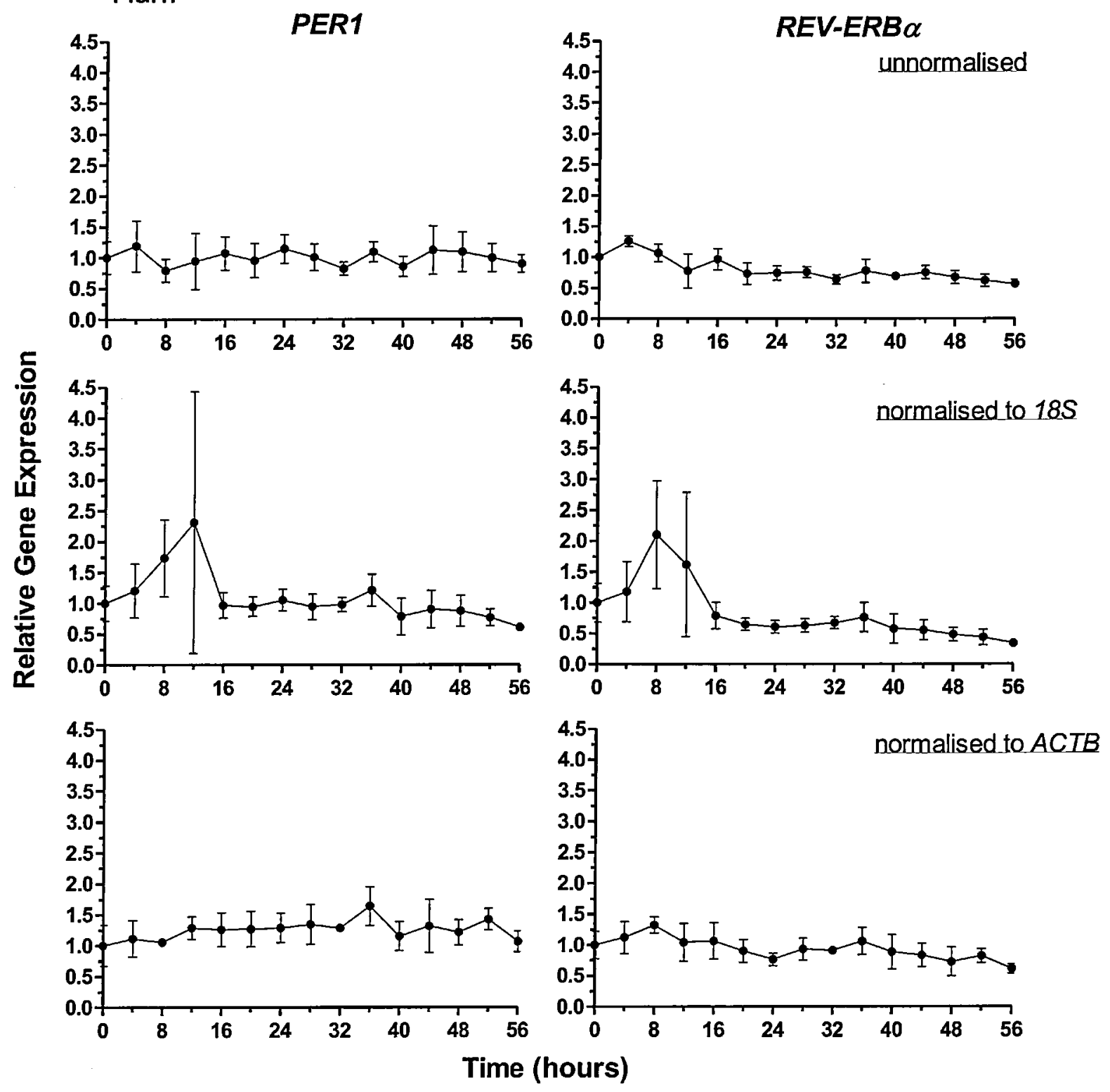

Figure 8-6 Effect of normalisation of Huh7 TaqMan data (PER1 and REV-ERBa) using two different housekeeping genes

Huh7 cells were synchronised using a serum pulse (at $\mathrm{T}=0$ ) and temporal gene expression of PER1 and REV-ERBa was measured by quantitative RT-PCR (TaqMan). Data are shown unnormalised and normaiised to $18 \mathrm{~S}$ or $A C T B$ expression, and are presented as mean \pm standard deviation (SD), relative to expression at $\mathrm{T}=0$, from a single experiment where $\mathrm{N}=4$. 


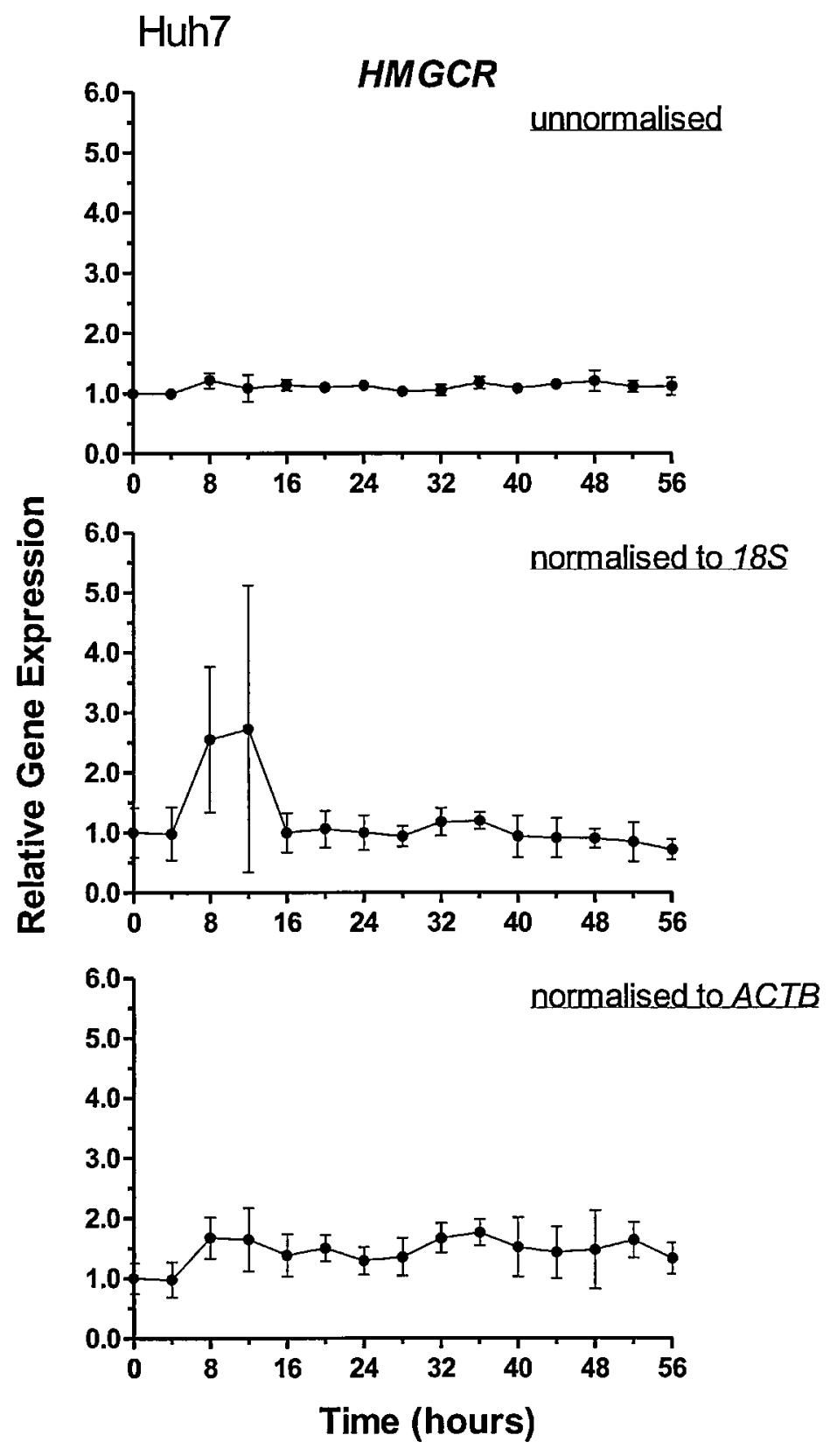

Figure 8-7 Effect of normalisation of Huh7 TaqMan data (HMGCR) using two different housekeeping genes

Huh7 cells were synchronised using a serum pulse (at $T=0$ ) and temporal gene expression of HMGCR was measured by quantitative RT-PCR (TaqMan). Data are shown unnormalised and normalised to $18 S$ or ACTB expression, and are presented as mean \pm standard deviation (SD), relative to expression at $\mathrm{T}=0$, from a single experiment where $\mathrm{N}=4$. 


\subsubsection{Normalisation of Temporal Gene Expression Data from RD-D Cells}

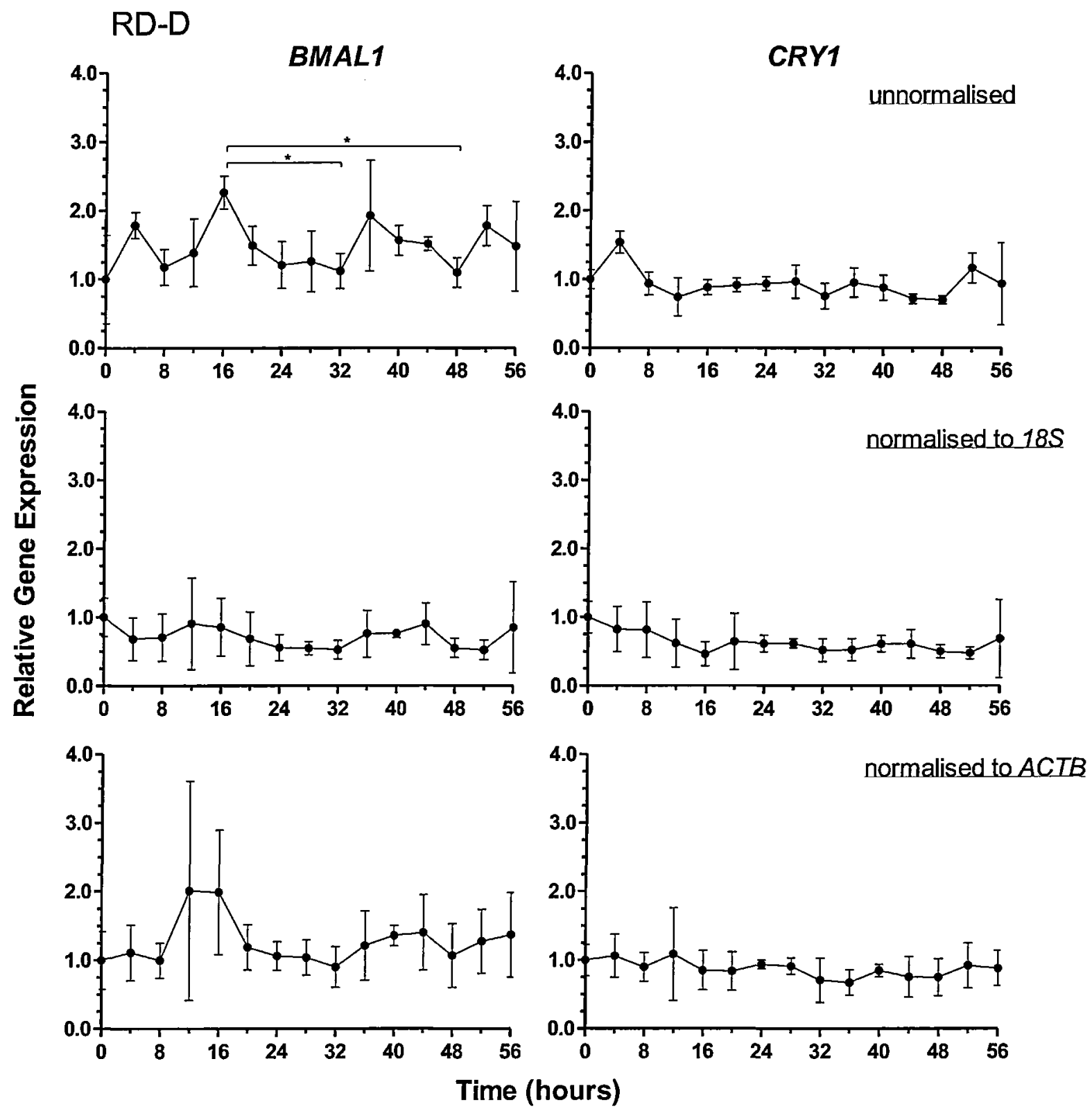

Figure 8-8 Effect of normalisation of RD-D TaqMan data (BMAL1 and CRY1) using two different housekeeping genes

$R D-D$ cells were synchronised using a serum pulse (at $T=0$ ) and temporal gene expression of BMAL1 and CRY1 was measured by quantitative RT-PCR (TaqMan). Data are shown unnormalised and normalised to $18 S$ or $A C T B$ expression, and are presented as mean \pm standard deviation $(S D)$, relative to expression at $T=0$, from a single experiment where $N=4$. Statistical significance determined by one-way ANOVA followed by Bonferroni post hoc test: ${ }^{*}=\mathrm{P}<0.05$. 


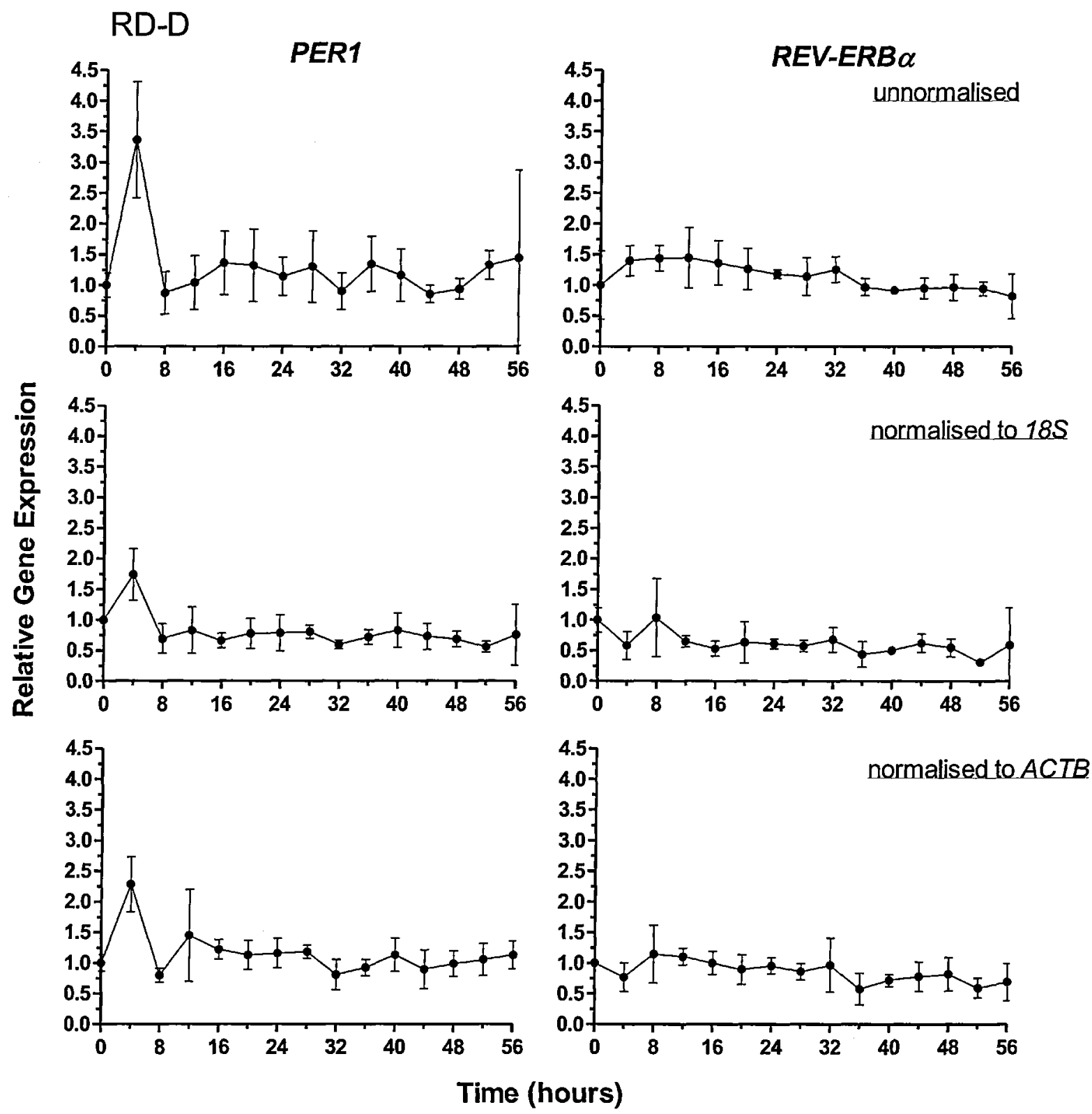

Figure 8-9 Effect of normalisation of RD-D TaqMan data (PER1 and REV-ERBa) using two different housekeeping genes

RD-D cells were synchronised using a serum pulse (at $T=0$ ) and temporal gene expression of PER1 and REV-ERBa was measured by quantitative RT-PCR (TaqMan). Data are shown unnormalised and normalised to $18 S$ or $A C T B$ expression, and are presented as mean \pm standard deviation (SD), reiative to expression at $\mathrm{T}=0$, from a single experiment where $\mathrm{N}=4$. 


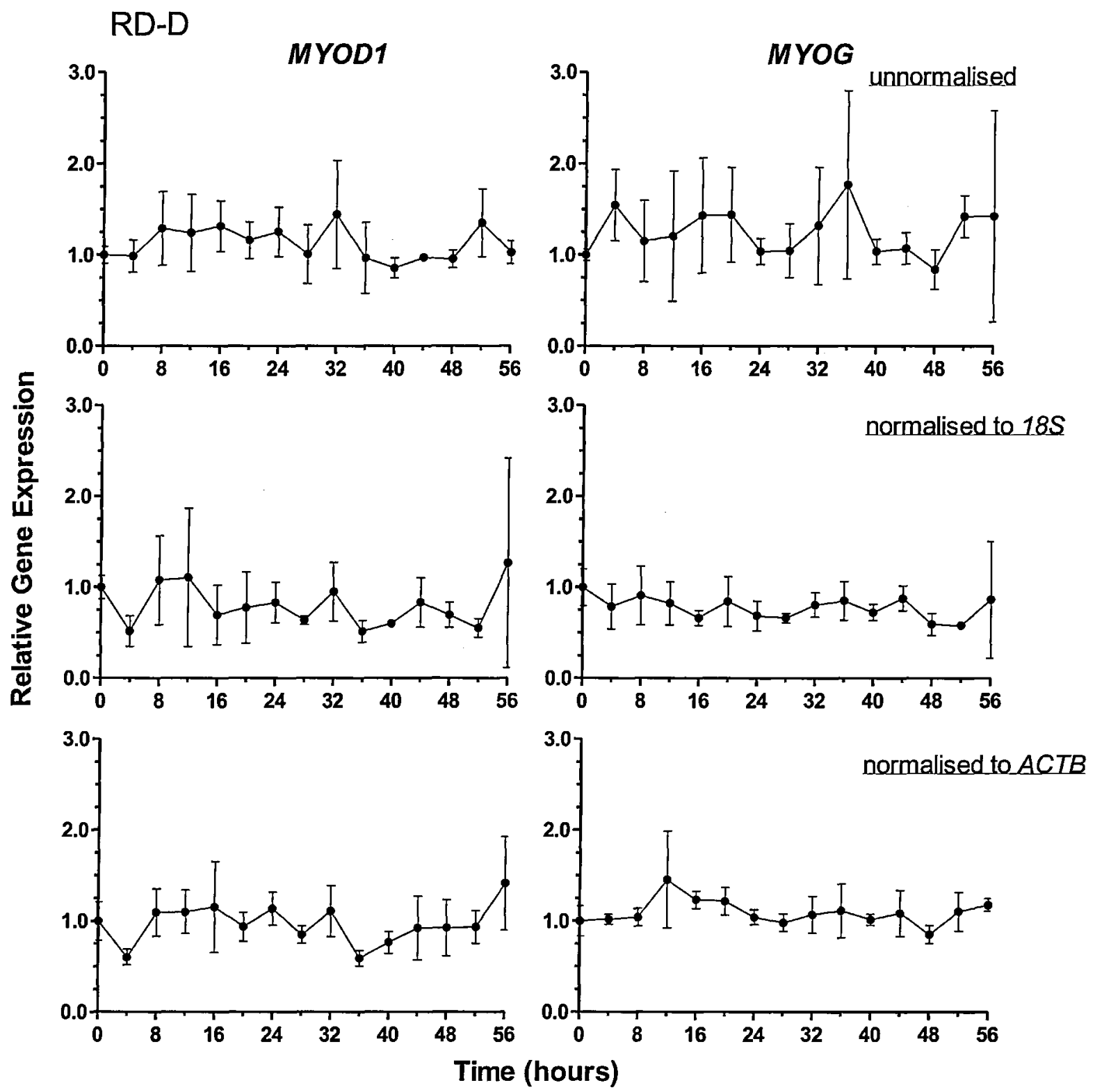

Figure 8-10 Effect of normalisation of RD-D TaqMan data (MYOD1 and MYOG) using two different housekeeping genes

RD-D cells were synchronised using a serum pulse (at $T=0$ ) and temporal gene expression of MYOD1 and MYOG was measured by quantitative RT-PCR (TaqMan). Data are shown unnormalised and normalised to $18 S$ or ACTB expression, and are presented as mean \pm standard deviation (SD), relative to expression at $T=0$, from a single experiment where $N=4$. 


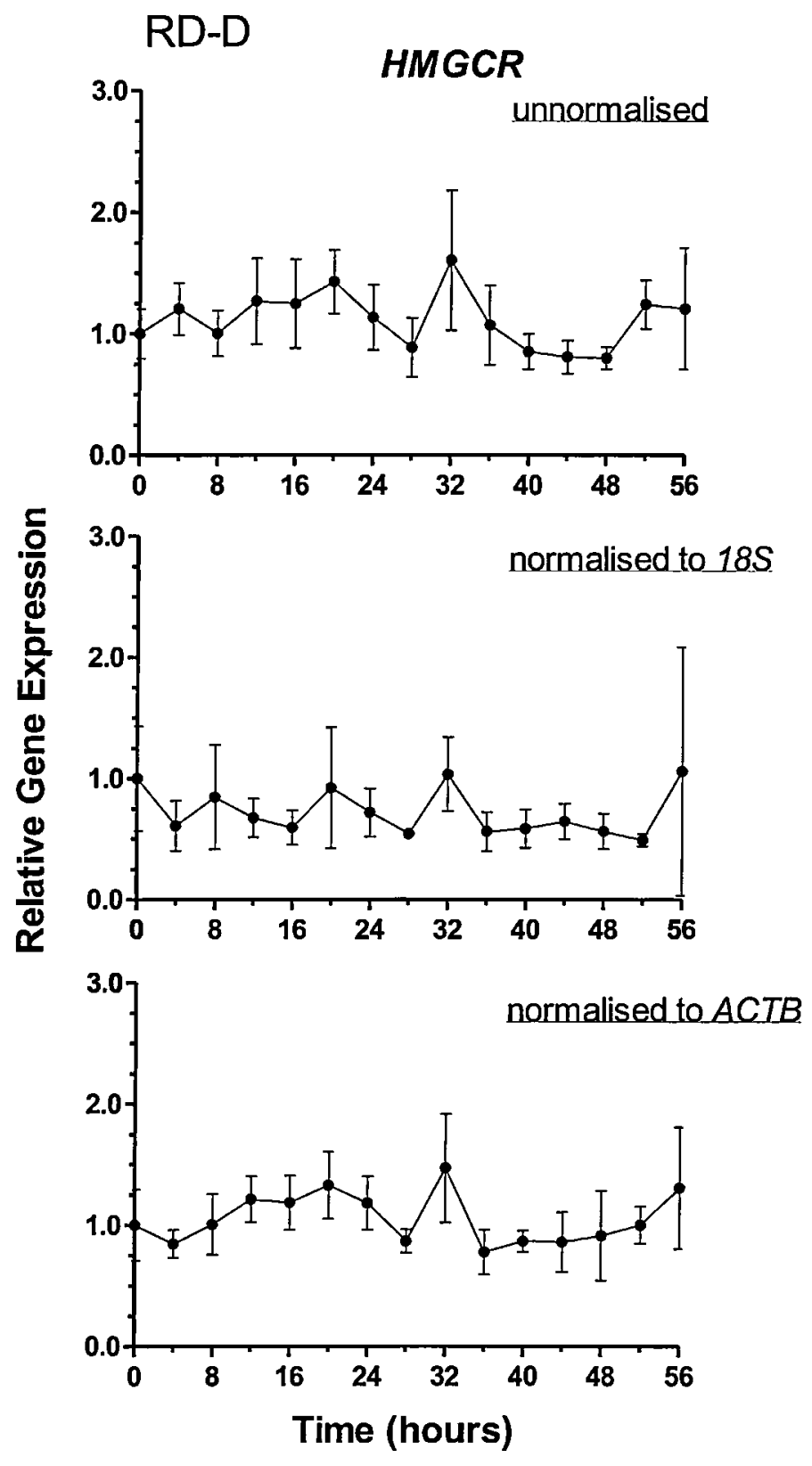

Figure 8-11 Effect of normalisation of RD-D TaqMan data (HMGCR) using two different housekeeping genes

RD-D cells were synchronised using a serum pulse (at $T=0$ ) and temporal gene expression of HMGCR was measured by quantitative RT-PCR (TaqMan). Data are shown unnormalised and normalised to $18 \mathrm{~S}$ or $A C T B$ expression, and are presented as mean \pm standard deviation (SD), relative to expression at $T=0$, from a single experiment where $N=4$. 


\subsection{Conference Presentations}

2009 - University of Surrey FHMS Annual Festival of Research (poster)

2010 - British Toxicology Society (BTS) Annual Congress 2010 (poster)

- University of Surrey FHMS Annual Festival of Research (poster)

2011 - BTS Annual Congress (poster, commended)

- University of Surrey FHMS Annual Festival of Research (poster)

2012 - BTS Annual Congress (oral presentation)

- Joint $19^{\text {th }}$ International Symposium on Microsomes and Drug Oxidations (MDO) and $12^{\text {th }}$ European meeting of the International Society for the Study of Xenobiotics (ISSX) (poster)

- University of Surrey FHMS Annual Festival of Research (poster) 


\title{
University of Surrey FHMS Annual Festival of Research 2009 - abstract
}

Title

Authors

Group

Abstract

\author{
THE EFFECTS OF TIME OF TREATMENT ON STATIN MUSCLE TOXICITY: \\ INITIAL CHARACTERIZATION OF A HUMAN MUSCLE CELL LINE \\ ROWENA GEE, NICK PLANT, JONATHAN JOHNSTON, KATE PLANT \\ Biochemical Sciences Division: Pharmacology \& Toxicology and Sleep \& Chronobiology \\ Themes
}

Statins are competitive inhibitors of 3-hydroxy-3-methylglutaryl coenzyme A (HMG$\mathrm{CoA}$ ) reductase, the rate-limiting enzyme in cholesterol biosynthesis. They are the first line of treatment for hypercholesterolemia, a known risk factor for the development of cardiovascular disease. Although statin therapy is generally considered to be safe, some patients experience muscle toxicity (myopathy), which is the most notable adverse effect of the statins and in the most severe cases can prove fatal. It is thought that myopathy most likely occurs due to the secondary effects of inhibiting HMG-CoA reductase, for example the effects on synthesis of other isoprenoids and the resulting disruption of protein prenylation.

Daily circadian rhythms are known to regulate a multitude of biological processes, and many aspects of cholesterol synthesis and metabolism have been shown to be under circadian control. To date, most studies looking into the optimum time for statin dosing focus on the effects of time on their cholesterol-lowering efficacy; however it is not currently known how temporal dosing of statins affects muscle toxicity. We propose that, like many aspects of cholesterol synthesis and metabolism, protein prenylation is also under circadian control. By looking into the effects of temporal dosing on cholesterol synthesis and protein prenylation, it may prove possible to achieve greater separation between the cholesterol-lowering effects of statins and unwanted toxicity. For this work we will use human liver and muscle cells in culture as a model system.

In the first phase of this project we have identified a human undifferentiated muscle cell line, the $\mathrm{RD}$ human thabdomyosarcoma cell line which has previously been used for studying the myotoxic effects of statins. We have studied differentiating $\mathrm{RD}$ cells to characterise the morphological and molecular changes which occur during this process and to confirm the expression of genes involved in cholesterol metabolism and circadian rhythmicity. Current work is employing real-time PCR to investigate temporal changes of gene expression. 
University of Surrey FHMS Annual Festival of Research 2009 - poster

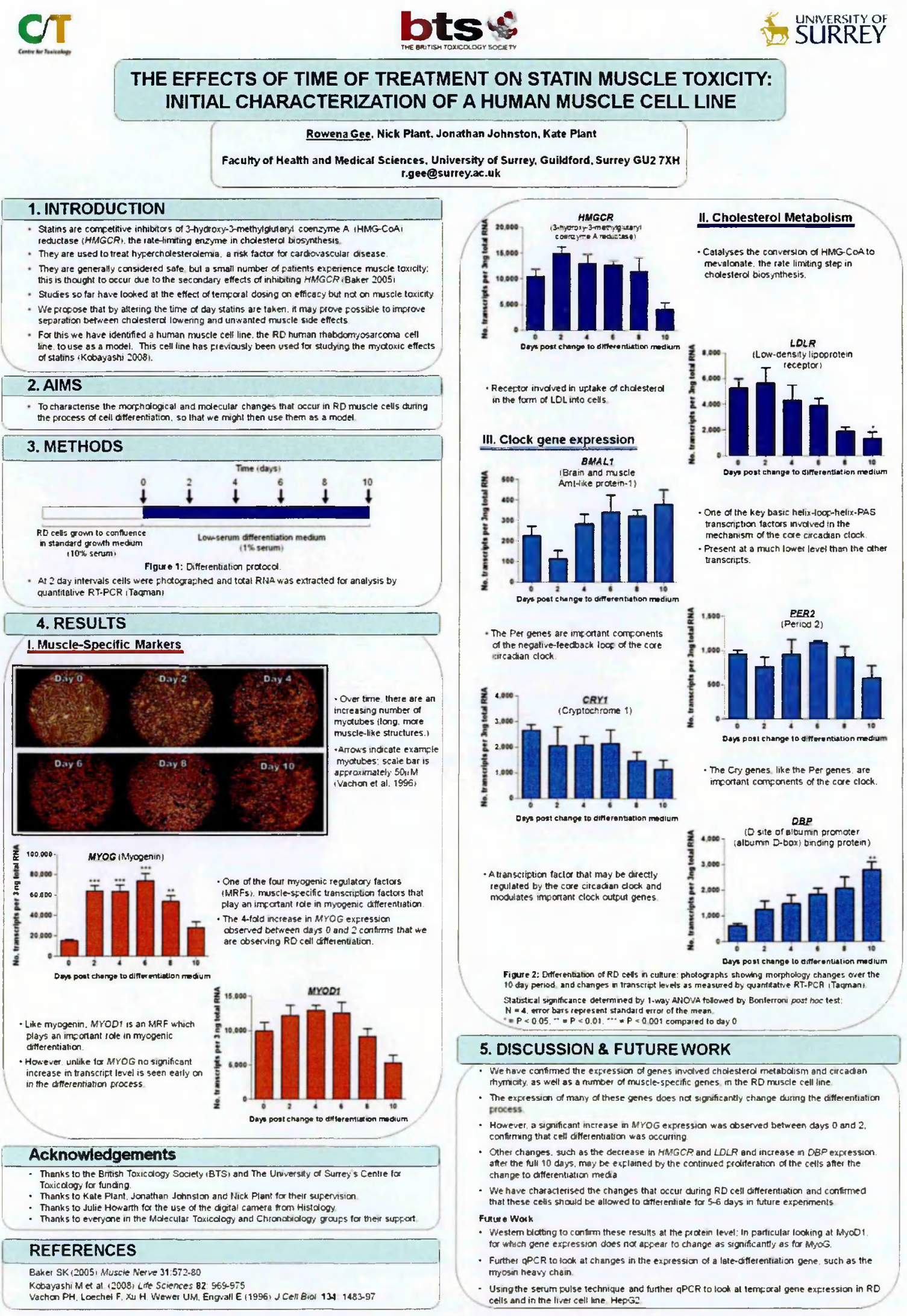




\title{
British Toxicoiogy Society (BTS) Annual Congress 2010 - abstract
}

\section{The Effects Of Time Of Treatment On Statin Muscle Toxicity: Preliminary Characterization Of A Human Muscle Cell Line}

\author{
$\underline{\text { Rowena H Gee }}^{1}$, Nick J Plant ${ }^{1}$, Jonathan D Johnston ${ }^{1}$, Kate E Plant ${ }^{1}$ \\ Centre for Toxicology, FHMS, University of Surrey, Guildford, Surrey GU2 7XH. r.gee@,surrey.ac.uk
}

Statins are competitive inhibitors of 3-hydroxy-3-methylglutaryl coenzyme A reductase (HMGCR), the rate-limiting enzyme in cholesterol biosynthesis, and are used to treat hypercholesterolemia, a risk factor for cardiovascular disease. They are generally considered safe, but a small number of patients experience muscle toxicity; which is thought to occur due to the secondary effects of inhibiting HMGCR, for example the disruption of protein prenylation (Baker 2005). Studies so far have looked at the effect of temporal dosing on efficacy but not on muscle toxicity. Since muscle is known to also show circadian changes in gene expression in mice (McCarthy et al. 2007), we hypothesise that by altering the time of day statins are taken, it may prove possible to improve separation between cholesterol lowering and unwanted muscle side effects.

The RD human rhabdomyosarcoma cell line has previously been used for studying the myotoxic effects of statins (Kobayashi et al. 2008). In the current work, we first characterised the morphological and molecular changes that occur during $\mathrm{RD}$ cell differentiation. We have also begun to characterise the gene expression changes that occur across circadian time, initially focussing on clock genes (Per2, BMALI and $C R Y I$ ) and $H M G C R$ and the LDL receptor. Preliminary statin dosing experiments were also carried out in order to determine the optimum statin dose for future experiments.

$\mathrm{RD}$ cells were grown to confluence in standard growth medium (DMEM containing $10 \%$ fetal bovine serum), before the initiation of differentiation by changing to low-serum medium (1\%). At 48 hour intervals cells were imaged and total RNA extracted for analysis by quantitative RT-PCR (Taqman). Statistical significance was determined by one-way ANOVA followed by Bonferroni post hoc test.

Over differentiation time an increased number of long myotubes was observed in culture. This was accompanied by an observed 4-fold increase in the expression of the muscle regulatory factor myogenin, which is consistent with the occurrence of cell differentiation; this peaked at day five before decreasing. The expression of key cholesterol metabolism-related and clock genes in the RD cell line was also examined, showing steady expression of $H M G C R$, the LDL receptor ( $L D L r)$, and clock genes PER2, BMAL1 and CRYI. In light of the results, it was decided to use a period of 5 days for RD cell differentiation prior to future experiments.

3-(4,5-dimethylthiazol-2-yl)2,5-diphenyl tetrazolium bromide (MTT) assays were also carried out, in order to assess the effect of simvastatin on cell viability. RD cells were seeded at a density of $2 \times 10^{3}$ cells per well on 96-well plates and left to differentiate for 5 days in low-serum medium. Various concentrations of simvastatin were then added, in a total of $100 \mu l$ serum-free medium per well. For the final 2.5 hours of the 48 hour dosing period $10 \mu$ l of PBS-containing MTT solution $(5 \mathrm{mg} / \mathrm{mL})$ was added to the medium. The MTT medium was then replaced with $100 \mu 1$ dimethylsulfoxide and the absorbance read at $540 \mathrm{~nm}$. Absorbance measured was expressed as a percentage of the control.

The observed IC50 value of simvastatin on the growth of differentiating RD muscle cells $(0.58 \mu \mathrm{M})$ 
was in a similar range to values seen in the literature (Kobayashi et al. 2008). However, further assays using a larger range of concentrations are needed to confirm this.

References: Baker, S.K., 2005 Muscle Nerve 31, 572-580

Kobayashi, M. et al. 2008 Life Sci. 82, 969-975

McCarthy, J.J. et al. 2007 Physiol Genomics 31, 86-95

Funding from The British Toxicology Society and The University of Surrey's Centre for Toxicology.

\section{University of Surrey FHMS Annual Festival of Research 2010 - abstract}

Title THE EFFECTS OF TIME OF TREATMENT ON STATIN MUSCLE TOXICITY: PRELIMINARY CHARACTERIZATION OF A HUMAN MUSCLE CELL LINE

Authors Rowena Gee, Nick Plant, Jonathan Johnston, Kate Plant

Group Biochemical Sciences Division: Drug Design, Cancer \& Toxicity and Sleep, Chronobiology \& Neurodisorders themes.

Abstract

Statins are competitive inhibitors of 3-hydroxy-3-methylglutaryl coenzyme A reductase (HMGCR), the rate-limiting enzyme in cholesterol biosynthesis, and are used to treat hypercholesterolemia, a known risk factor for cardiovascular disease. They are generally considered safe, but a small number of patients experience muscle toxicity; which is thought to occur due to the secondary effects of inhibiting HMGCR, for example the disruption of protein prenylation.

Daily circadian rhythms are known to regulate a multitude of biological processes, and many aspects of cholesterol synthesis and metabolism have been shown to be under circadian control. Studies so far have looked at the effect of temporal dosing on statin efficacy but not on muscle toxicity. Since muscle is known to show circadian changes in gene expression in mice, we hypothesise that by altering the time of day statins are taken, it may be possible to improve separation between cholesterol lowering and unwanted muscle side effects.

The RD human rhabdomyosarcoma cell line has previously been used for studying the myotoxic effects of statins. In the current work, we first characterised the morphological and molecular changes that occur during $\mathrm{RD}$ cell differentiation. We have also begun to characterise the gene expression changes that occur across circadian time, in both RD cells and the human hepatoma cell line Huh7, initially focusing on clock genes (PER2, BMAL1 and CRY1), HMGCR and $L D L R$. Preliminary statin dosing experiments were also carried out in order to determine the optimum statin dose for future experiments. 


\title{
Poster for BTS Annual Congress and FHMS Festival of Research 2010
}

\author{
btses
}

SURREY

\section{THE EFFECTS OF TIME OF TREATMENT ON STATIN MUSCLE TOXICITY: PRELIMINARY CHARACTERIZATION OF A HUMAN MUSCLE CELL LINE}

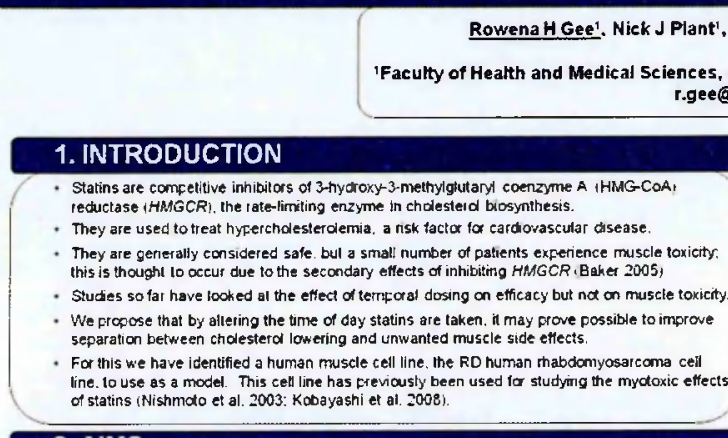

\section{AIMS}

To characterise the rmarchulogical and molecular changes thal occur in RD muscle cells clunng To carry out Freliminary statin dosing experiments. to determine the oftimesn dose for futur

\begin{tabular}{|c|c|}
\hline \multicolumn{2}{|l|}{ 3. METHODS } \\
\hline & 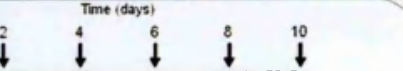 \\
\hline \multirow[t]{2}{*}{$\begin{array}{l}\text { RD cels grown lo confuence } \\
\text { in standard grosth medum } \\
110 \% \text { serum! }\end{array}$} & $\begin{array}{l}\text { Lowserum ditherentation medum } \\
\text { (16s serum) }\end{array}$ \\
\hline & Differentiation Fidocol. \\
\hline \multicolumn{2}{|c|}{$\begin{array}{l}\text { - At } 2 \text { day intervals cells were phodographed and todal RTIA was extracted for analysis by } \\
\text { quantitative RT-PCR (Taquan? }\end{array}$} \\
\hline \multicolumn{2}{|c|}{ 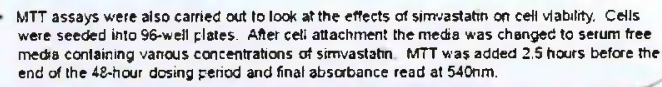 } \\
\hline
\end{tabular}

I) Characterization of Muscle Differentiation A. Muscle-Spectlic Markers

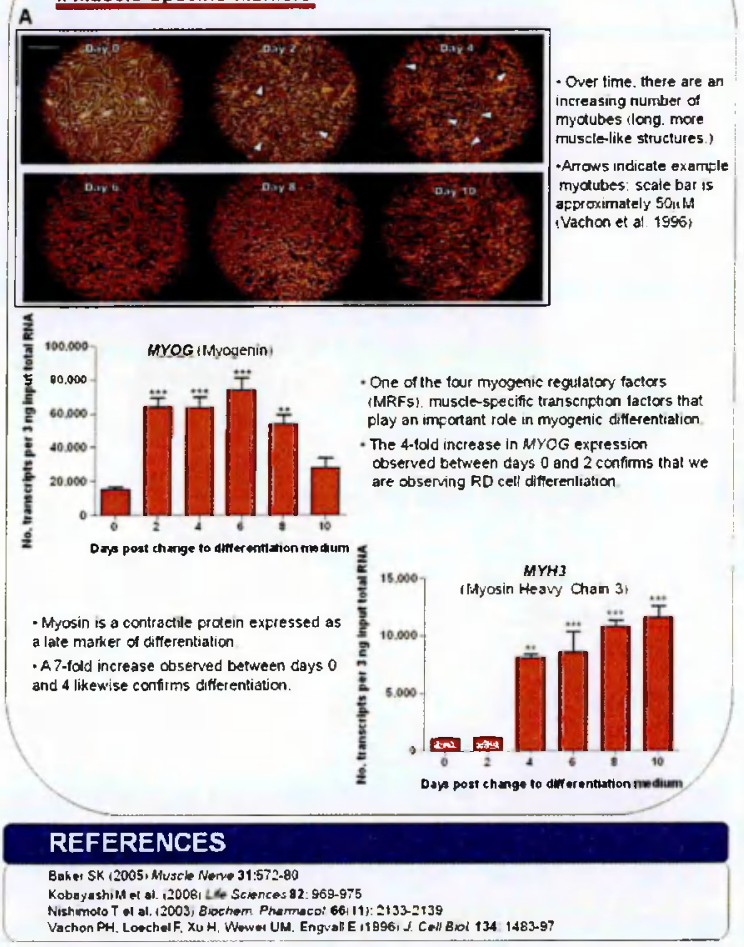

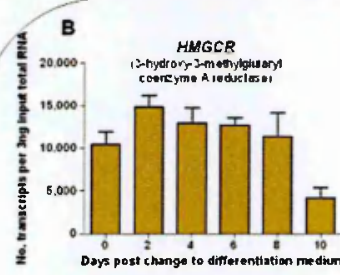

- Receptor involved in ustake of chotesterol in the form of LDL into cells.

iii. Clock Gene Expression

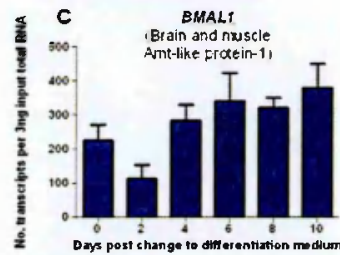

ii. Cholesterol Metabolism

Catalyses the conversion of HMG-COA to mevalonate, the rate limiting slep in

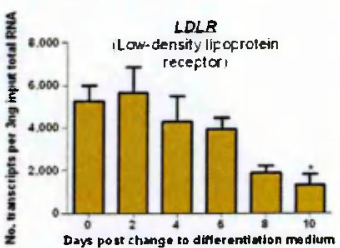

One of the key basic helix-look-helix.pas transcriction factors involved in the Present at a much lower ievel then the other Present at and
Iranserifts.

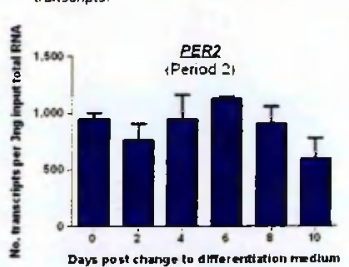

Figure Z: Differentiation of RD cells in culture: A) photographs showing morphotogy changes over

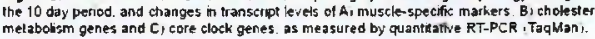
Statistical signific ance determined by l-way ANOVA followed by Bonterioni post hoc test $N=4$, eror bats iepresent standard error of the mean iSEN)

II) Characterization of Statin Toxicity

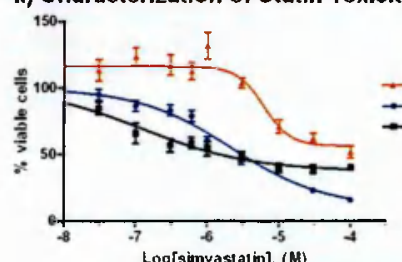

- HepG2 $\rightarrow R D-U$

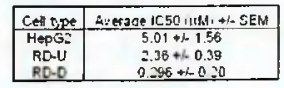
Log[simvastalim]. (Mit)

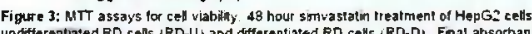
as a percentage of the control didelned as $100 \%$ i.

$\mathrm{N}=3$. etror bars represent standard enror of the mean i5EM)

\section{DISCUSSION \& FUTURE WORK}

We have confirmed the expression of genes involved cholesterd metabdism and cire adian

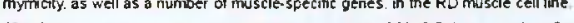

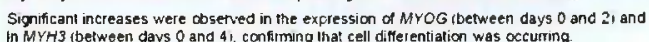
- encer Other changes. such as the decrease in HMGCR and LOLR affer the full 10 days. may be The expression of BMAL1 and PER2. genes involved in the core ciccadian clock. does not The expression of BMAL1 1 and PER? getes involved
significantly change during the differentiation Frocess

We have characlerised the changes that occur curing RD cell ditterentiation and based on in turure expenments.

Using MTT assays we heve also staned to chasacterise slatin toxicity in HepG: and RD cells. with differentiated $\mathrm{RO}$ cells showing greater senstitivity to simvastatin than either unditherentialed RO cells or HepG? cels.

Future Woik

- Western blatting to confirm these results al the prodein level.

- Finigh characle izalion of statin toxicity in RD and HepG? cells.

Using the serum pulse lechnique and further APCR to look al temcaral gene expression in RD 


\title{
British Toxicology Society (BTS) Annual Congress 2011 - abstract
}

\author{
Differential Sensitivity to Toxicity of Statins: A Comparison Of Liver And Muscle Cell Lines
}

\author{
Rowena H Gee ${ }^{1}$, Nick J Plant ${ }^{1}$, Jonathan D Johnston ${ }^{1}$, Kate E Plant ${ }^{1}$ \\ Centre for Toxicology, FHMS, University of Surrey, Guildford, Surrey GU2 7XH. \\ r.gee@surrey.ac.uk
}

Statins are competitive inhibitors of 3-hydroxy-3-methylglutaryl coenzyme A reductase (HMGCR), the rate-limiting enzyme in cholesterol biosynthesis. They are widely used to treat hypercholesterolemia, a risk factor for cardiovascular disease. Whereas they are generally considered safe, muscular toxicity is a common side effect, thought to occur due to the secondary effects of inhibiting HMGCR, such as disruption of protein prenylation (Baker 2005).

Whereas previous studies have investigated the effect of statins on cholesterol metabolism in liver cells, few studies have investigated this effect in skeletal muscle, the major site of toxicity (Mullen 2010). Through the use of two human cell lines we have undertaken a comparison of the effects of a variety of statins on cholesterol metabolism in liver and muscle. We hypothesise that differences observed between these cell lines may help to further elucidate the mechanism for statin-induced muscle toxicity. First, the RD human rhabdomyosarcoma cell line was chosen for its skeletal muscle phenotype, and its previous use for studying the myotoxic effects of statins (Kobayashi et al. 2008); second, the $\mathrm{HuH} 7$ hepatocellular carcinoma cell line was used to represent human liver parenchyma.

Initially, 3-(4,5-dimethylthiazol-2-yl)2,5-diphenyl tetrazolium bromide (MTT) assays were carried out, assessing metabolic and cellular viability. $\mathrm{RD}_{\text {diff }}$ cells were seeded on 96 -well plates and left to differentiate for 5 days in low-serum medium, while Huh7 and $\mathrm{RD}_{\text {undiff }}$ cells were seeded 24 hours prior to experiment. Cells were exposed to various statin concentrations for 48 hours in serum-free medium, with MTT-containing PBS added for the final 2.5 hours. Media was replaced with DMSO and MTT absorbance read at $540 \mathrm{~nm}$. Absorbance was expressed as a percentage of the control, and values plotted as mean \pm SEM of three independent experiments, where $\mathrm{N}=6$ in each experiment. Statistical significance of differences in observed IC50 values determined by unpaired t-test.

We observed statistically significant reductions in cell viability for all three cell types, Huh7; $\mathrm{RD}_{\text {undiff }}$ and $\mathrm{RD}_{\text {diff, }}$, on treatment with simvastatin $(\mathrm{Na})$, simvastatin (lactone), lovastatin $(\mathrm{Na})$, lovastatin (lactone), cerivastatin, fluvastatin, atorvastatin, rosuvastatin and pravastatin in a dosedependent manner. The largest reductions in cell viability were observed with the most lipophilic statins: simvastatin $(\mathrm{Na})$, lovastatin $(\mathrm{Na})$ and cerivastatin, whereas significant loss of cell viability was only observed with very high concentrations of the hydrophilic pravastatin $(1000 \mu \mathrm{M}$ and greater).

Higher cell toxicity was observed for all statins in the RD cells, either differentiated or undifferentiated, compared to the Huh7 cells. All observed IC50 values, that we have been able to determine, appear to be within a similar range of values as seen in the literature (Kobayashi et al. 2008). As one example, IC50 values in response to fluvastatin treatment were $8.51 \pm 5.49 \mu \mathrm{M}$ (Huh7), $6.02 \pm 1.83 \mu \mathrm{M}$ ( $\left.R D_{\text {undiff }}\right)$ and $2.62 \pm 2.56 \mu \mathrm{M}\left(\mathrm{RD}_{\text {diff }}\right)$.

Taken together, these data represent the first comprehensive comparative analysis of statin toxicity in human liver and muscle cell lines. Such characterisation is an important validation step in the 
characterisation of differences in the response of these cell lines to statins, and hence the elucidation of the full mechanism of toxicity.

References: Baker, S.K., 2005 Muscle Nerve 31, 572-580

Kobayashi, M. et al. 2008 Life Sci. 82, 969-975

Mullen, P. et al 2010 Biochem. Pharmacol 79, 1200-1209

Funding from The British Toxicology Society and The University of Surrey's Centre for Toxicology.

\section{University of Surrey FHMS Annual Festival of Research 2011 - abstract}

\section{DIFFERENTIAL SENSITIVITY TO TOXICITY OF STATINS: A COMPARISON OF LIVER AND MUSCLE CELL LINES}

R.H. Gee, N.J. Plant, J.D. Johnston, K.E. Plant

Drug Design, Cancer \& Toxicity; Biochemical Sciences Division

Statins are competitive inhibitors of 3-hydroxy-3-methylglutaryl coenzyme A reductase (HMGCR), the ratelimiting enzyme in cholesterol biosynthesis. They are widely used to treat hypercholesterolemia, a risk factor for cardiovascular disease. Whereas they are generally considered safe, muscular toxicity is a common side effect, thought to occur due to the secondary effects of inhibiting HMGCR, such as disruption of protein prenylation.

Previous studies have investigated the effect of statins on cholesterol metabolism in liver cells, however few studies have investigated this effect in skeletal muscle, the major site of toxicity. Through the use of two human cell lines we have undertaken a comparison of the effects of a variety of statins on cholesterol metabolism in liver and muscle. We hypothesise that differences observed between these cell lines may help to further elucidate the mechanism for statin-induced muscle toxicity. The RD human rhabdomyosarcoma cell line was chosen for its skeletal muscle phenotype, and its previous use for studying the myotoxic effects of statins; the Huh7 hepatocellular carcinoma cell line was used to represent human liver.

Initially, 3-(4,5-dimethylthiazol-2-yl)2,5-diphenyl tetrazolium bromide (MTT) assays were carried out, assessing metabolic and cellular viability. We observed dose-dependent reductions in cell viability for all three cell types used, Huh7, $\mathrm{RD}_{\text {undiff }}$ and $\mathrm{RD}_{\text {diff, }}$ on treatment with simvastatin $(\mathrm{Na})$, simvastatin (lactone), lovastatin (Na), lovastatin (lactone), cerivastatin, fluvastatin, atorvastatin, rosuvastatin and pravastatin. The largest reductions in cell viability were observed with the most lipophilic statins: simvastatin (Na), lovastatin (Na) and cerivastatin, whereas significant loss of cell viability was only observed with very high concentrations of the hydrophilic pravastatin (1000microM and greater). Higher cell toxicity was observed for all statins in the RD cells, either differentiated or undifferentiated, compared to the Huh7 cells. Taken together, these data represent the first comprehensive comparative analysis of statin toxicity in human liver and muscle cell lines. 


\section{Poster for BTS Annual Congress and FHMS Festival of Research 2011}

\section{CT}

\section{bts 4}

\section{DIFFERENTIAL SENSITIVITY TO TOXICITY OF STATINS: A COMPARISON OF LIVER AND MUSCLE CELL LINES}

Rowena H Gee. Nick J Plant. Jonathan D Johnston and Kate E Plant

Faculty of Health and Medical Sciences, University of Surrey, Guildford. Surrey GU2 7XM r.gee@surrey.ac.uk

\section{INTRODUCTION}

Statins are competive inhibiors of 3-hydoxy-3-methygoutany coenzyme A IHMG-COA -

They are used to treat hyperchdesterdemia, a nsk factor for cardowa scular asease.

They pre gener ally considered sate. but a small number d patients exferience muscle loxichlych

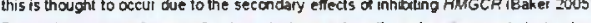

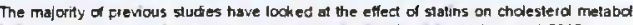
Through the use of two human cell lines we have undertaken a comparison of the eflects of a variety or stains an cholesterd melabdism in both her and muste. For his work we have model this cett line has a sheleal muscle chenotyse and has kreviously been used for chosen the Huhi human hefatocellular caranoma cell hine as oul camparison liver cell tine. tor statin-tinduced muscie toxicity.

\section{METHODS}

- Rith assays were cartied out to assess the eflects of a variety of statins on cell viabality

- The effect of statin uearment on toxal cellular cholesterd was measuled to ex amine statin-

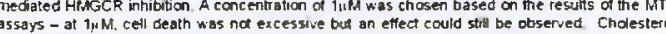
was quantrifed using the Amclex Red Chdesterd Assay kit. See figure 1 lor ivither details.

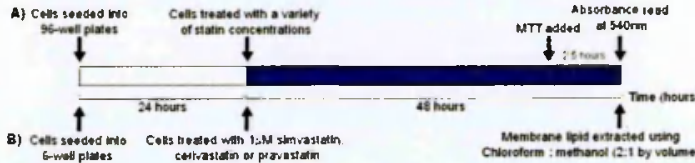

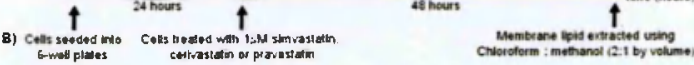

Figure 9: Expermental sesign for $A_{i}$ UTT assays and Bi chotersemoi assays

\section{RESULTS}

I) Characterization of Statin-Mediated Cell Toxicity

Simvastatin and lovastatin are administered an maelive frodugs llacecone formi which are then convented to the active oxen actd forms in the booy. These are ine most lifachilic of

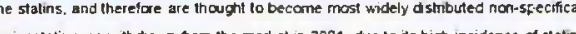
induced muscle toxichty

A A

Rosury stalin and pravastalun are hydroxhilic. and theretore the least likely 10 become distributed non-speerfically
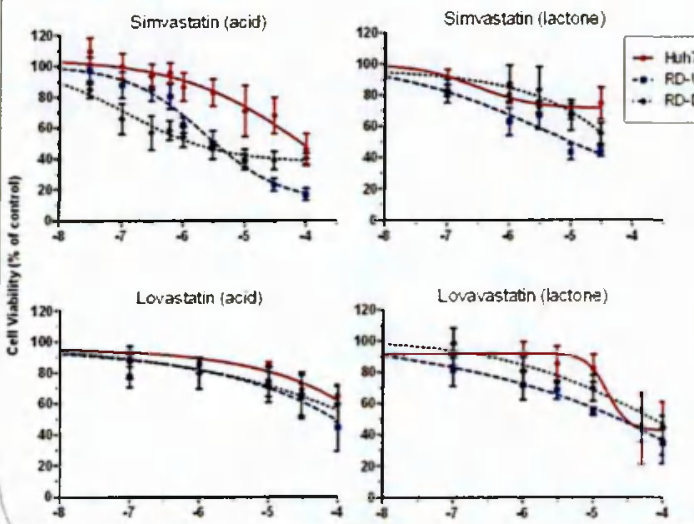

Log (stminin). (M)

\section{REFERENCES}

Eaker SK 12005, Mussch Neno 31.572.80

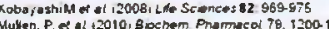

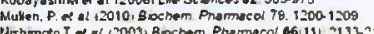
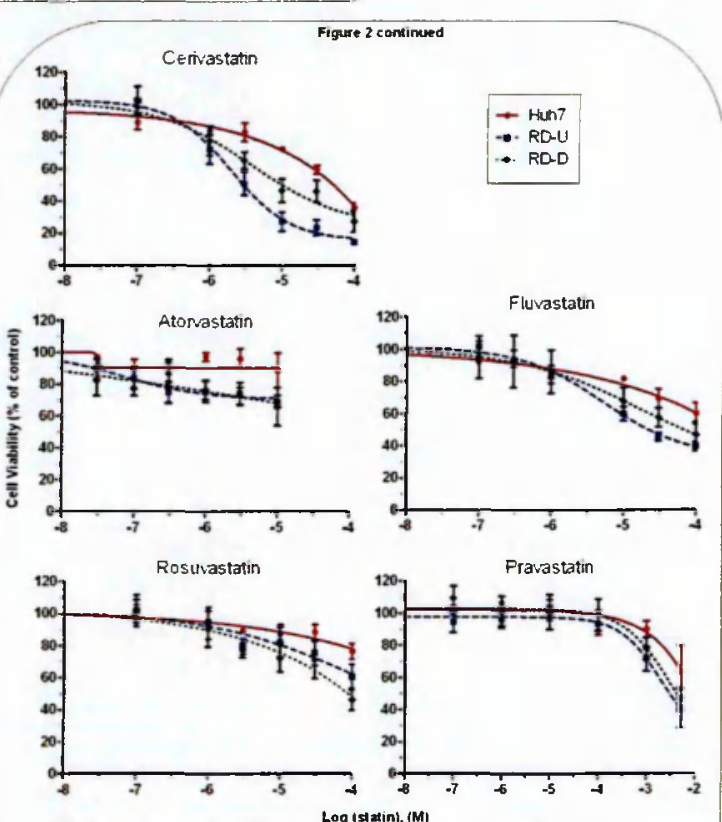

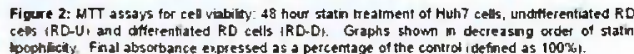

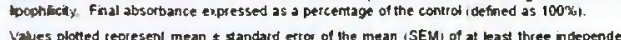
baurs plonted represenl mean 4 sandard ent

II) Effect of Statins on Total Cellular Cholesterol

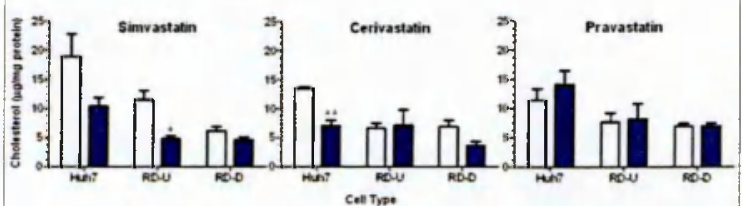

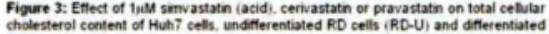
cholesterol content of Huht cells. unditterentiated RD cells (RD-U) and ditterentiated

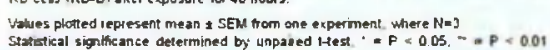
Contral

\section{DISCUSSION \& FUTUREWORK}

This is the first comprehensive corticarative snalysis of statin toxichly in liver and muscle cetl lines ingure 21

We observed a reduction in cell viablity in all three cell lypes, on tie alment with all statins. in

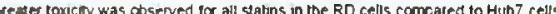

The largest reductions in cell viability were coserved on treatment with the most lipophilic (lestin cacidi, lovastatin and cerivastatin.

Sugnificant loss $\alpha$ cell hablity was only observed with very high concentrations $\alpha$ the

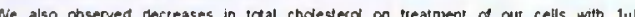

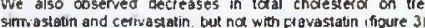

Pulue Work

Heasure toxal cellular choless erol ater exposure 10 higher concentrations of simvastatin facid

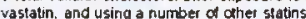

iteasure dher cellular eflects in resfonse 10 slatin exposure, inclucting reduction in prodein

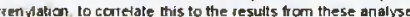




\section{British Toxicology Society (BTS) Annual Congress 2012 - abstract for oral presentation}

Differential effects of statins on protein prenylation in liver and muscle cell lines

Statins inhibit 3-hydroxy-3-methylglutaryl coenzyme A reductase (HMGCR), the rate-limiting enzyme in cholesterol biosynthesis, and are widely used to treat hypercholesterolemia, a risk factor for cardiovascular disease. Muscular toxicity is a side effect, thought to occur due to the secondary effects of inhibiting HMGCR, such as disruption of protein prenylation. Through the use of two human cell lines we have undertaken a comparison of the effects of statins on cell viability and protein prenylation in liver and muscle. We anticipate that differences observed may help to further elucidate the mechanism for statin-induced muscle toxicity.

Total protein extracted from cells treated with a range of simvastatin and cerivastatin concentrations was subjected to western blot analysis using the geranylgeranylated protein Rap1A as a marker of prenylation.

In both cell lines unprenylated Rap1A was observed following statin treatment; this was dose-dependent for both statins. Both cell lines were more sensitive to cerivastatin than simvastatin with a 10 -fold difference in dose required to produce detectable levels of unprenylated Rap1A. On statin treatment, disruption of Rap1A prenylation occurred at a 10-fold lower concentration in muscle than in liver cells, both for cerivastatin $(1 \mu \mathrm{M}$ and $10 \mu \mathrm{M}$ respectively) and simvastatin.

This data adds to our previous toxicity data, which showed that RD muscle cells were significantly more sensitive to both of these statins than Huh7 liver cells. This supports a mechanism whereby disruption in the intermediates in the cholesterol synthesis pathway, including the isoprenoids used in protein prenylation, is the primary cause of statin-induced muscle toxicity. 


\begin{abstract}
Joint $19^{\text {th }}$ International Symposium on Microsomes and Drug Oxidations (MDO) and $12^{\text {th }}$ European meeting of the International Society for the Study of Xenobiotics (ISSX) 2012 - abstract
\end{abstract}

\title{
The Muscle Cell Line RD Is More Sensitive To Statin-Mediated Disruption Of Protein Prenylation Than The Liver Cell Line Huh7: Implications for Statin- Mediated Toxicity
}

\author{
Rowena H. Gee, Nick J. Plant, Jonathan D. Johnston and Kate E. Plant, \\ Department of Biochemistry and Physiology, University of Surrey, Guildford, United \\ Kingdom
}

Statins inhibit 3-hydroxy-3-methylglutaryl coenzyme A reductase (HMGCR), the rate-limiting enzyme in cholesterol biosynthesis, and are widely used to treat hypercholesterolemia, a risk factor for cardiovascular disease. A common adverse effect associated with statin use is muscular aches, which can rarely progress to the potentially fatal rhabdomyolysis. The mechanism underlying this toxicity is still not fully understood, but secondary effects of HMGCR inhibition, such as disruption of protein prenylation, have been suggested as a likely mechanism. We have compared the effects of statins on both cell viability and protein prenylation in human liver and skeletal muscle cell lines, hypothesising that statins would preferentially disrupt prenylation within the muscle cells. Initially, 3-(4,5dimethylthiazol-2-yl)2,5-diphenyl tetrazolium bromide (MTT) assays were carried out, in order to assess metabolic and cellular viability after cells were exposed to different statin concentrations. We observed significant reductions in cell viability for both cell lines, on treatment with simvastatin, cerivastatin and pravastatin in a dose-dependent manner. The largest reductions in cell viability were observed with the most lipophilic statins, simvastatin and cerivastatin, whereas significant loss of cell viability was only observed with very high concentrations of the hydrophilic pravastatin $(1000 \mu \mathrm{M}$ and greater). Higher cell toxicity was observed for all statins in RD muscle cells compared to Huh7 liver cells. Subsequently, western blot analysis was used to examine the prenylation status of the geranylgeranylated protein Rap1A in total protein extracts from cells treated with a range of simvastatin and cerivastatin concentrations. Unprenylated Rap1A was observed in both cell lines, in a dose-dependent manner, following treatment with simvastatin and cerivastatin. This prenylation deficiency was a specific effect resulting from statin inhibition of HMGCR since co-treatment with mevalonate, a downstream intermediate in the metabolic pathway, rescued the phenotype. Both cell lines were more sensitive to cerivastatin than simvastatin, with a 10-fold difference in dose required to produce detectable levels of unprenylated Rap1A. In addition, statin treatment resulted in disruption of Rap1A prenylation at a 10-fold lower concentration in muscle than in liver cells, both for simvastatin $(1 \mu \mathrm{M}$ and $10 \mu \mathrm{M}$ respectively) and cerivastatin $(0.1 \mu \mathrm{M}$ and $1 \mu \mathrm{M}$ respectively). Together these data confirm that RD muscle cells, which are significantly more sensitive to statin treatment, do show preferential disruption of protein prenylation when compared to liver cells. This supports a mechanism whereby disruption in the intermediates in the cholesterol synthesis pathway, including the isoprenoids used in protein prenylation, is the primary cause of statin-induced muscle toxicity. 


\section{University of Surrey FHMS Annual Festival of Research 2012 - abstract DIFFERENTIAL EFFECTS OF STATINS ON PROTEIN PRENYLATION IN LIVER AND MUSCLE CELL LINES}

R.H. Gee, N.J. Plant, J.D. Johnston, K.E. Plant

Drug Design, Cancer \& Toxicity; Department of Biochemistry and Physiology

Statins inhibit 3-hydroxy-3-methylglutaryl coenzyme A reductase (HMGCR), the rate-limiting enzyme in cholesterol biosynthesis, and are widely used to treat hypercholesterolemia, a risk factor for cardiovascular disease. Muscular toxicity is a side effect, thought to occur due to the secondary effects of inhibiting HMGCR, such as disruption of protein prenylation. We have compared the effects of statins on both cell viability and protein prenylation in human liver and skeletal muscle cell lines, hypothesising that statins would preferentially disrupt prenylation within the muscle cells.

Total protein extracted from cells treated with a range of simvastatin and cerivastatin concentrations was subjected to western blot analysis using the geranylgeranylated protein Rap1A as a marker of prenylation.

In both cell lines unprenylated Rap1A was observed following statin treatment; this was dose-dependent for both statins. Both cell lines were more sensitive to cerivastatin than simvastatin with a 10 -fold difference in dose required to produce detectable levels of unprenylated Rap1A. On statin treatment, disruption of Rap1A prenylation occurred at a 10-fold lower concentration in muscle than in liver cells, both for simvastatin (1 $\mu \mathrm{M}$ and $10 \mu \mathrm{M}$ respectively) and cerivastatin $(0.1 \mu \mathrm{M}$ and $1 \mu \mathrm{M}$ respectively).

These data add to our previous toxicity data, which showed that RD muscle cells were significantly more sensitive to both of these statins than Huh7 liver cells, and confirm that RD muscle cells do show preferential disruption of protein prenylation when compared to liver cells. This supports a mechanism whereby disruption in the intermediates in the cholesterol synthesis pathway, including the isoprenoids used in protein prenylation, is the primary cause of statin-induced muscle toxicity. 


\section{The Muscle Cell Line RD Is More Sensitive To Statin-Mediated Disruption Of Protein Prenylation Than The Liver Cell Line Huh7: Implications For Statin-Mediated Toxicity}

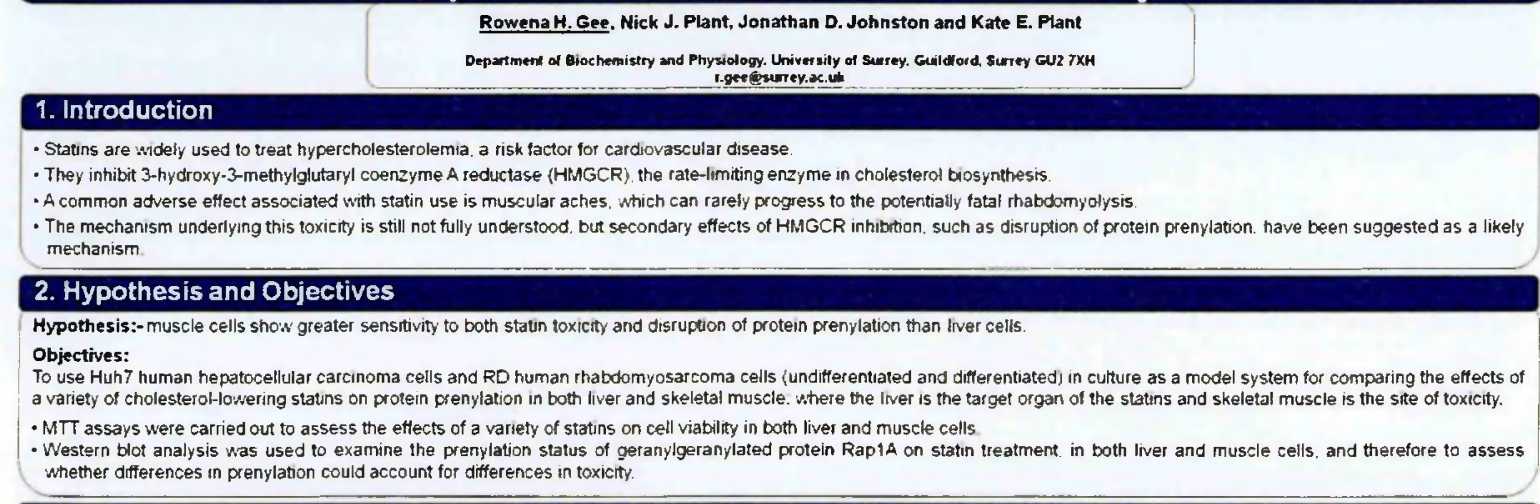

\section{Results}

\section{l) Characterization Or Statin-Mediated Cell Toxicity}

- Simvastatin is one of the most lipophilic statins and is thought to become distributed to a wide variety of tissues via passive diffusion. Simvastatin also remains one of the most a mde variety of tissues via passive diff
frequently prescribed statins in the UK

- Cervastatin was withdrawn from the worldside market in 2001 . due 10 its high incidence of statim-induced muscle toxicity

- Pravastatin is hydrophilic and therefore the least likely to become sidely distributed Pravastatin has also shown much lower levels of muscle toxicty in patients in the chinic

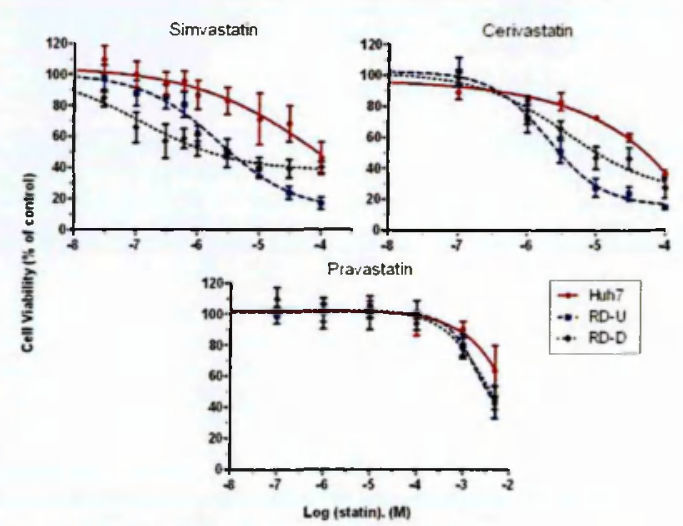

Flour 1 1: Cell viability in response to statin treatmert

Huhi cells. unditherentiated RD cells IRD-U, and difterentiated RD cets IRD-D, wer trested with stahn for t8 hours and if

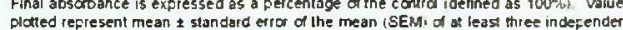
expenments, where 6 reglicates wele used in each experiment

\section{II) Effects of Statins On Prenylation}

- Rap1A is a smatl GTPase that undergoes geranylger anylation

- Its prenylation status can be determined using a combination of antibodies RapiA - detects the unprenylated protein Beta-actin - used as a loading control

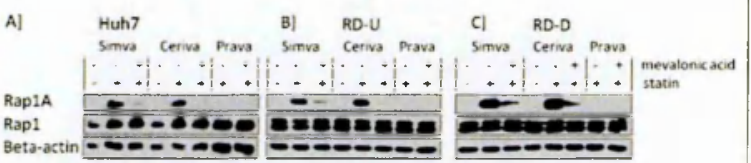

Figure 2: Entoct of stadin treatment on Rap1 A prenylation

Total Frodein was extracted trom Huh7. RD-U and RD-D cells treated for 48 hours wilh vehicl control statin. or mevalonk acid in actikon to statin. Simvagsabn and cravastatin were used - concentring a ta 100yul Western bits are restesentative od three independent experiments

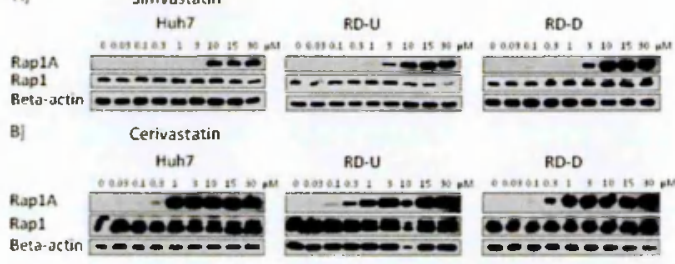

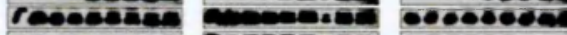

Figus 3: Dose-dependent iffects of statin tre ament on RapiA prenylation Taal brotein was extracted forn cells treated to 43 hours with vehicle control or a sange of

\section{Conclusions}

ii Statin treatment leads to differential cell toxiciti

- Statin treatment led to a reduction in cell viability, in all cell types, in a dose-dependent manner (Figure 1)

- The largest reductions in cell viability were observed on treatment with lipophilic simvastatin and cerivastatin. with loss of cell viability observed only with very high concentrations of hydrophilic pravastatin (1000) $1 \mathrm{M}$ and greater)

- Greater toxicity was observed for all statins in RD muscle cells compared to Huh7 Iiver cells: this difference was statistically signficant with simvastatin and cerivastatin treatment ( $P<0.0001$ two-way ANOVA). This is consistent with the observation of skeletal muscle toxicity as a well knosn adverse effect of the statins in the clinic

ii) Statin treatment leads to differential discuption of protein prenylation

Simvastatn and cerivastatin treatment led to detection of unprenylated RapiA (Figure 2)

This was not due to a change in levels of total Rapl protein and co-treatment with mevalonate rescued this change in prenylation status

- Muscle and liver cells were both more sensitive to cerivastatn than simvastatin (Figure 3).

- Lower concentrations of both simvastatin and cerivastatin were needed to distupt protein prenylation in muscle compared to liver cells.

Together these data confirm that RD muscle cells. which are significantly more sensitive to statin treatment. do show preferential disruption of prote in prenylation when compared to liver cells. This supports a mechanism whereby dissuption in the intermediates in the cholesterol synthesis pathway, including the Isoprenoids used in
ptotein prenylation, is the primary cause of statintinduced muscle toxicity. 